\title{
Application of ecient Monte Carlo photon beam simulations to dose calculations in voxellized human phantoms
}

Citation for published version (APA):

Walters, B. R. (2017). Application of ecient Monte Carlo photon beam simulations to dose calculations in voxellized human phantoms. [Doctoral Thesis, Maastricht University]. Datawyse / Universitaire Pers Maastricht. https://doi.org/10.26481/dis.20171214bw

Document status and date:

Published: 01/01/2017

DOI:

10.26481/dis.20171214bw

Document Version:

Publisher's PDF, also known as Version of record

\section{Please check the document version of this publication:}

- A submitted manuscript is the version of the article upon submission and before peer-review. There can be important differences between the submitted version and the official published version of record.

People interested in the research are advised to contact the author for the final version of the publication, or visit the DOI to the publisher's website.

- The final author version and the galley proof are versions of the publication after peer review.

- The final published version features the final layout of the paper including the volume, issue and page numbers.

Link to publication

\footnotetext{
General rights rights.

- You may freely distribute the URL identifying the publication in the public portal. please follow below link for the End User Agreement:

www.umlib.nl/taverne-license

Take down policy

If you believe that this document breaches copyright please contact us at:

repository@maastrichtuniversity.nl

providing details and we will investigate your claim.
}

Copyright and moral rights for the publications made accessible in the public portal are retained by the authors and/or other copyright owners and it is a condition of accessing publications that users recognise and abide by the legal requirements associated with these

- Users may download and print one copy of any publication from the public portal for the purpose of private study or research.

- You may not further distribute the material or use it for any profit-making activity or commercial gain

If the publication is distributed under the terms of Article $25 \mathrm{fa}$ of the Dutch Copyright Act, indicated by the "Taverne" license above, 


\section{Application of efficient Monte Carlo photon beam simulations to dose calculations in voxellized human phantoms}

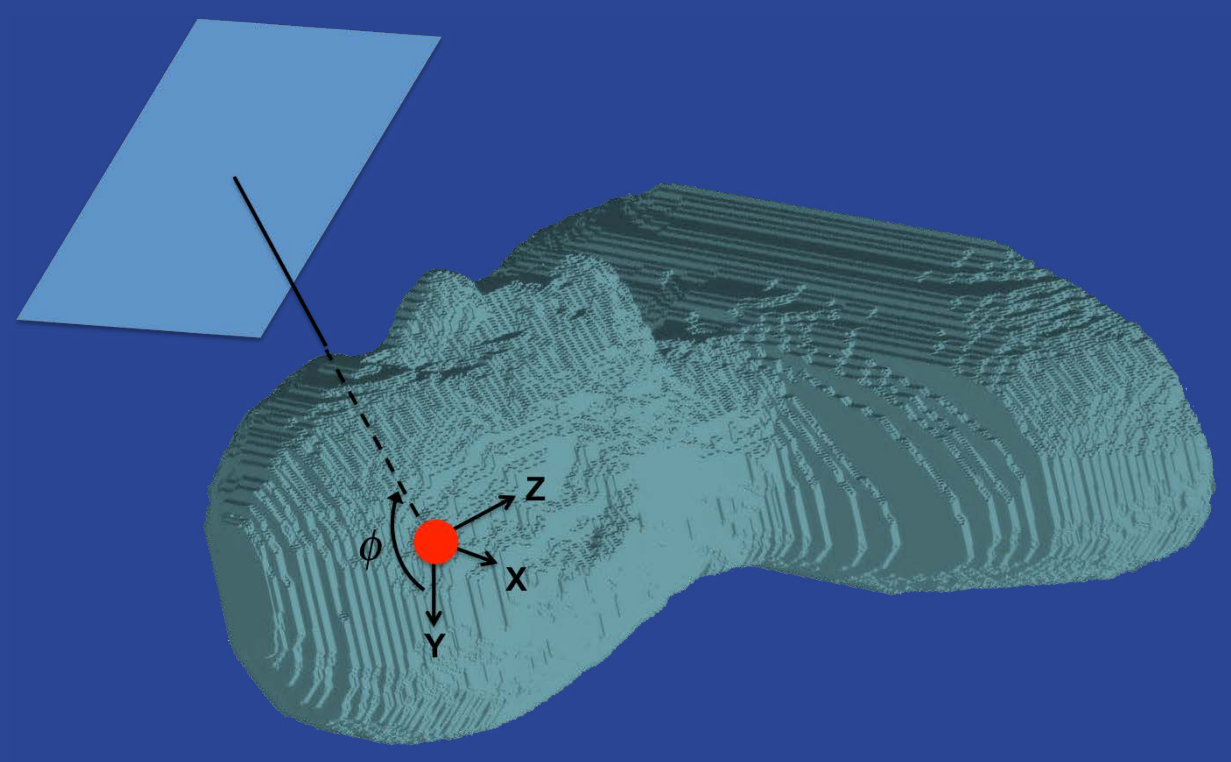

Blake Walters 
Cover image modified from B.R.B. Walters, G.X. Ding, R. Kramer, I. Kawrakow, "Skeletal dosimetry in cone beam computed tomography," Med Phys 36:2915-2922, 2009.

ISBN 9789461597823

Print Datawyse | Universitaire Pers Maastricht

\section{Copyrights}

\section{(C) 2017 B.R.B. Walters}

All rights reserved. No part of the material protected by this copyright notice may be reproduced or utilised in any form or by any means, electronic or mechanical, including photocopying, recording or by any information storage and retrieval system, without written permission from the author and the publisher(s) holding the copyrights of the reprinted article(s). 


\title{
Application of efficient Monte Carlo photon beam simulations to dose calculations in voxellized human phantoms
}

\author{
DISSERTATION
}

to obtain the degree of Doctor at

Maastricht University on the authority of the Rector Magnificus

Prof. dr. Rianne M. Letschert

in accordance with the decision of the Board of Deans,

to be defended in public

on Thursday $14^{\text {th }}$ of December 2017 at 14:00 hours

by

Blake Reginald Walters

Born on November $3^{\text {rd }} 1966$

Toronto (Canada) 


\section{Supervisors:}

Prof. F. Verhaegen

Prof. D.W.O. Rogers (Carleton University, Ottawa, Ontario, Canada)

\section{Assessment Committee:}

Prof. R.L. Westra (Chair)

Dr. W.J.C. van Elmpt

Prof. B.W. Raaymakers, University Medical Centre Utrecht, The Netherlands

Prof. J. Seco, Heidelberg University, Heidelberg, Germany

\section{Funded by:}

Ionizing Radiation Standards, Measurement and Science Standards, National Research Council of Canada Bldg M-35, 1200 Montreal Road

Ottawa, Ontario, Canada K1A 0R6 
I General introduction and methods 1

II History by history statistical estimators in the BEAM code system 13

III A “HOWFARLESS" option to increase efficiency of homogeneous phantom calculations with DOSXYZnrc 31

IV Large efficiency improvements in BEAMnrc using directional bremsstrahlung splitting

V Increasing efficiency of BEAMnrc-simulated Co-60 beams using directional source biasing

VI Skeletal dosimetry in cone beam computed tomography

VII Dose to medium vs dose to water as an estimator of dose to sensitive skeletal tissue

VIII Discussion and Summary

Summary

Acknowledgements

List of Publications 179

Curriculum Vitae 181

Valorization 

General introduction and methods 



\subsection{Introduction}

For over two decades, Monte Carlo (MC) simulations have been perceived as the gold standard for calculating dose for radiotherapy treatment planning (RTP) and radiation protection applications. This is primarily due to the ability of MC to handle complex geometries that are either intractable using analytical methods or else require a sufficient number of simplifying assumptions to make the uncertainty on the resultant dose calculation clinically significant. For example, MC can accurately model transport across the interface between media (tissues) with different atomic numbers ( $Z$ ), while the pencil beam algorithms traditionally used in radiotherapy treatment planning can result in dose inaccuracies on the order of $10 \%$ or greater [1, 2]. In addition, MC simulations can be used to provide snapshots of the treatment beam over the course of its interactions with accelerator and patient (and other structures), with implications for treatment design and optimization. Analytical methods simply cannot provide this information.

Research in the therapeutic application of MC simulations of radiation transport dates back to the 1960's when Berger published a description of the MC treatment of charged particle transport that would ultimately become the backbone of the ETRAN MC code[3]. One of the historic challenges in applying MC calculations to radiation transport is the treatment of charged particles, which interact continuously via elastic and inelastic collisions over the course of their trajectory. In order to simulate this transport in a reasonable time, electron transport codes must make use of multiple scattering theories, such as those by Molière[4] and Goudsmit and Saunderson[5], which, in turn, may require certain approximations. Each charged particle step in the simulation then encompasses multiple interactions and is, thus, known as a condensed history $(\mathrm{CH})$ step. By contrast, photon and neutron transport, both of which feature relatively long mean free paths between well-defined interactions were already relatively well-understood, and MC simulations had been in use for some time to simulate photons and neutron interactions in nuclear reactors.

Berger[3] identified two classes of MC simulation codes based on their treatment of electron transport: Class I codes include all charged particle interactions in each $\mathrm{CH}$ step, while class II codes include a subset of interactions in each $\mathrm{CH}$ step, simulating the remaining interactions explicitly. This implies that class II codes are potentially more accurate than Class I codes depending on a judicious choice of which interactions to model in detail. The aforementioned ETRAN code is an example of a Class I code, while the well-benchmarked MC code, EGS4[6], and its modern evolution, containing improved physics, EGSnrc[7], are class II codes. In the case of EGS4/EGSnrc, the selection criteria for explicit simulation of charged particle interactions are the energy of secondary particles and the type of interaction: particle energy must be above a cutoff energy, AE, below which cross section data are not tabulated, and then only annihilation events, Bhabha and Moller scattering and bremsstrahlung events are simulated explicitly.

There are a number of other class II codes which have found extensive application in radiotherapy and radiation protection research, including MCNP[8], PENELOPE[9] and Geant4[10]. Similar to EGS4/EGSnrc, these codes have achieved acceptance throughout the medical physics research community owing to extensive benchmarking and their ability to match experimental data where available. 
However, EGS4/EGSnrc remains the most widely used MC simulation platform among researchers and it is with applications based on the physics in EGS4/EGSnrc that the research described in this thesis is concerned.

User applications based on EGS4/EGSnrc developed over the history of the code include DOSXYZnrc[11], for calculating dose in a phantom comprising rectilinear voxels, BEAMnrc[12, 13], optimized for the simulation of radiotherapy treatment heads (see Figure 1.1), along with a number of codes[14] for calculating dose, fluence and stopping power ratios in cylindrical phantoms. More recently, a flexible object-oriented geometry package, egspp[15] has been developed.

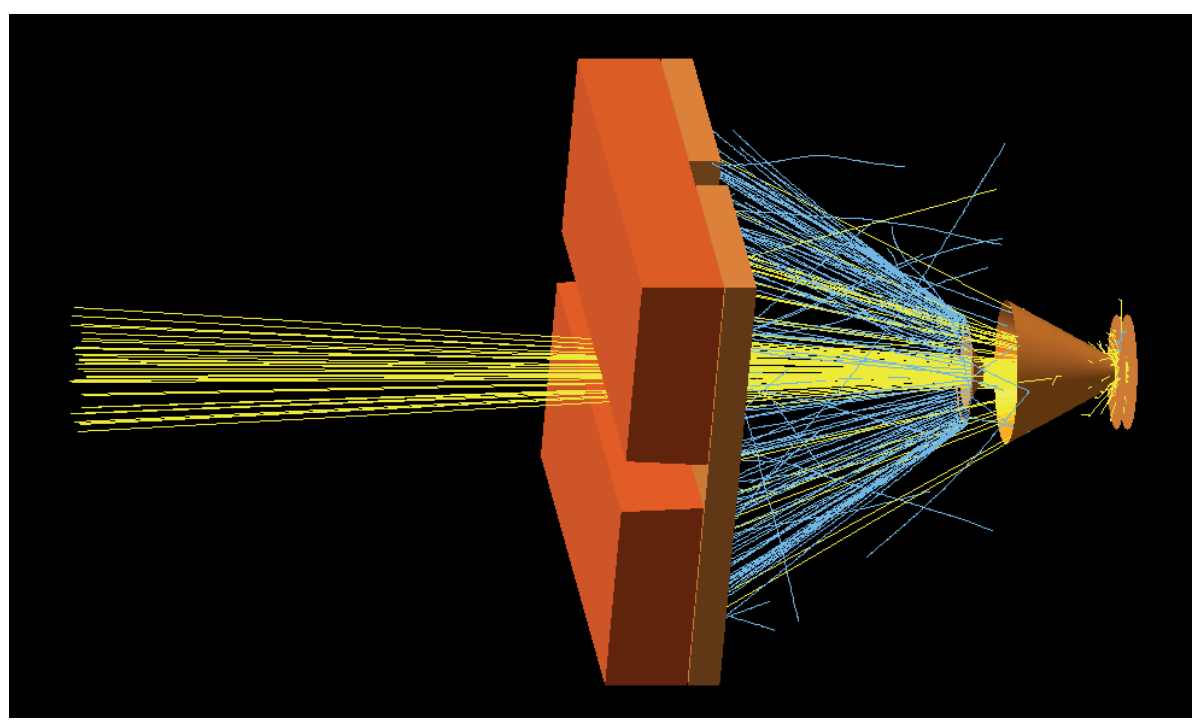

Figure 1.1: Schematic of a BEAMnrc simulation of $6 \mathrm{MV}$ photon beam $\left(10 \times 10 \mathrm{~cm}^{2}\right.$ field $)$ from a Siemens KD2 accelerator. Photon tracks are shown in yellow, while contaminant electron tracks are blue. Directional bremsstrahlung splitting (DBS) with electron splitting is used to preferentially generate photons directed into the field and simultaneously increase the number of contaminant electrons. The main structural components of the accelerator are, from left: field defining jaws, flattening filter, primary collimator, target. Accelerators are simulated by concatenating geometry modules (component modules) optimized for simulating components typically used in linear accelerators.

One of the main obstacles to the regular use of MC simulations in clinical radiotherapy treatment planning (RTP) has historically been the lack of affordable computing power, specifically CPU speed. Until recently, MC dose calculations to the desired precision could take days to weeks of CPU time. This situation has been partially ameliorated by running calculations in parallel (e.g., EGSnrc has a built-in parallel processing functionality requiring minimal effort to adapt to the user's system). However, the applicability of such large-scale, multi-core simulations remains in the realm of research.

In obeisance of Moore's Law, however, CPU performance continues to increase exponentially, and the last decade has seen computing speeds available even on relatively cheap desktop PCs that would seem 
to put regular, clinical MC RTP within grasp. Indeed, several manufacturers of commercially-available treatment planning systems (TPSs) now incorporate MC simulations into some portion of their dose calculations. These include Varian Eclipse $e^{\circ}$ Raystation ${ }^{\circ}$, and Elekta $\mathrm{XiO}^{\circ}$.

Moreover, the decreasing cost of data storage means that it is now practical to store large phase space files from simulated treatment heads, such as those generated by BEAMnrc, for the purpose of downstream dose calculations.

In addition to increased CPU speed, an essential component of the effort to make routine MC simulation a reality in clinical radiotherapy has been the development of techniques to increase the efficiency of MC codes. This has been particularly true of the ongoing development of the EGSnrc system and its associated applications and is another reason for the continued popularity of this system.

Techniques to increase the efficiency of EGSnrc codes fall into two general categories: 1) CPU timesaving techniques and 2) variance reduction techniques. CPU time-saving techniques involve algorithms for decreasing the time required to transport particles, usually at the cost of some acceptable decrease in accuracy, while variance reduction techniques reduce the uncertainty in calculated quantities while preserving the underlying transport physics.

An example of a CPU time-saving technique implemented early on in EGS4/EGSnrc is charged particle range rejection. Here, the transport of charged particles is ceased, and their energy deposited on the spot, if they are unable to make it to the nearest geometric region boundary with an energy greater than some user-defined cutoff (ECUT). While this scheme can increase the efficiency of simulations by a factor of 2, judicious selection of the energy below which the particle range is evaluated (ESAVE) is required to prevent a significant impact on the low-energy bremsstrahlung radiation simulated[12].

Variance reduction techniques in EGSnrc codes have typically fallen under two broad categories: interaction splitting and interaction forcing. Both of these techniques decrease the uncertainty inherent in sampling probability functions by sampling them many times per event. The result is the production of correlated particles. However, if these particles (and their products) are scored downstream in different zones/voxels, then correlations are lost and the uncertainty in each statistically-independent event (primary history) can decrease significantly.

Variance reduction techniques have been employed in BEAMnrc almost since its introduction, with early examples including photon interaction forcing and uniform bremsstrahlung splitting (UBS). Selective splitting of bremsstrahlung events into a user-defined field (SBS) was introduced by Sheikh-Bagheri in 1999[16] and increased the efficiency of photon beam dose calculations by factors of up to 10 .

The technique of splitting radiative events can also be applied to isotropically radiating sources (e.g., Cs-137 and Co-60 capsules). Efficient simulation of these sources is of current interest as image-guided radiotherapy (IGRT) devices using Co-60 treatment heads in tandem with magnetic resonance imaging (MRI) emerge on the market. In addition, efficient simulation of Co-60 beams makes routine MC calculation of beam correction factors a feasible prospect.

While fast and accurate simulation of treatment beams is essential to any MC dose calculation, the ultimate goal is an accurate estimate of the dose delivered. As mentioned above, several commercially available TPSs use MC calculations to calculate dose for electron or photon beams. The details of how the 
phase space data for incident treatment beams is constructed are not known for all systems and likely vary from system to system. Apart from this, however, calculation of dose in CT-based phantoms presents its own challenges. Some of these will be addressed in more detail in the Discussion at the end of this thesis.

In addition to the patient-specific dose calculations mentioned above, by using voxellized phantoms representing an "average" human, MC simulations are uniquely able to determine the dose to radiosensitive components of bone, such as bone marrow and bone surface cells. The field of MC treatment planning is currently in the midst of a decade-and-a-half long debate[17-20] over whether dose to medium, $\mathrm{D}_{m}$, or dose to water-in-medium, $\mathrm{D}_{w}$, is a more useful specifier of dose. This debate is fraught with issues that are historic, philosophical, and rooted in a lack of available data. However, it is acknowledged that the difference is only important in bone. Therefore, a realistic estimate of dose to sensitive tissue within bone is capable of providing information which will help guide the choice of quantity to specify.

\subsection{Methods}

This section is intended to give a brief, general overview of the methods covered in much more detail in each chapter.

\subsubsection{EGSnrc}

As indicated above, all MC simulations presented in this work use the EGSnrc code and associated applications. EGSnrc simulates photon, electron and positron transport, benchmarked over an energy range of $1 \mathrm{keV}$ to several hundred $\mathrm{GeV}$. With respect to charged particle transport, EGSnrc introduced a number of enhancements over EGS4 that have a significant impact on accuracy. Among these:

- Condensed history $(\mathrm{CH})$ transport uses an exact solution to the muliple scattering angular distribution based on the theories of Goudsmit and Saunderson[5] and Kawrakow and Bielajew[21, 22].

- Spin effects can be incorporated in the elastic scattering cross section.

- Electron impact ionization can be simulated using one of five possible cross section data sets.

- The user has the option to cross geometric boundaries in single scattering mode, eliminating any inaccuracies inherent in crossing in multiple scattering mode (i.e., using the PRESTA boundary crossing algorithm).

Since its introduction in 2000, there have been further improvements in EGSnrc, including:

- The option to simulate atomic relaxation processes in shells $\mathrm{K}$ through $\langle\mathrm{N}\rangle$. This includes the production of fluorescent photons, Auger electrons and Coster-Kronig electrons.

- The option to simulate triplet production.

- The option to include radiative Compton scattering corrections.

- The option to include photonuclear interactions. 
Even with the above-mentioned enhancements there is much room for ongoing development and refinement of the existing physics in EGSnrc. For example, the effects of electron impact ionization are not well understood, nor has the algorithm been completely benchmarked, and atomic relaxations processes are currently confined to shells with binding energies $>1 \mathrm{keV}$. Another important limitation of EGSnrc is its inability to simulate protons and heavy ions which, given current trends in radiotherapy, may have serious implications for the code's general utility in the future. This will be discussed in more detail in chapter VIII.

\subsubsection{Efficiency}

Efficiency with respect to the MC calculations described here is given as:

$$
\epsilon=\frac{1}{s^{2} T}
$$

where $s$ is an estimate of the uncertainty on the quantity of interest (e.g., dose or fluence) and $T$ is the CPU time.

Calculating meaningful values of efficiency depends on having a good estimate of the uncertainty, $s$. Earlier versions of EGS4/EGSnrc codes estimated $s$ by dividing up calculations into statistical batches. In the early 2000's, however, this method was replaced by a history by history estimate in which each primary history is treated as a statistically independent event and correlations between particles are accounted for. The latter consideration is especially important given that efficient photon beam simulations sometimes require splitting interactions, resulting in thousands of correlated particles per primary history. In addition, the history by history method results in a significant decrease in fluctuations in the estimate of uncertainty (i.e., the "variance on the variance").

\subsubsection{Interaction splitting}

Interaction splitting is essential to several variance reduction schemes implemented in EGSnrc codes. Very briefly, it involves splitting an interaction (usually bremsstrahlung) a user specified number of times, $N_{\text {split }}$, with the resultant particles each having their statistical weight reduced by a factor of $1 / N_{\text {split }}$. Energies and directions for the resultant split particles are then sampled from known distributions. Provided that these split particles and their descendents are not all scored in the same region of interest, this multiple sampling results in a significant decrease in the variance of an MC calculation.

Figure 1.2 shows a simplified schematic of the directional bremsstrahlung splitting (DBS) routine covered in more detail in chapter IV.

In the case of split bremsstrahlung interactions, secondary charged particles are generally subject to russian roulette with a survival probability $1 / N_{\text {split }}$ (with survivors having their weight increased by a factor $N_{s p l i t}$ ). This prevents excessive CPU time from being consumed following charged particle secondaries set in motion by the split photons. Should the user be interested in contaminant electron dose, DBS includes a method to recover these charged particles. 


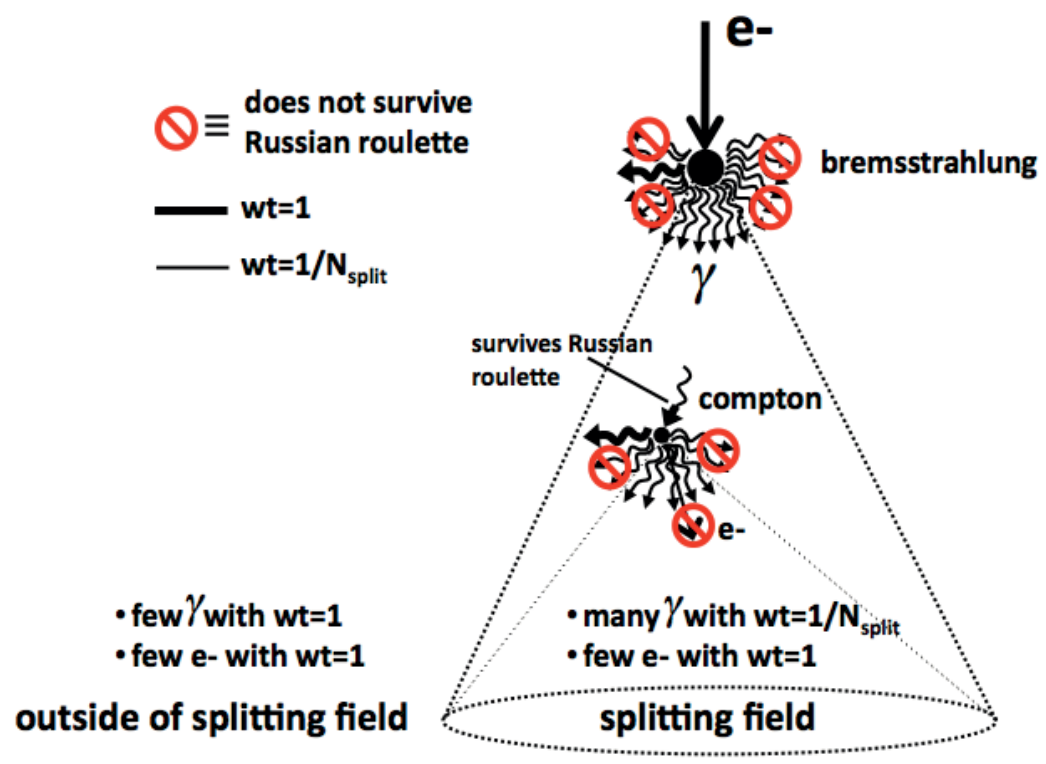

Figure 1.2: Schematic of the directional bremsstrahlung splitting (DBS) routine. Bremsstrahlung events are split $N_{\text {split }}$ times. Photons directed into a user-defined, circular splitting field are kept and have their weight reduced by a factor of $1 / N_{\text {split }}$ (i.e., they are "thin"), while photons directed outside this field are subject to Russian roulette with survival probability $1 / N_{\text {split }}$. Surviving photons retain weight 1 (i.e., they are "fat") and represent the physics for these particles. Secondary electrons created by thin photons are also subject to Russian roulette with survival probability $1 / N_{\text {split }}$, as are any scattered photons directed outside the splitting field. Efficiency is further improved by subjecting thin photons about to undergo a scattering event (Compton, photoelectric or pair production) to Russian roulette with survival probability $1 / N_{\text {split }}$. If the photon survives, it becomes fat and the scattering event is split $N_{\text {split }}$ times, with the resultant split photons treated in the same manner as those resulting from a bremsstrahlung event. The end result of DBS is that many photons, all with low weight, will be directed into the field of interest, few photons, all with high weight, will be directed outside it, and there will be few charged particles, all with high weight, everywhere.

\subsubsection{Voxellized human phantoms (FAX/MAX)}

The final two papers in this thesis present dose calculations in a set of voxellized human phantoms. These phantoms, representing an adult female (FAX) and male (MAX), were constructed by Kramer et al[23] based on patient CT images. Their most recent versions (FAX06/MAX06) were specifically coded to perform dose calculations using the radiation transport physics in EGSnrc for radiation safety applications and feature organs with compositions agreeing with those recommended by ICRP[24] and ICRU[25]

Microstructure within bone spongiosa (red bone marrow and trabecular bone) in FAX06/ MAX06 is simulated during particle transport by overlaying spongiosa voxels with a repeating pattern 
of microvoxels (with dimensions on the order of $10 \mu \mathrm{m}^{3}$ ) containing either trabecular bone or red bone marrow to create a micromatrix. The structure of the micromatrix, itself, is based on actual $\mu \mathrm{CT}$ image data.

\subsection{Contents of this thesis}

The research described herein can be grouped into two broad themes: variance reduction in EGSnrc MC simulations of photon beams and the application of photon beam simulations to dose calculations in voxellized human phantoms.

The first paper[26], presented in chapter II, describes in detail the history by history method for estimating uncertainties implemented in all EGSnrc applications starting in 2000. Comparisons are made between the history by history method and the prior method of statistical batches with respect to the variance on the uncertainty estimate and the ability to account for correlations between particles. Specific attention is given to phase space sources, which often contain a limited amount of data and in which particles, thus, must be reused in order to obtain acceptable statistics in downstream calculations.

Chapter III presents a paper[27] published on a CPU time-saving technique, called HOWFARLESS, for increasing the efficiency of dose computation in homogeneous phantoms simulated using the EGSnrc application, DOSXYZnrc. The HOWFARLESS technique saves significant CPU time by disregarding internal voxel boundaries when determing the maximum distance covered by a charged particle during a condensed history step. The details of the HOWFARLESS algorithm are presented, and the accuracy of the method relative to standard transport stopping at internal voxel boundaries (i.e ., using HOWFAR) is analyzed.

Chapter IV is a paper[28] describing the directional bremsstrahlung splitting (DBS) algorithm, which, since its implementation in 2004, has become a standard technique for efficient BEAMnrc simulations of photon beams. A description of the algorithm is given, including the electron splitting option to improve statistics on dose from contaminant electrons. The paper gives a detailed analysis of efficiency for fluence and dose calculations, comparing the performance of DBS to selective bremsstrahlung splitting (previously the most efficient bremsstrahlung splitting routine) and uniform bremsstrahlung splitting, and makes recommendations for the optimum settings of splitting number, splitting field size and electron splitting parameters.

A paper[29] describing a directional splitting technique for efficiently simulating treatment heads that use isotropically radiating sources, called directional source biasing (DSB), comprises chapter V. DSB was recently implemented in BEAMnrc and has been shown to increase the efficiency of Co-60 dose calculations by a factor of up to 400 , making MC commissioning of Co- 60 beams feasible. The DSB algorithm is described, any potential systematic biases introduced are analyzed, and a detailed study of efficiency, including recommended splitting numbers, is presented.

The paper[30] in chapter VI presents dose calculations for a simulated cone beam CT (CBCT) scan of the voxellized human phantoms, FAX06 and MAX06, for the purpose of determining additional dose to organs and to the radiosensitive tissue in trabecular bone-red bone marrow and bone surface cells- 
when this imaging modality is used for IGRT. Dose is calculated for scans at three different sites-head and neck, chest, pelvis-each characterized by bone having a different trabecular bone volume fraction (TBVF).

Chapter VII is a paper[31] describing research that makes further use of FAX06 and MAX06 to calculate the dose from megavoltage photon beams in red bone marrow and bone surface cells. These values are then compared to the dose calculated in bulk trabecular bone, or, dose to medium, $\mathrm{D}_{m}$, and the dose to water in medium, $\mathrm{D}_{w}$ at sites with different TBVFs. The results are used to deduce whether $\mathrm{D}_{m}$ or $\mathrm{D}_{w}$ provides a better estimate of dose to radiosensitive tissue in bone in an effort to introduce some computational rigour into the $\mathrm{D}_{m}$ vs $\mathrm{D}_{w}$ debate.

The Discussion in chapter VIII deals in detail with some of the important current and future implications of the research in the preceding chapters. The chapter includes new work addressing the issue of $\mathrm{D}_{m}$ to $\mathrm{D}_{w}$ conversion for kilovoltage beams using Burlin cavity theory and presents some thoughts about the current status of $\mathrm{MC}$ in commercially available treatment planning systems.

Summary remarks and future work are also presented in chapter VIII.

\subsection{Bibliography}

[1] J. Cygler, J. J. Battista, J. W. Scrimger, E. Mah, and J. Antolak. Electron dose distributions in experimental phantoms: a comparison with 2D pencil beam calculations. Phys. Med. Biol., 32:1073 1086, 1987.

[2] M. S. Huq, A. G. Agostinelli, and R. Nath. An evaluation of the recommendations of the TG25 protocol for determination of depth-dose curves for electron beams using ionization chambers. Med. Phys., 22:1333 - 1337, 1995.

[3] M. J. Berger. Monte Carlo Calculation of the penetration and diffusion of fast charged particles. In B. Alder, S. Fernbach, and M. Rotenberg, editors, Methods in Comput. Phys., volume 1, pages 135 215. Academic, New York, 1963.

[4] H. A. Bethe. Molière's theory of multiple scattering. Phys. Rev., 89:1256 - 1266, 1953.

[5] S. A. Goudsmit and J. L. Saunderson. Multiple scattering of electrons. Phys. Rev., 57:24 - 29, 1940.

[6] W. R. Nelson, H. Hirayama, and D. W. O. Rogers. The EGS4 Code System. Report SLAC-265, Stanford Linear Accelerator Center, Stanford, California, 1985.

[7] I. Kawrakow, E. Mainegra-Hing, D. W. O. Rogers, F. Tessier, and B. R. B. Walters. The EGSnrc Code System: Monte Carlo simulation of electron and photon transport. Technical Report PIRS-701 (5th printing), National Research Council of Canada, Ottawa, Canada, 2009.

[8] J. Briesmeister. MCNP-A general purpose Monte Carlo code for neutron and photon transport, Version 3A. Los Alamos National Laboratory Report LA-7396-M (Los Alamos, NM), 1986. 
[9] J. Sempau, E. Acosta, J. Baro, J. M. Fernandez-Varea, and F. Salvat. An algorithm for Monte Carlo simulation of coupled electron-photon transport. Nucl. Inst. Meth. B, 132, 1997.

[10] S. Agostinelli et al. GEANT4 - a simulation toolkit. Nucl. Inst. Meth. A, 506:250 - 303, 2003.

[11] B. R. B. Walters, I. Kawrakow, and D. W. O. Rogers. DOSXYZnrc Users Manual. NRC Report PIRS $794(\mathrm{rev} \mathrm{B}), 2005$.

[12] D. W. O. Rogers, B. A. Faddegon, G. X. Ding, C.-M. Ma, J. Wei, and T. R. Mackie. BEAM: A Monte Carlo code to simulate radiotherapy treatment units. Med. Phys., 22:503 - 524, 1995.

[13] D. W. O. Rogers, B. Walters, and I. Kawrakow. BEAMnrc Users Manual. NRC Report PIRS 509(a)revI, 2005.

[14] D. W. O. Rogers, I. Kawrakow, J. P. Seuntjens, and B. R. B. Walters. NRC User Codes for EGSnrc. Technical Report PIRS-702, National Research Council of Canada, Ottawa, Canada, 2000.

[15] I. Kawrakow. egspp: the EGSnrc C++ class library. Technical Report PIRS-899, National Research Council of Canada, Ottawa, Canada, 2005.

[16] D. Sheikh-Bagheri, I. Kawrakow, B. Walters, and D. W. O. Rogers. Monte Carlo simulations: Efficiency improvement techniques and statistical considerations. Integrating New Technologies into the Clinic: Monte Carlo and Image-Guided Radiation Therapy, Proceedings of the 2006 AAPM Summer School (Medical Physics, Madison, WI, 2006), pages 71-91.

[17] H. H. Liu and P. Keall. Point/Counterpoint: $\mathrm{D}_{m}$ rather than $\mathrm{D}_{w}$ should be used in Monte Carlo treatment planning. Med. Phys., 29:922 - 924, 2002.

[18] I. J. Chetty et al. Report of the AAPM Task Group No. 105: Issues associated with clinical implementation of Monte Carlo-based treatment planning. Med. Phys., 34:4818-4853, 2007.

[19] A. Carlsson Tedgren and G. Alm Carlsson. Specification of absorbed dose to water using modelbased dose calculation algorithms for treatment planning in brachytherapy. Phys. Med. Biol., 58: $2561-2579,2013$.

[20] P. Andreo. Dose to fhwater-likefh media or dose to tissue in MV photons radiotherapy treatment planning: still a matter of debate. Phys. Med. Biol., 60:309 - 337, 2015.

[21] I. Kawrakow and A. F. Bielajew. On the representation of electron multiple elastic-scattering distributions for Monte Carlo calculations. Nuclear Instruments and Methods, 134B:325 - 336, 1998.

[22] I. Kawrakow and A. F. Bielajew. On the condensed history technique for electron transport. Nuclear Instruments and Methods, 142B:253 - 280, 1998.

[23] R Kramer, H J Khoury, J W Vieira, and V J M Lima. MAX06 and FAX06: update of two adult human phantoms for radiation protection dosimetry. Phys. Med. Biol., 51:3331-3346, 2006. 
[24] ICRP. Basic Anatomical and Physiological Data for Use in Radiological Protection: Reference Values. Annals of the ICRP 89, ICRP, Washington D.C., 2002.

[25] ICRU. Tissue Substitutes in Radiation Dosimetry and Measurements. ICRU Report 44, ICRU, Washington D.C., 1989.

[26] B. R. B. Walters, I. Kawrakow, and D. W. O. Rogers. History by history statistical estimators in the BEAM code system. Med. Phys., 29:2745 - 2752, 2002.

[27] B. R. B. Walters and I. Kawrakow. A "HOWFARLESS" option to increase efficiency of homogeneous phantom calculations with DOSXYZnrc. Med. Phys., 34:3794 - 3807, 2007.

[28] Iwan Kawrakow, D. W. O Rogers, and B.R.B. Walters. Large efficiency improvements in BEAMnrc using directional bremsstrahlung splitting. Med. Phys., 31:2883 - 2898, 2004.

[29] B. R. B. Walters. Increasing efficiency of BEAMnrc-simulated Co-60 beams using directional source biasing. Med. Phys., 42:5817 - 5827, 2015.

[30] B. R. B. Walters, G. X. Ding, R. Kramer, and I. Kawrakow. Skeletal dosimetry in cone beam computed tomography. Med. Phys., 36:2915 - 2922, 2009.

[31] B. R. B. Walters, R. Kramer, and I. Kawrakow. Dose to medium versus dose to water as an estimator of dose to sensitive skeletal tissue. Phys. Med. Biol., 55:4535 - 4546, 2010. 
History by history statistical estimators in the BEAM code system 


\begin{abstract}
A history by history method for estimating uncertainties has been implemented in the BEAMnrc and DOSXYZnrc codes replacing the method of statistical batches. This method groups scored quantities (e.g. dose) by primary history. When phase-space sources are used, this method groups incident particles according to the primary histories that generated them. This necessitated adding markers negative energy! to phase-space files to indicate the first particle generated by a new primary history. The new method greatly reduces the uncertainty in the uncertainty estimate. The new method eliminates one dimension which kept the results for each batch! from all scoring arrays, resulting in memory requirement being decreased by a factor of 2 . Correlations between particles in phasespace sources are taken into account. The only correlations with any significant impact on uncertainty are those introduced by particle recycling. Failure to account for these correlations can result in a significant underestimate of the uncertainty. The previous method of accounting for correlations due to recycling by placing all recycled particles in the same batch did work. Neither the new method nor the batch method take into account correlations between incident particles when a phase-space source is restarted so one must avoid restarts.
\end{abstract}




\subsection{Introduction \& Theory}

\subsubsection{The batch approach to uncertainties}

The BEAM code system[1] is a widely used Monte Carlo code for simulating radiotherapy beams and calculating dose distributions in patients (see ref 2 for a listing of over 150 publications). We have upgraded the BEAM code system to create BEAMnrc [3, 4], which uses the recently released EGSnrc Monte Carlo code for radiation transport $[5,6]$. At the same time we have improved the method for estimating uncertainty in dose and fluence calculated in BEAMnrc and DOSXYZnrc. Previously, calculation of uncertainty in all quantities depended on splitting calculations into statistical batches (usually 10) and then, once the simulation was finished, taking the estimate of the uncertainty in the average of a scored quantity, $\mathrm{X}$, to be:

$$
s_{\bar{X}}=\sqrt{\frac{\sum_{i=1}^{N}\left(X_{i}-\bar{X}\right)^{2}}{N(N-1)}}
$$

where $\mathrm{N}$ is the number of batches, $X_{i}$ is the value of $\mathrm{X}$ in batch $\mathrm{i}$ and $\bar{X}$ is the mean value of $\mathrm{X}$ evaluated over all batches.

There are three problems with the batch approach. First, unless a large number of statistical batches is used, there are significant fluctuations in the uncertainty itself since the sample size, $\mathrm{N}$, in equation (1) is quite small. Second, arbitrarily grouping histories into batches ignores any correlations between incident particles. Incident particles will be correlated when phase space data from a BEAMnrc simulation of an accelerator is used as a source, especially if variance reduction techniques, such as bremsstrahlung splitting and photon forcing, are used in the accelerator simulation. Finally, the implementation of the batch approach used in most NRC user-codes added an extra dimension (storing the results from each batch) to all arrays scoring quantities of interest. This last limitation was especially evident in DOSXYZnrc, where the scoring arrays are already large because of the large number of geometrical regions (e.g., 2 million voxels for $128 \times 128 \times 128$ resolution). It should be noted however that this is a reflection of how this was coded in DOSXYZnrc and could have been avoided using other coding techniques.

\subsubsection{The history by history method}

In order to eliminate the problems with estimating uncertainty using batches, we have adopted a clever trick (attributed[7] to F. Salvat of the University of Barcelona) for efficiently implementing the history by history method for estimating uncertainty. It has been described by Sempau et al. [8]. The history by history method is well known and has been used for years in other codes (e.g., since at least 1986 in MCNP[9]). Andreo [10] has also pointed out its advantages. However, the brute force application of this method is inefficient and the inherent improvements did not justify the increase in computation time required to update all scored quantities after each history, especially in calculations with many scoring bins. Use of Salvat's approach removes this increase in computation time. We also found it necessary to make modifications to account for correlations between incident particles when a phase space source is 
used. Similar modifications were suggested independently by Sempau et al. [8].

Returning to Equation (1), let $X_{i}$ be the quantity scored in statistically independent event i (i.e., history $i$ instead of batch $i)$. The equation can be rewritten as:

$$
s_{\bar{X}}=\sqrt{\frac{1}{N-1}\left(\frac{\sum_{i=1}^{N} X_{i}^{2}}{N}-\left(\frac{\sum_{i=1}^{N} X_{i}}{N}\right)^{2}\right)}
$$

where $\mathrm{N}$ is now the number of independent events, i.e., histories. In BEAMnrc and DOSXYZnrc, when using phase space sources, one event or history is defined to be all particle tracks associated with one initial particle (either exiting the accelerator vacuum or from a decaying radioactive source such as ${ }^{60} \mathrm{Co}$ ). It should be noted that the $X_{i}$ may be weighted quantities (if variance reduction techniques, such as bremsstrahlung splitting, are used), while $\mathrm{N}$ is an unweighted quantity, always equal to the total number of independent, or primary, histories.

If we keep track of $\sum_{i=1}^{N} X_{i}^{2}$ and $\sum_{i=1}^{N} X_{i}$ on the fly, then we can calculate the uncertainty at the end of the simulation without the need to store the scored quantity in batches. The problem is that when there are a large number of quantities being scored, it can be very computationally inefficient to evaluate $\sum_{i=1}^{N} X_{i}^{2}$ at the end of each history. To overcome this problem, Sempau et al. [8] outlined the following algorithm for quantity $\mathrm{X}$ :

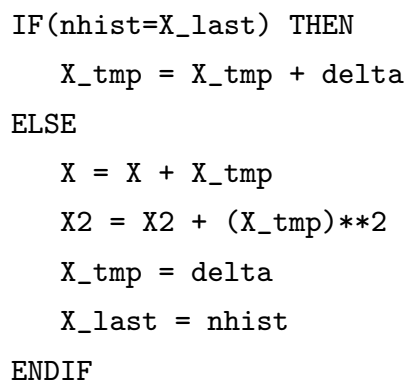

where $\mathrm{X}$ stores $\sum_{i=1}^{N} X_{i}$ during the run, but, after analysis, will store the quantity $\bar{X}$, nhist is the current history number, $X_{-}$last is the number of the last history that contributed to $\mathrm{X}, \mathrm{X}$ _tmp stores the sum of the contributions to $\mathrm{X}$ during the current history, delta is a contribution to $\mathrm{X}$ during the current step, and X2 stores $\sum_{i=1}^{N} X_{i}^{2}$.

Using the algorithm outlined above together with Equation (2), the three main problems with using the batch method are eliminated. The problem of small sample size is eliminated, since $\mathrm{N}$ is now the number of histories and is usually large for a calculation with reasonable statistics. Also, if phase space data from BEAMnrc are used as a source then, by ensuring that $\mathrm{N}$ and nhist only count primary histories (i.e., histories from the original non-phase space source), we properly take into account correlations between incident particles. Finally, the additional 10 or more dimensions required to store the value of $\mathrm{X}$ in each batch have been eliminated and three new scoring arrays, $\mathrm{X}_{-} \mathrm{tmp}, \mathrm{X} 2$ and $\mathrm{X}_{-}$last, have been introduced ( $\mathrm{X}$ is retained from the batch method, but with the batch dimension eliminated). However, $\mathrm{X}$ 
and $\mathrm{X} 2$ have been made double precision to avoid any potential round-off errors and taking into account the other large arrays, the memory requirement for large arrays is only reduced by a factor of 2 compared to when 10 batches were used.

Some quantities output by BEAM are actually ratios of correlated quantities. An example of this is the average energy of photons in a scoring zone, which is given by the total photon energy crossing the zone divided by the total number of photons crossing the zone. These quantities are correlated with each other, and, if output separately, would each have their own uncertainty. In order to estimate uncertainty on these ratios using the history by history method, we use the equation for the fractional uncertainty on a ratio of correlated quantities, $\mathrm{C}=\mathrm{X} / \mathrm{Y}$ :

$$
\frac{s_{\bar{C}}}{\bar{C}}=\sqrt{\left(\frac{s_{\bar{X}}}{\bar{X}}\right)^{2}+\left(\frac{s_{\bar{Y}}}{\bar{Y}}\right)^{2}-\frac{2 \operatorname{cov}(X, Y)}{(N-1)(\bar{X} \bar{Y})}}
$$

where $s_{\bar{X}}$ and $s_{\bar{Y}}$ are the uncertainties on $\bar{X}$ and $\bar{Y}$ estimated using the history by history method outlined above, and $\operatorname{cov}(\mathrm{X}, \mathrm{Y})$ is the covariance of $\mathrm{X}$ and $\mathrm{Y}$, given by:

$$
\operatorname{cov}(X, Y)=\frac{\sum_{i=1}^{N} X_{i} Y_{i}}{N}-\frac{\sum_{i=1}^{N} X_{i} \sum_{i=1}^{N} Y_{i}}{N^{2}}
$$

In order to calculate $\operatorname{cov}(\mathrm{X}, \mathrm{Y})$, we need to keep track of $\sum_{i=1}^{N} X_{i} Y_{i}$ on a history by history basis. This is done on the fly using an algorithm similar to the one given above used to keep track of $\sum_{i=1}^{N} X_{i}^{2}$ and $\sum_{i=1}^{N} X_{i}$. Keeping track of $\sum_{i=1}^{N} X_{i} Y_{i}$ requires an additional REAL $* 8$ variable for each ratio scored.

Note that keeping track of primary histories in a phase space source has made it necessary to modify the format of BEAMnrc phase space files slightly. We now mark the first particle scored in the phase space file from each primary history by setting the particle energy negative. Then, when the phase space file is used as a source, we increment $\mathrm{N}$ and nhist only when a negative energy is read.

Ma et al. [11] have used a similar method to analyse uncertainty in their Monte Carlo dose calculation code, MCDOSE. However, in their approach, the quantity of interest (in this case, energy deposited) is not grouped according to primary history, but according to each energy deposition event. Thus, if a primary history gives rise to two or more charged particles (through interactions and/or variance reduction techniques, such as bremsstrahlung splitting), their method would put the energy deposited by each charged particle in a different group, whereas our technique would put the energy deposited by all resultant charged particles in the same group. It will be seen below that the event-by-event method can give rise to errors in the uncertainty estimate, especially if particles in a phase space source are recycled.

\subsubsection{Latent variance of a phase space file}

Sempau et al.[8] have introduced the term "latent variance of a phase space file" to distinguish between the uncertainty in a dose calculation due to the random nature of the transport in the phantom vs that due to the statistical fluctuations in the phase space file. Thus, a Monte Carlo calculation of the dose distribution generated by a $20 \mathrm{MeV}$ pencil beam of electrons has a statistical uncertainty despite the fact 
that there are no fluctuations in the incident phase space of the source. This is the inherent uncertainty of the dose calculation. This uncertainty will approach zero if the number of histories is increased sufficiently. However, when a finite phase space file is used as a source, the statistical uncertainty on the calculated doses approaches a finite value, independent of how often the phase space file is reused. This value represents the latent variance of the phase space file.

\subsection{Results \& Discussion}

\subsubsection{New vs old methods without recycling}

To see the effect of the improved uncertainty analysis, we simulated an $18 \mathrm{MeV}$ electron beam (field size $=20 \times 20 \mathrm{~cm}$ at $S S D=100 \mathrm{~cm}$ ) from a Clinac $2100 \mathrm{C}$ accelerator and examined uncertainty in dose in a water phantom. This was a 2 -stage process. The accelerator was simulated using BEAMnrc, and a phase space file was scored at the SSD. This phase space file was then used as a source in DOSXYZnrc simulations of a water phantom.

In the BEAMnrc accelerator simulation, 56 million primary histories were used to generate a phase space source containing 51 million particles (including 34 million photons). Range rejection for particles below $3 \mathrm{MeV}$ was used in the accelerator simulation, however, no variance reduction techniques were used which could have led to multiple particles in the same phase space file from the same primary history (e.g., bremsstrahlung splitting). Nonetheless, there can be multiple particles in the phase space file for a given primary history, e.g., the primary electron and bremsstrahlung photons and/or knock-on electrons.

In the DOSXYZnrc simulation of the water phantom 50 million incident particles (of all types) from the phase space source were used. The simulated water phantom itself had dimensions of $20 \times 20 \times 15$ $\mathrm{cm}$, and dose was scored in $1 \times 1 \times 0.5 \mathrm{~cm}$ voxels down the central axis. Region-by-region range rejection for particles below $5 \mathrm{MeV}$ was used in the DOSXYZnrc simulation. This means that a charged particle history was terminated and its energy deposited locally if the particle energy was $\leq 5 \mathrm{MeV}$ and the particle could not make it to the nearest voxel boundary with energy > ECUT, the low-energy threshold for particle transport (700 keV in this case). This range rejection scheme saves $\mathrm{CPU}$ time by cutting short unnecessary transport in the large, off-axis voxels.

Figure 2.1 shows fractional uncertainty in dose vs depth estimated using the new method (grouping energy deposited according to primary history) and using the old method with 10 and 40 batches. A scaled depth-dose curve is also shown for reference. Incident particles from the phase space file were not recycled in these simulations.

It is clear from the figure that the new method estimates a much smoother uncertainty vs depth curve than the batch method, indicating a much lower uncertainty on the uncertainty estimate. It is also clear that increasing the number of arbitrary batches from 10 to 40 results in reduced fluctuations in the uncertainty, albeit at a cost of increasing the memory requirement for the scoring array by a factor of 4. Despite fluctuations, the mean uncertainty in all doses $>0.5 D_{\max }$ estimated using the batch method 


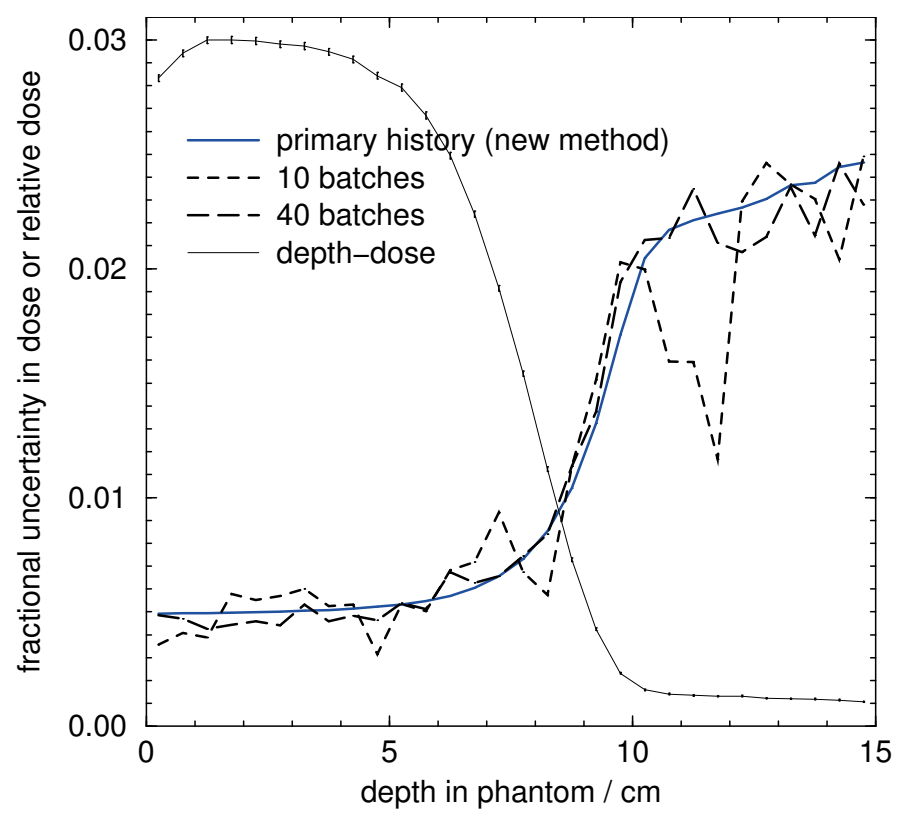

Figure 2.1: Fractional uncertainty in dose calculated on the central axis vs depth for a simulated $18 \mathrm{MeV}$ beam from a Clinac $2100 \mathrm{C}(20 \times 20 \mathrm{~cm}$ field at $S S D=100 \mathrm{~cm})$ in a simulated water phantom. Fractional uncertainties are estimated using the new method (grouping by primary history) and using the old method with 10 and 40 batches. A scaled depth-dose curve is also shown for reference (with uncertainties estimated using the history by history method). Dose was scored in $1 \times 1 \times 0.5 \mathrm{~cm}$ voxels on the central axis.

( $0.55 \%$ using 10 batches and $0.53 \%$ using 40 batches) is in good agreement with that estimated using the new method $(0.54 \%)$. The overall agreement between the 3 methods (aside from fluctuations) implies that any correlation between multiple particles scored in the same primary history, which were placed in separate batches in the old technique, is negligible.

\subsubsection{Effects of recycling}

In many cases, the phase space data available at the bottom of a simulated accelerator may be relatively sparse. In such cases, reducing the inherent uncertainty in the dose calculation to an acceptable level can only be achieved by recycling each particle in the phase space source before moving on to the next one. In order to investigate the effects of particle recycling on the uncertainty, the water phantom simulation was repeated with the same number of incident particles (50 million), but this number was achieved by using only 12.5 million particles from the phase space file with each particle recycled 3 times (each particle used a total of 4 times - the recommended maximum for electron beams) and then by using only 1.8 million particles from the phase space file with each particle recycled 27 times (each particle used a total of 28 times). A DOSXYZnrc technique called smoothing, in which incident particles are reflected 
about the $\mathrm{X}$ axis and the $\mathrm{Y}$ axis before being re-used in the simulation, was also used, but this has little impact on the central axis (see section 2.2.5 below). Note that when a particle is recycled, the number of primary histories (nhist in the new algorithm described in the Introduction) is not incremented. Thus, energy deposited by all occurrences of a particle are grouped into the same primary history.

Figure 2.2 shows the effect of particle recycling on the estimated uncertainty on the central axis. The "no recycling" curves are the same curves as shown for the new method and using 10 batches in Figure 2.1. The uncertainty estimated using the new method clearly indicates that uncertainty increases with the number of times each particle is recycled. The increase is greatest at the phantom surface where the fluctuations reflect the statistical fluctuations of the initial particles hitting just that voxel whereas at depth, the particles from a wider initial area are involved and the in-phantom statistical variations in repeated histories play a role, thereby decreasing the uncertainty.

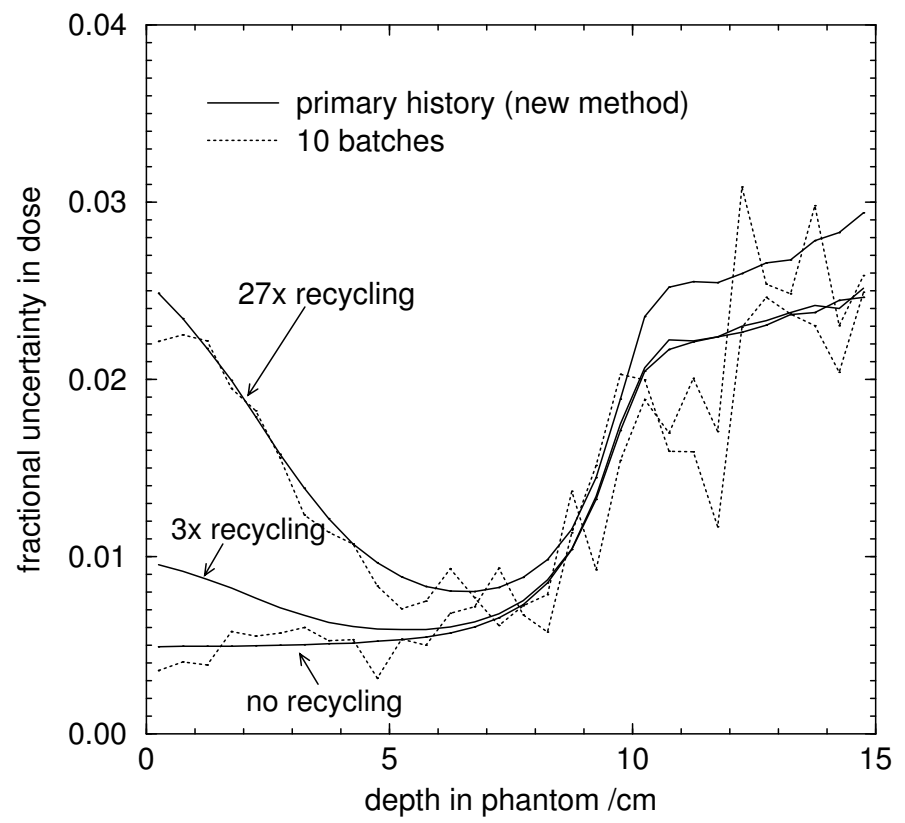

Figure 2.2: Fractional uncertainty in central-axis dose vs depth for a simulated $18 \mathrm{MeV}$ beam from a Clinac $2100 \mathrm{C}(20 \times 20 \mathrm{~cm}$ field at $S S D=100 \mathrm{~cm})$ in a simulated water phantom. Fractional uncertainties estimated using the new method are shown for cases in which incident particles were recycled 27 times, 3 times (the recommended maximum) and not at all. Fractional uncertainties estimated using 10 batches (old method) are shown for the cases in which particles were recycled 27 times and not at all. The total number of particle tracks simulated was 50 million in all cases. Dose was scored in $1 \times 1 \times 0.5 \mathrm{~cm}$ voxels on the central axis.

Recycling incident particles 3 times is equivalent to decreasing the number of primary histories by a factor of 4 (recall the number of primary histories is not incremented when a particle is recycled), and, thus, in an electron beam the uncertainty at the surface of the phantom is expected to increase 
by a factor of $\operatorname{sqrt}(4)=2$ because the uncertainty is dominated by the latent variance of the phase space file. This matches the increase at the surface shown in Figure 2.2. Similarly, when particles are recycled 27 times, the uncertainty at the surface is expected to increase by a factor of $\operatorname{sqr}(28) \sim 5$, which matches the increase shown in the figure. The uncertainty estimated using 10 batches in the case of $27 \times$ recycling shows expected fluctuations, however, similar to the "no recycling" case, the mean uncertainty is in fairly good agreement with that estimated using the new method (mean uncertainty on all doses $>0.5 D_{\max }$ is $1.30 \%$ using 10 batches and $1.38 \%$ using the new method). This agreement validates our previous scheme to account for correlations between recycled particles by putting them in the same statistical batch (accomplished by having the recycling loop inside the statistical batch loop in the code).

When recycling photon sources, the effects on the uncertainty are much less dramatic since, except when highly recycled, photons are not likely to interact in the same voxel and therefore the recycling has little effect. For example, recycling a $13 \mathrm{MV}$ beam 3 times had no effect on the central-axis dose uncertainties and recycling 27 times only increased the uncertainty by about $40 \%$ rather than the factor of 5 seen at the surface in the electron beam (fig 2.2).

The above results indicate that both the new and old methods of statistical analysis took the latent variance of the phase space file (as discussed in the introduction) into account properly. Thus no matter how often a given particle was recycled, the uncertainty would only decrease to a fixed value which reflected the latent variance of the phase space file. This is not the case if the phase space file was restarted rather than each particle being recycled.

\subsubsection{Effects of restarting}

Problems arise in the new and old methods of estimating uncertainty when a phase space file is restarted. This happens automatically upon reaching the end of the source file. A particle that is re-used because of a restart will not be grouped into the same primary history as it was on the previous pass through the phase space file, correlations between re-used particles will be ignored, and the uncertainty will be underestimated, as shown above. Thus, for the purpose of estimating uncertainty, it is recommended that particles be recycled enough times so that the phase space file is only used once. To make this feasible BEAMnrc and DOSXYZnrc offer an option in which the number of times to recycle a particle is automatically calculated based on the number of particles in the phase space source and the number of requested histories. This option may underestimate the number of times to recycle a particle because it cannot take into account particles that do not get used because they are outside the geometry, do not have the correct LATCH value, etc., in which case the phase space source will be restarted. If the source is restarted only once and only a small fraction of it is re-used on the second pass, this is unlikely to have a significant effect on the uncertainty. However, if most of the source is re-used on the second pass, or if it is restarted more than once, then a manually-calculated value for the number of times to recycle particles (based on data from the previous run in which the value was automatically calculated) is recommended. 


\subsubsection{Effects of correlations}

To investigate the effects of correlations, the uncertainty has also been estimated by grouping deposited energy according to incident particle instead of primary history. In this case, nhist in the algorithm described in the Introduction is always incremented, even if the particle is being recycled. This method is similar to the new method but ignores correlations between incident particles when a phase space source is used. When this method was applied to the simulation of the electron beam in water then, regardless of how many times incident particles were recycled, uncertainties were found to agree exactly with those estimated using the new method with no recycling (shown in Figure 2.2). The exact agreement in the case of no recycling indicates that correlations between particles in the phase space file did not affect uncertainty in this electron beam simulation. More importantly, the distinct differences in the cases of recycling indicate that unless correlations introduced by recycling are taken into account, the uncertainty is significantly underestimated because the latent uncertainty of the phase space data is not accounted for.

In electron beams one doesn't expect significant correlations between particles. To investigate the effects on estimated uncertainties of correlations between incident particles (other than those introduced by particle recycling) we used BEAMnrc to simulate a $13.5 \mathrm{MV}$ photon beam $(10 \times 10 \mathrm{~cm}$ field at $\mathrm{SSD}=100$ $\mathrm{cm}$ ) from a Siemens KD2 accelerator and used selective bremsstrahlung splitting (maximum splitting number of 400 , minimum splitting number of 40 ) to enhance photon output. When bremsstrahlung splitting is used, each primary history can potentially generate a large number of photons, all of which are correlated, at the bottom of the accelerator. For this simulation, 25.5 million primary histories were run to generate a phase space file containing 63.5 million particles (63 million of which were photons).

The phase space file generated at the bottom of the photon accelerator was then used as a source in a DOSXYZnrc simulation of a water phantom (dimensions $20 \times 20 \times 60 \mathrm{~cm}$ ). Range rejection (ESAVE=5 $\mathrm{MeV}$ ) was used in all DOSXYZnrc simulations. Initially, we ran 50 million incident particles from the phase space source (no particle recycling) and examined uncertainty on the dose in $2 \times 2 \times 0.5 \mathrm{~cm}$ voxels down the central axis of the phantom. It was found that this did not include enough of the incident beam field to show any effects of correlations between incident particles. By increasing the size of the central-axis voxels to $10 \times 10 \times 0.5 \mathrm{~cm}$, with a corresponding decrease in the number of incident particles to 1.5 million (no recycling), we were able to see the effects of correlations.

Figure 2.3 shows the estimated uncertainty in dose vs depth for the photon beam simulation. Uncertainties were estimated by grouping deposited energy according to primary history (the new method), by splitting the run into 40 statistical batches (the old method), and by grouping energy deposited according to incident particle (similar to new method but ignoring correlations). A scaled depth dose curve is also shown in the figure for reference. It is interesting to note that the 1.5 million incident particles used in this simulation was found to represent only 209,196 primary histories, indicating a fairly high degree of correlation between photons in the phase space source.

From Figure 2.3 it is clear that, similar to the electron beam example, the new method of estimating uncertainty yields a much smoother uncertainty vs depth curve than when 40 batches are used, indicating 


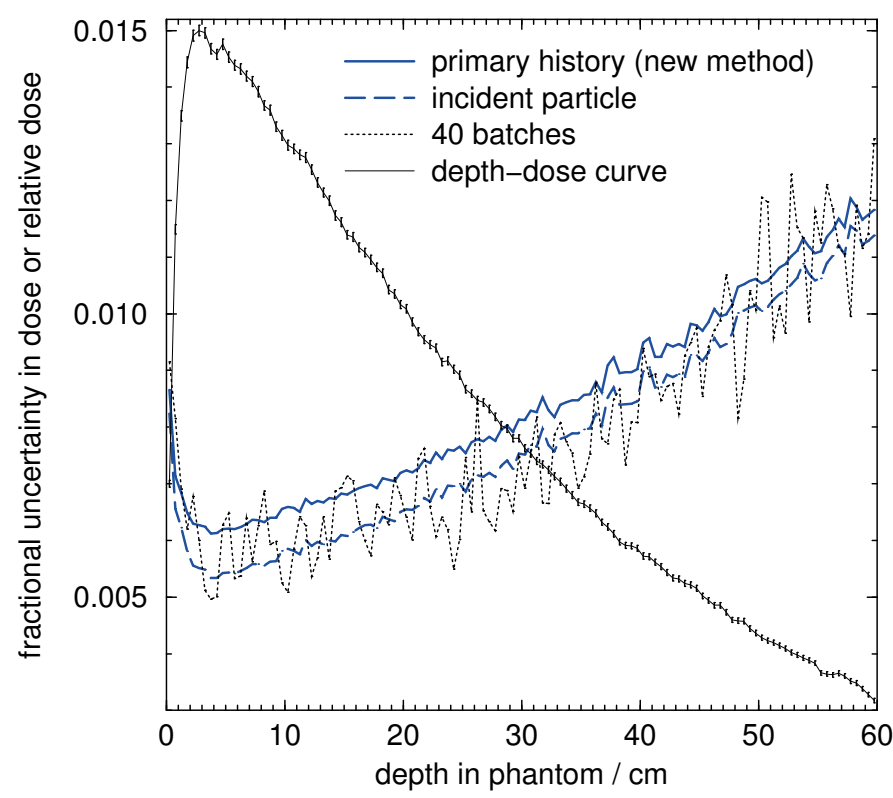

Figure 2.3: Fractional uncertainty in central-axis dose vs depth for a simulated $13.5 \mathrm{MV}$ photon beam from a Siemens KD2 accelerator $(10 \times 10 \mathrm{~cm}$ field at $S S D=100 \mathrm{~cm})$ in a simulated water phantom $(20 \times 20 \times 60 \mathrm{~cm})$. Uncertainties were estimated three ways: using the new method (grouping by primary history); using 40 statistical batches; and using a method similar to the new method but which ignores correlations between incident particles (grouping by incident particle). A scaled depth-dose curve is also shown for reference (with uncertainties estimated using the history by history method). Dose was scored in $10 \times 10 \times 0.5 \mathrm{~cm}$ voxels on the central axis.

a much lower uncertainty on the uncertainty estimate. The figure also shows that ignoring correlations between incident photons by grouping according to incident particle results in a consistent underestimate of the uncertainty (although the curve is still smooth). The batch method also ignores correlations between particles and in the figure it can be seen that it also tends to underestimate uncertainty. The mean uncertainty on all doses $>0.5 D_{\max }$ estimated using 40 batches is $0.64 \%$, which is in better agreement with the mean of $0.63 \%$ estimated by grouping according to incident particle than it is with the mean of $0.70 \%$ estimated using the new method.

One surprising result from Figure 2.3 is the similarity between the shape of the uncertainty vs depth curve estimated using the new method and that estimated by grouping according to incident particle, even the small fluctuations. In fact, they appear to be the same curve, just translated in the Y-direction. This similarity was also found to exist in the absolute uncertainties estimated using these two methods (i.e., once fluctuations in dose had been factored out). This indicates that there are very few cases in which multiple photons originating from the same primary history lead to energy deposited in the same voxel. Thus, differences between uncertainty estimated using the new method and by grouping according to 
incident particle are mainly due to differences in the number $\mathrm{N}$ in Equation (2). Using the new method, $\mathrm{N}$ is the number of primary histories, while grouping according to incident particle, $\mathrm{N}$ is the number of incident particles.

\subsubsection{Effects of Smoothing on Uncertainties}

When recycling or restarting a phase space source in DOSXYZnrc, one has the option of using a routine which makes use of the symmetry in many beams to re-distribute the phase-space particles to 3 symmetrical positions $((\mathrm{x}, \mathrm{y})$ with direction cosines $(\mathrm{u}, \mathrm{v})$ goes to $(-\mathrm{x}, \mathrm{y})$ with $(-\mathrm{u}, \mathrm{v})$ etc). This means that, away from the axes, one can effectively gain a factor of 4 increase in the number of different initial particles incident on a particular region since one gets them from all four quadrants. Unfortunately, close to the central axis this has little value since all four locations are very close to each other. This is clear in figure 2.2 where the uncertainty on the central-axis dose increased by a factor of two near the surface, even with smoothing turned on. At greater depths, the recycling causes only a small increase in the uncertainty, but the accelerator simulation required four times fewer histories. We find that for a voxel element away from the axes, with smoothing on the uncertainty estimate for the 3 times recycling case is the same as in the no recycle case. Hence, for electron beams the use of the smoothing option with the recycling option clearly has a positive benefit in much of the volume, but has no benefit on the central axis at the surface, and also a reduced benefit along the $\mathrm{x}$ - and $\mathrm{y}$-axes. In any case, the uncertainty estimates are accurate.

For photon beams the effects of smoothing are not so dramatic since the effects of recycling are not as dramatic as discussed above. Nonetheless, the trends are similar to the effects with electron beams, namely smoothing is most effective away from the axes.

\subsubsection{Ratios of correlated quantities}

In order to determine how well the history by history method is able to estimate uncertainties in ratios of correlated quantities (using Equations 2.3 and 2.4 above), we examined the $\chi^{2}$ per degree of freedom for average photon energies at the bottom of a simulated accelerator. As mentioned in Section 2.1.2 average photon energy is a ratio of the correlated quantities total photon energy and number of photons.

We performed 20 separate simulations (500,000 histories each) of a generic $16 \mathrm{MV}$ photon accelerator (field size $=10 \times 10 \mathrm{~cm}$ at $S S D=100 \mathrm{~cm}$ ) and calculated the average photon energies (with uncertainties) for photons crossing 10 scoring zones at the SSD. The scoring zones were square "rings" centred on the beam axis with mid-points at a distance $0,1.5,2.5,3.5,4.5,5.5,6.5,7.5,8.5$ and $9.5 \mathrm{~cm}$ from the beam axis. We then estimated the $\chi^{2}$ per degree of freedom in each scoring zone using the equation:

$$
\frac{\chi^{2}}{d f}=\frac{1}{N-1} \sum_{i=1}^{N} \frac{\left(E_{i}-\bar{E}\right)^{2}}{s_{E_{i}}^{2}}
$$

where $\mathrm{N}$ is the number of simulations (20 in this case), $E_{i}$ is the average photon energy crossing the scoring zone in simulation $\mathrm{i}, s_{E_{i}}$ is the uncertainty on $E_{i}$, and $\bar{E}$ is the photon energy crossing the scoring zone averaged over all $\mathrm{N}$ simulations. 
The results are shown in Figure 2.4. If the uncertainty is estimated accurately then we expect $\chi^{2} / d f$ to $\mathrm{be} \approx 1$. A $\chi^{2} / d f<<1$ indicates that the uncertainty has been overestimated, and a $\chi^{2} / d f>>1$ indicates an underestimate of the uncertainty. Figure 2.4 shows that $\chi^{2} / d f$ is $\approx 1$ for most scoring zones.

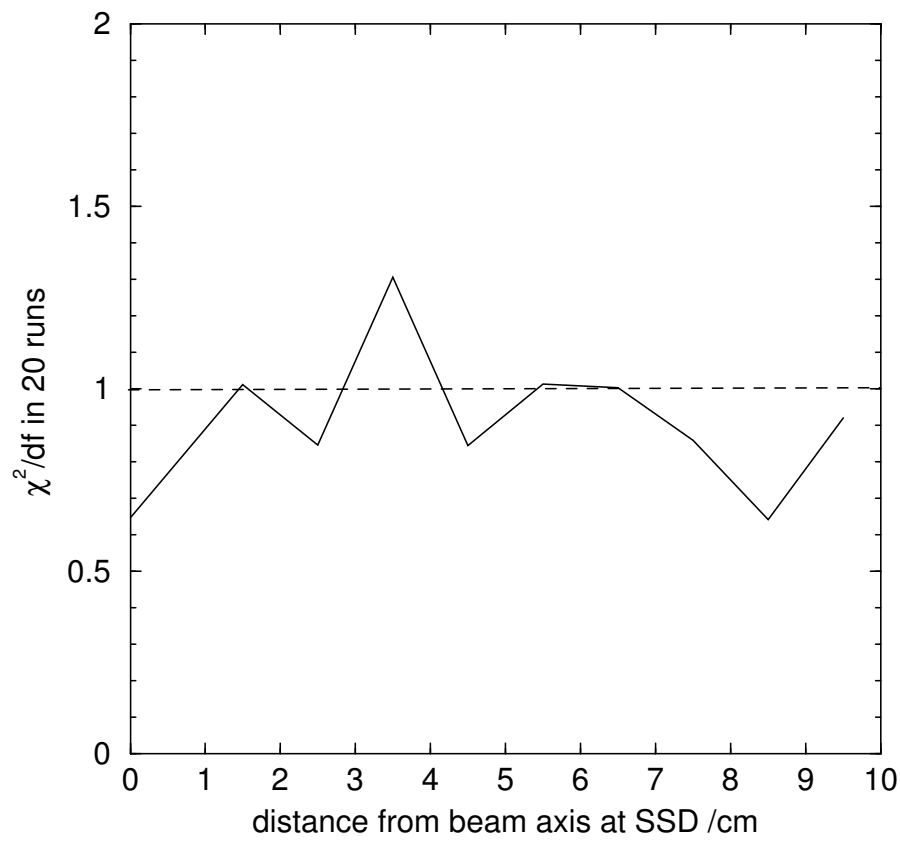

Figure 2.4: $\chi^{2}$ per degree of freedom for average photon energy at the SSD $(100 \mathrm{~cm})$ of a generic $16 \mathrm{MV}$ photon accelerator evaluated over 20 separate simulations (500,000 histories each). Scoring zones in which average photon energies (and their uncertainties) were calculated were square "rings" centred on the beam axis. Mid-points of the square rings were at $0,1.5,2.5,3.5,4.5,5.5,6.5,7.5,8.5$ and $9.5 \mathrm{~cm}$ from the beam axis. Field size was $10 \times 10 \mathrm{~cm}$ at the SSD.

We have found that for a simulation with extreme weight variations, the $\chi^{2} / d f$ was about 1 except for one or two scoring zones where it was about 2 , caused by one or two outliers in the 20 calculations. We take this as an indicator of using too much biasing, causing inconsistent results due to "fat" particles with very large weights relative to most particles. This could possibly even be developed into a diagnostic tool for the variance reduction techniques.

\subsubsection{A note on parallel runs}

If one has multiple machines running on a network, then it is possible to split BEAMnrc and DOSXYZnrc simulation up into a number of parallel jobs[4]. When a simulation that uses a phase space source is split up into $\mathrm{n}$ multiple jobs, then the phase space source is automatically divided into $\mathrm{n}$ equal partitions. Each 
job uses a separate partition. Thus, job i uses particle numbers that fall in the range:

$$
(i-1) \times \frac{\text { nshist }}{n}<\text { nnphsp }<=i \times \frac{\text { nshist }}{n}
$$

where nshist is the total number of particles in the phase space source and nnphsp is the number of the particle used. This partitioning scheme was adopted to ensure that the entire phase space source is adequately sampled over all of the parallel jobs.

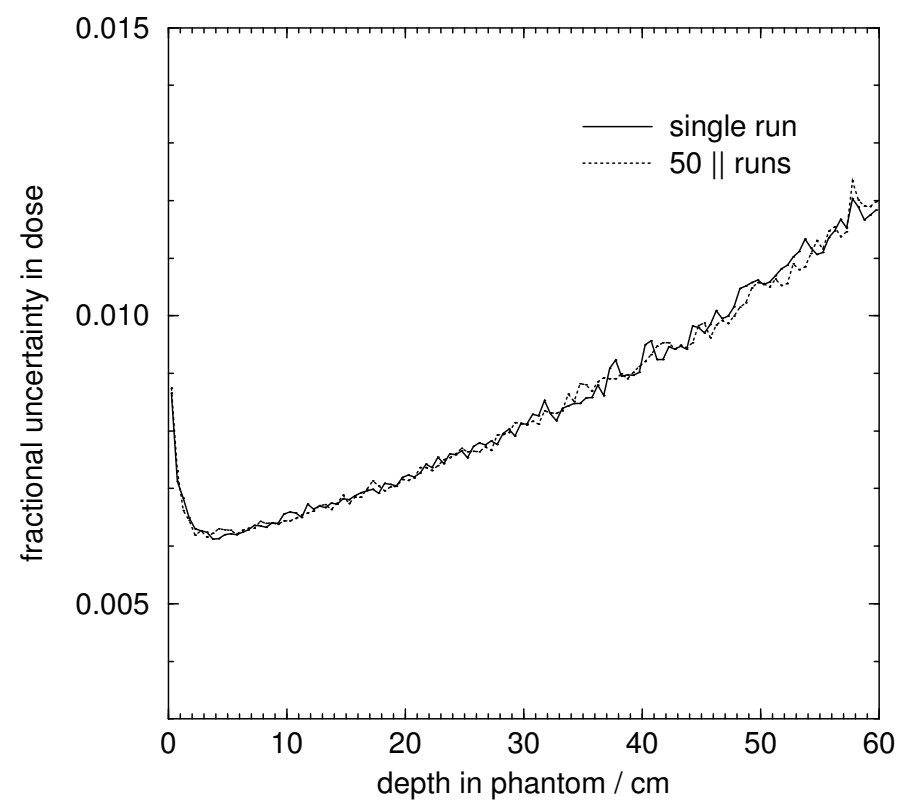

Figure 2.5: Fractional uncertainty in central-axis dose vs depth for a simulated 13.5 MV photon beam from a Siemens KD2 accelerator $(10 \times 10 \mathrm{~cm}$ field at $S S D=100 \mathrm{~cm})$ in a simulated water phantom $(20 \times 20 \times 60 \mathrm{~cm})$. Uncertainties are shown estimated using the new method when the simulation was performed in a single run (1.5 million incident particles) and when the simulation was divided into 50 parallel jobs $(30,000$ incident particles each). Dose was scored in $10 \times 10 \times 0.5 \mathrm{~cm}$ voxels on the central axis.

Partitioning of phase space sources potentially interferes with the new method of estimating uncertainties because it may split up particles generated by the same primary history (correlated) and use them in different jobs. When the jobs are recombined for final analysis, any quantity scored by these particles will be grouped as if it originated in two or more different primary histories, instead of just one. This introduces the possibility of some correlations being ignored and an underestimate of the uncertainty as discussed above.

In order to observe the effects of partitioning a phase space source on uncertainty, we split the simulation of the 13.5 MV photon beam in water (described above) into 50 parallel jobs. Each job simulated 
30,000 histories $\left(1.5 \times 10^{6} / 50\right)$. Division into 50 jobs represents an extreme case, and increases the chances that correlated groups of photons in the phase space source will be broken up by partitioning the file.

Figure 2.5 shows the fractional uncertainty in dose vs depth estimated after recombining the 50 parallel jobs, along with the uncertainty estimated when the simulation was performed in a single run (same as solid line Figure 2.3 above). The figure shows no significant difference between the uncertainties in the two cases. The mean uncertainty of all doses $>0.5 D_{\max }$ estimated after recombining the 50 runs is $0.697 \%$, which is in good agreement with the mean uncertainty of $0.700 \%$ estimated in the single run. Thus, we conclude that, in general, partitioning of a phase space source has no effect on the estimated uncertainty.

\subsection{Conclusions}

A new method for better estimates of uncertainty in the BEAMnrc and DOSXYZnrc codes has been introduced. Scored quantities are now grouped according to primary history and not statistical batches as before. This new method eliminates the problems of fluctuations in estimated uncertainty due to small sample size (i.e., small number of batches). It also eliminates one dimension from the arrays that score quantities of interest, resulting in a decrease in the memory required by the scoring arrays by a factor of about 2 . This new method can easily be adapted to estimate the uncertainty on ratios of correlated quantities as well.

The new method also accounts for correlations between incident particles in a phase space source by grouping incident particles according to the primary histories that generated them. This has been accomplished by changing the format of phase space files slightly, so that the first particle scored from a new primary history is marked by setting its energy negative.

Recycling incident particles (i.e., using the same particle as an incident particle many times immediately after reading it), which is often necessary when phase space data are sparse, introduce the correlations with the most significant effect on uncertainty. Failure to take into account these correlations can lead to a significant underestimate of the uncertainty, basically because it ignores the statistical fluctuations inherent in the phase space file. On the other hand, when correlations are taken into account then, even if particles are recycled many times, the uncertainty will always reflect the uncertainty in the phase space data itself. It was found that the batch method also successfully accounted for correlations introduced by recycling by placing all recurrences of a particle in the same statistical batch. Problems occur with both the new method and the batch method of estimating uncertainties if a phase space source is restarted. In the case of the new method, a particle that gets re-read after the phase space file has been restarted will not be grouped in the same primary history as it was on the previous pass. In the case of the batch method, there is no guarantee that the particle re-read after a restart will be put in the same batch as it was on the previous pass. Thus, restarting can cause uncertainty to be underestimated, and we recommend recycling particles enough times to avoid restarting a phase space source (as well a reducing needless file re-reading). 
Other correlations between particles in a phase space source include those that occur "naturally" (e.g., a primary history that undergoes interactions leading to more than one particle in the phase space source) and those that occur due to variance reduction techniques, such as bremsstrahlung splitting. These correlations are taken into account by the new method, but not by the batch method of estimating uncertainty. We examined the case of a photon beam generated by splitting bremsstrahlung photons up to 400 times (using selective bremsstrahlung splitting) in water and found that the batch method did, in fact, underestimate uncertainty. However, the large bremsstrahlung splitting number and the fact that significant differences were not noticeable until the voxels in the water phantom had been enlarged (to $10 \times 10 \times 0.5 \mathrm{~cm}$ ) to encompass most of the incident beam leads to the conclusion that, in most cases, these correlations do not play a role in the uncertainty estimate.

It should be noted that the event-by-event technique used by Ma et el. [11] and mentioned in the Introduction will not take into account correlations between incident particles in a phase space source. This is because this technique does not trace energy deposited back to primary histories. It is even more extreme than the case discussed in section 2.2.4 in which energy deposition was grouped according to particle incident on the phantom rather than by initial history. As shown there, this latter case is not likely to be a problem when correlations are due to interactions and/or variance reduction techniques. However, if correlations are introduced by recycling particles, then grouping by particles incident on the phantom is expected to significantly underestimate uncertainty since it ignores the latent variance of the phase space file. The techniques used by Ma et al. will suffer this same problem.

One closing observation is that issues about the latent variance of a phase space file are overcome, or at least highly mitigated, by using beam characterization models[12]. These models do not need to recycle particles and hence avoid the associated problems. Of course there is still some uncertainty to be associated with the models themselves, but as shown elsewhere, the use of the model greatly reduces these effects[12].

The history by history method has also recently been added to the standard EGSnrc user-codes which are distributed by NRC[13].

\subsection{Bibliography}

[1] D. W. O. Rogers, B. A. Faddegon, G. X. Ding, C.-M. Ma, J. Wei, and T. R. Mackie. BEAM: A Monte Carlo code to simulate radiotherapy treatment units. Med. Phys., 22:503 - 524, 1995.

[2] D. W. O. Rogers. Publications using or about BEAM or the OMEGA project . http://www.irs.inms.nrc.ca/inms/irs/BEAM/bibliog/omega_pubs.html.

[3] C.-M. Ma, D. W. O. Rogers, and B. Walters. DOSXYZnrc Users Manual. NRC Report PIRS $509 b(\operatorname{revF}), 2001$.

[4] D. W. O. Rogers, C.-M. Ma, G. X. Ding, B. Walters, D. Sheikh-Bagheri, and G. G. Zhang. BEAMnrc Users Manual. NRC Report PIRS 509(a)revF, 2001. 
[5] I. Kawrakow. Accurate condensed history Monte Carlo simulation of electron transport. I. EGSnrc, the new EGS4 version. Med. Phys., 27:485 - 498, 2000.

[6] I. Kawrakow and D. W. O. Rogers. The EGSnrc Code System: Monte Carlo simulation of electron and photon transport. Technical Report PIRS-701, National Research Council of Canada, Ottawa, Canada, 2000.

[7] J. Sempau, S. J. Wilderman, and A. F. Bielajew. DPM, a fast, accurate Monte Carlo code optimized for photon and electron radiotherapy treatment planning dose calculations. Phys. Med. Biol., 45: $2263-2291,2000$.

[8] J. Sempau, A. Sánchez-Reyes, F. Salvat, H. Oulad ben Tahar, S. B. Jiang, and J. M. Fernández-Varea. Monte Carlo simulation of electron beams from an accelerator head using PENELOPE. Phys. Med. Biol., 46:1163 - 1186, 2001.

[9] J. Briesmeister. MCNP-A general purpose Monte Carlo code for neutron and photon transport, Version 3A. Los Alamos National Laboratory Report LA-7396-M (Los Alamos, NM), 1986.

[10] P. Andreo. Monte Carlo techniques in medical radiation physics. Phys. Med. Biol., 36:861 - 920, 1991.

[11] C.-M. Ma, J. S. Li, T Pawlicki, S. B. Jiang, J. Deng, M. C. Lee, T. Koumrian, M. Luxton, and S. Brain. A Monte Carlo dose calculation tool for radiotherapy treatment planning. Phys. Med. Biol., 47:1671 $-1690,2002$.

[12] C.-M. Ma, B. A. Faddegon, D. W. O. Rogers, and T. R. Mackie. Accurate characterization of Monte Carlo calculated electron beams for radiotherapy. Med. Phys., 24:401 - 416, 1997.

[13] D. W. O. Rogers, I. Kawrakow, J. P. Seuntjens, and B. R. B. Walters. NRC User Codes for EGSnrc. Technical Report PIRS-702, National Research Council of Canada, Ottawa, Canada, 2000. 



\section{III}

\section{A "HOWFARLESS" option to increase efficiency of homogeneous phantom calculations with DOSXYZnrc}




\begin{abstract}
This paper describes a "HOWFARLESS" transport option, which has been added to DOSXYZnrc to increase the efficiency of beam commissioning calculations in homogeneous phantoms. The algorithm speeds up charged particle transport by only considering the distance to the extreme outer boundaries of the phantom, thus eliminating the need to stop at voxel boundaries. Dose is deposited by approximating the total curved charged particle steps by two straight-line steps joined at a hinge point. Good agreement with normal simulations is achieved at all beam energies and for all practical maximum step lengths with a 1:1 mixture of approximations based on the initial position/direction of the particle and on its final position/direction. Use of the "HOWFARLESS" option in phantom calculations for $6 \mathrm{MV}$ and $18 \mathrm{MV}$ photon beams $\left(10 \times 10 \mathrm{~cm}^{2}\right.$ and $40 \times 40 \mathrm{~cm}^{2}$ fields) from BEAMnrc-simulated accelerators increases the efficiency at the optimum photon splitting number by a factor of 2.9-5.4 when the exact EGSnrc boundary crossing algorithm (BCA) is used and by $51-89 \%$ when the faster PRESTA-I BCA is employed. The efficiency gain due to the "HOWFARLESS" transport option increases with increasing beam energy and decreases with increasing field/dose voxel size. Efficiency improvement is greater when the efficiency of the particle source itself is not a factor, and in such cases the "HOWFARLESS" option improves the DOSXYZnrc efficiency by up to a factor of 13.1 (exact BCA) or 3.5 (PRESTA-I BCA) for photon beams, and up to a factor of 17.2 (exact BCA) or 5.2 (PRESTA-I BCA) for electron beams.
\end{abstract}




\subsection{Introduction}

With the increased interest in using Monte Carlo simulations for radiotherapy treatment planning[1], there is a concurrent need to commission the beam models used in the simulations by comparing Monte Carlo calculated doses in water phantoms to actual measurements (see e.g. the report by the AAPM Task Group 105[1], and the review articles on Monte Carlo treatment head simulations by Verhaegen and Seuntjens[2] and by Ma and Jiang[3]). Such beam commissioning calculations often require long simulation times, thus providing a motivation for the development of faster Monte Carlo transport techniques.

The BEAMnrc package[4] is a system based on EGSnrc $[5,6]$ that has been used extensively to model radiation treatment sources. The package includes DOSXYZnrc[7], a Monte Carlo code for calculating dose distributions in a phantom composed of rectilinear voxels. DOSXYZnrc calculations can be coupled to BEAMnrc accelerator simulations either via a phase space output from the accelerator simulation or, since the distribution of BEAMnrc06, by using the entire BEAMnrc accelerator simulation as a source in the DOSXYZnrc calculation. The latter approach has many advantages, chief among them being the elimination of intermediate phase space files, with little loss of efficiency[8]. DOSXYZnrc need not be used in conjunction with BEAMnrc and can be employed for stand-alone dose calculations with other user-defined sources.

Significant improvements in BEAMnrc/DOSXYZnrc simulation efficiency have been reported in the recent literature. The Directional Bremsstrahlung Splitting (DBS) technique[9] speeds up BEAMnrc treatment head simulations by up to a factor of 25 compared to Uniform Bremsstrahlung Splitting (UBS), the variance reduction technique utilized in most published investigations. When DBS in the BEAMnrc treatment head source is combined with photon splitting in the DOSXYZnrc phantom, an additional factor of up to 6.5 can be achieved[8]. Since most of the simulation time is spent in phantom transport, further improvements of the simulation efficiency in the phantom are more important for increasing the overall calculation speed than improvements of the treatment head efficiency.

In standard EGSnrc simulations, a single particle step cannot take it beyond the boundaries of the current voxel. This restriction, dictated by the specific implementation of charged particle transport in EGSnrc and also present in most other general purpose Monte Carlo codes, is not required in the homogeneous phantoms used for beam commissioning calculations. Provided that an algorithm can be determined for accurate deposition of energy along a charged particle path, particles may be allowed to step beyond voxel boundaries in a homogeneous phantom, thereby saving a significant amount of computation time.

This study investigates the accuracy and efficiency of a new charged particle transport option implemented in DOSXYZnrc in which single particle steps are allowed to cross voxel boundaries and are restricted only by the extreme outer boundaries of the phantom. In EGSnrc the subroutine providing the distance to the next region boundary along the current direction of motion is called HOWFAR. Therefore, simulations involving the new transport algorithm will be referred to as "HOWFARLESS", as opposed to standard EGSnrc simulations with HOWFAR. The accuracy of the "HOWFARLESS" algorithm is examined in $6 \mathrm{MeV}$ and $20 \mathrm{MeV}$ monoenergetic electron beams and in $6 \mathrm{MV}$ and $24 \mathrm{MV}$ 
photon beams with energy spectra identical to those published by Mohan et al[10] for Varian Clinac- 6 (6 MV) and Clinac-2500 (24 MV) accelerators. Efficiency is investigated in the aforementioned beams and in $6 \mathrm{MV}$ photon beams $\left(10 \times 10 \mathrm{~cm}^{2}\right.$ and $40 \times 40 \mathrm{~cm}^{2}$ fields) from a BEAMnrc-simulated Elekta SL25 accelerator and an $18 \mathrm{MV}$ photon beam $\left(10 \times 10 \mathrm{~cm}^{2}\right.$ field) from a BEAMnrc-simulated Siemens KD2 accelerator. For the BEAMnrc- simulated accelerators, the efficiency of the dose calculation is examined using phase space sources and BEAMnrc simulation sources. In the case of photon beams, the efficiency of the "HOWFARLESS" option is studied in combination with photon splitting in DOSXYZnrc, which has been shown to increase the efficiency of dose calculations by a factor of up to 6.5[8].

Section 3.2 describes the algorithm used to approximate the total curved path of a single charged particle step using two straight-line steps joined at a hinge point. This is necessary for accurate deposition of dose in the voxels intersected by the step. The section discusses how the hinged-step can be calculated using either the particle's known initial direction or its known final direction. Section 3.3 gives a detailed description of how the "HOWFARLESS" algorithm is implemented in DOSXYZnrc. Section 3.4.1 describes the DOSXYZnrc phantom simulations used to determine the accuracy of the dose deposition algorithm, and Section 3.4.2 describes the calculations used to determine the efficiency of the "HOWFARLESS" method vs calculations with HOWFAR. Results are presented in Section 3.5 which is broken down as follows:

- Section 3.5.1 presents accuracy results, including a discussion of the effects of the exact vs PRESTAI boundary crossing algorithm on the accuracy of calculations with HOWFAR and "HOWFARLESS (Section 3.5.1.1), and results showing the accuracy of "HOWFARLESS" calculations compared to calculations with HOWFAR as a function of the maximum allowable electron step length (Smax) and the ratio of hinged-steps based on the initial particle direction to those based on the final direction (Section 3.5.1.2).

- Section 3.5.2 shows efficiency results of dose calculations, including the efficiency of calculations with "HOWFARLESS" as a function of Smax (Section 3.5.2.1), and the efficiency of "HOWFARLESS" vs HOWFAR in dose calculations for various beams, including realistic photon beams simulated with BEAMnrc, simple photon beams with energy spectra, and monoenergetic electron beams (Section 3.5.2.1).

Concluding remarks appear in Section 3.6.

\subsection{Theory: approximation of curved charged particle steps by hinged steps}

In the "HOWFARLESS" option, for the purposes of dose deposition the total curved path of a charged particle step is approximated by two straight-line steps connected at a hinge point. The method of approximating a curved charged particle step by a hinged step has been in use since 1995 in the PENELOPE[11] Monte Carlo radiation transport code, since 1997 in the VMC series of codes[12-16], and since 2000 by Sempau et al[17] in their DPM Monte Carlo code. In these codes, the hinge is placed at a randomly selected point on the particle path (with different approaches used in PENELOPE, DPM and 
$\mathrm{XVMC} / \mathrm{VMC}++$ to select the random hinge point), and the resultant hinged step is the basis for their condensed history transport. In DOSXYZnrc, however, the more accurate EGSnrc transport algorithm[5, $6,18]$ is utilized, and the initial and final positions and directions of the charged particle, as well as the total curved path length between the two points, are known. Thus, the role of the hinged step approximation in the "HOWFARLESS" option is strictly to recreate a step already taken for the purposes of dose deposition. Moreover, the hinge point is not randomly selected but is uniquely determined by either the initial direction or the final direction of the particle. Figure 3.1 shows the relationship between the two possible hinged steps and the full, condensed history charged particle step.

HOWFAR

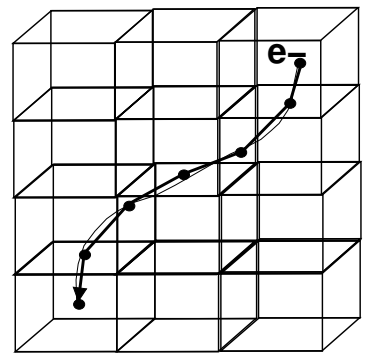

"HOWFARLESS"

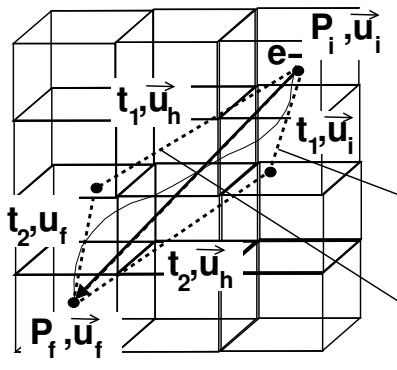

- USTEP<smiles>[Te]=[Te]</smiles>

forward

hinged

reverse

hinged

step

Figure 3.1: Illustration of charged particle steps taken with HOWFAR (left figure) and with the "HOWFARLESS" option (right figure). In both cases, the particle travels over the same total curved pathlength, $t_{v}$ (thin solid curve). In HOWFAR, $t_{v}$ is approximated by condensed history steps which have a maximum length, USTEP (thick solid lines), restricted by the distance to the next voxel boundary. With "HOWFARLESS", USTEP is only restricted by the extreme outer boundaries of the phantom, so a particle can take a single step across voxel boundaries. Although the step need not track $t_{v}$, the curved path must be approximated for the purpose of dose deposition once the step has been taken. This is done using two straight-line segments joined at a hinge point (hinged steps). The lengths of the segments, $\mathrm{t}_{1}$ and $\mathrm{t}_{2}$, and the hinge direction vector, $\overrightarrow{u_{h}}=\left(\mathrm{u}_{h}, \mathrm{v}_{h}, \mathrm{w}_{h}\right)$, are calculated using the known particle initial position, $\mathrm{P}_{i}=\left(\mathrm{x}_{i}, \mathrm{y}_{i}, \mathrm{z}_{i}\right)$, its known final position, $\mathrm{P}_{f}=\left(\mathrm{x}_{f}, \mathrm{y}_{f}, \mathrm{z}_{f}\right)$, and either its known initial direction, $\overrightarrow{u_{i}}=\left(\mathrm{u}_{i}, \mathrm{v}_{i}, \mathrm{w}_{i}\right)$, as in Equations (3.2) and (3.4), to create a forward hinged step or the particle's known final direction, $\overrightarrow{u_{f}}=\left(u_{f}, v_{f}, w_{f}\right)$, as in Equations (3.5) and (3.6), to create a reverse hinged step. Note that in forward hinged steps the second segment of the step $\left(t_{2}\right)$ has direction $\overrightarrow{u_{h}}$, while in reverse hinged steps, the first segment of the step $\left(t_{1}\right)$ has direction $\overrightarrow{u_{h}}$.

For a step of known total curved path length, $t_{v}$, and a hinged step based on the known particle initial direction cosines, $\left(u_{i}, v_{i}, w_{i}\right)$, the final particle position, $\left(x_{f}, y_{f}, z_{f}\right)$, is given by:

$$
\begin{aligned}
& x_{f}=x_{i}+t_{1} u_{i}+\left(t_{v}-t_{1}\right) u_{h} \\
& y_{f}=y_{i}+t_{1} v_{i}+\left(t_{v}-t_{1}\right) v_{h} \\
& z_{f}=z_{i}+t_{1} w_{i}+\left(t_{v}-t_{1}\right) w_{h}
\end{aligned}
$$

where $t_{1}$ is the length of the first segment of the hinged step, and $\left(u_{h}, v_{h}, w_{h}\right)$ are the direction cosines of 
the second segment of the hinged step (length $=t_{2}=t_{v}-t_{1}$ ). Note that the direction cosines of the first segment of the hinged step are the known initial direction cosines, $\left(u_{i}, v_{i}, w_{i}\right)$. Rearranging Equation 3.1 to solve for $\left(u_{h}, v_{h}, w_{h}\right)$ gives:

$$
\begin{aligned}
u_{h}=\frac{\Delta x-t_{1} u_{i}}{\left(t_{v}-t_{1}\right)}, & \Delta x=x_{f}-x_{i} \\
v_{h}=\frac{\Delta y-t_{1} v_{i}}{\left(t_{v}-t_{1}\right)}, & \Delta y=y_{f}-y_{i} \\
w_{h}=\frac{\Delta z-t_{1} w_{i}}{\left(t_{v}-t_{1}\right)}, & \Delta z=z_{f}-z_{i}
\end{aligned}
$$

Substituting these expressions into the normalization condition for the direction,

$$
u_{h}^{2}+v_{h}^{2}+w_{h}^{2}=1
$$

and solving for $t_{1}$ gives:

$$
t_{1}=\frac{t_{v}^{2}-(\Delta x)^{2}-(\Delta y)^{2}-(\Delta z)^{2}}{2 t_{v}\left(1-\frac{\left(u_{i} \Delta x+v_{i} \Delta y+w_{i} \Delta z\right)}{t_{v}}\right)}
$$

Once Equation (3.4) has been solved, then the value of $t_{1}$ can be used in Equation (3.2) to determine $\left(u_{h}, v_{h}, w_{h}\right)$. Note that $\left(u_{h}, v_{h}, w_{h}\right) \neq\left(u_{f}, v_{f}, w_{f}\right)$.

The hinged step can also be based on the known particle final direction cosines, $\left(u_{f}, v_{f}, w_{f}\right)$. In this case, $\left(u_{f}, v_{f}, w_{f}\right)$ are the direction cosines for the second segment of the hinged step (length $\left.=t_{2}\right)$. Solving for $\left(x_{f}, y_{f}, z_{f}\right)$ in a manner similar to that described above gives:

$$
\begin{aligned}
u_{h} & =\frac{\Delta x-t_{2} u_{f}}{\left(t_{v}-t_{2}\right)} \\
v_{h} & =\frac{\Delta y-t_{2} v_{f}}{\left(t_{v}-t_{2}\right)} \\
w_{h} & =\frac{\Delta z-t_{2} w_{f}}{\left(t_{v}-t_{2}\right)}
\end{aligned}
$$

and using the condition given by Equation (3.3) to solve for $t_{2}$ yields

$$
t_{2}=\frac{t_{v}^{2}-(\Delta x)^{2}-(\Delta y)^{2}-(\Delta z)^{2}}{2 t_{v}\left(1-\frac{\left(u_{f} \Delta x+v_{f} \Delta y+w_{f} \Delta z\right)}{t_{v}}\right)} .
$$

Note that the direction cosines, $\left(u_{h}, v_{h}, w_{h}\right)$, calculated using Equation (3.5) now apply to the first segment of the hinged step (length $=t_{1}=t_{v}-t_{2}$ ).

To differentiate between the two different hinged steps in discussions below, a hinged step calculated using Equations (3.2) and (3.4) will be referred to as a "forward" hinged step, and a hinged step calculated using Equations (3.5) and (3.6) will be called a "reverse" hinged step. 


\subsection{The "HOWFARLESS" algorithm}

This section gives a detailed description of the method of particle transport when the "HOWFARLESS" option is used.

\subsubsection{Changes to the HOWFAR and HOWNEAR subroutines}

HOWFAR is a subroutine that determines the distance along the particle's directory to the nearest region boundary and is used to determine the maximum allowable step length. HOWNEAR computes the perpendicular distance to the nearest region boundary in any direction. This perpendicular distance is used: 1) to determine if a charged particle is sufficiently close to a boundary (within a distance called "skin depth" [5, 6]) that single-scattering transport should be used instead of condensed history steps; 2 ) to determine if HOWFAR needs to be called (if the planned step length is greater than the perpendicular distance); and 3) by the range rejection algorithm (if turned on) to determine if the particle can reach the nearest region boundary with energy higher than the transport cutoff energy (ECUT).

Implementation of the "HOWFARLESS" option has necessitated changes in the HOWFAR and HOWNEAR subroutines so that, when the option is used, voxel boundaries within the phantom are ignored, and distances (perpendicular and along the particle trajectory) are calculated with respect to the extreme outer boundaries of the phantom.

\subsubsection{Charged particle transport}

All of the logic described in this section has been implemented in DOSXYZnrc's AUSGAB (scoring) subroutine.

1. If the particle is about to take a step and energy will be deposited over the step, the perpendicular distance to the nearest voxel boundary is calculated and compared to the straight-line distance of the proposed step (VSTEP).

2. If VSTEP is less than the perpendicular distance to the nearest voxel boundary, then the particle's step will not take it out of the current voxel, and normal transport occurs, with all of the energy deposited in the current voxel.

3. If VSTEP is greater than the perpendicular distance to the nearest voxel boundary, then the particle could step across the voxel boundary. The particle's initial position, $\left(x_{i}, y_{i}, z_{i}\right)$, and direction, $\left(u_{i}, v_{i}, w_{i}\right)$, are saved along with the energy that will be deposited over the step. The particle is then allowed to take the step, unrestricted by voxel boundaries.

4. After the unrestricted step, a check is done on the particle's final position, $\left(x_{f}, y_{f}, z_{f}\right)$, to determine if it finishes the step in a different voxel than it started in (having VSTEP greater than the perpendicular distance to the nearest voxel boundary does not guarantee that it will leave the voxel). If not, then the energy deposited over the step is simply dumped in the voxel. Note that this saves further transport time but assumes that the particle does not exit and then reenter the voxel. It 
will be shown in Section 3.5.1.2 below that the inaccuracies inherent in such an assumption are insignificant.

5. If the particle did leave the voxel, then the unrestricted step is approximated using either a forwardhinged step (calculated using equations (3.2) and (3.4) above) or a reverse-hinged step (calculated using equations (3.5) and (3.6) above). The selection of which approximation to use is done by comparing a random number (range $[0,1]$ ) to a threshold value. The threshold value determines the ratio of forward-hinged:reverse-hinged steps over the entire calculation. Note that if the step was taken in single-scattering mode (i.e. not a condensed history step) then the path is not curved, $t_{1}$ is set equal to the step length, and $t_{2}$ is set to 0 .

6. For each voxel $k$ traversed by the hinged step, the dose deposited in the voxel, $D_{k}$, is calculated using:

$$
D_{k}=\frac{t_{k}}{t_{v}} \Delta E
$$

where $t_{k}$ is the distance traveled in the voxel and $\Delta E$ is the energy deposited over the entire step (pathlength $=t_{v}$ ). Equation (3.7) assumes that energy is deposited evenly over the entire path. This approximation is valid when the restricted stopping power changes slowly with energy, a condition typically satisfied over the energy range that is of interest for beam commissioning.

\subsubsection{Minimum step length}

When the "HOWFARLESS" algorithm is used, all charged particle steps are restricted to being greater than or equal to half the minimum distance between voxel boundaries provided that this distance is not greater than the maximum allowable electron step length (user input Smax [6]). This restriction overrides any limitation on step length due to the maximum allowable energy loss/step (user input estepe[6]).

The minimum step length significantly reduces the CPU time by reducing the total number of steps that must be tracked. It is shown in Section 3.5.1.2 below that any approximations introduced by enforcing a minimum step length do not have a significant impact on the accuracy of "HOWFARLESS" calculations.

\subsubsection{Range rejection}

Charged particle range rejection in DOSXYZnrc relies on having an accurate calculation of the perpendicular distance to the nearest voxel boundary. The range that a particle can travel before reaching the transport cutoff energy (ECUT) is then compared to this distance and, if it is less, particle transport is immediately terminated and the total energy is deposited in the current voxel. Normally, the perpendicular distance to the nearest voxel boundary is provided by DOSXYZnrc's HOWNEAR subroutine, and the actual termination of the particle is taken care of by EGSnrc[6]. However, when the "HOWFARLESS" option is used, HOWNEAR calculates the perpendicular distance to the nearest phantom boundary (see Section 3.3.1 above). If this distance were used for range rejection, charged particles would be terminated too early, resulting in dose inaccuracies. To circumvent this problem, range rejection (if turned on) is 
incorporated into the "HOWFARLESS" transport logic by comparing the range of the charged particle to the perpendicular distance to the nearest voxel boundary calculated in step 1 of the "HOWFARLESS" algorithm (see Section 3.3.2 above).

\subsubsection{Photon transport}

Photon transport when the "HOWFARLESS" option is used is similar to standard transport with HOWFAR, except that photon steps are not halted at voxel boundaries. Since photons do not deposit energy during a step, there is no need to retrace steps to determine which voxels were traversed. It also means that information about the current region/voxel of a photon is lost. This information is recovered immediately prior to a Compton, photoelectric, pair production or Rayleigh event, so that it can be passed on to the secondary charged particle(s).

\subsection{DOSXYZnrc phantom calculations}

This section describes the DOSXYZnrc phantom simulations used to determine the accuracy and efficiency of the "HOWFARLESS" option.

\subsubsection{Accuracy calculations}

In order to determine the accuracy of the "HOWFARLESS" algorithm, it is important to compare its results to those with HOWFAR under conditions where charged particle equilibrium does not hold. To this end, DOSXYZnrc simulations are performed in a water phantom with a small incident beam field $\left(0.3 \times 0.3 \mathrm{~cm}^{2}\right)$ centered on a column of voxels with dimensions $0.25 \times 0.25 \times 0.25 \mathrm{~cm}^{3}$. The beams studied are 6 and $20 \mathrm{MeV}$ monoenergetic electron beams, and 6 and $24 \mathrm{MV}$ photon beams with energies defined using the spectrum determined by Mohan et al[10] for Clinac beams with these energies. Phantom $\mathrm{X} \times \mathrm{Y} \times \mathrm{Z}$ (where $\mathrm{Z}$ is depth) dimensions are $60.25 \times 60.25 \times 5 \mathrm{~cm}^{3}$ for the $6 \mathrm{MeV}$ electron beam, $60.25 \times 60.25 \times 30 \mathrm{~cm}^{3}$ for the $20 \mathrm{MeV}$ electron beam, $120.25 \times 120.25 \times 30 \mathrm{~cm}^{3}$ for the $6 \mathrm{MV}$ photon beam, and $120.25 \times 120.25 \times 60 \mathrm{~cm}^{3}$ for the $24 \mathrm{MV}$ photon beam. Note that the $0.25 \times 0.25 \times 0.25 \mathrm{~cm}^{3}$ voxels are only in a column down the central axis, with the remainder of the phantom being defined by very large voxels. Electron and photon transport cutoff energies (ECUT and PCUT) in these simulations are $0.7 \mathrm{MeV}$ and $0.01 \mathrm{MeV}$, respectively. Simulations are done with and without range rejection. If range rejection is used, then ESAVE (the energy below which range rejection is considered) is set to $2 \mathrm{MeV}$ for the $6 \mathrm{MeV}$ electron and $6 \mathrm{MV}$ photon beams and $5 \mathrm{MeV}$ for the $20 \mathrm{MeV}$ electron and $24 \mathrm{MV}$ photon beams.

Calculations using HOWFAR and "HOWFARLESS" are compared by calculating the root mean square difference between central-axis doses over a range of depths ( $Z$ values). For voxels going from depth $Z_{\text {min }}$ to $Z_{\text {max }}$, the root mean square difference, RMSD, is given by:

$$
\mathrm{RMSD}=\sqrt{\frac{1}{N_{\text {vox }}} \sum_{i=Z_{\min }}^{Z_{\max }} \frac{\left(D_{i h w f l}-D_{i h w f}\right)^{2}}{\left(\sigma_{i h w f l}^{2}+\sigma_{i h w f}^{2}\right)}}
$$


where $N_{v o x}$ is the number of voxels included between $Z_{\text {min }}$ and $Z_{\text {max }}, D_{i h w f l}$ and $\sigma_{i h w f l}$ are the dose and uncertainty in voxel $i$ with "HOWFARLESS", and $D_{i h w f}$ and $\sigma_{i} h w f$ are the dose and uncertainty in voxel $i$ with HOWFAR. The relationship between RMSD and the average systematic bias, $\langle B\rangle$, between doses calculated with "HOWFARLESS" and HOWFAR is given by:

$$
\langle B\rangle \approx \sqrt{\left(\left\langle\sigma_{h w f l}\right\rangle^{2}+\left\langle\sigma_{h w f}\right\rangle^{2}\right)\left(R M S D^{2}-1\right)}
$$

An RMSD close to unity indicates that there is no systematic bias introduced by the "HOWFARLESS" method. The $Z$ range of voxels included in the analysis depends on beam energy and type: $Z=0-1.75 \mathrm{~cm}$ (7 voxels) for the $6 \mathrm{MeV}$ electron beam, $\mathrm{Z}=0-3.5 \mathrm{~cm}$ (14 voxels) for the $20 \mathrm{MeV}$ electron beam, $\mathrm{Z}=0-25$ $\mathrm{cm}$ (100 voxels) for the $6 \mathrm{MV}$ photon beam, and $\mathrm{Z}=0-30 \mathrm{~cm}$ (120 voxels) for the $24 \mathrm{MV}$ photon beam. In all cases, the $\mathrm{Z}$ range includes $D_{\max }$ (depth of maximum dose) and $D_{50}$ (depth at $50 \%$ of maximum dose) and all doses have uncertainty less than $0.1 \%$

In order to be able to obtain an uncertainty estimate on RMSD, all calculations are split into 15 separate jobs (with equal number of histories). The RMSD is then calculated between corresponding pairs of HOWFAR and "HOWFARLESS" jobs and uncertainty is estimated as the standard deviation in the resulting 15 values of RMSD. All jobs are run on $1.8 \mathrm{GHz}$ Opteron 244 CPU's.

"HOWFARLESS" calculations are performed over a range of maximum allowable charged particle step lengths (input variable Smax). For the $6 \mathrm{MeV}$ electron and $6 \mathrm{MV}$ photon beams, Smax is varied over the range $0.1-2 \mathrm{~cm}$, while for the $20 \mathrm{MeV}$ electron and $24 \mathrm{MV}$ photon beams, values of Smax in the range $0.1-5 \mathrm{~cm}$ are examined. At the upper end of these ranges maximum step length is no longer limited by Smax but by the maximum fractional energy loss/step (input estepe). Note that "HOWFARLESS" calculations with small values of Smax are expected to agree with standard HOWFAR calculations, but the potential efficiency increases of the "HOWFARLESS" option are only realized for larger values of Smax. Ideally, Smax becomes an unnecessary limit, and "HOWFARLESS" calculations are accurate where estepe becomes the only limiting factor. Calculations with HOWFAR all use the default Smax of $1 \times 10^{10}$ $\mathrm{cm}$. See [6] for a more general discussion of the parameters Smax and estepe.

For each value of Smax, the effect on accuracy of the mixture of forward- and reverse-hinged step approximations (see Section 3.2 above) is investigated by doing "HOWFARLESS" calculations with forwardhinged:reverse-hinged ratios of 1:0 (all steps forward-hinged), 2:1, 1:1, 0:1 (all steps reverse-hinged).

In the case of the $24 \mathrm{MV}$ and $6 \mathrm{MV}$ photon beams, accuracy calculations are performed with $40 \times$ photon splitting. Although this splitting number does not result in peak efficiency for these sources, in these accuracy checks it is sufficient to show that the photon splitting algorithm and the "HOWFARLESS" algorithm can be used together.

All HOWFAR and "HOWFARLESS" accuracy calculations use the exact (single-scattering) boundary crossing algorithm (BCA) instead of the faster PRESTA-I BCA (the default in DOSXYZnrc). It will be shown in Section 3.5.1.1 below that PRESTA-I boundary crossing results in inaccuracies when charged particle equilibrium does not hold. For more information about these boundary crossing algorithms, please see [6]. 


\subsubsection{Efficiency calculations}

In contrast to the accuracy calculations, the efficiency calculations use a DOSXYZnrc water phantom divided up into uniform voxels. Incident beams investigated are: 6 and $20 \mathrm{MeV}$ monoenergetic electron beams with $10 \times 10 \mathrm{~cm}^{2}$ fields; 6 and $24 \mathrm{MV}$ photon beams with $10 \times 10 \mathrm{~cm}^{2}$ fields using Clinac energy spectra for these energies[10] (called "simple photon beams" to distinguish them from the photon beams from BEAMnrc-simulated accelerators); $10 \times 10 \mathrm{~cm}^{2}$ and $40 \times 40 \mathrm{~cm}^{2} 6 \mathrm{MV}$ photon beams from a BEAMnrc-simulated Elekta SL25 accelerator; $10 \times 10 \mathrm{~cm}^{2}$ and $40 \times 40 \mathrm{~cm}^{2} 18 \mathrm{MV}$ photon beams from a BEAMnrc-simulated Siemens KD2 accelerator. In the case of the simulated accelerators, both phase space sources and full BEAMnrc simulation sources are investigated, with the exception of the $40 \times 40$ $\mathrm{cm}^{2} 18 \mathrm{MV}$ beam, for which only a BEAMnrc simulation source is studied. See Section 3.4.2.1 below for more information about the BEAMnrc simulations.

The overall phantom $\mathrm{X} \times \mathrm{Y} \times \mathrm{Z}$ dimensions are $20.5 \times 20.5 \times 30 \mathrm{~cm}^{3}$ for the $10 \times 10 \mathrm{~cm}^{2}$ beams and $50 \times 50 \times 30$ $\mathrm{cm}^{3}$ for the $40 \times 40 \mathrm{~cm}^{2}$ beams. The $20.5 \times 20.5 \times 30 \mathrm{~cm}^{3}$ phantoms are divided into $0.5 \times 0.5 \times 0.5 \mathrm{~cm}^{3}$ voxels, and the $50 \times 50 \times 30 \mathrm{~cm}^{3}$ phantoms are divided into $1 \times 1 \times 1 \mathrm{~cm}^{3}$ voxels. This voxel resolution is sufficient for most beam commissioning calculations. For the phase space and BEAMnrc simulation sources, the DOSXYZnrc calculation also includes $\sim 50 \mathrm{~cm}$ of air between the bottom of the field-defining jaws and the top of the phantom as a single region (see Section 3.4.2.1 below for more details). Transport in the phantom is done with an electron transport cutoff (ECUT) of $0.7 \mathrm{MeV}$ and photon transport cutoff (PCUT) of $0.01 \mathrm{MeV}$. Charged particle range rejection is used in all simulations with an ESAVE (energy below which range rejection is considered) equal to $2 \mathrm{MeV}$ for the $6 \mathrm{MV}$ photon and $6 \mathrm{MeV}$ electron beams and ESAVE $=5 \mathrm{MeV}$ for the $20 \mathrm{MeV}$ electron beam and 18 and $24 \mathrm{MV}$ photon beams.

Phantom calculations are done with both standard HOWFAR and using the "HOWFARLESS" option and the efficiencies using the two methods are compared. The efficiency of a calculation, $\varepsilon$, is defined as:

$$
\varepsilon=\frac{1}{s^{2} T}
$$

where $s$ is the uncertainty on the quantity of interest and $T$ is the CPU time required to achieve this uncertainty. In this study, $s^{2}$ is the sum of the squares of the fractional uncertainties in all doses greater than $50 \%$ of the maximum dose $D_{\max }$, divided by the number of voxels, $N_{v o x}$, included:

$$
s^{2}=\frac{1}{N_{\text {vox }}} \sum_{D_{i} \geq D_{\max } / 2}\left(\frac{\Delta D_{i}}{D_{i}}\right)^{2}
$$

This definition of efficiency was proposed by Rogers and Mohan[19] for benchmarking clinical Monte Carlo codes. In the case of phase space sources from the BEAMnrc-simulated 6 and $18 \mathrm{MV}$ accelerators, the time required in the second-stage BEAMnrc simulation (i.e. transport particles through the fielddefining jaws) to generate the source particles is calculated and added to the CPU time, $T$. In the case of full BEAMnrc simulation sources, $T$ implicitly includes the time required for transport through the entire treatment head to obtain the necessary source particles in DOSXYZnrc.

To investigate the effects of photon splitting on efficiency of photon beam dose calculations (i.e. for 
the simple 6 and $24 \mathrm{MV}$ photon beams and for the BEAMnrc-simulated 6 and $18 \mathrm{MV}$ accelerators), the photon splitting number, $\mathrm{n}_{-}$split, is varied from 1 (no splitting) to 100 in both HOWFAR and "HOWFARLESS" calculations. In the case of the 6 and $18 \mathrm{MV}$ accelerators, contaminant electrons in the beam are also split by $\mathrm{n}_{-}$split as soon as they enter the phantom. This ensures that all particles contributing to the dose are of the same weight (higher-weight particles can compromise statistics).

Efficiency calculations are done using both the exact BCA (the BCA used in the accuracy calculations) and the default PRESTA-I BCA. Although the PRESTA-I BCA is shown to result in dose inaccuracies when charged particle equilibrium does not hold or when the phantom is not divided into uniform voxels throughout (see Section 3.5.1.1 below), it is up to 4 times more efficient than the exact BCA and is suitable for most beam commissioning calculations where beam fields are generally large (i.e. charged particle equilibrium exists) and phantoms are usually divided up into uniform voxels. All efficiency calculations use the default value for the maximum allowed charged particle step length (input variable Smax), which is $1 \times 10^{10} \mathrm{~cm}$ with the exact BCA and $5 \mathrm{~cm}$ with the PRESTA-I BCA. In the case of "HOWFARLESS" calculations with the $6 \mathrm{MeV}$ and $20 \mathrm{MeV}$ monoenergetic electron beams, efficiencies are also evaluated for values of Smax over the range $0.01-5 \mathrm{~cm}$ (exact BCA) to examine how the efficiency varies as a function of the maximum allowable step length.

All "HOWFARLESS" efficiency calculations have a ratio of forward-hinged:reverse-hinged step approximations of 1:1. It will be seen in the Section 3.5.1.2 below, that this ratio results in the greatest accuracy over all values of Smax for all beam types.

\subsubsection{BEAMnrc simulations}

The BEAMnrc simulations of the 6 MV SL25 accelerator and the 18 MV KD2 accelerator in these efficiency calculations are identical to those used by Kawrakow and Walters [8] in their paper on DOSXYZnrc efficiency and BEAMnrc simulation sources. As mentioned in Section 3.4.2 above, phase space sources and BEAMnrc simulation sources are investigated. BEAMnrc simulations to generate phase space sources are done in two stages in which a phase space source collected immediately above the field-defining jaws (stage one) is used as a source to generate a phase space file immediately below the jaws (stage two) for use in the DOSXYZnrc calculations. This two-stage method is commonly used to allow one to generate phase space files for different jaw settings without having to re-simulate the upper portion of the accelerator. BEAMnrc simulation sources are done in one stage with source data collected immediately below the field-defining jaws. Directional bremsstrahlung splitting (DBS) is used to optimize BEAMnrc simulations in all cases. The air between the jaws and the phantom (at SSD=100 cm) is always included in the DOSXYZnrc simulations as this has been shown[8] to be more efficient. 


\subsection{Results}

\subsubsection{Accuracy}

\subsubsection{PRESTA-I versus exact boundary crossing algorithms (BCAs)}

Figure 3.2 shows differences between central-axis doses in a water phantom calculated using the exact BCA and PRESTA-I BCA for the $6 \mathrm{MV}$ photon beam (with the Clinac energy spectrum of Mohan et al[10]). Differences are shown as a percentage of the local dose computed with the exact BCA. Figure 3.2a shows differences for a beam field of $0.3 \times 0.3 \mathrm{~cm}^{2}$ and central axis voxel $\mathrm{X} \times \mathrm{Y} \times \mathrm{Z}$ dimensions of $0.25 \times 0.25 \times 0.25 \mathrm{~cm}^{3}$. This beam/voxel size geometry is the same as that used for comparing "HOWFARLESS" and HOWFAR results in the accuracy studies discussed in Section 3.5.1.2 below and is a case where charged particle equilibrium does not hold. Figures $3.2 \mathrm{~b}$ and $3.2 \mathrm{c}$ show differences for a $10 \times 10 \mathrm{~cm}^{2}$ beam field with central axis voxel dimensions of $0.25 \times 0.25 \times 0.25 \mathrm{~cm}^{3}$ and $1 \times 1 \times 0.25 \mathrm{~cm}^{3}$. Note that the overall phantom $\mathrm{X} \times \mathrm{Y} \times \mathrm{Z}$ dimensions of $120.5 \times 120.5 \times 30 \mathrm{~cm}^{3}$ are filled in using voxels with large $\mathrm{X}$ and $\mathrm{Y}$ dimensions beyond the central axis.

Figure 3.2 demonstrates that the use of PRESTA-I boundary crossing in a HOWFAR calculation can result in a significant overestimate of dose. When charged particle equilibrium does not hold (Figure $3.2 \mathrm{a}$ ), the overestimate can be by as much as $2 \%$. The overestimate decreases to $0.5 \%$ under conditions of charged particle equilibrium with the same voxel size (Figure $3.2 \mathrm{~b}$ ), and becomes insignificant under conditions of charged particle equilibrium when the voxels are enlarged to $1 \times 1 \times 0.25 \mathrm{~cm}^{3}$. This behavior has been documented in more detail by Walters and Kawrakow[20] who found that the use of PRESTA-I boundary crossing can result in dose overestimates by as much as $2.5 \%$ when charged particle equilibrium does not hold or when the phantom is not divided into the voxels of the same size throughout.

When the "HOWFARLESS" option is used, the difference between doses calculated with the PRESTAI BCA and exact BCA is insignificant in all cases (Figures 3.2a-c). This indicates that the use of the PRESTA-I BCA at the upper boundary of the phantom (the only boundary in this calculation where use of the BCA could possibly have an effect on the central-axis dose) does not result in a significant over-prediction of the dose.

In all of the accuracy calculations described below charged particle equilibrium does not hold $(0.3 \times 0.3$ $\mathrm{cm}^{2}$ beams on $0.25 \times 0.25 \times 0.25 \mathrm{~cm}^{3}$ voxels only down the central axis). Therefore, a meaningful comparison between doses calculated with HOWFAR and using the "HOWFARLESS" option is only possible with exact boundary crossing. A further conclusion from the above results is that, if PRESTA-I boundary crossing is used, doses calculated with the "HOWFARLESS" option will be more accurate (i.e. closer to those calculated using exact boundary crossing) over a broader range of beam and phantom geometries than doses calculated with HOWFAR.

Most beam commissioning calculations are done with coarser voxel resolution $\left(0.5 \times 0.5 \times 0.5 \mathrm{~cm}^{3}\right.$ voxels or larger) and under conditions of charged particle equilibrium and with the phantom divided into voxels of the same size throughout, so PRESTA-I boundary crossing may be used to calculate dose. This is desirable, since it is a faster boundary crossing algorithm and significantly decreases the CPU 


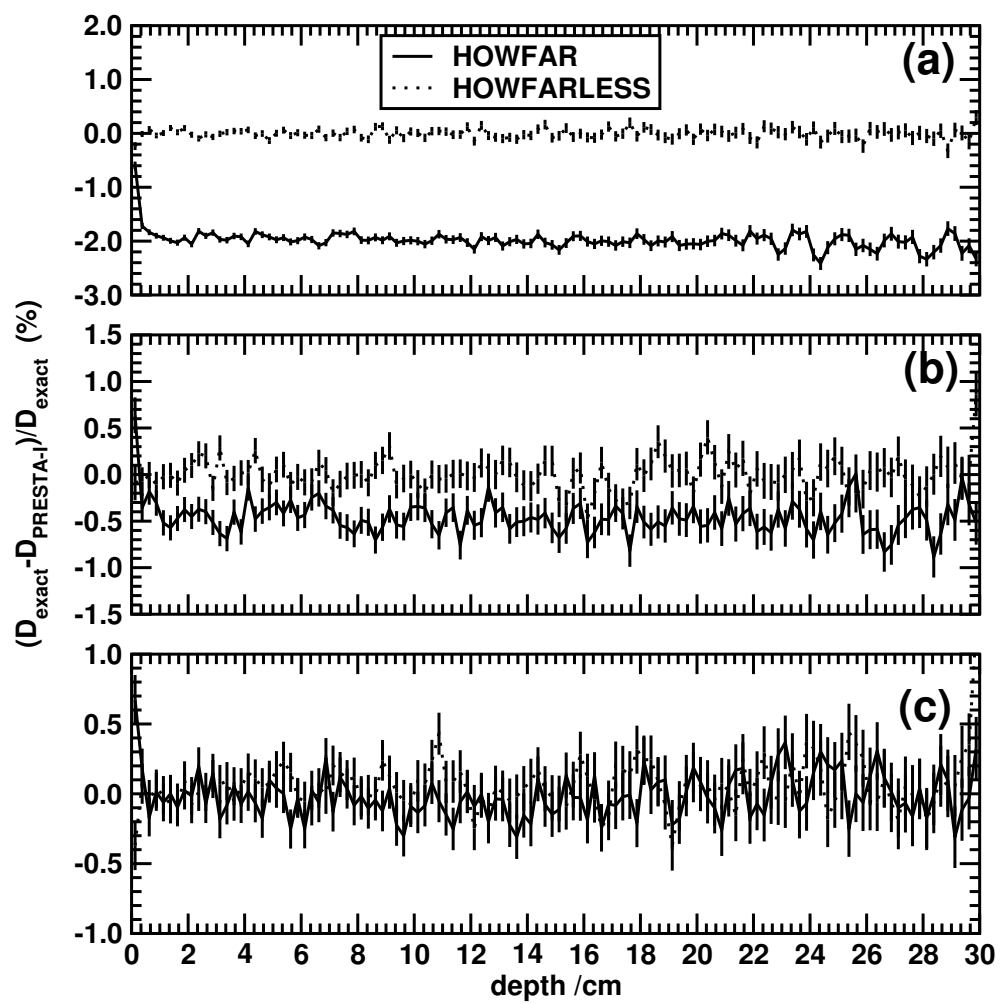

Figure 3.2: Difference between central axis doses calculated using exact and PRESTA-I boundary crossing algorithms vs depth in the water phantom for a $6 \mathrm{MV}$ photon beam with (a) a $0.3 \times 0.3 \mathrm{~cm}^{2}$ field and central-axis voxel $\mathrm{X} \times \mathrm{Y} \times \mathrm{Z}$ dimensions of $0.25 \times 0.25 \times 0.25 \mathrm{~cm}^{3}$ (b) a $10 \times 10 \mathrm{~cm}^{2}$ field and $0.25 \times 0.25 \times 0.25$ $\mathrm{cm}^{3}$ central-axis voxels and (c) a $10 \times 10 \mathrm{~cm}^{2}$ field and $1 \times 1 \times 0.25 \mathrm{~cm}^{3}$ central-axis voxels. The photon beam has the Clinac energy spectrum determined by Mohan et al[10]. Differences are shown as a percentage of the dose with the exact BCA $\left(D_{\text {exact }}\right)$ for calculations using HOWFAR and "HOWFARLESS".

time for a simulation, especially when using standard HOWFAR. The impact of the boundary crossing algorithm on the efficiencies of calculations done using HOWFAR and with the "HOWFARLESS" option will be discussed in Section 3.5.2.2 below.

\subsubsection{Comparison of HOWFAR with "HOWFARLESS"}

Figures 3.3 and 3.4 show the root mean square difference, RMSD (calculated using Equation (3.8)), between central-axis doses calculated with HOWFAR and "HOWFARLESS" as a function of the maximum allowed charged particle step length (Smax) in the "HOWFARLESS" calculation. Results are shown for the $6 \mathrm{MeV}$ monoenergetic electron beam (Figure 3.3a), the $6 \mathrm{MV}$ photon beam with Clinac spectrum (Figure 3.3b), the $20 \mathrm{MeV}$ monoenergetic electron beam (Figure 3.4a), and the $24 \mathrm{MV}$ photon beam with 
Clinac spectrum (Figure $3.4 \mathrm{~b}$ ). Beam fields are all $0.3 \times 0.3 \mathrm{~cm}^{2}$ and central axis voxels are $0.25 \times 0.25 \times 0.25$ $\mathrm{cm}^{3}$, thus setting up a condition where charged particle equilibrium does not hold. For each beam, the RMSD is evaluated over a range of phantom depths indicated in the figure captions. Results are shown for "HOWFARLESS" calculations with ratios of forward-hinged:reverse-hinged step approximations of 1:0 (all forward-hinged), 0:1 (all reverse hinged), 2:1 and 1:1. The RMSD for "HOWFARLESS" calculations in which the dose deposition step has no hinge is also shown for the $6 \mathrm{MeV}$ electron and $6 \mathrm{MV}$ photon beams (Figures 3.3a and 3.3b, labeled dotted lines) for comparison.
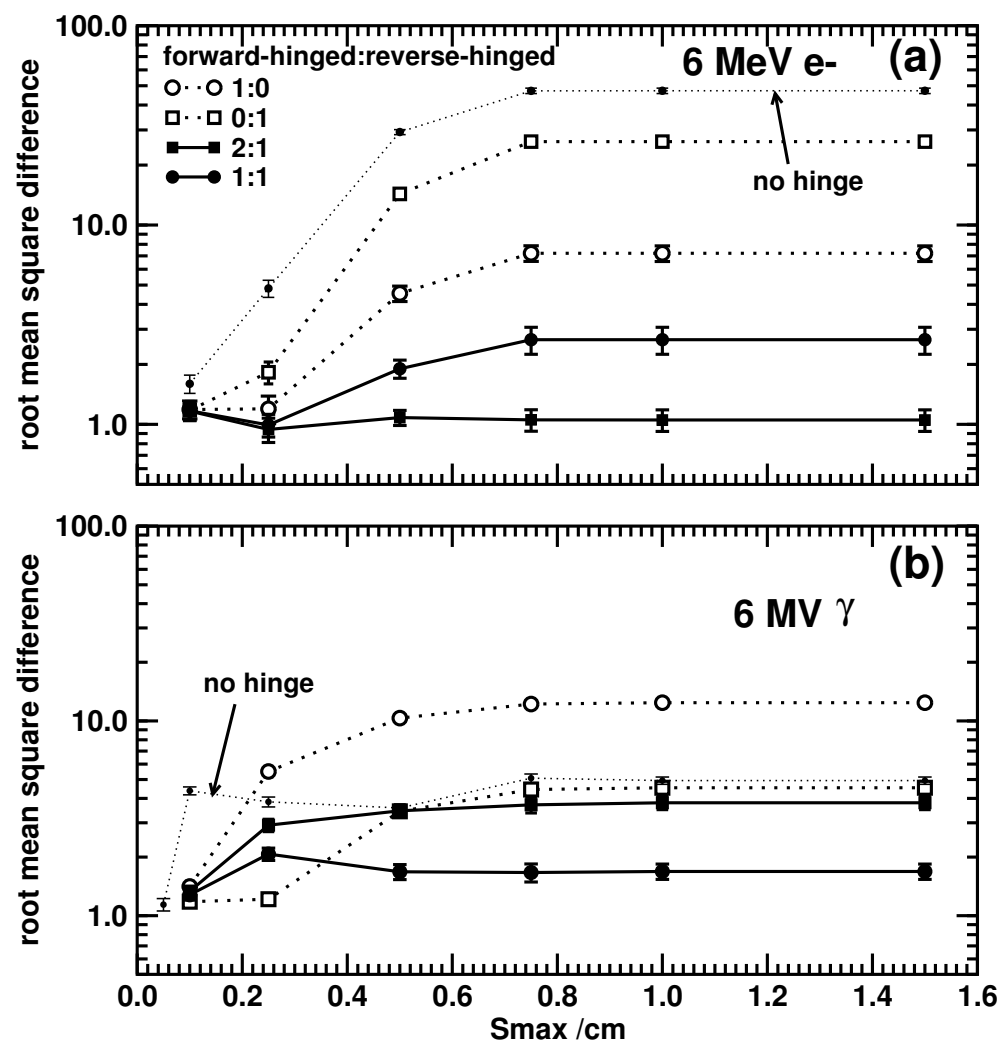

Figure 3.3: Root mean square difference (RMSD) between doses in water calculated with HOWFAR and "HOWFARLESS" as a function of the maximum allowed charged particle step length (Smax) in the "HOWFARLESS" calculation for (a) a $6 \mathrm{MeV}$ monoenergetic electron beam and (b) a $6 \mathrm{MV}$ photon beam using the Clinac spectrum. The RMSD is shown on a log scale to emphasize differences over the entire range of values. Incident beams are $0.3 \times 0.3 \mathrm{~cm}^{2}$ and dose is calculated in $0.25 \times 0.25 \times 0.25 \mathrm{~cm}^{3}$ voxels on the central axis. The root mean square difference between doses is determined using Equation (3.8) and is evaluated over phantom depths of (a) $0-1.75 \mathrm{~cm}$ and (b) $0-25 \mathrm{~cm}$. Results are shown for "HOWFARLESS" calculations with ratios of forward-hinged:reverse-hinged step approximations of 1:0 (all forward-hinged), 2:1, 1:1 and 0:1 (all reverse-hinged). Additional results are shown for "HOWFARLESS" results with no hinge in the step (labeled dotted lines). 

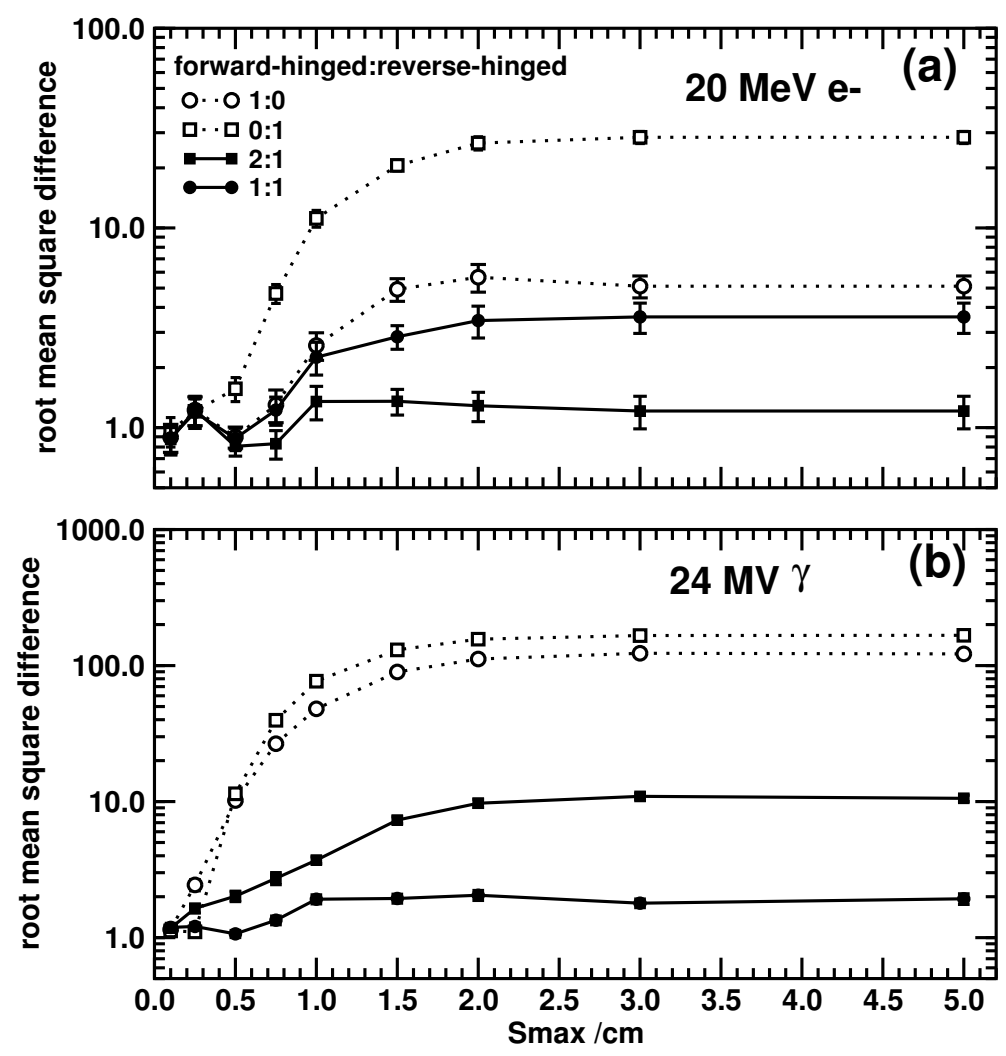

Figure 3.4: Root mean square difference (RMSD) between doses in water calculated with HOWFAR and "HOWFARLESS" as a function of the maximum allowed charged particle step length (Smax) in the "HOWFARLESS" calculation for (a) a $20 \mathrm{MeV}$ monoenergetic electron beam and (b) a $24 \mathrm{MV}$ photon beam using the Clinac spectrum. Beam and voxel sizes are the same as in Figure 3.3. Root mean square difference is evaluated over phantom depths of (a) $0-3.5 \mathrm{~cm}$ and (b) $0-30 \mathrm{~cm}$. Results are shown for "HOWFARLESS" calculations with forward-hinged:reverse-hinged ratios of 1:0 (all forward-hinged), 2:1, 1:1 and 0:1 (all reverse-hinged).

As expected, "HOWFARLESS" results are in good agreement (RMSD 1) with those calculated using standard HOWFAR when Smax is small $(\leq 0.25 \mathrm{~cm}$ for the $6 \mathrm{MeV}$ electron and $6 \mathrm{MV}$ photon beam, $\leq 0.5$ $\mathrm{cm}$ for the $20 \mathrm{MeV}$ electron and $24 \mathrm{MV}$ photon beam). As Smax in the "HOWFARLESS" calculations is increased, however, the calculations using only forward-hinged (1:0) or reverse-hinged (0:1) curved step approximations deviate significantly from the HOWFAR results. In all cases, a value of Smax is reached beyond which there are no significant changes in root mean square difference. This value $(0.75 \mathrm{~cm}$ in the $6 \mathrm{MV}$ photon and $6 \mathrm{MeV}$ electron beams, $3 \mathrm{~cm}$ in the $24 \mathrm{MV}$ photon and $20 \mathrm{MeV}$ electron beams) is the point at which maximum step length is no longer limited by Smax but by the maximum allowed fractional energy loss/step (EGSnrc input variable estepe), which is set to a default value of 0.25 in 


\section{DOSXYZnrc.}

In general, in order to obtain acceptable accuracy over all values of Smax using the "HOWFARLESS" option it is necessary to use a mixture of forward-hinged and reverse-hinged step approximations. Figures 3.3a and 3.4a show that, for monoenergetic electron beams, a forward-hinged:reverse-hinged ratio of 2:1 results in better agreement with HOWFAR results over all values of Smax than a ratio of 1:1. Figures $3.3 \mathrm{~b}$ and $3.4 \mathrm{~b}$, however, indicate that, for photon beams, a 1:1 ratio is preferable. Ultimately, the 1:1 ratio is chosen as the default for "HOWFARLESS" simulations for several reasons: 1) It is more accurate for photon beams, and beam commissioning calculations (where the "HOWFARLESS" option is likely to be most useful) for monoenergetic electron beams are rare. 2) In the case of the $24 \mathrm{MV}$ photon beam the 2:1 ratio results in unacceptable inaccuracies. 3) Even for monoenergetic electron beams the 1:1 ratio results in a maximum RMSD of $\sim 4$. Given the uncertainty on the doses used in this comparison $(\leq 0.1 \%)$, $\mathrm{RMSD}=4$ corresponds to a systematic bias of $0.5 \%$ or less (see Equation (3.9), which is an acceptable level of accuracy for practical beam commissioning calculations.

If the hinge is omitted entirely (i.e. dose deposited in a straight-line from the particle's initial position to its final position), as shown in Figures 3.3a and 3.3b, then results with "HOWFARLESS" deviate significantly from those with HOWFAR even for small values of Smax $(0.1 \mathrm{~cm}$ in the case of the $6 \mathrm{MV}$ photon and $0.25 \mathrm{~cm}$ in the case of the $6 \mathrm{MeV}$ electron beam). These results underscore the importance of using a mixture of hinged steps and indicate the limitations of some other published methods for accelerating dose calculations, such as DOSSCORE[21] and MCDOSE[22], that also allow charged particle steps across multiple voxel boundaries but assume dose deposition only along the straight-line path.

Figure 3.5a shows depth-dose curves calculated with HOWFAR and using "HOWFARLESS" in the $24 \mathrm{MV}$ photon beam with the geometry identical to that used for the accuracy calculations described above. The "HOWFARLESS" calculation uses a 1:1 ratio of forward-hinged:reverse-hinged step approximations and has Smax set to the default value of $1 \times 10^{10} \mathrm{~cm}$ (exact BCA). Figure $3.5 \mathrm{~b}$ shows the percentage difference between the doses and confirms that the doses agree within uncertainty.

\subsubsection{Efficiency}

\subsubsection{Efficiency vs. Smax}

Figure 3.6 presents the efficiency defined in Eq. (3.10) as a function of Smax (the maximum allowed electron step length) for $6 \mathrm{MeV}$ and $20 \mathrm{MeV}$ monoenergetic electron beams with a $10 \times 10 \mathrm{~cm}^{2}$ field. The values at which maximum efficiency are reached are those where the maximum step length is no longer limited by Smax but by the maximum allowable fractional energy loss/step, estepe (see Section 3.5.1.2 above), which is set to a default of 0.25 in DOSXYZnrc. For $6 \mathrm{MeV}$ and $20 \mathrm{MeV}$ electrons, this limitation results in a maximum step lengths of $0.75 \mathrm{~cm}$ and $2.4 \mathrm{~cm}$ respectively. Figure 3.6 reinforces the importance of setting Smax to a value at least as large as that dictated by estepe to maximize the efficiency of the "HOWFARLESS" option (the defaults of $1 \times 10^{10} \mathrm{~cm}$ for the exact BCA and $5 \mathrm{~cm}$ for the PRESTA-I BCA are sufficient for most beams). The figure also points to the possibility of increasing estepe as a means of increasing "HOWFARLESS" efficiency (but this possibility is not pursued in this investigation). 


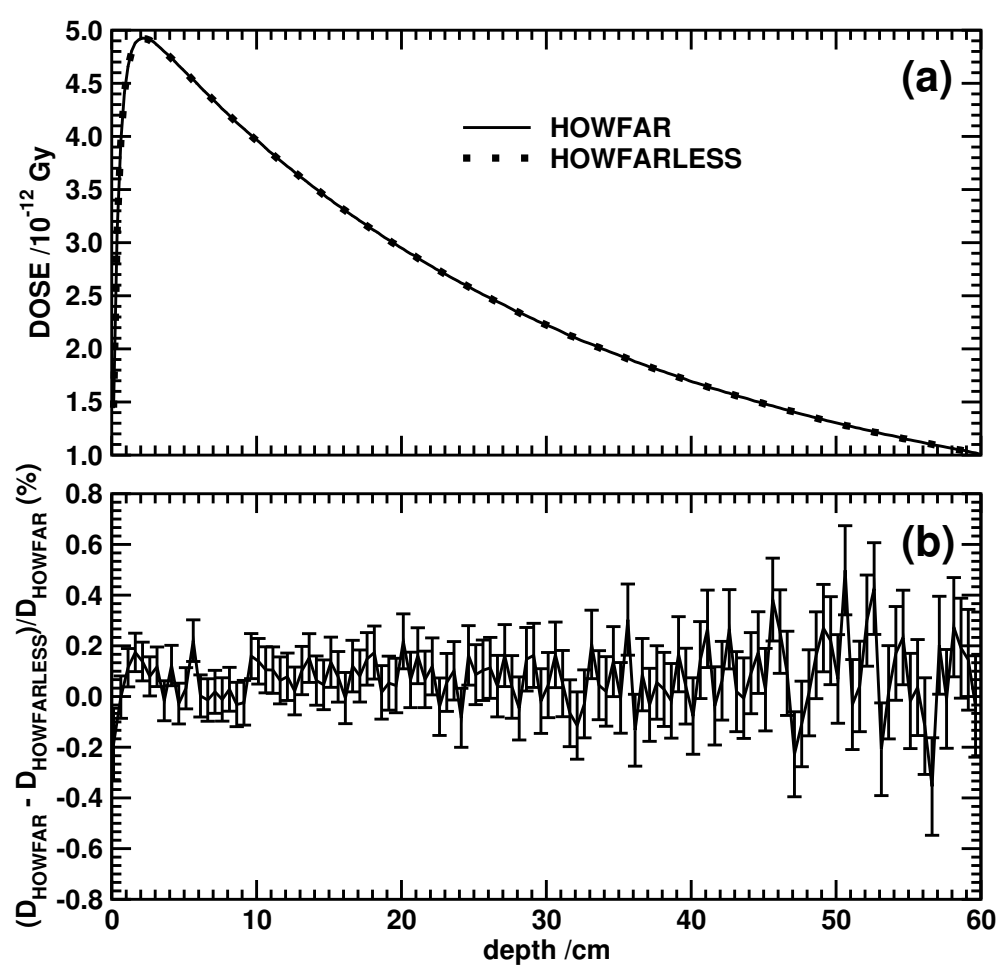

Figure 3.5: Central-axis depth dose curves (a) and the difference between HOWFAR and "HOWFARLESS" doses as a percentage of the HOWFAR dose (b) for the 24 MV photon beam with Clinac energy spectrum. Simulation geometry is identical to that used to determine the root mean square difference in Figure $3.4 \mathrm{~b}$.

\subsubsection{Comparison with HOWFAR}

Figure 3.7 shows the efficiency defined in Eq. (3.10) in a DOSXYZnrc water phantom (voxel dimensions $0.5 \times 0.5 \times 0.5 \mathrm{~cm}^{3}$ ) as a function of the photon splitting number (n_split) for the $10 \times 10 \mathrm{~cm}^{2} 6 \mathrm{MV}$ photon beam from a simulated Elekta SL25 accelerator. Solid lines depict results using the "HOWFARLESS" algorithm, and dashed lines correspond to results obtained with HOWFAR. When the exact boundary crossing algorithm is used (Figure 3.7a), the peak efficiency using "HOWFARLESS" is a factor of $\sim 4$ higher than with HOWFAR when a phase space source is used (circles) and a factor of $\sim 3.5$ higher when a BEAMnrc simulation source is used (squares). Note that the use of "HOWFARLESS" shifts the peak efficiency to higher values of n_split (from 24 with HOWFAR to 40 with "HOWFARLESS" in the case of the phase space source and from 32 with HOWFAR to 50 with "HOWFARLESS" in the case of the BEAMnrc simulation source). The relative increase in peak efficiency with the "HOWFARLESS" method is smaller for the BEAMnrc simulation source because, as the DOSXYZnrc phantom simulation becomes more efficient, the time required to generate source particles plays a more significant role in efficiency. 


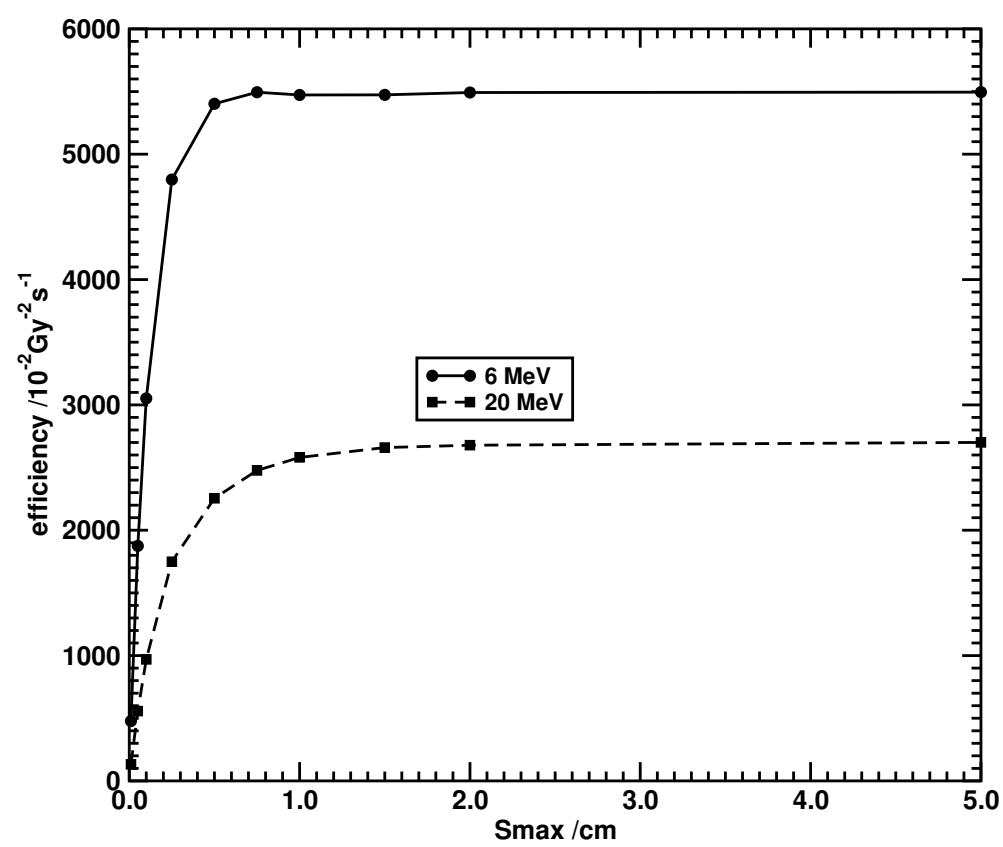

Figure 3.6: Efficiency of all doses $>0.5 \mathrm{D}_{\max }$ as a function of the maximum electron step length (Smax) for $6 \mathrm{MeV}$ and $20 \mathrm{MeV}$ monoenergetic electron beams $\left(10 \times 10 \mathrm{~cm}^{2}\right.$ field) incident on a water phantom. Voxels are $0.5 \times 0.5 \times 0.5 \mathrm{~cm}^{3}$, and the phantom has overall $\mathrm{X} \times \mathrm{Y} \times \mathrm{Z}$ dimensions of $20.5 \times 20.5 \times 30 \mathrm{~cm}^{3}$.

For the BEAMnrc simulation source, this is the time required for a full treatment head simulation, while for the phase space source, it is just the time required to transport particles through the jaws in the treatment head. See the paper by Kawrakow and Walters[8] for more information about efficiency of BEAMnrc simulation sources vs. phase space sources.

When PRESTA-I boundary crossing is used in the calculations (Figure 3.7b), the efficiencies with HOWFAR increase substantially (by a factor of $~ 2.5$ for both the phase space source and the BEAMnrc simulation source), while those with "HOWFARLESS" do not increase significantly. This indicates that use of the PRESTA-I BCA at the upper boundary of the phantom (the only boundary which could have an effect on dose efficiency in this case) does not significantly increase the efficiency of the "HOWFARLESS" calculation. As a result, when PRESTA-I boundary crossing is used, the efficiency gained by using "HOWFARLESS" is reduced to $\sim 70 \%$ for the phase space source and $~ 50 \%$ for the simulation source.

Figure 3.8 presents efficiencies for all doses $>0.5 \mathrm{D}_{\max }$ for a $40 \times 40 \mathrm{~cm}^{2} 6 \mathrm{MV}$ photon beam from the simulated Elekta SL25 accelerator as a function of n_split, with Fig. 3.8a and Fig. 3.8b depicting results with exact BCA and PRESTA-I BCA, respectively. Dose is determined in $1 \times 1 \times 1 \mathrm{~cm}^{3}$ voxels. With exact boundary crossing, the peak efficiency for a phase space source using "HOWFARLESS" is $~ 3.5$ times that using HOWFAR. In the case of a BEAMnrc simulation source, peak efficiency with "HOWFARLESS" is $\sim 3$ times greater than that with HOWFAR. Note that, unlike the $10 \times 10 \mathrm{~cm}^{2}$ field, use of "HOWFARLESS" 


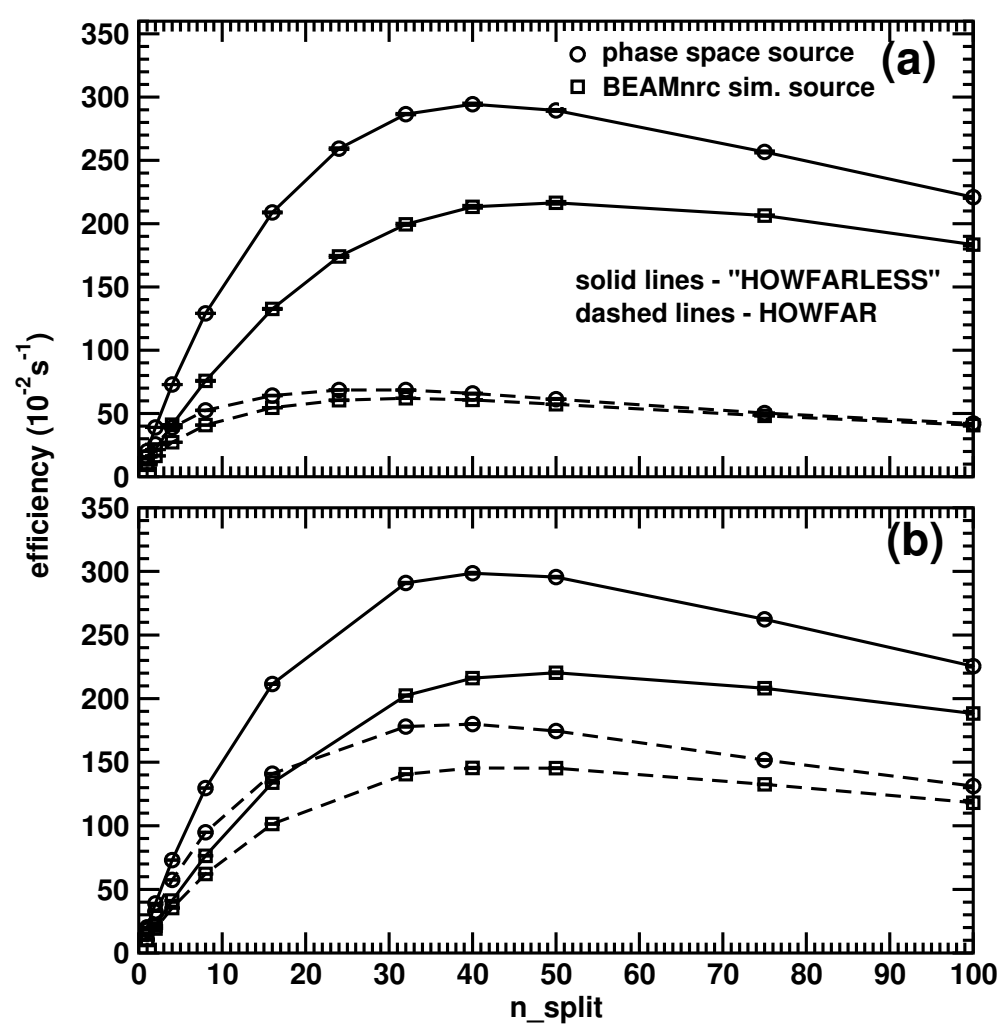

Figure 3.7: Efficiency of all doses $>0.5 \mathrm{D}_{\max }$ as a function of photon splitting number (n_split) for a $10 \times 10 \mathrm{~cm}^{2} 6 \mathrm{MV}$ photon beam from a simulated Elekta SL25 accelerator using (a) "exact" boundary crossing and (b) PRESTA-I boundary crossing. Dashed lines are doses calculated with HOWFAR and solid lines are doses calculated with the "HOWFARLESS" option. Circles indicate calculations using a phase space source, and squares indicate results using a BEAMnrc simulation source (DBS splitting no. $=1000$ ). Voxels in the DOSXYZnrc water phantom are $0.5 \times 0.5 \times 0.5 \mathrm{~cm}^{3}$ and the phantom has overall $\mathrm{X} \times \mathrm{Y} \times \mathrm{Z}$ dimensions of $20.5 \times 20.5 \times 30 \mathrm{~cm}^{3}$.

does not change the optimum value of $n_{-}$split (16 for both source types). When PRESTA-I boundary crossing is used, similar to the $10 \times 10 \mathrm{~cm}^{2}$ beam, the efficiency of the HOWFAR calculations increases substantially (by a factor of $\sim 2$ for both the phase space source and the simulation source), while that of the "HOWFARLESS" calculations does not increase significantly. As a result, the efficiency increase due to the use of "HOWFARLESS" is reduced to $70 \%$ for the phase space source and $~ 55 \%$ for the simulation source.

The peak efficiency (i.e. at the optimum photon splitting number) results for the $6 \mathrm{MV}$ Elekta photon beam and the $18 \mathrm{MV}$ Siemens KD2 photon beam $\left(10 \times 10 \mathrm{~cm}^{2}\right.$ and $40 \times 40 \mathrm{~cm}^{2}$ fields) are summarized in Table 3.1. Separate tables are shown for calculations using the exact BCA and the PRESTA-I BCA. The optimum photon splitting numbers are also given. For each beam an efficiency improvement factor 


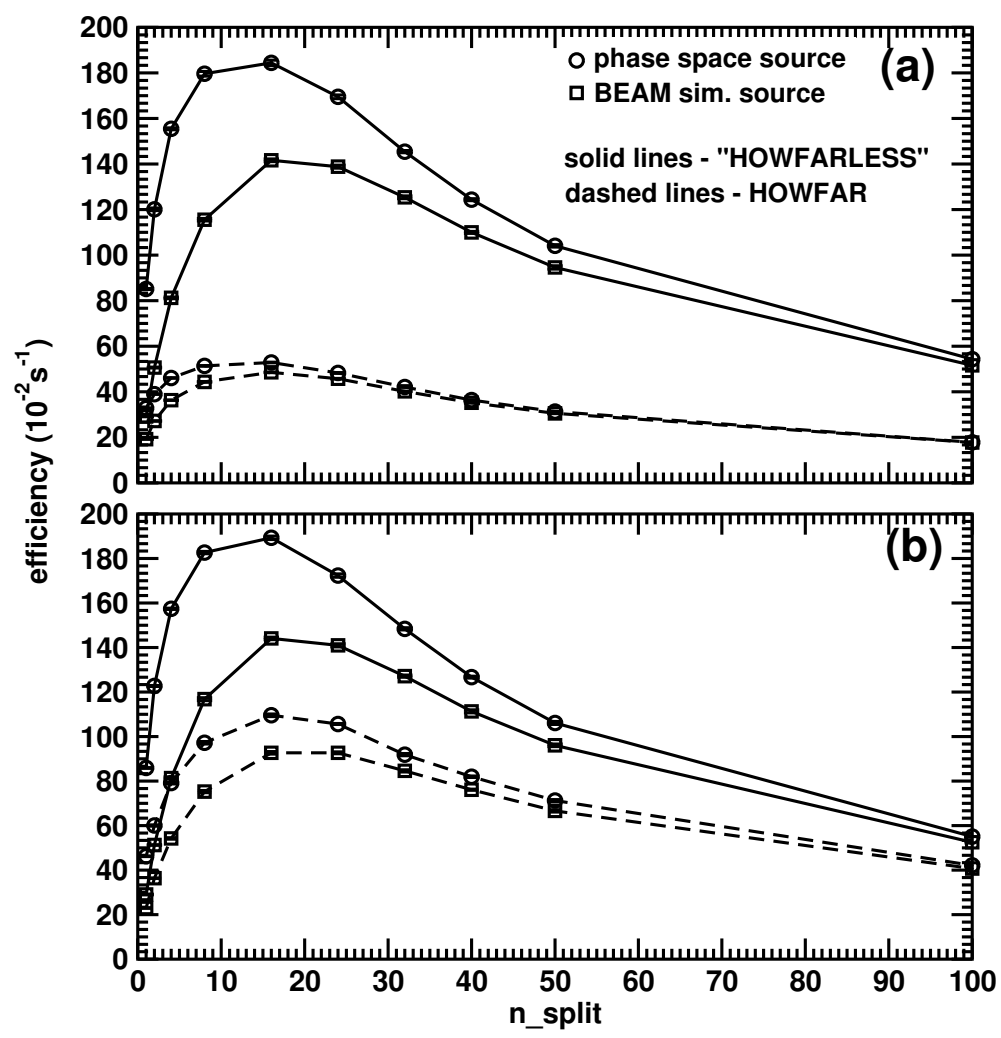

Figure 3.8: Same as figure 3.7 but with a $40 \times 40 \mathrm{~cm}^{2}$ beam and with the phantom divided into $1 \times 1 \times 1$ $\mathrm{cm}^{3}$ voxels. Overall phantom dimensions are $51 \times 51 \times 30 \mathrm{~cm}^{3}$.

("IF") due to the use of "HOWFARLESS" is found in the last row of the tables.

For the $10 \times 10 \mathrm{~cm}^{2} 18 \mathrm{MV}$ Siemens KD2 beam (case c) use of "HOWFARLESS" results in a large increase in the optimum efficiency when the exact BCA is used. As in the $6 \mathrm{MV}$ case, the relative efficiency increase for the phase space source (a factor of 5.4) is higher than that for the BEAMnrc simulation source (a factor of 4.3) due to the fact that the time to generate the source becomes more of a factor in determining efficiency as the efficiency of the phantom calculation increases, and this time is greater for the simulation source (time for a full treatment head simulation) than it is for a phase space source (time to transport particles through jaws only). When the PRESTA-I BCA is used, the increase in peak efficiency drops to $89 \%$ for the phase space source and $65 \%$ for the BEAMnrc simulation source. As in the $6 \mathrm{MV}$ beam, this is due to the large increase in efficiency in runs with HOWFAR when the BCA is switched from exact to PRESTA-I (a factor of $\sim 3$ increase in peak efficiency for both source types). Use of "HOWFARLESS" in the $40 \times 40 \mathrm{~cm}^{2} 18 \mathrm{MV}$ beam (case d-BEAMnrc simulation source only) results in an increase in peak efficiency by a factor of 3.5 with the exact BCA and by $71 \%$ with the PRESTA-I BCA. 
"exact" BCA

\begin{tabular}{|c|c|c|c|c|c|c|c|c|}
\hline \multicolumn{2}{|c|}{ Source } & \multicolumn{3}{|c|}{ Phase space } & \multicolumn{4}{|c|}{ BEAMnrc sim. } \\
\hline \multicolumn{2}{|c|}{ Case } & $\mathrm{a}$ & $\mathrm{b}$ & c & $\mathrm{a}$ & $\mathrm{b}$ & $\mathrm{c}$ & $\mathrm{d}$ \\
\hline \multirow{2}{*}{ HOWFAR } & opt. n_split & 24 & 16 & 24 & 32 & 16 & 24 & 8 \\
\hline & $\mathcal{\varepsilon}\left(10^{-2} \mathrm{~s}^{-1}\right)$ & $68.6(1)$ & $52.9(1)$ & $44.4(2)$ & $62.0(2)$ & $48.6(1)$ & $39.5(3)$ & $26.7(2)$ \\
\hline \multirow{3}{*}{ HOWFARLESS } & opt. n_split & 40 & 16 & 40 & 50 & 16 & 50 & 16 \\
\hline & $\mathcal{E}\left(10^{-2} \mathrm{~s}^{-1}\right)$ & $294.4(9)$ & $184(1)$ & $240(1)$ & $217(1)$ & $141.6(6)$ & $170.2(8)$ & $93(1)$ \\
\hline & IF & 4.3 & 3.5 & 5.4 & 3.5 & 2.9 & 4.3 & 3.5 \\
\hline
\end{tabular}

PRESTA-I BCA

\begin{tabular}{|c|c|c|c|c|c|c|c|c|}
\hline \multicolumn{2}{|c|}{ Source } & \multicolumn{3}{|c|}{ Phase space } & \multicolumn{4}{|c|}{ BEAMnrc sim. } \\
\hline \multicolumn{2}{|c|}{ Case } & a & b & $\mathrm{c}$ & $\mathrm{a}$ & b & c & $\mathrm{d}$ \\
\hline HOWFAR & $\begin{array}{l}\text { opt. n_split } \\
\varepsilon\left(10^{-2} \mathrm{~s}^{-1}\right)\end{array}$ & $\begin{array}{c}40 \\
178.0(4)\end{array}$ & $\begin{array}{c}16 \\
109.6(6)\end{array}$ & $\begin{array}{c}32 \\
129.9(6)\end{array}$ & $\begin{array}{c}40 \\
145.5(4)\end{array}$ & $\begin{array}{c}24 \\
92.7(4)\end{array}$ & $\begin{array}{c}40 \\
104.2(5)\end{array}$ & $\begin{array}{c}16 \\
57.4(2)\end{array}$ \\
\hline HOWFARLESS & $\begin{array}{c}\text { opt. n_split } \\
\varepsilon\left(10^{-2} \mathrm{~s}^{-1}\right)\end{array}$ & $\begin{array}{c}40 \\
298.6(7)\end{array}$ & $\begin{array}{c}16 \\
189.3(8)\end{array}$ & $\begin{array}{c}40 \\
245(1)\end{array}$ & $\begin{array}{c}50 \\
220.3(7)\end{array}$ & $\begin{array}{c}16 \\
144.1(6)\end{array}$ & $\begin{array}{c}50 \\
172(1)\end{array}$ & $\begin{array}{c}16 \\
98(1)\end{array}$ \\
\hline & & 1.68 & 1.73 & 1.89 & 1.51 & 1.55 & 1.65 & 1.71 \\
\hline
\end{tabular}

Table 3.1: Optimum efficiencies of all doses $>0.5 \mathrm{D}_{\max }$ calculated using HOWFAR or the "HOWFARLESS" algorithm for a simulated 6 MV Elekta SL25 photon beam with a $10 \times 10 \mathrm{~cm}^{2}$ field (a) and a $40 \times 40$ $\mathrm{cm}^{2}$ field (b) and a simulated 18 MV Siemens KD2 photon beam with a $10 \times 10 \mathrm{~cm}^{2}$ field (c) and a $40 \times 40$ $\mathrm{cm}^{2}$ field (d). The top table shows results with the "exact" BCA, and the lower table shows those with the PRESTA-I BCA. Efficiencies are shown for phase space sources (except d) and BEAMnrc simulation sources. The optimum photon splitting numbers (opt. n_split) are also shown in each case. The "IF" column shows the efficiency improvement factor due to the "HOWFARLESS" method. Doses in $10 \times 10$ $\mathrm{cm}^{2}$ beams are calculated in $0.5 \times 0.5 \times 0.5 \mathrm{~cm}^{3}$ voxels, while doses in $40 \times 40 \mathrm{~cm}^{2}$ beams are calculated in $1 \times 1 \times 1 \mathrm{~cm}^{3}$ voxels.

When the PRESTA-I BCA is used, the relative decrease in peak efficiency with increased beam field/dose voxel size is approximately the same for HOWFAR and "HOWFARLESS" calculations. For example, for the $6 \mathrm{MV}$ phase space source, use of "HOWFARLESS" increases the peak efficiency by $\sim 70 \%$ for both a $10 \times 10 \mathrm{~cm}^{2}$ field with $0.5 \times 0.5 \times 0.5 \mathrm{~cm}^{3}$ voxels (case a) and a $40 \times 40 \mathrm{~cm}^{2}$ field with $1 \times 1 \times 1$ $\mathrm{cm}^{3}$ voxels (case b). This is not the case when the exact BCA is used, however, and for the example of the $6 \mathrm{MV}$ phase space source, the peak efficiency improvement factor with "HOWFARLESS" decreases from 4.3 in the $10 \times 10 \mathrm{~cm}^{2}$ beam to 3.5 in the $40 \times 40 \mathrm{~cm}^{2}$ beam. This is due to the fact that, in the HOWFAR calculation, the increased dose voxel size in the $40 \times 40 \mathrm{~cm}^{2}$ beam decreases the fraction of the volume in which the relatively slow exact BCA is used (the BCA is used within a distance, "skin depth", of voxel boundaries, where skin depth defaults to a value of 3 electron mean free paths[6]), resulting in an increase in transport efficiency that partially offsets the decrease in efficiency due to the larger beam field.

Table 3.2 shows the dose efficiencies with HOWFAR and the "HOWFARLESS" algorithm for simple (i.e. not modeled using a BEAMnrc accelerator simulation but with a parallel beam source built-in to DOSXYZnrc) $6 \mathrm{MV}$ and $24 \mathrm{MV}$ photon beams using the energy spectra determined by Mohan et al[10] for Clinac beams of these energies (cases e and $\mathrm{f}$, respectively) and for monoenergetic electron beams with energies $6 \mathrm{MeV}$ (case g) and $20 \mathrm{MeV}$ (case h). Beam fields are $10 \times 10 \mathrm{~cm}^{2}$ in all cases. 
exact BCA

\begin{tabular}{|lcc|cc||cc|}
\hline & Case & & $\mathrm{e}$ & $\mathrm{f}$ & $\mathrm{g}$ & $\mathrm{h}$ \\
\hline \multirow{2}{*}{ HOWFAR } & & opt.n_split & 16 & 16 & $\mathrm{NA}$ & $\mathrm{NA}$ \\
& & $\varepsilon\left(10^{-2} \mathrm{~s}^{-1}\right)$ & $89.4(2)$ & $51.7(1)$ & $565(1)$ & $156.1(4)$ \\
\hline \multirow{2}{*}{ HOWFARLESS } & & opt.n_split & 16 & 4 & NA & NA \\
& & $\varepsilon\left(10^{-2} \mathrm{~s}^{-1}\right)$ & $672(2)$ & $677(3)$ & $5505(14)$ & $2682(12)$ \\
\hline & & 7.5 & 13.1 & 9.7 & 17.2 \\
\hline
\end{tabular}

PRESTA-I BCA

\begin{tabular}{|lcc|cc||cc|}
\hline & Case & & $\mathrm{e}$ & $\mathrm{f}$ & $\mathrm{g}$ & $\mathrm{h}$ \\
\hline \multirow{2}{*}{ HOWFAR } & & opt. n_split & 24 & 16 & NA & NA \\
& & $\varepsilon\left(10^{-2} \mathrm{~s}^{-1}\right)$ & $293.1(5)$ & $198.8(8)$ & $2318(9)$ & $629(2)$ \\
\hline \multirow{2}{*}{ HOWFARLESS } & & opt.n_split & 16 & 4 & NA & NA \\
& & $\varepsilon\left(10^{-2} \mathrm{~s}^{-1}\right)$ & $670(4)$ & $686(3)$ & $8055(9)$ & $3262(6)$ \\
\hline & & 2.3 & 3.5 & 3.5 & 5.2 \\
\hline
\end{tabular}

Table 3.2: Peak efficiencies of doses $>0.5 \mathrm{D}_{\max }$ calculated using HOWFAR or the "HOWFARLESS" algorithm for a simple $6 \mathrm{MV}$ photon beam $\left(10 \times 10 \mathrm{~cm}^{2}\right.$ field) with a Clinac energy spectrum (e), a simple 24 MV photon beam $\left(10 \times 10 \mathrm{~cm}^{2}\right.$ field) with a Clinac energy spectrum (f), a $6 \mathrm{MeV}$ monoenergetic electron beam $\left(10 \times 10 \mathrm{~cm}^{2}\right.$ field) $(\mathrm{g})$, and a $20 \mathrm{MeV}$ monoenergetic electron beam $\left(10 \times 10 \mathrm{~cm}^{2}\right.$ field) (h). The top table shows results with the "exact" BCA, and the bottom table shows those with the PRESTA-I BCA. In the case of photon beams, the optimum photon splitting number (opt. n_split) is also shown. Clinac energy spectra are identical to those determined by Mohan et al[10]. The "IF" row shows the efficiency improvement due to the use of "HOWFARLESS". DOSXYZnrc phantoms have $0.5 \mathrm{~cm} \times 0.5 \mathrm{~cm} \times 0.5 \mathrm{~cm}$ voxels.

Overall, the peak efficiencies both with HOWFAR and "HOWFARLESS" are significantly higher with the simple photon beams with Clinac energy spectra (cases e and $\mathrm{f}$ ) than with phase space and BEAMnrc simulation sources with similar energy and field size (cases a and $c$ in Table 3.1). This is mainly due to the fact that the time required to generate source particles in these simple photon beams does not play a significant role in the overall efficiency, as it does in the BEAMnrc simulation and phase space sources. Moreover, the efficiency improvement ("IF") due to the use of "HOWFARLESS" is greater in the simple photon beams. Compare the peak efficiency improvement factors of 7.5 (exact BCA) and 2.3 (PRESTA-I BCA) in the simple $6 \mathrm{MV}$ photon beam (case e) to the factors of 4.3 (exact BCA) and 1.68 (PRESTA-I BCA) in the $6 \mathrm{MV} 10 \times 10 \mathrm{~cm}^{2}$ phase space source (case a in Table 3.1). Efficiency gains using "HOWFARLESS" with the simple $24 \mathrm{MV}$ photon beam (case $\mathrm{f}$ ) are even higher, with peak efficiency increased by a factor of 13.1 with the exact BCA and 3.5 with the PRESTA-I BCA.

The optimum photon splitting numbers for cases e and $\mathrm{f}$ in Table 3.2 are well below those for the phase space and BEAMnrc simulation sources in Table 3.1. Again, this is due to the fact that the time required to generate source particles in the simple photon beams is insignificant compared to the time required for the phantom calculation. In the case of the BEAMnrc simulation and phase space sources, photon splitting improves efficiency mainly by decreasing the number of source particles that must be 
sampled, while with the simple photon beams, the efficiency gain observed is inherent in the photon splitting algorithm itself (and is, thus, much smaller than the gain observed with a BEAMnrc simulation or phase space source). When "HOWFARLESS" is used, optimum efficiencies tend to shift to even lower values of $n_{-}$split.

Cases $g$ and $\mathrm{h}$ in Table 3.2 show that the dose calculation efficiencies in monoenergetic electron beams are significantly higher than in photon beams, with the efficiencies in the $6 \mathrm{MeV}$ beam (case g) with HOWFAR and using "HOWFARLESS" an order of magnitude larger than their counterparts in the simple $6 \mathrm{MV}$ photon beam (case e). Moreover, the efficiency improvement factors due to the use of "HOWFARLESS" are greater than those observed in the simple photon beams. This is expected, since the "HOWFARLESS" algorithm only increases the efficiency of charged particle transport. In standard HOWFAR calculations, the efficiency with the PRESTA-I BCA relative to that with the exact BCA for monoenergetic electron beams is similar to that for the simple photon beams (cases e and $\mathrm{f}$ ). However, when "HOWFARLESS" is used, the efficiency with the PRESTA-I BCA relative to that with the exact BCA is significantly higher for the monoenergetic electron beams. For example, the efficiency of the "HOWFARLESS" calculation using the PRESTA-I BCA is $46 \%$ higher than that using the exact BCA for the $6 \mathrm{MeV}$ electron beam (case $\mathrm{g}$ ) but is the same (within uncertainty) for the $6 \mathrm{MV}$ simple photon beam (case e). This is because dose from the electron beams is concentrated at or near the extreme upper boundary of the phantom where use of the PRESTA-I BCA can actually increase the efficiency of calculations using the "HOWFARLESS" algorithm.

\subsubsection{Simulation times}

Table 3.3 summarizes the fraction of time spent delivering particles to the DOSXYZnrc simulation when using 6 and 18 MV BEAMnrc simulation sources or phase space sources. Three main conclusions can be

\begin{tabular}{|c|c|c|c|c|}
\hline \multirow{2}{*}{ Case } & \multicolumn{2}{|c|}{ HOWFARLESS } & \multicolumn{2}{|c|}{ HOWFAR } \\
\hline & exact BCA & PRESTA BCA & exact BCA & PRESTA BCA \\
\hline $6 \mathrm{MV} 10 \times 10$ phsp & 0.41 & 0.41 & 0.14 & 0.25 \\
\hline $6 \mathrm{MV} 10 \times 10 \mathrm{sim}$ & 0.49 & 0.50 & 0.18 & 0.37 \\
\hline $18 \mathrm{MV} 10 \times 10$ phsp & 0.38 & 0.39 & 0.10 & 0.24 \\
\hline $18 \mathrm{MV} 10 \times 10 \mathrm{sim}$ & 0.53 & 0.53 & 0.20 & 0.36 \\
\hline $6 \mathrm{MV} 40 \times 40$ phsp & 0.072 & 0.075 & 0.021 & 0.043 \\
\hline $6 \mathrm{MV} 40 \times 40 \mathrm{sim}$ & 0.30 & 0.30 & 0.10 & 0.15 \\
\hline $18 \mathrm{MV} 40 \times 40 \mathrm{sim}$ & 0.37 & 0.37 & 0.18 & 0.22 \\
\hline
\end{tabular}

Table 3.3: Fraction of time spent in the particle source for 6 and $18 \mathrm{MV}$ photon beams of $10 \times 10 \mathrm{~cm}^{2}$ and $40 \times 40 \mathrm{~cm}^{2}$ field sizes when using phase space sources (abbreviated with phsp) and BEAMnrc simulation sources (abbreviated with sim).

drawn from the numbers in the table:

- With the HOWFARLESS option, the fraction of time spent on obtaining particles is much larger compared to HOWFAR simulations, ranging between $\sim 30 \%$ for the large fields and $\sim 50 \%$ for the 
$10 \times 10 \mathrm{~cm}^{2}$ fields.

- Therefore, with the HOWFARLESS option, a faster treatment head simulation is about as important as a faster simulation in the phantom for further improvements of the overall efficiency. This is in contrast to the calculations with HOWFAR, where even if the treatment head simulation is a factor of 2 faster, the overall efficiency gain would only be a few percent.

- The difference in efficiency between a phase space source and a BEAMnrc simulation source is larger for the HOWFARLESS option, with a phase space source being up to $25 \%$ more efficient (this is also evident from the results in Table 3.1). Nevertheless, considering the advantage of not having to produce and store large phase space files, using a BEAMnrc simulation source remains an attractive alternative.

\subsection{Conclusions}

The new "HOWFARLESS" option in DOSXYZnrc saves significant computation time by ignoring voxel boundaries when transporting charged particles in a homogeneous phantom. The actual efficiency improvement depends on the incident beam energy and field size and the phantom voxel size. In dose calculations for realistic, BEAMnrc-simulated 6 and $18 \mathrm{MV}$ photon beams incident on homogeneous water phantoms with $0.5 \mathrm{~cm} \times 0.5 \mathrm{~cm} \times 0.5 \mathrm{~cm}$ voxels $\left(10 \times 10 \mathrm{~cm}^{2}\right.$ field $)$ or $1 \mathrm{~cm} \times 1 \mathrm{~cm} \times 1 \mathrm{~cm}$ voxels $\left(40 \times 40 \mathrm{~cm}^{2}\right.$ field), the use of "HOWFARLESS" increases the peak efficiency by a factor of 2.9-3.5 when the exact boundary crossing algorithm (BCA) is used and by $51-89 \%$ when the more efficient PRESTAI BCA is utilized (with the efficiency improvement for phase space sources being greater than that for BEAMnrc simulation sources). Thus, "HOWFARLESS" is recommended for all DOSXYZnrc calculations in homogeneous phantoms. In particular, it can save significant CPU time in beam commissioning calculations.

The smaller increase in efficiency with the PRESTA-I BCA is due to a greater relative efficiency of calculations with HOWFAR when this BCA is used. This is because, in calculations with HOWFAR, the BCA is used at all voxel boundaries, while, with the "HOWFARLESS" option, the only boundary where the BCA is used which could potentially have an effect on dose efficiency in these calculations is the upper boundary of the phantom, and, as shown in the results above, its effect on efficiency is minimal. It should be noted that, due to inaccurate modeling of lateral deflections, the PRESTA-I BCA is known to result in dose inaccuracies of up to $2 \%$ under conditions when charged particle equilibrium does not hold or when the phantom is not divided into uniform voxels throughout[20], and, thus, there are situations when use of the exact BCA is required. Moreover, even with the PRESTA-I BCA, calculations using "HOWFARLESS" are more accurate than those using HOWFAR, because the BCA is only used at the phantom outer boundaries in the former case.

The efficiency improvement with "HOWFARLESS" is greater for simpler photon beams using an energy spectrum (in this case, that of a Clinac beam[10]), and the efficiency improves by factors of 7.5 (exact BCA) and 2.3 (PRESTA-I BCA) for a $6 \mathrm{MV}$ photon beam and 13.1 (exact BCA) and 3.5 (PRESTA-I 
BCA) for a $24 \mathrm{MV}$ photon beam. The greater efficiency improvement with these simple photon sources is due to the fact that the time required to generate the source particles is negligible, making the efficiency of the DOSXYZnrc calculation the dominant factor.

The greatest gains in efficiency occur with monoenergetic electron beams, with efficiency improving by factors of 9.7 (exact BCA) and 3.5 (PRESTA-I BCA) for a $6 \mathrm{MeV}$ beam and 17.2 (exact BCA) and 5.2 (PRESTA-I BCA) for a $20 \mathrm{MeV}$ beam. This is expected, since the "HOWFARLESS" algorithm is a technique to speed up charged particle transport.

This study only investigates the effects of varying Smax, the maximum allowable charged particle step length, on accuracy when "HOWFARLESS" is used. It is found that the algorithm produces accurate results over all relevant values of Smax, thus making estepe, the maximum fractional energy loss/step, the limiting factor on maximum step length. The default value of estepe is 0.25 , which is currently the maximum value for which the PRESTA-II condensed history electron step algorithm in EGSnrc is known to be accurate[6]. Preliminary studies have shown that by increasing estepe to 0.4 , beyond which the the hinged-step approximation of the "HOWFARLESS" method results in significant inaccuracies, the efficiency of "HOWFARLESS" calculations can be increased by an additional 30\%. However, a more detailed investigation of the effects of increasing estepe is beyond the scope of this work.

\subsection{Acknowledgments}

The authors would like to acknowledge the assistance of C. Ross, P. Saull and M. McEwen from the NRCC for many useful comments on this manuscript.

\subsection{Bibliography}

[1] Indrin J. Chetty, Bruce Curran, Joanna E. Cygler, John J. DeMarco, Gary Ezzell, Bruce A. Faddegon, Iwan Kawrakow, Paul J. Keall, Helen Liu, C.-M. Charlie Ma, D. W. O. Rogers, Jan Seuntjens, Daryoush Sheikh-Bagheri, and Jeffrey V. Siebers. Report of the AAPM Task Group No. 105: Issues associated with clinical implementation of Monte Carlo-based photon and electron external beam treatment planning. Med. Phys., 34:4818-4853, 2007. http://link . aip.org/link/?MPH/34/ $4818 / 1$.

[2] F. Verhaegen and J. Seuntjens. Monte Carlo modelling of external radiotherapy photon beams. Phys. Med. Biol., 48:R107 - R164, 2003.

[3] C.-M. Ma and S. B. Jiang. Monte Carlo modelling of electron beams from medical accelerators. Phys. Med. Biol., 44:R157-R189, 1999.

[4] D. W. O. Rogers, B. Walters, and I. Kawrakow. BEAMnrc Users Manual. NRC Report PIRS 509(a)revI, 2005. 
[5] I. Kawrakow. Accurate condensed history Monte Carlo simulation of electron transport. I. EGSnrc, the new EGS4 version. Med. Phys., 27:485 - 498, 2000.

[6] I. Kawrakow and D. W. O. Rogers. The EGSnrc Code System: Monte Carlo simulation of electron and photon transport. Technical Report PIRS-701 (4th printing), National Research Council of Canada, Ottawa, Canada, 2003.

[7] B. R. B. Walters, I. Kawrakow, and D. W. O. Rogers. DOSXYZnrc Users Manual. NRC Report PIRS 794 (rev B), 2005.

[8] I. Kawrakow and B. R. B. Walters. Efficient photon beam dose calculations using DOSXYZnrc with BEAMnrc. Med. Phys., 33:3046 - 3056, 2006.

[9] Iwan Kawrakow, D. W. O Rogers, and B.R.B. Walters. Large efficiency improvements in BEAMnrc using directional bremsstrahlung splitting. Med. Phys., 31:2883 - 2898, 2004.

[10] R. Mohan, C. Chui, and L. Lidofsky. Energy and angular distributions of photons from medical linear accelerators. Med. Phys., 12:592 - 597, 1985.

[11] J. Baro, J. Sempau, J. M. Fernandez-Varea, and F. Salvat. PENELOPE: an algorithm for Monte Carlo simulation of the penetration and energy loss of electrons and positrons in matter. Nucl. Inst. Meth. $B, 100: 31-46,1995$.

[12] I. Kawrakow, M. Fippel, and K. Friedrich. 3D Electron Dose Calculation using a Voxel based Monte Carlo Algorithm (VMC). Med. Phys., 23:445 - 457, 1996.

[13] I. Kawrakow. Improved modeling of multiple scattering in the Voxel Monte Carlo model. Med. Phys., 24:505 - 517, 1997.

[14] M. Fippel. Fast Monte Carlo dose calculation for photon beams based on the VMC electron algorithm. Med. Phys., 26:1466 - 1475, 1999.

[15] I. Kawrakow and M. Fippel. Investigation of variance reduction techniques for Monte Carlo photon dose calculation using XVMC. Phys. Med. Biol., 45:2163 - 2184, 2000.

[16] I. Kawrakow. $\mathrm{VMC}^{++}$, electron and photon Monte Carlo calculations optimized for Radiation Treatment Planning. In A. Kling, F. Barao, M. Nakagawa, L. Távora, and P. Vaz, editors, Advanced Monte Carlo for Radiation Physics, Particle Transport Simulation and Applications: Proceedings of the Monte Carlo 2000 Meeting Lisbon, pages 229 - 236. Springer, Berlin, 2001.

[17] J. Sempau, S. J. Wilderman, and A. F. Bielajew. DPM, a fast, accurate Monte Carlo code optimized for photon and electron radiotherapy treatment planning dose calculations. Phys. Med. Biol., 45: $2263-2291,2000$. 
[18] I. Kawrakow and A. F. Bielajew. On the condensed history technique for electron transport. Nuclear Instruments and Methods, 142B:253 - 280, 1998.

[19] D. W. O. Rogers and R. Mohan. Questions for comparison of clinical Monte Carlo codes. In W. Schlegel and T. Bortfeld, editors, The Use of Computers in Radiotherapy, XIIIth Int'l Conf., Heidelberg, pages 120 - 122. Springer-Verlag, Heidelberg, 2000.

[20] I. Kawrakow and B. R. B. Walters. Technical note: Overprediction of dose with default PRESTA-I boundary crossing in DOSXYZnrc and BEAMnrc. Med. Phys., 34:547-650, 2007.

[21] B De Smedt, N Reynaert, W De Neve, and H Thierens. DOSSCORE: an accelerated DOSXYZnrc code with an efficient stepping algorithm and scoring grid. Phys. Med. Biol., 49:4623 - 4635, 2004.

[22] C.-M. Ma, J. S. Li, T Pawlicki, S. B. Jiang, J. Deng, M. C. Lee, T. Koumrian, M. Luxton, and S. Brain. A Monte Carlo dose calculation tool for radiotherapy treatment planning. Phys. Med. Biol., 47:1671 $-1690,2002$. 


\section{IV}

Large efficiency improvements in BEAMnrc using directional bremsstrahlung splitting 


\begin{abstract}
The introduction into the BEAMnrc code of a new variance reduction technique, called Directional Bremsstrahlung Splitting (DBS), is described. DBS uses a combination of interaction splitting for bremsstrahlung, annihilation, Compton scattering, pair production and photo-absorption, and Russian Roulette to achieve a much better efficiency of photon beam treatment head simulations compared to the splitting techniques already available in BEAMnrc (Selective Bremsstrahlung Splitting, SBS, and Uniform Bremsstrahlung Splitting, UBS). In a simulated $6 \mathrm{MV}$ photon beam $\left(10 \times 10 \mathrm{~cm}^{2}\right.$ field) photon fluence efficiency in the beam using DBS is over 8 times higher than with optimized SBS and over 20 times higher than with UBS, with a similar improvement in electron fluence efficiency in the beam. Total dose efficiency in a central-axis depth-dose curve improves by a factor of 6.4 over SBS at all depths in the phantom. The performance of DBS depends on the details of the accelerator being simulated. At higher energies, the relative improvement in efficiency due to DBS decreases somewhat, but is still a factor of 3.5 improvement over SBS for total dose efficiency using DBS in a simulated $18 \mathrm{MV}$ photon beam. Increasing the field size of the simulated $6 \mathrm{MV}$ beam to $40 \times 40 \mathrm{~cm}^{2}$ (broad beam) causes the relative efficiency improvement of DBS to decrease by a factor of $\approx 1.7$ but is still up to 7 times more efficient than with SBS.
\end{abstract}




\subsection{Introduction}

BEAMnrc [1, 2] is a widely-used Monte Carlo code for simulating radiotherapy beams (see ref [3] for a listing of over 150 publications). In the simulation of photon beams, a variance reduction technique that is often used involves "splitting" bremsstrahlung interactions, so that each bremsstrahlung event produces NBRSPL photons each having weight NBRSPL ${ }^{-1}$, where NBRSPL is the bremsstrahlung splitting number that is controlled by the user. Bremsstrahlung splitting can greatly decrease the uncertainty in all photon quantities (e.g. dose due to photons, photon fluence, photon energy spectrum) at the bottom of the accelerator for a given number of electrons incident on the photon target. The decrease in uncertainty is greater than the increase in CPU time/history required by bremsstrahlung splitting, so the overall result is an increased efficiency in photon quantities at the bottom of the accelerator. In the original version of BEAM there was an option to use uniform bremsstrahlung splitting (UBS). In 1998 an improved splitting routine, selective bremsstrahlung splitting (SBS), was added to the code and further enhanced the efficiency[4,5]. In this paper we report a further significant improvement in efficiency from an algorithm called directional bremsstrahlung splitting (DBS). We describe a specific implementation in the BEAMnrc system (which has been made available with the BEAMnrc04 release) but the techniques described are applicable in general and could be used to improve the efficiency of any Monte Carlo code for simulating photon accelerators.

\subsection{Uniform and Selective Bremsstrahlung Splitting}

In UBS, NBRSPL is set to a constant user-input value (usually between 20 and 100). On the other hand, SBS varies the value of NBRSPL to maximize splitting of photons aimed into the field and minimize unnecessary splitting of photons aimed away from the field. The value of NBRSPL for a bremsstrahlung event is based on the energy and direction of the incident electron and is proportional to the integrated probability of the bremsstrahlung photon entering a user-defined field (defined by field size, FS (width of a square field) and SSD, usually at the bottom of the accelerator). SBS also requires the user to set a lower limit on NBRSPL, called the background splitting number. Several guidelines are suggested[2] for the use of SBS, such as selection of a maximum splitting number between 200 and 1000, with a background splitting number equal to one tenth of the maximum, and selection of a field size which is $10 \mathrm{~cm}$ greater than the actual field width.

If the user is not interested in electron statistics at the bottom of the accelerator, then both UBS and SBS offer the option of playing Russian Roulette with all secondary and higher-order charged particles. This entails setting a survival probability threshold equal to NBRSPL ${ }^{-1}$ for each secondary/higher-order charged particle and comparing this threshold to a random number. If the random number is higher than the survival threshold, then the charged particle is eliminated immediately after it has been created. If the random number is less than or equal to the survival threshold, then the charged particle is kept, and its weight is increased by a factor of NBRSPL. This higher weight particle carries the transport physics for itself and all of the secondary/higher-order charged particles that were eliminated by Russian Roulette. 
Russian Roulette can increase the efficiency of UBS and SBS by a factor of 2, however, as mentioned above, the electron statistics will be compromised.

If Russian Roulette is turned on, then both UBS and SBS will split higher-order bremsstrahlung photons and photons from annihilation events (in either case, these will be photons created by higherweight charged particles that have survived Russian Roulette). In UBS, the splitting number for these events is equal to that used for primary bremsstrahlung events. In SBS, the splitting number for these higher-order events is equal to the background splitting number for primary bremsstrahlung events. If Russian Roulette is off, then UBS and SBS do not split higher-order bremsstrahlung or annihilation events to avoid spending CPU time tracking particles of vanishing weight.

Both UBS and SBS have limitations. As mentioned above, the non-directional nature of UBS means that much of the CPU time is spent tracking split photons that will not make it to the field of interest at the bottom of the accelerator. While SBS is intended to remedy this by maximizing the splitting of those photons aimed into the field of interest, statistics in the field of interest are compromised by the large range of photon weights that result from having a variable splitting number. In addition, SBS requires additional CPU time for "background splitting" of bremsstrahlung photons aimed away from the field. Background splitting was found to be necessary to prevent high-weight photons from "chance events" (e.g. photons incorrectly deemed out of the field of interest by the selective splitting function, or photons initially aimed away from the field and then scattering back into it) from compromising the statistics in the field of interest.

\subsection{Directional Bremsstrahlung Splitting}

We are introducing another bremsstrahlung splitting routine called directional bremsstrahlung splitting (DBS) into the BEAMnrc code. It is designed to overcome the limitations of SBS, by ensuring that all photons in the field of interest have the same weight and by eliminating the need for "background splitting".

Similar to SBS, DBS requires the user to define a field of interest (using the field radius and the SSD). Beyond this, however, the two algorithms diverge. The complete DBS algorithm is fairly complex. In sections 4.3.1 - 4.3.7 we present details of the algorithm that leads to large increase of the efficiency calculating the photon fluence but leads to poor statistics for contaminant electrons. The full algorithm, including improved statistics for electron contamination, is presented in section 4.3.8

\subsubsection{Bremsstrahlung Events}

In general, bremsstrahlung events initiated by a primary, or by a fat (high-weight) electron (more about fat electrons below) are split by a fixed, user-defined splitting number, NBRSPL, with the resultant photons all having weight NBRSPL ${ }^{-1}$. The algorithm then loops through all NBRSPL split photons and, for each one, determines whether or not it is aimed into the field of interest. If it is, then the photon is kept. If not, then Russian Roulette is played on the photon by comparing a random number to a survival threshold 
of NBRSPL ${ }^{-1}$. If the random number is less than this number, then the photon is kept and its weight is increased by a factor of NBRSPL. Photons aimed away from the field of interest which survive this Russian Roulette are fat and all have the same weight (normally unity).

If the user has set the EGSnrc parameters in BEAMnrc to only use the leading term of the Koch-Motz distribution for determining bremsstrahlung angles $[6,7]$ (the default in BEAMnrc), then, rather than loop through NBRSPL bremsstrahlung interactions, DBS saves further CPU time by invoking a subroutine called do_smart_brems. This subroutine determines how many of the NBRSPL split photons will be aimed into the field before the bremsstrahlung event is simulated and only generates those photons. It also samples an angle from the full distribution and, if the direction is not aimed at the field, a single fat bremsstrahlung photon travelling in this direction is generated. This fat photon is equivalent to a photon that has survived Russian Roulette in the general DBS treatment of bremsstrahlung events. Details of the equations used in the implementation of do_smart_brems are given in Appendix A. This is a proper variance reduction technique which does not bias the physics of the simulation.

\subsubsection{Annihilation Events}

If a primary, or if a fat positron (more about fat positrons below) undergoes an annihilation event, then DBS splits the event by NBRSPL, the same user-defined splitting number used to split bremsstrahlung events. Resultant photons all have weight NBRSPL ${ }^{-1}$ and, similar to bremsstrahlung splitting, the code then loops through the NBRSPL resultant photons and plays Russian Roulette with those not aimed into the field of interest. Photons which survive Russian Roulette have their weight increased (i.e. they become fat). If the positron is at rest when it annihilates, DBS uses a subroutine called uniform_photons to generate the split photons. The algorithm is similar to the algorithm of do_smart_brems described in Appendix A, except that the probability $W^{\prime}$ is simply given by $\left(\mu_{\max }-\mu_{\min }\right) / 2$ and the sampling of directions is done uniformly between $\mu_{\min }$ and $\mu_{\max }$.

\subsubsection{Compton Events}

The treatment of Compton events by DBS depends on whether the photon about to undergo the Compton event is fat (i.e. has survived Russian Roulette) or not.

If a non-fat photon is about to undergo a Compton event then: 1) if the event is about to take place in a gas (any material with $\rho \leq 0.012 \mathrm{~g} / \mathrm{cm}^{3}$ ), then DBS allows the single Compton event to proceed normally and plays Russian Roulette with the resultant Compton scattered photon if it is not aimed into the field of interest. 2) otherwise, DBS plays Russian Roulette with the photon before the event can take place. If this photon survives, then its weight is increased by a factor of NBRSPL and it becomes fat. The special treatment of the in-gas case is to prevent possible creation of fat photons immediately above the field of interest which could, in turn create a large number of non-fat photons which enter the phantom with similar characteristics.

If the photon about to undergo a Compton event is fat (either because it was fat to begin with or because it started as non-fat, but survived the Russian Roulette described above), then DBS splits the 
Compton event NBRSPL times. Generally, splitting the compton event entails calling the EGSnrc subroutine COMPT NBRSPL times, with all resulting particles having weight NBRSPL ${ }^{-1}$ times the weight of the fat photon undergoing the event. Then, DBS loops through all resultant particles, performing Russian Roulette on any Compton scattered photons not directed into the field of interest and on all secondary electrons (including from Auger and/or Coster-Kronig events). As always, particles that survive Russian Roulette have their weight increased by a factor of NBRSPL, thereby becoming fat.

If the EGSnrc bound Compton scattering option is off (i.e., the Klein-Nishina approximation, which is the BEAMnrc default, is being used), then, instead of the general Compton splitting algorithm outlined above, DBS saves CPU time by using a subroutine called do_smart_compton. In a similar way as do_smart_brems, this subroutine calculates how many of the NBRSPL compton scattered photons will have a polar angle that can direct them into the field of interest and only generates these. In addition, do_smart_compton generates a single Compton scattered photon with no angular restrictions. If this photon happens to be directed outside the field of interest, then it is kept as a fat photon with weight 1 , and carries the physics for all photons not directed into the field. On the other hand, if the photon happens to be directed into the field of interest it is unnecessary (since all of these photons have already been generated) and is discarded immediately. Finally, do_smart_compton creates a single fat electron of weight equal to the weight of the original fat photon undergoing the Compton event. More details about the equations and sampling algorithm used are given in Appendix B.

\subsubsection{Pair Production Events}

Similar to Compton events, if a non-fat photon is about to undergo a pair production event, then DBS only allows the event to take place if the photon is about to interact in a gas. Otherwise, DBS plays Russian Roulette with the photon. If the photon survives, it becomes fat, with its weight increased by a factor of NBRSPL and then undergoes the pair production event.

A fat photon is always allowed to undergo a pair production event, but the event is not split (unlike Compton). This ensures that the resultant charged particle pair will be fat (except in a gas).

\subsubsection{Photoelectric Events}

Photoelectric events are treated the same as pair production events. This ensures that all photoelectrons are fat (except in a gas).

\subsubsection{Fluorescent Photons}

After a photoelectric event, a fluorescent photon may be created. If the fluorescent photon is non-fat (from a photoelectric event undergone by a non-fat photon), then it is subject to Russian Roulette if not directed into the field of interest. If the fluorescent photon is fat (from a photoelectric event undergone by a fat photon), DBS splits it NBRSPL times isotropically using the unif orm_photons subroutine described 
in the section on annihilation events above. This ensures that any fluorescent photon reaching the field of interest is non-fat.

\subsubsection{Summary of DBS without electron splitting}

In summary, the techniques described up to this point ensure that all photons inside the circular target field will be non-fat, i.e. have a weight of 1 /NBRSPL and those outside it will have a weight 1 . If the splitting number NBRSPL is large, there will be very few fat photons that don't reach the field of interest compared to the many low-weight photons that do. Therefore only very little time will be spent transporting photons that don't contribute to the fluence in the field of interest. Similarly, all electrons will be fat except for those few created by non-fat photons interacting in the air just above where the phase-space file is created. As a result, a very small fraction of the CPU time will be spent transporting electrons.

\subsubsection{Electron splitting}

As described up to this point, the DBS technique eliminates electrons generated by split particles/interactions, either by playing Russian Roulette with them or by not generating them at all. The result is that electrons are represented by relatively few fat particles reaching the bottom of the accelerator, and therefore the contaminant electron statistics are poor. In most practical applications, one is interested in the contribution of electrons to the total dose. In order to improve the statistics of electrons, a few modifications to the DBS technique described so far are necessary.

Generally, the further away from the bottom of the accelerator the electrons are produced, the smaller their chance to reach the patient plane. In particular, primary electrons and electrons set in motion in the photon target and primary collimator virtually never arrive at the bottom of the accelerator. On the other hand, electrons set in motion in the lower portion of the flattening filter and close to the inner and lower edges of the photon jaws, have a relatively high chance of reaching the patient plane. Given these observations, the strategy for improving the statistics of contaminant electrons are clear: i) spend as little time as possible transporting electrons in the upper portion of the treatment head, i.e. use DBS as described above, and ii) increase the number of transported electrons in the lower part of the treatment head by using electron splitting and turning off Russian Roulette played before photon interactions. We will refer to these modifications as "DBS with electron splitting" in what follows.

When electron splitting is turned on, the user defines two planes perpendicular to the beam axis: the splitting plane, and the Russian Roulette plane, where the Russian Roulette plane is above the splitting plane. These two planes divide the treatment head in 3 distinct parts:

- An "upper" portion (above the Russian Roulette plane), where DBS is used as described above with the result of very few fat electrons being transported,

- A "lower" portion (below the splitting plane), where the goal is to have many low-weight electrons and no fat electrons, 
- A transitional region (between the splitting and Russian Roulette planes), where there is a mixture of fat and low-weight electrons.

The splitting plane is defined by specifying one of the BEAMnrc component modules (CMs) in the simulation and selecting an existing plane (i.e. a boundary between layers or at the top or bottom of the $\mathrm{CM}$ ) within the CM. Usually the CM specified for the splitting plane is the flattening filter, and the plane selected is close to the bottom of the filter. Fat electrons crossing the splitting plane are split NBRSPL times (and have their weight reduced by a factor of NBRSPL). There is also an option to redistribute the split electrons assuming radial symmetry about the beam axis. In most cases this improves the spatial distribution of electrons when the beam is radially symmetric above this plane.

The Russian Roulette plane is defined by specifying its $z$-position. It is usually above the splitting plane but still within the flattening filter CM. Below the Russian Roulette plane, the following modifications are applied to the DBS algorithm to maximize production of low-weight electrons:

1. Low-weight photons about to undergo pair production, Compton, or photoelectric events are allowed to interact normally. However, scattered or fluorescent photons resulting from such interactions are subjected to Russian Roulette with a probability NBRSPL ${ }^{-1}$, if they don't go towards the field of interest.

2. If a fat photon undergoes a pair production or photoelectric event, the event is split NBRSPL times to generate NBRSPL (photo-absorption) or $2 \times$ NBRSPL (pair production) low-weight charged particles.

3. If a fat photon undergoes a Compton event then the event is split NBRSPL times and Russian Roulette is not played with any resultant charged particles. The subroutine do_smart_compton is never used. The NBRSPL scattered photons originating from such split Compton events are subjected to Russian Roulette, if they don't go towards the field of interest.

Note that the non-fat charged particles generated by the electron splitting algorithm described above may, in turn, generate non-fat photons through bremsstrahlung or annihilation events. These photons are subject to Russian Roulette if they are not directed into the field of interest.

Electron splitting ensures an increase in the number of electrons in the field at a cost in the CPU time required to transport them. At the same time, all photons going towards the field of interest are still low-weight and all photons directed away from the field of interest are fat.

\subsection{Performance of Directional Bremsstrahlung Splitting}

All of the techniques applied in DBS are standard variance reduction techniques (splitting, Russian Roulette, biased sampling) and thus they do do not change the physics of the calculation and they provide an unbiased estimate of any scored quantity [8]. To ensure that we had not introduced any bugs into the coding, we made sure that the results obtained with and without the DBS algorithm were identical within good statistics for all of the efficiency tests discussed in the following and for a variety of other situations as well. Once the accuracy is established, it it critical to 'measure' the improvement in efficiency for a 
variety of situations since variance techniques are not guaranteed to improve efficiency, just to maintain accurate physics.

In order to look at the performance of DBS, we used BEAMnrc to do a full simulation of a $6 \mathrm{MV}$ photon beam from an Elekta SL25 accelerator $(10 \times 10 \mathrm{~cm}$ field at the phantom surface at SSD=100 cm) and examined fluence at the SSD and dose in a water phantom placed at the bottom of the accelerator.

Apart from the bremsstrahlung splitting parameters, simulation parameters were identical to those used by Sheikh-Bagheri and Rogers $[9,10]$. In particular, range rejection was performed on charged particles with energies $<2 \mathrm{MeV}$, with rejection occurring if the particle did not have sufficient energy to make it to the nearest region boundary. The jaws defining the field were modelled after jaws that are intended to be used with a multi-leaf collimator attachment. These jaws differed from standard jaws (which are $10 \mathrm{~cm}$ thick, comprising $5 \mathrm{~cm}$ of tungsten and $5 \mathrm{~cm}$ of lead, in both $\mathrm{X}$ and $\mathrm{Y}$ directions) in that they consisted only of tungsten with thickness $3 \mathrm{~cm}$ in the $\mathrm{Y}$ direction and $8 \mathrm{~cm}$ in the $\mathrm{X}$ direction. Thus, these jaws resulted in greater photon fluence outside the field, especially in the $\mathrm{Y}$ direction, than the standard jaws.

In all the cases presented below, performance of a bremsstrahlung splitting algorithm is specified by the efficiency, $\epsilon$, given by:

$$
\epsilon=\frac{1}{s^{2} T}
$$

where $s$ is an estimate of the uncertainty on the quantity of interest (e.g. fluence or dose) and $\mathrm{T}$ is the $\mathrm{CPU}$ time required to achieve this uncertainty.

Uncertainty was evaluated using the history-by-history method [11] which takes into account correlations between contributions to fluence or dose from particles which arise from the same initial history. This is essential when using the splitting techniques described here since each initial electron may give rise to many split photons and electrons, each contributing to the same scored quantity. Failure to take into account these correlations can result in an underestimate of the uncertainty.

\subsubsection{Fluence Scoring}

In a treatment head simulation that does not use variance reduction techniques, the uncertainty on the photon fluence within the beam for a given number of incident particles will be proportional to the inverse of the scoring zone area. The efficiency will therefore decrease with decreasing scoring zone area but the efficiency divided by the scoring zone area will be a constant. This is not necessarily the case in simulations where variance reduction techniques such as bremsstrahlung splitting are used. The smaller the scoring zone is, the smaller the probability will be that two or more particles that result from the same incident electron history will reach the zone. Ultimately, if the scoring zones are made infinitely small, correlations between particles from the same history will become negligible (the scoring zone is either reached by a single particle or not reached at all, this is the same as when no splitting was applied). Correlations between particles from the same incident electron history will modify the uncertainty compared to a situation where the same number of particles reaches the scoring plane but all 
particles are statistically independent. Depending on the nature of the correlation, the uncertainty may increase or decrease.

Given the above observations, one should score quantities such as dose or fluence in scoring zones of size that is relevant for treatment planning applications (i.e. squares or cubes of $5 \mathrm{~mm}$ or less) in order to be able to reliably assess the performance of a particular variance reduction technique. Unfortunately, BEAMnrc simulations without any variance reduction techniques used (which is our baseline) take a prohibitively long time to obtain reasonable statistical uncertainty in such small voxels. We have therefore selected slightly larger scoring zone sizes: $41 \times 411 \times 1 \mathrm{~cm}^{2}$ scoring zones for the photon fluence and $21 \times 11$ $2 \times 4 \mathrm{~cm}^{2}$ scoring zones for the electron fluence in a plane perpendicular to the beam axis at SSD=100 $\mathrm{cm}$.

To assess the influence of the selected scoring zone size on the efficiency of the various splitting techniques relative to using no splitting, we varied the size of the central-axis fluence scoring zone for simulations performed with typical optimized parameters (see section 4.4.2 for discussion of parameter selection). Figure 4.1 presents these results of relative photon fluence efficiencies. Each curve has been divided by the numbers shown in the figure $(50.6,11.5$ and 7.37) to emphasize the shape of each curve (and to normalize the values for the $6 \times 6 \mathrm{~cm}^{2}$ scoring zone to 1.00). One must note that we are presenting the relative efficiencies, and that for the no-splitting case, the absolute efficiency decreases almost exactly proportionally to the area of the scoring zone. However, it is clear that this is not the case for the bremsstrahlung splitting routines and that in particular the efficiency of the DBS algorithm decreases by almost an order of magnitude less going from the largest to smallest scoring zone sizes and thus the relative efficiency increases. The crucial point that figure 4.1 demonstrates is that the decrease of correlations with decreasing scoring zone size implies that the relative efficiency for DBS goes up substantially more than for UBS or SBS as the scoring region decreases in size. It is therefore clear that DBS will perform better relative to SBS or UBS when using scoring zone sizes typical for dose calculations in modern radiotherapy treatment planning ( $5 \mathrm{~mm}$ or less) and therefore the DBS efficiency results presented here represent a lower bound on the improvement in simulation efficiency in practical calculations. A rigorous explanation of the behaviour observed in Fig. 4.1 has been found and will be presented elsewhere.

Throughout this paper, results are presented relative to the efficiency for the baseline calculation with no splitting being used. This baseline efficiency varies quite dramatically, generally with a shape corresponding to the dose or fluence profile of interest. For example, the efficiency outside the beam is much lower because of the reduced number of photons involved.

\subsubsection{Selecting Splitting Parameters}

Setting of splitting parameters for each of the bremsstrahlung splitting routines was heavily weighted towards optimizing photon fluence efficiency, since photons were the greatest contributors to fluence or dose in our modelled accelerator. However, we also examined electron fluence efficiency, and, in the case of DBS with electron splitting, took it into account when selecting the best position of the splitting and Russian Roulette planes. 


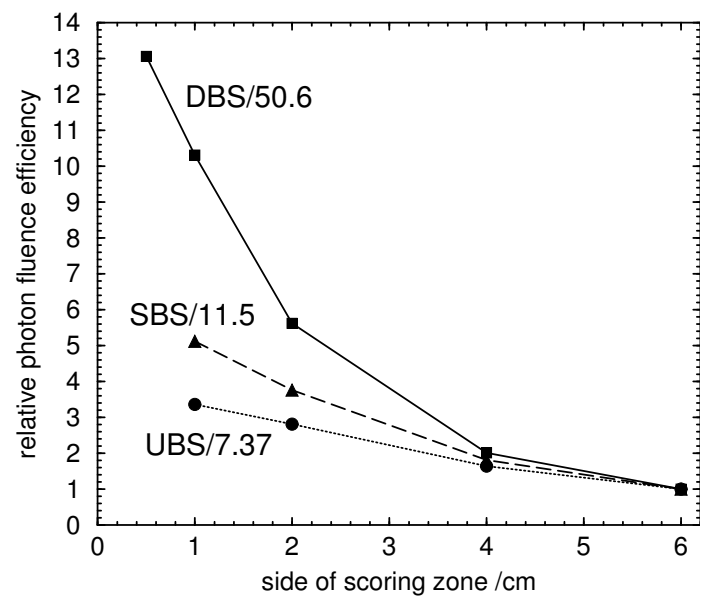

Figure 4.1: Photon fluence efficiency for the three bremsstrahlung splitting techniques (DBS, directional, SBS, selective and UBS, uniform bremsstrahlung splitting) relative to that for no splitting as a function of the side of the square scoring region on the central axis. An Elekta SL25 $6 \mathrm{MV} 10 \times 10 \mathrm{~cm}^{2}$ photon beam is simulated. To emphasize the shapes of the curves, the results are normalized to the relative efficiency for the $6 \times 6 \mathrm{~cm}^{2}$ scoring zone size in each case by dividing the results by the values shown in the figure. The relative efficiency is a strong function of the size of the scoring region, the relative improvement increasing as the scoring area decreases (although the absolute efficiency decreases with the area of the scoring region for the no-splitting case).

Rather than examine the fluence efficiency in each scoring zone while setting parameters, we examined the total efficiency in all scoring zones completely contained within the $10 \times 10 \mathrm{~cm}^{2}$ field of the beam. In the case of photon fluence this would comprise 81 of the $1 \times 1 \mathrm{~cm}^{2}$ zones, and in the case of electron fluence 5 of the $2 \times 4 \mathrm{~cm}^{2}$ zones. The square of the uncertainty used in calculating total efficiency was simply equal to the sum of the squares of the absolute uncertainty in each of these scoring zones.

\subsubsection{Selecting the splitting number (NBRSPL)}

Figure 4.2 shows the total photon fluence efficiency in the beam field vs bremsstrahlung splitting number (NBRSPL) for UBS, SBS and DBS. Efficiencies have been normalized to the total photon fluence efficiency with no splitting. In the case of UBS and SBS, results are shown with Russian Roulette on (empty circles) and off (filled circles). SBS was run with a splitting field size, FS, parameter of $30 \mathrm{~cm}$, which is the value used by Sheikh-Bagheri and Rogers $[9,10]$ in their simulations of Elekta photon beams. Results for DBS are shown with no electron splitting (empty circles) and with electron splitting on with the splitting plane at $Z=15.46 \mathrm{~cm}$ and the Russian Roulette plane at $Z=15.2 \mathrm{~cm}$. Results with electron splitting on and the splitting plane at $Z=15.66 \mathrm{~cm}$ (the very bottom of the flattening filter) and the Russian Roulette plane at $\mathrm{Z}=15.5 \mathrm{~cm}$ are indistinguishable from the results shown. We therefore assume that the trend in photon fluence efficiency vs NBRSPL is independent of the placement of the splitting and Russian Roulette planes 
in DBS.

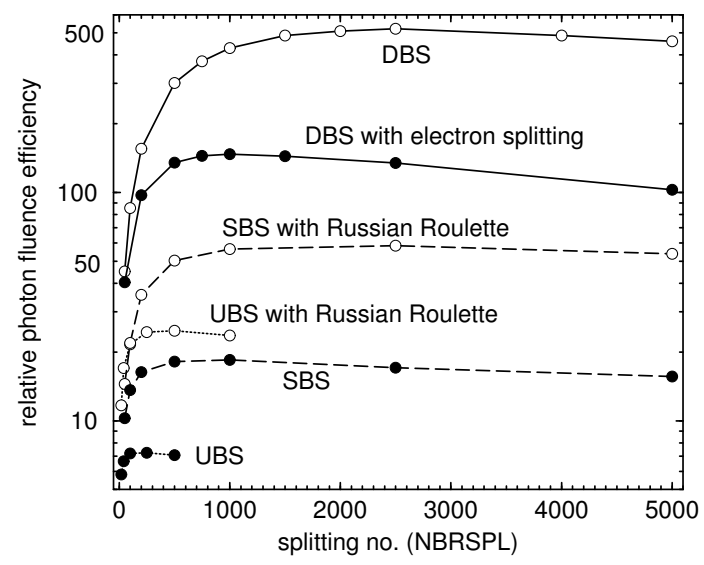

Figure 4.2: Relative efficiency for calculating photon fluence within the $10 \times 10 \mathrm{~cm}^{2}$ field of a simulated Elekta SL25 $6 \mathrm{MV}$ photon beam as a function of bremsstrahlung splitting number (NBRSPL). Efficiencies shown are relative to total photon fluence efficiency with no splitting. For UBS and SBS, efficiencies are shown with Russian Roulette on (open circles) and off (solid circles). The field size parameter, FS, used with SBS was $30 \mathrm{~cm}[9,10]$. For DBS, results are shown with electron splitting off (open circles) and electron splitting on with the splitting plane at $Z=15.46 \mathrm{~cm}$ and the Russian Roulette plane at $Z=15.2 \mathrm{~cm}$ (closed circles). For UBS the minimum splitting number is 20 and for SBS and DBS it is 50. Note the $\mathrm{y}$-axis is logarithmic.

From Figure 4.2 it is clear that DBS, with or without electron splitting, is a significant improvement over UBS and SBS. The maximum improvement in photon fluence efficiency using DBS (with NBRSPL $=2500$, no electron splitting) is a factor of 500, over 8 times the maximum efficiency using SBS (with NBRSPL=2500 and Russian Roulette on) and 20 times that achieved using UBS (with NBRSPL=750 and Russian Roulette on). For all splitting algorithms, the production of electrons (either by turning Russian Roulette off or by turning electron splitting on) results in a decrease in photon fluence efficiency by a factor of 3 and a shift of the splitting number (NBRSPL) at which the photon efficiency peaks toward lower values.

Since users are generally interested in electrons at the bottom of the accelerator, Figure 4.2 suggests using a splitting number of 100 for peak photon efficiency in UBS. The original BEAM paper [1] suggests using NBRSPL values in the range 10 - 20, but at that time, when Russian Roulette was used, secondary fat electrons were not split as they are now. The previously suggested range would result in a photon efficiency well below the maximum and implies that the most recent implementation of UBS in BEAMnrc, which uses the built-in bremsstrahlung splitting function in EGSnrc [7], is more efficient than UBS in older versions of BEAM (up to and including BEAM00).

In the case of SBS, Figure 4.2 indicates that maximum photon efficiency is achieved with NBRSPL=1000. It is important to note that, in SBS, NBRSPL represents the maximum possible splitting number, and that 
the actual bremsstrahlung splitting number is a calculated value falling somewhere between the userinput minimum splitting number, NMIN, (given its suggested value of NBRSPL/10 in all of these simulations), and NBRSPL. The BEAMnrc Manual [2] suggests a value of NBRSPL in the range $200-1000$, which is certainly reasonable given that there is little variation in photon fluence efficiency over this range.

Figure 4.2 indicates the splitting number for maximum photon efficiency in DBS (with electron splitting) is 1000 . Additional results with the electron splitting plane at $Z=15.66 \mathrm{~cm}$ and the Russian Roulette plane at $\mathrm{Z}=15.5 \mathrm{~cm}$ indicate that this optimum value of NBRSPL does not change with the positions of the these planes. Positioning of the splitting and Russian Roulette planes will be discussed in more detail below.

The electron fluence efficiencies as a function of bremsstrahlung splitting number are shown in Figure 4.3 relative to the total electron fluence efficiency with no splitting. To generate electrons with UBS and SBS, Russian Roulette was turned off. As in the plot of photon fluence efficiency vs NBRSPL, the splitting field size, FS, was set to $30 \mathrm{~cm}$. In the case of DBS, electron splitting was turned on and two sets of splitting plane and Russian Roulette plane positions were used to demonstrate that the behaviour of total electron fluence efficiency as a function of NBRSPL follows the same trend independent of the setting of these two parameters. Note that single points are shown for DBS (NBRSPL=1000) with a splitting plane $(Z=15.66 \mathrm{~cm})$ but no Russian Roulette plane and with a Russian Roulette plane $(Z=14.9 \mathrm{~cm})$ but no splitting plane.

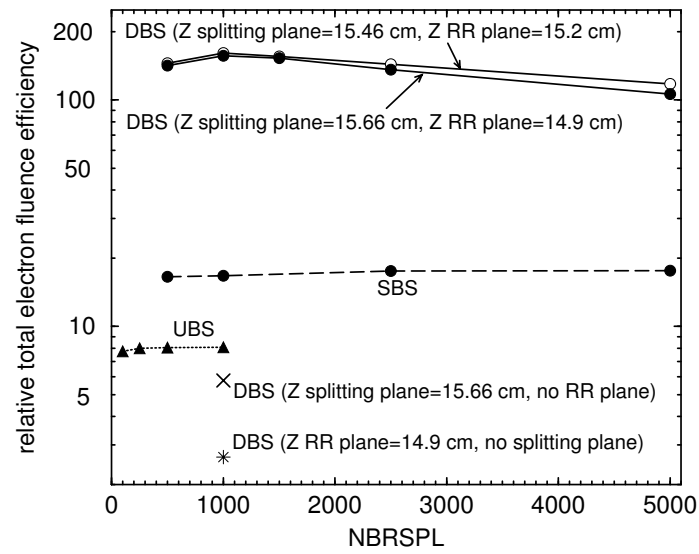

Figure 4.3: Total electron fluence efficiency in the $10 \times 10 \mathrm{~cm}^{2}$ field of a simulated Elekta SL25 6 MV photon beam as a function of bremsstrahlung splitting number (NBRSPL). Efficiencies shown are relative to total electron fluence efficiency with no splitting. In the cases of UBS and SBS, Russian Roulette was turned off. SBS was run with a splitting field size parameter, FS of $30 \mathrm{~cm}$. DBS was run with electron splitting and the splitting and Russian Roulette (RR) plane locations indicated in the figure. Note the single points indicating efficiency with DBS at NBRSPL $=1000$ with no Russian Roulette plane ("X") and with a Russian Roulette plane but no charged particle splitting (“‘”).

Figure 4.3 shows that using DBS can result in 8 times greater electron fluence efficiency than SBS and 
20 times greater efficiency than UBS. In the case of UBS and SBS, electron fluence efficiency shows little variation with NBRSPL over the range of splitting numbers studied. This means that the splitting number for maximum photon fluence efficiency (100 in the case of UBS and 1000 in the case of SBS) is adequate for electron efficiency as well. Directional bremsstrahlung splitting, on the other hand, has a definite maximum in electron fluence efficiency occurring at NBRSPL $=1000$. This is also the splitting number for maximum photon fluence efficiency when using DBS. The two DBS curves, each with different $Z$ positions of the splitting and Russian Roulette planes, indicate that the placement of these planes has little effect on the behaviour of electron fluence efficiency as a function of NBRSPL. The point with no Russian Roulette plane indicates that the Russian Roulette plane is essential for good electron statistics with DBS, with the addition of the Russian Roulette plane increasing electron fluence efficiency by a factor of almost 30 at NBRSPL $=1000$. Conversely, the point with no splitting plane illustrates that it is necessary to split fat charged particles. If they reach the field then they can decrease the efficiency by a factor of $\approx 80$.

\subsubsection{Selecting splitting field size (FS and splitting radius)}

Another consideration when using SBS and DBS is selection of the splitting field size at the bottom of the accelerator. In SBS, the user is asked to input a field size parameter, FS, which the BEAMnrc Users Manual [2] suggests setting equal to the longest side of the treatment field plus $10 \mathrm{~cm}$. In DBS, the user inputs a field radius which must include, as a minimum, the entire treatment field.

Figure 4.4 shows the relative efficiency when scoring total photon and electron fluence within the $10 \times 10 \mathrm{~cm}^{2}$ field of the SL25 $6 \mathrm{MV}$ photon beam as a function of FS for SBS and splitting field radius for DBS. Since we were interested in generating electrons, Russian Roulette was turned off in SBS, and electron splitting was turned on in DBS ( $Z$ of splitting plane $=15.66 \mathrm{~cm}, \mathrm{Z}$ of Russian Roulette plane $=15.5$ $\mathrm{cm}$ ). The bremsstrahlung splitting number (NBRSPL) was set to 1000 for both SBS and DBS, since this was shown to give maximum photon fluence efficiency when electrons were generated (see section 4.4.2.1).

For SBS, Figure 4.4 shows that photon fluence efficiency peaks at $\mathrm{FS}=40 \mathrm{~cm}$, although there is little variation in efficiency beyond $\mathrm{FS}=30 \mathrm{~cm}$. Electron fluence efficiency increases constantly over the range of FS values studied, but also shows little variation over a wide range of FS. Based on maximizing photon fluence efficiency, the setting of FS $=30 \mathrm{~cm}$ used by Sheikh-Bagheri and Rogers $[9,10]$ (and used elsewhere in this study) is certainly adequate, but the value of FS suggested by the BEAMnrc Manual (treatment field size $+10 \mathrm{~cm}=20 \mathrm{~cm}$ ) is slightly low.

In the case of DBS, Figure 4.4 shows that photon fluence efficiency constantly decreases with increasing splitting field radius. This behaviour is expected since increasing the splitting field size increases the number of events that must be split. Electron fluence efficiency, on the other hand, shows a peak at splitting radius $=10 \mathrm{~cm}$. From the point of view of maximizing photon fluence efficiency, it would seem that the optimum splitting radius would be the smallest that completely encompasses the $10 \mathrm{x} 10 \mathrm{~cm}$ treatment field $(\mathrm{eg} \approx 7.1 \mathrm{~cm}$ ). However in this particular accelerator, significant contributions to dose are made by photons out to a radius of $10 \mathrm{~cm}$. Thus, we use a splitting radius of $10 \mathrm{~cm}$ for the rest of the study. This 


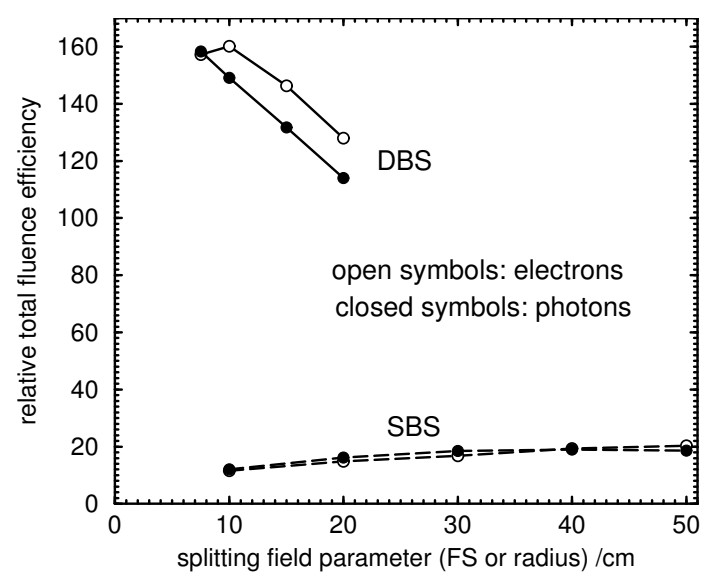

Figure 4.4: Total photon (closed circles) and electron (open circles) fluence efficiency inside the $10 \times 10$ $\mathrm{cm}^{2}$ field of the simulated Elekta SL25 $6 \mathrm{MV}$ photon beam versus the size of the bremsstrahlung splitting field (FS (width of a square field) for SBS and radius for DBS). Efficiencies shown are relative to their counterparts with no splitting. SBS was run with Russian Roulette off and DBS was run with electron splitting on, with the splitting plane at $Z=15.66 \mathrm{~cm}$ (the back of the flattening filter) and the Russian Roulette plane at $Z=15.5 \mathrm{~cm}$. The splitting number (NBRSPL) for both SBS and DBS was 1000, which was shown to give maximum photon fluence efficiency when electrons are generated.

results in only a $\approx 6 \%$ drop in photon fluence efficiency compared to a splitting radius of $7.5 \mathrm{~cm}$ and also maximizes electron fluence efficiency.

\subsubsection{Selecting Position of Splitting and Russian Roulette Planes (DBS)}

Further degrees of freedom are available in DBS for optimizing photon and electron fluence efficiency, $v i z$, the setting of the positions of the electron splitting and Russian Roulette planes. The function of these planes is described in detail in Section 4.3.8. The splitting plane should be set close to the bottom of the flattening filter to maximize the number of electrons reaching the bottom of the accelerator while minimizing the time spent transporting them in such structures as the primary collimator and the flattening filter itself. The Russian Roulette plane, below which Russian Roulette is not played on electrons resulting from interactions and low-weight photons are allowed to interact, should be placed above the splitting plane. Due to the way electron splitting is coded, the splitting plane is restricted to being coincident with a geometrical plane in the BEAMnrc component module in which it is located. Thus, in the flattening filter, this plane must coincide with a layer boundary in the modelled flattening filter or with the planes defining the top or bottom of the flattening filter. There is no such restriction on the position of the Russian Roulette plane.

Figure 4.5 shows the relative total photon and electron fluence efficiency inside the $10 \times 10 \mathrm{~cm}^{2}$ field of the simulated SL25 $6 \mathrm{MV}$ photon beam as a function of the position of the Russian Roulette plane. 
Curves are shown for splitting plane $\mathrm{Z}$ positions of $14.9 \mathrm{~cm}$ and $15.46 \mathrm{~cm}$, both corresponding to layer boundaries in the modelled flattening filter, and for splitting plane $\mathrm{Z}=15.66 \mathrm{~cm}$, corresponding to the bottom of the flattening filter. The Russian Roulette plane was always placed above the splitting plane. The splitting number (NBRSPL) was set to 1000 (shown above to maximize photon and electron fluence efficiency in DBS) and the splitting field radius was $10 \mathrm{~cm}$.

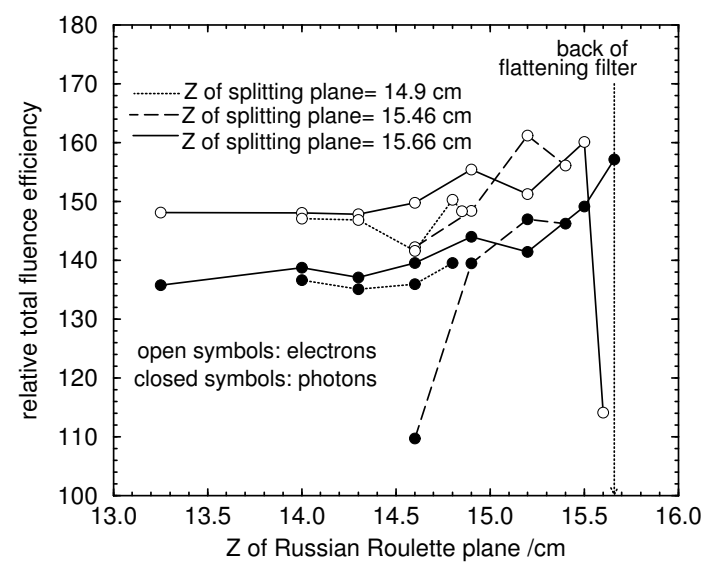

Figure 4.5: Total photon (closed circles) and electron (open circles) fluence efficiency inside the $10 \times 10$ $\mathrm{cm}^{2}$ field of the simulated Elekta SL25 $6 \mathrm{MV}$ photon beam versus the Z position of the Russian Roulette plane in DBS. Photon and electron efficiencies shown are relative to photon and electron efficiencies with no splitting. Curves are shown for 3 different $Z$ positions of the electron splitting plane: $14.9 \mathrm{~cm}$, $15.46 \mathrm{~cm}$ and $15.66 \mathrm{~cm} . \mathrm{Z}=14.9 \mathrm{~cm}$ and $\mathrm{Z}=15.46 \mathrm{~cm}$ correspond to layer boundaries in the modelled flattening filter, while $Z=15.66 \mathrm{~cm}$ corresponds to the plane defining the bottom of the flattening filter. For these simulations, the splitting number was 1000 (shown to give maximum photon and electron fluence efficiency in DBS) and the splitting field radius was $10 \mathrm{~cm}$.

The curves show that photon fluence efficiency increases as the splitting plane is brought closer to the Russian Roulette plane and also as both planes are brought closer to the bottom of the flattening filter $(15.66 \mathrm{~cm})$. Ignoring the outlier at splitting plane $\mathrm{Z}=15.46 \mathrm{~cm}$ and Russian Roulette plane $\mathrm{Z}=14.6 \mathrm{~cm}$, the overall variation in photon fluence efficiency is $\approx 16 \%$ over the range of splitting and Russian Roulette plane positions studied. Note that, again not considering the outlying point, the photon efficiency for a given Russian Roulette plane position does not change appreciably with splitting plane position.

Electron fluence efficiency shows a similar trend to photon fluence efficiency with the exception that there is a drop off in efficiency once the splitting plane is very close to (within $0.06 \mathrm{~cm}$ of) the Russian Roulette plane. This is especially noticeable in the case with the Russian Roulette plane at $Z=15.66 \mathrm{~cm}$ and the splitting plane at $Z=15.6 \mathrm{~cm}$. Ignoring this extreme point, though, the overall change in electron fluence efficiency is $\approx 12 \%$ over the range of plane positions studied.

Based on the above results, we selected a splitting plane position of $Z=15.66 \mathrm{~cm}$ (i.e. at the base of the flattening filter) with the Russian Roulette plane at $Z=15.5 \mathrm{~cm}$. This results in near-maximum values 
of the photon and electron fluence efficiency while not sacrificing one efficiency for the other (as done with splitting plane $Z=15.66 \mathrm{~cm}$ and Russian Roulette plane $Z=15.6 \mathrm{~cm}$, or splitting plane $Z=15.46 \mathrm{~cm}$ with Russian Roulette plane $Z=15.2 \mathrm{~cm}$ ). However, exact placement of these planes is not critical as long as they are near the back of the flattening filter, since the variation in both photon and electron fluence efficiencies with the positions of the splitting and Russian Roulette planes tends to be relatively small.

It is important to note that the curves shown in Figure 4.5 are not general, and the behaviour of photon and electron fluence efficiencies with splitting and Russian Roulette plane positions will most likely depend upon the flattening filter model and the beam energy.

\subsubsection{Fluence Efficiency Profiles}

Figure 4.6 shows the relative fluence efficiency for photons (a) and electrons (b) as a function of $\mathrm{X}$ at $\mathrm{Y}=0$ at the SSD $(100 \mathrm{~cm})$ of the simulated SL25 $6 \mathrm{MV}$ photon beam. The splitting parameters used were those determined based on results in Section 4.4.2 above.

Photon fluence efficiency profiles are constant within the $10 \times 10 \mathrm{~cm}^{2}$ beam field (i.e. from $\mathrm{X}=-5$ to $5 \mathrm{~cm}$ ) with the relative efficiency approximately equal to the relative efficiency totalled over the entire beam field for the same splitting routine/parameters (see section 4.4 .2 above). Beyond the beam field the UBS efficiency profile remains constant at a factor of $\approx 7$ times the efficiency with no splitting. This illustrates the main limitation of UBS, in which bremsstrahlung splitting is equal in all directions. In the case of SBS, selective bremsstrahlung splitting causes the photon fluence efficiency to drop by a factor of $\approx 2$ beyond the edges of the field. Even so, the efficiency outside the field remains quite high due to the fact that, with FS $=30 \mathrm{~cm}$, the splitting field goes well beyond the edges of the field, and to the fact that even photons aimed beyond the edges of the splitting field are split by the background splitting number (NMIN). The DBS efficiency profile, on the other hand, falls off to very low values ( $\approx 0.3$ times the efficiency with no splitting) beyond the edge of the splitting field $(\mathrm{r}=10 \mathrm{~cm})$ since no splitting is done there. The large variations in the DBS efficiency profile between the edge of the field and the edge of the splitting field are due to the fact that the fluence with no splitting, used to normalize these profiles, has large uncertainties in this region.

In contrast to the photon fluence efficiency, electron fluence efficiency profiles (Figure 4.6(b)) are almost constant over the entire range of $\mathrm{X}$ values for all splitting routines, with the relative electron efficiency approximately equal to the relative electron efficiency totalled over the beam field in Section 4.4.2 for the same splitting routine/parameters.

\subsubsection{Dose Efficiency}

Central-axis relative efficiencies for calculating does as a function of depth in phantom for the simulated $6 \mathrm{MV}$ beam using the splitting parameters optimized for photon and electron fluence efficiency are shown in Figure 4.7. Figure 4.7(a) shows total dose efficiencies and Figure 4.7(b) shows the efficiencies of the dose contributions of photons and electrons separately. Normalized depth-dose profiles are also shown in Figure 4.7. 

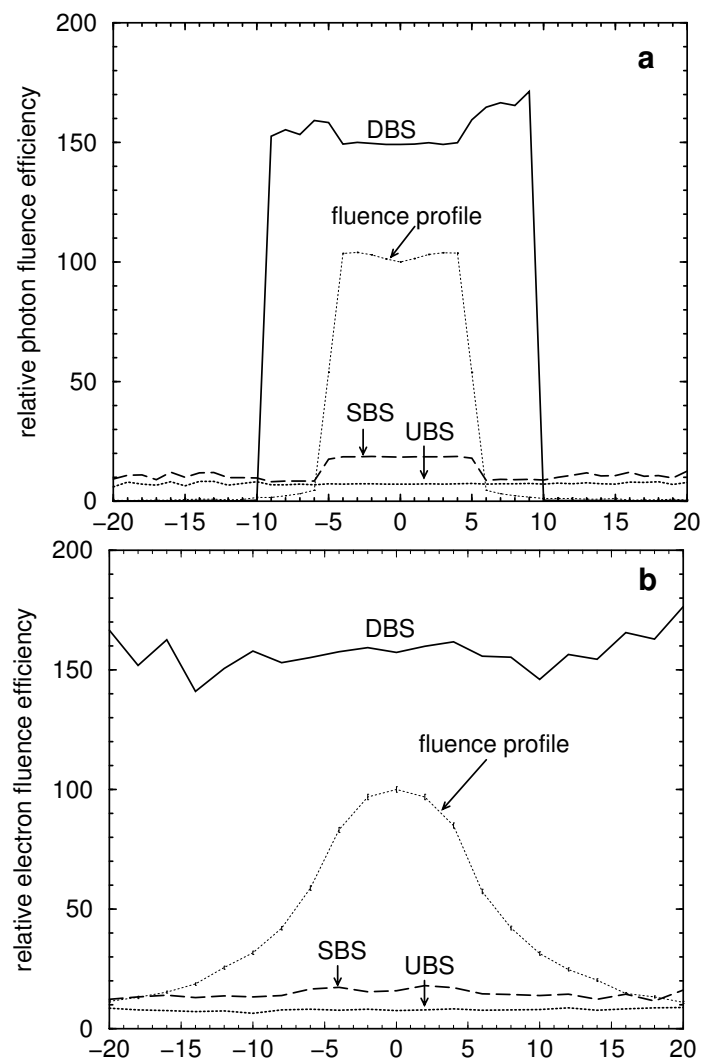

Figure 4.6: Fluence efficiency for photons (a) and elecfmons (b) vs X at $\mathrm{Y}=0$ at the SSD $(100 \mathrm{~cm})$ of the simulated Elekta SL25 $6 \mathrm{MV}$ photon beam $\left(10 \times 10 \mathrm{~cm}^{2}\right.$ field). Photon fluence was scored in $1 \times 1 \mathrm{~cm}^{2}$ zones, and electron fluence was scored in $2 \times 4 \mathrm{~cm}^{2}$ zones. Efficiencies are relative to their counterparts with no splitting. UBS was run with Russian Roulette off and NBRSPL $=250$. SBS was run with Russian Roulette off, NBRSPL $=1000$ and FS $=30 \mathrm{~cm}$. DBS was run with NBRSPL $=1000$, splitting field radius $=10 \mathrm{~cm}$, and electron splitting on with splitting plane $Z=15.66 \mathrm{~cm}$ and Russian Roulette plane $Z=15.5 \mathrm{~cm}$. The photon and electron fluence profiles are also shown. 

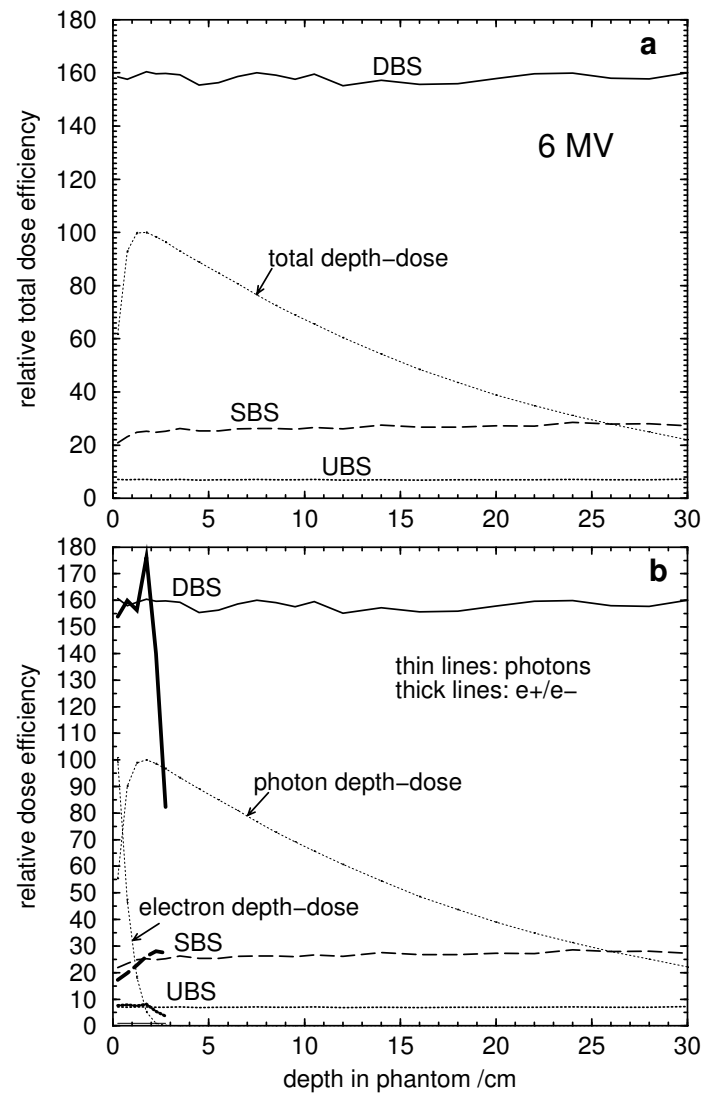

Figure 4.7: Efficiency of central-axis dose as a function of depth in phantom for the simulated $6 \mathrm{MV}$ SL25 photon beam $\left(10 \times 10 \mathrm{~cm}^{2}\right.$ field). Dose scoring volumes had radii $1 \mathrm{~cm}$ and thicknesses in the range $0.5-2 \mathrm{~cm}$. Efficiencies have been normalized by the efficiency with no splitting. (a) shows total dose efficiency and (b) shows efficiency of dose contributions from photons and charged particles (thick lines) separately. Normalized depth-dose profiles are also shown for all cases. Splitting parameters are the same as in Figure 4.6. 
An inherent feature of the DBS technique is the occurrence of fat photons outside the splitting field radius. We have found that these fat photons can introduce a very large uncertainty in the dose in a phantom, despite the fact that these photons contribute a very small fraction of the dose in the beam. We have therefore added an option to our codes for scoring dose in a phantom (ie DOSXYZnrc and the CHAMBER CM in BEAMnrc) which allows us to ignore the dose from these fat photons. In the case of DBS in figure 4.7, contributions from fat photons that enter the phantom from outside the splitting field radius $(10 \mathrm{~cm})$ have been excluded. In separate calculations we have shown that these photons only contribute about $0.1 \%$ of the dose maximum to the dose in the phantom. At the same time they lead to large fluctuations in the efficiency of the dose calculation since a few photons carry so much weight. It is clear that the size of the contribution from excluded fat photons must be determined in each situation, to ensure they are not of importance. It may be necessary to increase the splitting field radius to ensure that their contribution to dose can be safely ignored and, thus, the efficiency of the dose calculation increased.

In the case of total dose efficiency, Figure 4.7(a) shows that, for all splitting routines, the improvement in efficiency is essentially constant over all depths in the phantom. DBS resulted in the largest efficiency gain, with an improvement by a factor of 6 over SBS and an improvement by a factor of 23 over UBS. The photon dose efficiencies shown in Figure 4.7(b) are almost indistinguishable from the total dose efficiencies, since the total dose is almost entirely comprised of photons. There is some contribution from electrons at the surface, however, and over the range of depths in which electron dose is significantly greater than zero $(0-2.5 \mathrm{~cm})$, the electron dose efficiency gain for a given splitting routine is similar to the photon dose efficiency gain. There are larger variations in electron dose efficiency (especially visible in the case of DBS) since the uncertainties in electron dose are relatively high.

To confirm that optimizing parameters for fluence efficiency resulted in the maximum dose efficiency we also examined dose efficiency in the phantom while varying several of the splitting parameters in DBS. Of these parameters, only the splitting number, NBRSPL, had an effect on dose efficiency.

Figure 4.8 shows relative total dose efficiency in the phantom when using DBS with several different values of NBRSPL in the simulated 6 MV SL25 photon beam. Figure 4.8(a) shows the central axis dose efficiency, and Figure 4.8(b) shows dose efficiency as a function of radius in the phantom at $\mathrm{d}_{\max }(1.75 \mathrm{~cm}$ depth - closed circles) and near the surface of the phantom ( $0.25 \mathrm{~cm}$ depth - open circles). Normalized dose profiles at the two depths are also shown in Figure 4.8(b) for reference. This figure emphasizes that the efficiency improvement depends strongly on exactly what quantity is of interest. Although our setting of NBRSPL $=1000$ does not maximize dose efficiency on the central axis, it does maximize efficiency at all other radii near the surface (which is consistent with the fact that NBRSPL=1000 resulted in maximum total fluence efficiency in the beam field) and also results in maximum or near-maximum efficiency at most radii at $d_{\max }$.

\subsubsection{Performance of do_smart_brems and do_smart_compton}

As mentioned in Sections 4.3.1 and 4.3.3 above, DBS makes use of the subroutines do_smart_brems and do_smart_compton to eliminate the need for sampling and then playing Russian Roulette with split 

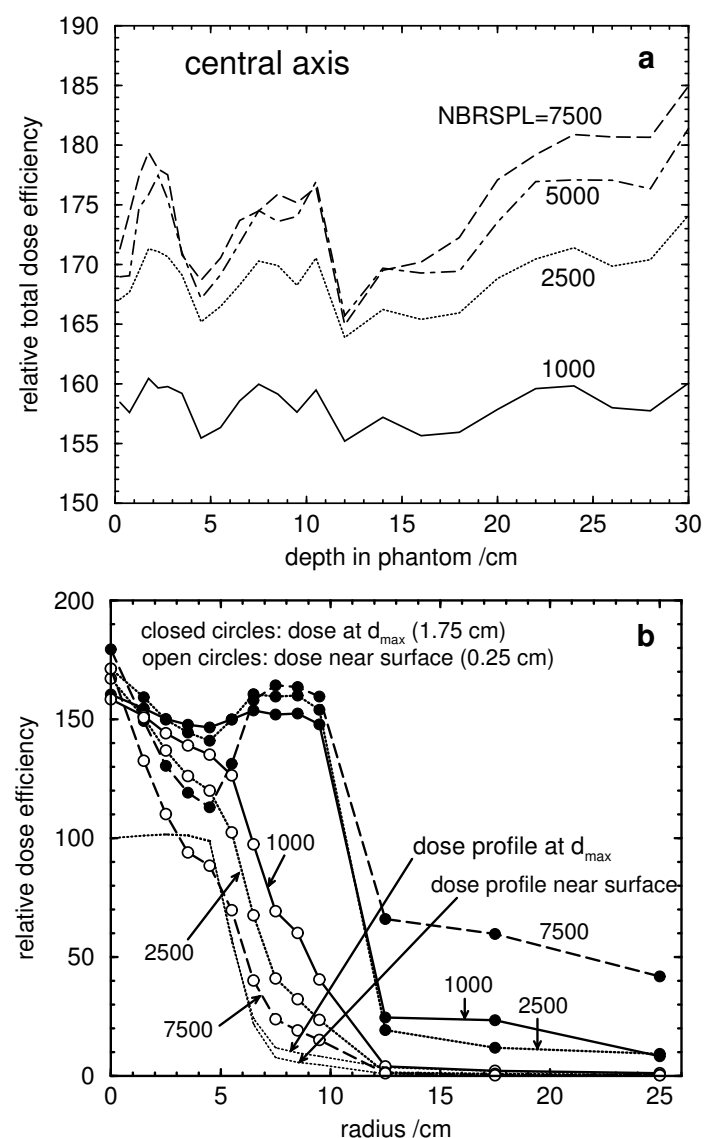

Figure 4.8: Total dose efficiency vs depth (a) and vs radius (b) in phantom for the simulated $6 \mathrm{MV}$ SL25 photon beam using DBS with different values of NBRSPL as indicated. Efficiencies have all been normalized to efficiencies with no splitting. In (b) the NBRSPL $=5000$ results (dot-dashed line) have been dropped to avoid confusion. Also in (b), efficiency vs radius results are shown at $\mathrm{d}_{\max }(1.75 \mathrm{~cm}-$ closed circles) and near the surface of the phantom ( $0.25 \mathrm{~cm}$ depth - open circles). Other DBS parameters were the same as in Figures 4.6 and 4.7.

photons not aimed into the field by only generating those photons that will be aimed into the field. In order to determine how much CPU time these subroutines actually save, we have simulated the $6 \mathrm{MV}$ SL25 photon beam using DBS (parameters optimized as described above) with all possible combinations of do_smart_brems and do_smart_compton turned on and off.

Results from this timing study are shown in Table 4.1. The top table shows the results with electron splitting off and the bottom table shows results with electron splitting on. Note that in each table, the CPU times have been normalized to the time with both do_smart_compton and do_smart_brems on (i.e. the default case).

When electron splitting is off (upper Table 4.1), it is clear that both of the smart subroutines contribute significantly to the high efficiency of DBS, with do_smart_brems playing a slightly larger role than do_- 
Table 4.1: CPU times required by simulated $6 \mathrm{MV}$ photon beam from an Elekta SL25 accelerator $(10 \times 10$ $\mathrm{cm}^{2}$ field) using DBS with all possible combinations of the time-saving subroutines do_smart_brems and do_smart_compton. CPU times are relative to CPU time using both do_smart_brems and do_smart_compton. The top table shows results with electron splitting off, and the bottom table shows results with electron splitting on.

\begin{tabular}{ll|l|l|} 
& & \multicolumn{2}{|c|}{ do_smart_brems } \\
& & ON & OFF \\
\hline \multirow{2}{*}{ do_smart_compton } & ON & 1.0 & 5.3 \\
\cline { 2 - 4 } & OFF & 3.3 & 7.6 \\
\hline
\end{tabular}

No electron splitting.

\begin{tabular}{ll|l|l|} 
& & \multicolumn{2}{|c|}{ do_smart_brems } \\
& & ON & OFF \\
\hline \multirow{2}{*}{ do_smart_compton } & ON & 1.0 & 2.43 \\
\cline { 2 - 4 } & OFF & 1.77 & 3.2 \\
\hline
\end{tabular}

Electron splitting with $\mathrm{Z}$ of splitting plane $=15.66 \mathrm{~cm}$ (back of the flattening filter) and $\mathrm{Z}$ of Russian Roulette plane $=15.5 \mathrm{~cm}$.

smart_compton. This simply indicates that when the smart routines are not used, more time is spent simulating bremsstrahlung events than simulating Compton events. It is interesting to note that the increase by a factor of 7.6 in CPU time with both subroutines off is equal to the difference in photon fluence efficiency between SBS (with Russian Roulette on and NBRSPL=1000) and DBS (with electron splitting off and NBRSPL=1000) shown in Figure 4.2.

When electron splitting is on (lower Table 4.1), the contributions of both do_smart_brems and do_smart_compton to the efficiency of DBS decrease. This is due to the fact that a much larger portion of the time in this simulation is spent tracking electrons independent of the use of do_smart_brems or do_smart_compton. Interestingly, the contribution of do_smart_compton relative to do_smart_brems is approximately the same as when electron splitting is turned off, yet electron splitting precludes the use of do_smart_compton below the Russian Roulette plane even when do_smart_compton is on. This indicates that the CPU time required for split Compton events below the Russian Roulette plane is completely overshadowed by the CPU time required for electron transport. Note that the factor of 3.2 increase in CPU time when both do_smart_compton and do_smart_brems are off is only a fraction of the factor of 8 difference in photon fluence efficiency between DBS (electron splitting on, NBRSPL=1000) and SBS (Russian Roulette off, NBRSPL=1000) shown in Figure 4.2, underscoring the fact that these subroutines play less of a role in the efficiency of DBS when electrons are generated. 


\subsection{Performance of DBS at High Energy}

To test the performance of DBS at a higher photon energy, we simulated an $18 \mathrm{MV}$ photon beam $(10 \times 10$ $\mathrm{cm}^{2}$ ) field from a Siemens KD2 accelerator and examined central-axis dose in a phantom at the SSD (100 $\mathrm{cm}$ ). Geometrical parameters were identical to those used by Sheikh-Bagheri and Rogers in their study of photon beams $[9,10]$. In addition to the various bremsstrahlung splitting routines (parameters discussed in more detail below), charged particle range rejection was used, with $\mathrm{ESAVE}=5 \mathrm{MeV}$, in all simulations.

In the case of UBS and SBS, the splitting parameters (NBRSPL and, for SBS, the splitting field size, FS) found to optimize fluence or dose efficiency in the simulated SL25 6 MV photon beam above were also found to optimize dose efficiencies in the KD2 $18 \mathrm{MV}$ simulation. Thus, for UBS NBRSPL was set to 250, and for SBS NBRSPL was set to 1000 and FS was set to $30 \mathrm{~cm}$. In the case of DBS, the positions of the electron splitting and Russian Roulette planes had to be changed to reflect the geometry and position of the flattening filter in the KD2 $18 \mathrm{MV}$ accelerator. As in the SL25 accelerator, the highest efficiencies were obtained with the splitting plane placed right on the bottom surface of the flattening filter $(\mathrm{Z}=9.8$ $\mathrm{cm}$ ). However, some trade-off's were involved in placement of the Russian Roulette plane due to the shape of the flattening filter in the KD2. The flattening filter of the KD2 has a hollowed-out portion near the bottom (see Figure 4.9). When the Russian Roulette plane was placed slightly above this portion (at $\mathrm{Z}=8.7 \mathrm{~cm}$ ), the efficiency gain obtained using DBS was approximately equal for both the photon and charged particle components of the dose. However, when the $\mathrm{Z}$ position of the Russian Roulette plane was increased to $9 \mathrm{~cm}$ (now cutting through the hollowed out portion of the flattening filter), the efficiency of the photon portion of the dose increased by $45 \%$, while the efficiency of the charged particle portion decreased by up to $40 \%$. Since the photon portion of the dose dominates the total dose at every depth, with the charged particle portion only making significant contributions in the first 5 $\mathrm{cm}$ of depth, we opted for the lower $(\mathrm{Z}=9 \mathrm{~cm})$ placement of the Russian Roulette plane. In addition, the bremsstrahlung splitting number of 1000 found to optimize dose efficiency at $6 \mathrm{MV}$ in the SL25 accelerator did not optimize central-axis dose efficiency in the KD2. By decreasing NBRSPL to 750, we were able to obtain a $14 \%$ increase in efficiency over that obtained with NBRSPL $=1000$.

Figure 4.10 shows the dose efficiencies on the central axis of the phantom (voxel radius $=1 \mathrm{~cm}$, voxel thickness $=1 \mathrm{~cm}$ or $2 \mathrm{~cm}$ ) placed at $S S D=100 \mathrm{~cm}$ in the $18 \mathrm{MV} \mathrm{KD} 2$ photon beam using the splitting parameters discussed above. The total dose efficiency shown in Figure 4.10(a) is broken down into the efficiencies of the photon and charged particle components in Figure 4.10(b). The corresponding depth-dose curves are also shown for reference. All efficiencies are relative to efficiency with no splitting. Efficiencies for the DBS case do not include any fat photons.

Figure 4.10(a) shows that DBS increased the efficiency in dose calculations on the central axis by a factor of 3.6 over SBS and by a factor of 13 over UBS. Although still offering substantial improvement over the other splitting routines, efficiency with DBS relative to no splitting dropped by a factor 2.5 from its performance in the $6 \mathrm{MV}$ SL25 beam. By comparison, relative efficiency with SBS is a factor of 1.5 lower than in the $6 \mathrm{MV}$ case, and UBS drops by a factor of only 1.3. One reason for the relative decrease in efficiency of SBS and DBS is that at higher energies the angular distribution of bremsstrahlung and 


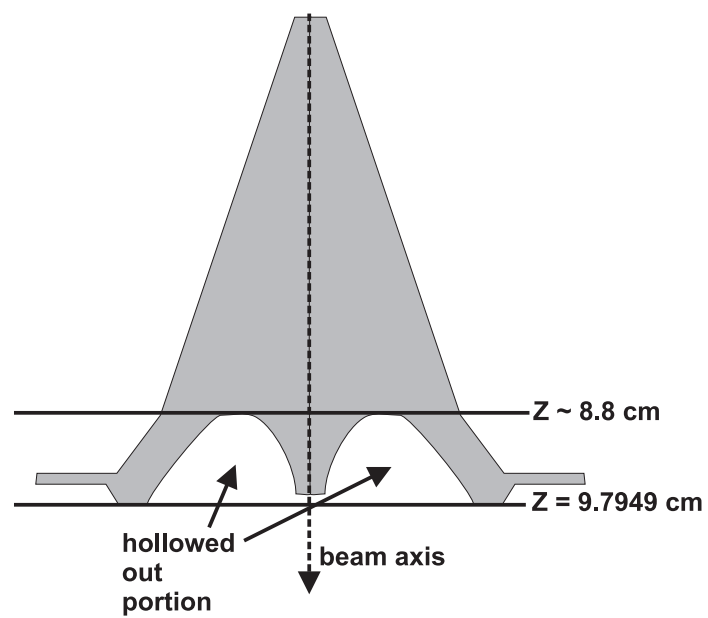

Figure 4.9: Cross-section through the flattening filter in the 18 MV KD2 photon beam showing the $\mathrm{Z}$ positions of the back of the flattening filter and the $\mathrm{Z}$ position where the hollowed out portion of the filter begins. The flattening filter is made of stainless steel.

Compton scattered photons becomes more forward peaked. In the case of SBS, this means that the splitting number will be very high for photons directed into the splitting field. For DBS, the implication is that fewer photons can be eliminated as being aimed away from the splitting field.

Another way to look at these results is to note that the overall efficiency of the BEAMnrc calculation with no splitting increases by a factor of about 2.7 going from $6 \mathrm{MV}$ to $18 \mathrm{MV}$. This is because of the more forward peaked photons at the higher energy. This means that the overall efficiency of the DBS algorithm does not change much between the $6 \mathrm{MV}$ and $18 \mathrm{MV}$ cases because it has been optimized for the forward-going photons.

In addition, the total dose efficiency with both SBS and DBS drops off near the surface of the phantom. Figure 4.10(b) shows that this drop-off is due to the lower efficiency in the charged particle contribution to the dose relative to the efficiency of the photon component of the dose in the case of DBS and SBS (charged particles account for $12.5 \%$ of the total dose near the surface of the phantom). The reason for the lower charged particle dose efficiency in the case of DBS (a factor of up to 3 lower than the efficiency of the photon component) has been discussed above, in which we opted for a placement of the Russian Roulette plane that would optimize the photon dose efficiency at the expense of some charged particle efficiency.

\subsection{Performance of DBS in a Broad Beam}

We also tested the performance of DBS in the simulated 6 MV SL25 photon beam by examining fluence efficiency at the SSD $(100 \mathrm{~cm})$ with the jaws widened to give a $40 \times 40 \mathrm{~cm}^{2}$ field. The jaws simulated in 

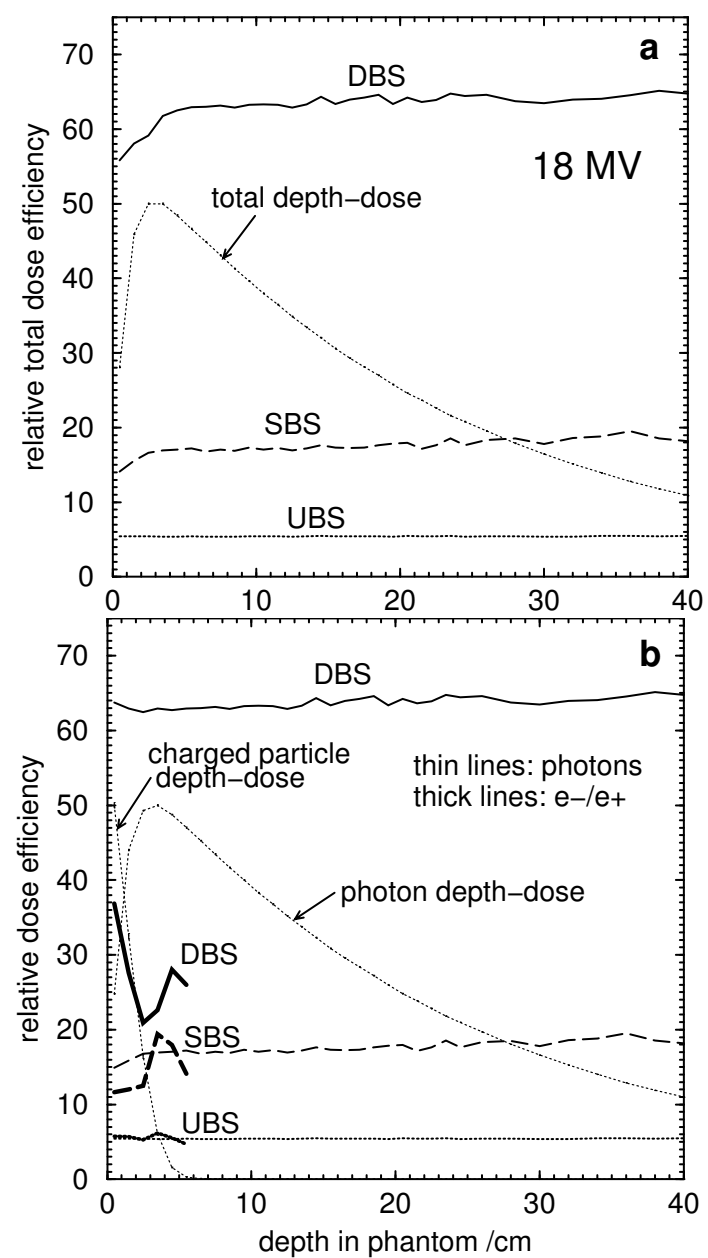

Figure 4.10: Dose efficiency vs depth in phantom for a simulated $18 \mathrm{MV}$ photon beam from a Siemens $\mathrm{KD} 2$ accelerator $\left(10 \times 10 \mathrm{~cm}^{2}\right.$ field). (a) shows total dose efficiency and (b) shows the efficiencies of the photon (thin lines) and charged particle (thick lines) components. Separately normalized depth-dose curves are shown for reference. For UBS, NBRSPL $=250$. For SBS, NBRSPL $=1000$ and $F S=30 \mathrm{~cm}$. DBS used NBRSPL $=750$, splitting radius $=10 \mathrm{~cm}$, electron splitting plane $Z=9.795 \mathrm{~cm}$ (the back of the flattening filter) and Russian Roulette plane $Z=9 \mathrm{~cm}$. For DBS, total dose efficiency shown in (a) excludes fat photons.

this case were the "standard" jaws (10 cm thick, comprising $5 \mathrm{~cm}$ of tungsten and $5 \mathrm{~cm}$ of lead) instead of the jaws for use with a multi-leaf collimator that were used to determine performance of DBS with a $10 \times 10 \mathrm{~cm}^{2}$ field in this accelerator (see section 4.4 above). Other than the jaws and their settings, the simulation geometry and other parameters were identical to those used with the $10 \times 10 \mathrm{~cm}^{2}$ field.

The splitting parameters used for all splitting routines were those found to optimize performance in the $6 \mathrm{MV}$ SL25 accelerator with a $10 \times 10 \mathrm{~cm}^{2}$ field (see section 4.4 .2 above), with the exception of the splitting field size, FS, in SBS and the splitting field radius in DBS. For SBS, FS was set to $60 \mathrm{~cm}$. This 
setting is based on the performance of SBS as a function of FS in the $10 \times 10 \mathrm{~cm}^{2}$ beam (see section 4.4.2.2 above), where little improvement in photon fluence efficiency was observed for values of FS $>$ field size $+20 \mathrm{~cm}$. The splitting radius used in DBS was $30 \mathrm{~cm}$. This radius completely encloses the $40 \times 40 \mathrm{~cm}^{2}$ field, allowing for $2 \mathrm{~cm}$ beyond the corners of the field. Note that with such a large splitting radius, the difference in efficiency with a small change in the splitting radius (eg reducing it by $2 \mathrm{~cm}$ so that it exactly encloses the field) is expected to be negligible.

For the purposes of scoring fluence, the phase-space surface at $S S D=100 \mathrm{~cm}$ was divided into 6561 (81 $\times 81) 1 \times 1 \mathrm{~cm}^{2}$ scoring zones. As with the study of photon fluence efficiency in the $10 \times 10 \mathrm{~cm}^{2}$ beam, efficiency of all splitting algorithms is expected to increase as the area of the scoring zones is decreased (see section 4.4.1 above). Unlike the $10 \times 10 \mathrm{~cm}^{2}$ field case, the $1 \times 1 \mathrm{~cm}^{2}$ scoring zones were used for both photon and electron fluence efficiency profiles.

Figure 4.11 shows the photon (a) and electron (b) fluence efficiency profiles (efficiency vs $\mathrm{X}$ at $\mathrm{Y}=0$ ) at $\mathrm{SSD}=100 \mathrm{~cm}$ in the $40 \times 40 \mathrm{~cm}^{2}$ beam. Efficiencies are relative to efficiency with no splitting. Photon and electron fluence profiles are also shown for reference. The fluctuations in relative electron fluence efficiency with DBS visible in Figure 4.11b are due mainly to fluctuations in efficiency with no splitting (i.e. the normalizing quantity) ultimately caused by the small scoring zones.

It is clear from the figures that, in the broad beam, DBS still offers a substantial improvement in efficiency over the other splitting routines. In the case of photon fluence within the field $(-20 \mathrm{~cm} \leq \mathrm{X}$ $\leq 20 \mathrm{~cm}$ ), DBS is between 5.5 (at the centre of the field) and 7 (at the edges of the field) times more efficient than SBS and is $\approx 12$ times more efficient than UBS. Between the edges of the field and the edge of the splitting field $(20 \mathrm{~cm} \leq|\mathrm{X}| \leq 30 \mathrm{~cm})$, the relative photon efficiency with DBS increases, resulting in the "horns" in Figure 4.11(a). This increase is due to the high uncertainty (low efficiency) in the photon fluence with no splitting in this region. In the case of electron fluence, the efficiency using DBS is $\approx 8$ times greater than with SBS and $\approx 14$ times greater than with UBS in the field.

The efficiency of DBS in the broad beam is significantly lower than in the $10 \times 10 \mathrm{~cm}^{2}$ beam (Figure 4.6), with photon fluence efficiency inside the field dropping by a factor of $\approx 1.7$ and electron fluence efficiency inside the field dropping by a similar amount. In comparison, photon fluence efficiency inside the field using SBS drops by a factor of only 1.1 (at the centre of the field) to 1.5 (at the edges of the field) in the broad beam, with electron fluence efficiency dropping by a factor of only $\approx 1.2$. In the case of UBS, the drop in photon and electron fluence efficiency in the broad beam compared to the $10 \times 10 \mathrm{~cm}^{2}$ beam is insignificant.

The directional splitting routines (SBS and DBS) are less efficient in the broad beam simply because of the required increase in splitting field size. In the case of DBS, this results in both fewer photons being eliminated by Russian Roulette and more photons being generated by the do_smart_brems and do_smart_compton subroutines. In the case of SBS, this results in a higher splitting number over a greater range of incident electron directions/energies. The reason that the overall efficiency drop in the broad beam is relatively greater for DBS than for SBS may be due to the increased number of split Compton interactions in DBS (SBS does not split these interactions). In the case of UBS, the change in field size does not change the number of split photons that must be tracked, resulting in no significant efficiency 

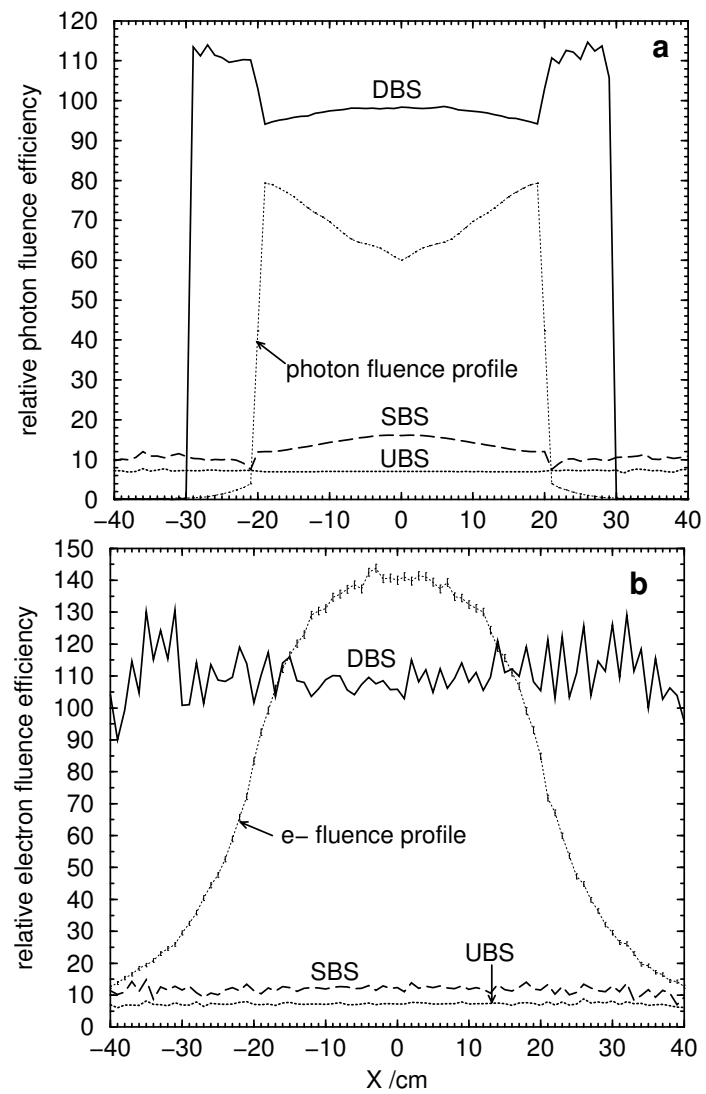

Figure 4.11: Photon (a) and electron (b) fluence efficiency vs $X($ at $Y=0)$ at the SSD $(100 \mathrm{~cm})$ for a simulated 6 MV SL25 photon beam with jaws expanded to give a $40 \times 40 \mathrm{~cm}^{2}$ field. Efficiencies are relative to the efficiency with no splitting. The arbitrarily normalized photon and electron fluence profiles at $\mathrm{Y}=0$ are also shown for comparison. For UBS, NBRSPL was set to 250. For SBS, NBRSPL $=1000$ and the splitting field size, FS, was set to $60 \mathrm{~cm}$. For DBS, NBRSPL $=1000$, splitting field radius was $30 \mathrm{~cm}, \mathrm{Z}$ of the electron splitting plane was $15.66 \mathrm{~cm}$ (the back of the flattening filter), and $\mathrm{Z}$ of the Russian Roulette plane was $15.5 \mathrm{~cm}$.

change.

It is interesting to note that for a given splitting routine in both broad beam and $10 \times 10 \mathrm{~cm}^{2}$ cases, the relative electron fluence efficiency is of the same order as the relative photon fluence efficiency which is useful since electron contamination plays a more important role in the broad beams.

\subsection{Conclusions}

We have demonstrated that directional bremsstrahlung splitting (DBS) offers a significant improvement in photon and electron fluence and dose efficiency over the previously-available bremsstrahlung splitting 
routines in BEAMnrc, uniform

bremsstrahlung splitting (UBS) and selective bremsstrahlung splitting (SBS). In a "realistic" simulation of a $6 \mathrm{MV}$ photon beam from an Elekta SL25 accelerator $\left(10 \times 10 \mathrm{~cm}^{2}\right.$ field $)$ in which photons and electrons were generated (generation of electrons entailed turning Russian Roulette off in UBS and SBS and using electron splitting in DBS), the photon fluence efficiency inside the field when using DBS was a factor of 8 higher than when using SBS. Electron fluence efficiency in the field was a factor of almost 9 higher with DBS than with SBS. Efficiency of central-axis depth-dose in a phantom placed at the SSD $(100 \mathrm{~cm})$ was over 6 times higher with DBS than with SBS.

Obtaining the optimum efficiency gain with DBS required us to optimize settings of the bremsstrahlung splitting number, NBRSPL, the splitting field radius, and the positions of the electron splitting plane and Russian Roulette plane. For the $6 \mathrm{MV}$ photon beam, we found that NBRSPL $=1000$ optimized the photon and electron fluence efficiency in the field. Since photon fluence efficiency decreased with increasing splitting field radius, it was important to choose the smallest radius that completely enclosed the field with some overlap to ensure that the contribution to central-axis dose from fat photons (photons coming back to the central axis from beyond the edge of the splitting field) was negligible. We found that a splitting field radius of $10 \mathrm{~cm}$ was sufficient to meet these requirements. Another consideration was the positions of the electron splitting and Russian Roulette planes. For a fixed splitting plane location, the efficiency could be varied by up to $10 \%$ by moving the Russian Roulette plane (Russian Roulette plane always above the splitting plane), with a trend towards higher efficiencies as both the splitting plane and Russian Roulette plane were brought closer to the bottom of the flattening filter.

The optimal settings for NBRSPL, splitting field radius and splitting and Russian Roulette plane positions will depend on the details of the accelerator being simulated. For example, the optimal setting of NBRSPL in our simulations of an $18 \mathrm{MV}$ photon beam from a Siemens KD2 accelerator was 750. Also, the hollowed out portion of the flattening filter in this accelerator was a consideration in the optimal placement of the Russian Roulette plane in relation to the electron splitting plane. However, we can generalize and say that setting NBRSPL $\simeq 1000$ will result in near-optimum performance, with adjustments around this number possibly increasing efficiency by $15 \%$. It is also a general rule that the electron splitting plane should be placed at the back of the flattening filter with the Russian Roulette plane in a solid portion of the flattening filter somewhere above the splitting plane.

The fluence or dose efficiency improvement of DBS is also dependent on the particular accelerator being simulated, with relative efficiency improvements tending to decrease at higher photon energies. This is because the inherent efficiency of these simulations is higher since bremsstrahlung photons at higher energies are more forward-directed, so fewer are subject to Russian Roulette by the DBS splitting routine. In this study, we have shown that the improvement in central-axis dose efficiency that DBS has over SBS drops from a factor of 6 in the 6 MV SL25 accelerator to a factor of 3.6 in the 18 MV KD2 accelerator.

The relative efficiency of DBS also decreases with increasing field size. When the field size of the 6 MV SL25 photon beam was increased to $40 \times 40 \mathrm{~cm}^{2}$ (broad beam), the photon and electron fluence efficiencies in the field with DBS decreased by a factor of $\approx 1.7$ from their values in the $10 \times 10 \mathrm{~cm}^{2}$ beam. 
This compared to a drop in SBS efficiency by a factor of 1.1 (centre of field) to 1.5 (edges of field) for photon fluence and by a factor of $\approx 1.2$ for electron fluence. Both SBS and DBS efficiencies were expected to drop due to the greater number of split photons that must be tracked in the broad beam, but the relatively greater efficiency decrease in DBS efficiency may be due to the greater number of split Compton events in the broad beam. Even so, DBS is still significantly more efficient than SBS in the broad beam, with photon fluence efficiency (inside the field) between 5.5 and 7 times greater than with SBS $(\approx 12$ times greater than with UBS), and electron fluence efficiency $\approx 8$ times greater than with SBS ( $\approx 12$ times greater than with UBS).

Overall, the efficiency improvement of DBS is substantial and it will save large amounts of CPU time in the simulation of photon beams.

\section{Acknowledgements}

We wish to acknowledge Daryoush Sheikh-Bagheri's contribution of the SBS algorithm in BEAMnrc and to thank Michel Proulx for his continued support of the IRS computing cluster.

\section{Appendix A: do_smart_brems algorithm}

Consider an electron (or positron) at position $\vec{x}=(x, y, z)$ travelling along the direction $\vec{u}=(u, v, w)$ that is about to undergo a bremsstrahlung event. The process is in a co-ordinate system where the beam axis is along the $z$-axis and the upper plane of the photon target is at $z=0$. The circle of interest (COI, also denoted as field of interest in the main text) is in a plane perpendicular to the $\mathrm{z}$-axis and located at $\vec{x}_{\mathrm{o}}=(\mathrm{o}, \mathrm{o}, d)(d$ is typically $100 \mathrm{~cm})$ and has a radius of $R$ (see figure 4.12$)$. We assume that the angular distribution $p(\mu, \phi)$ of bremsstrahlung photons is described by the leading term of Eq. $2 \mathrm{BS}$ from the article by Koch and Motz[12]:

$$
p(\mu, \phi)=\frac{1-\beta^{2}}{4 \pi} \frac{1}{(1-\beta \mu)^{2}} .
$$

Here, $\mu$ is the cosine of the polar scattering angle (i.e. $\cos (\theta)), \phi$ is the azimuthal angle and $\beta$ is the electron velocity in units of the speed of light. The probability given in Eq. (4.2) is normalized over all angles to unity. The goal is to calculate the probability $W$ that this electron will emit a bremsstrahlung photon that is pointed towards the circle of interest. If $W$ is known and one wants to perform bremsstrahlung splitting with a splitting number of $N$, keeping only photons going towards the COI, only $W N$ photons need to be sampled provided that their angles are sampled so that they are directed towards the COI. If $W \ll 1$, a substantial saving of CPU time may be achieved.

The probability $W$ is given by

$$
W=\int_{\Omega} \mathrm{d} \mu \mathrm{d} \phi p(\mu, \phi)
$$

where the integration is to be carried out for all $\mu, \phi$ within the solid angle $\Omega$ that result in a direction 


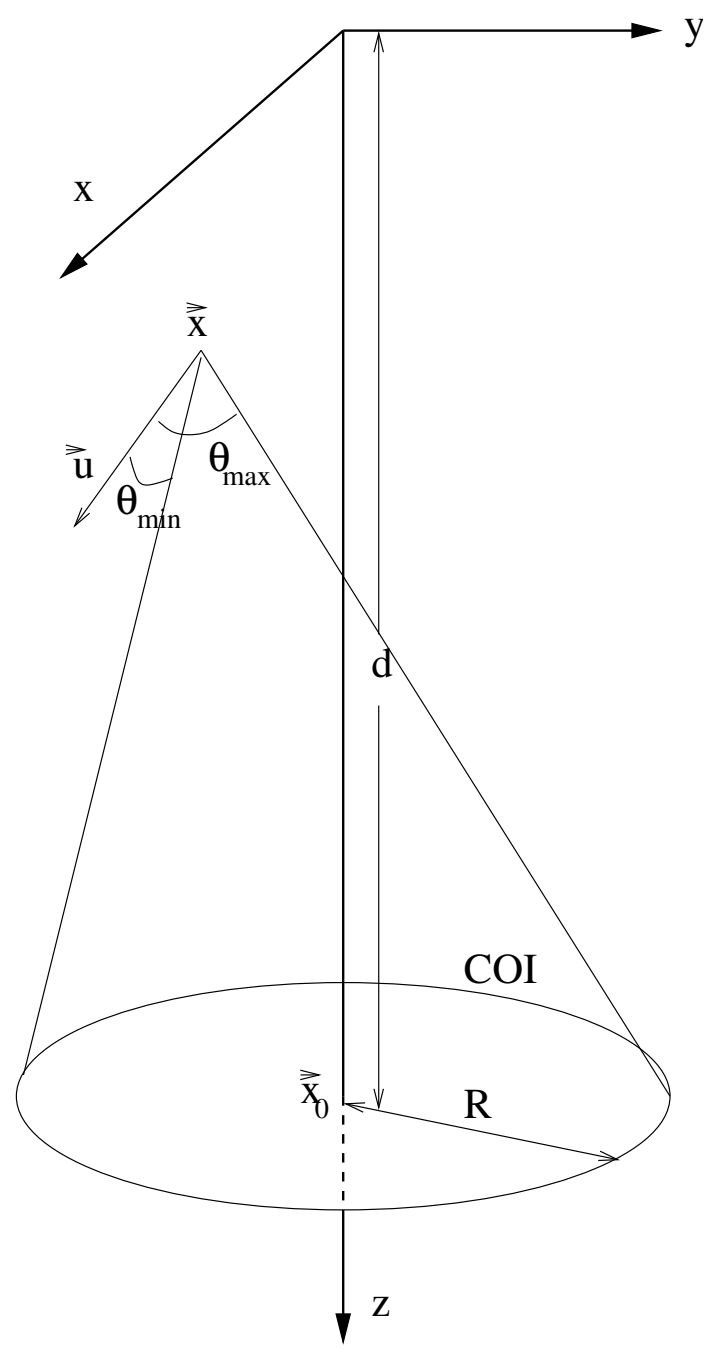

Figure 4.12: The geometry used to derive the equations in Appendix A and B. Note that $\mu_{\max }=\cos \left(\theta_{\max }\right)$, etc.

towards the COI. With the definitions

$$
\begin{aligned}
& r=\sqrt{x^{2}+y^{2}} \\
& a_{ \pm}=\vec{u}\left(\vec{x}_{\circ}-\vec{x}\right) \pm R \sqrt{1-w^{2}} \\
& t_{\max }=\sqrt{d^{2}+(R+r)^{2}} \\
& t_{\text {min }}= \begin{cases}\sqrt{d^{2}+(R-r)^{2}}, & r \leq R \\
d & , \quad r>R\end{cases}
\end{aligned}
$$


and simple geometrical considerations it is easy to see that the minimum and maximum polar scattering angles, $\mu_{\min }$ and $\mu_{\max }$, that may result in a direction towards the COI are given by

$$
\begin{aligned}
& \mu_{\max }=\operatorname{Min}\left(1, \mu_{+}\right), \quad \mu_{+}= \begin{cases}a_{+} / t_{\min }, & a_{+} \geq 0 \\
a_{+} / t_{\max }, & a_{+}<0\end{cases} \\
& \mu_{\min }=\operatorname{Max}\left(-1, \mu_{-}\right), \quad \mu_{-}= \begin{cases}a_{-} / t_{\max }, & a_{-} \geq 0 \\
a_{-} / t_{\min }, & a_{-}<0\end{cases}
\end{aligned}
$$

The possible range of azimuthal scattering angles depends on $\vec{x}, \vec{x}_{\circ}$ and $\vec{u}$ in a complicated way. In addition, if the fact is taken into account that not all azimuthal angles will lead to a direction towards the COI, the integration in Eq. (4.3) can not be performed analytically. However, we can provide an upper limit of the probability $W$, denoted by $W^{\prime}$, by using all azimuthal angles. The correct number of photons towards the COI will result by simulating $W^{\prime} N$ bremsstrahlung events and then rejecting photons not going towards the COI. We have

$$
W^{\prime}=2 \pi \int_{\mu_{\min }}^{\mu_{\max }} \mathrm{d} \mu p(\mu)=\frac{\left(1-\beta^{2}\right)\left(\mu_{\max }-\mu_{\min }\right)}{2\left(1-\beta \mu_{\max }\right)\left(1-\beta \mu_{\min }\right)}
$$

with $\mu_{\min }$ and $\mu_{\max }$ defined via Eq. (4.4) and (4.5).

The algorithm of the do_smart_brems subroutine is then as follows:

1. Calculate $\mu_{\min }, \mu_{\max }$ and the estimated probability $W^{\prime}$.

2. Determine the number of photon angles, $N^{\prime}$, to be sampled. If we denote by $[\alpha]$ the integer part of $\alpha$ and by $\eta$ a random number uniformly distributed between zero and unity, then $N^{\prime}=\left[W^{\prime} N\right]+1$, if $\eta \leq W^{\prime} N-\left[W^{\prime} N\right], N^{\prime}=\left[W^{\prime} N\right]$, otherwise.

3. Sample $N^{\prime}$ polar angles $\mu_{i}$ between $\mu_{\min }$ and $\mu_{\max }$ from the probability distribution $p(\mu)$ and azimuthal angles uniformly between 0 and $2 \pi$. Sampling $\mu_{i}$ is accomplished using

$$
\mu_{i}=\frac{\mu_{\min }\left(1-\beta \mu_{\max }\right)+\eta_{i}\left(\mu_{\max }-\mu_{\min }\right)}{1-\beta \mu_{\max }+\beta \eta_{i}\left(\mu_{\max }-\mu_{\min }\right)}
$$

where $\eta_{i}$ is a random number uniformly distributed between zero and unity.

4. Reject all photons that do not go towards the COI. This will lead to a smaller number of photons $N^{\prime \prime}$, all of them having a weight of $1 / N$.

5. Sample a polar angle from $p(\mu)$ between -1 and 1 . If this angle is not between $\mu_{\min }$ and $\mu_{\max }$, keep this photon with a weight of 1 and increase $N^{\prime \prime}$ by one.

6. Sample $N^{\prime \prime}$ photon energies from the bremsstrahlung cross section differential in energy. If $N^{\prime \prime}=$ o, sample one photon energy.

7. Decrease the electron energy by the energy of the last sampled photon energy. 


\section{Appendix B: do_smart_compt algorithm}

Consider a photon with energy $k$, position $\vec{x}$ and direction $\vec{u}$ that is about to undergo a Compton scattering event modelled using the Klein-Nishina cross section [13]. Within the Klein-Nishina approximation each polar scattering angle $\mu=\cos (\theta)$ uniquely corresponds to a scattered photon energy $k^{\prime}$,

$$
k^{\prime}=\frac{k}{1+k(1-\mu)}
$$

where for the simplicity of the notation all energies are expressed in terms of the electron rest energy. As discussed in Appendix A, the minimum and maximum angles, $\mu_{\min }$ and $\mu_{\max }$, that may result in a scattered photon going towards the circle of interest (COI) are given by Eq. (4.5). The corresponding minimum and maximum scattered photon energies, $k_{\min }^{\prime}$ and $k_{\max }^{\prime}$ are given by Eq. (4.8) with $\mu=\mu_{\min }$ or $\mu=\mu_{\max }$, respectively. The upper bound of the probability $W$ for having the scattered photon moving towards the COI, $W^{\prime}$ (see Appendix A for discussion of why we must use $W^{\prime}$ instead of $W$ ), is

$$
W^{\prime}=\frac{\int_{k_{\min }^{\prime}}^{k_{\max }^{\prime}} \mathrm{d} k^{\prime} \mathrm{d} \sigma_{\mathrm{KN}} / \mathrm{d} k^{\prime}}{\int_{k /(1+2 k)}^{1} \mathrm{~d} k^{\prime} \mathrm{d} \sigma_{\mathrm{KN}} / \mathrm{d} k^{\prime}}=\frac{H\left(k_{\min }, k_{\max }\right)}{H(1 /(1+2 k), 1)}
$$

where $\mathrm{d} \sigma_{\mathrm{KN}} / \mathrm{d} k^{\prime}$ is the Klein-Nishina cross section and

$$
H\left(x_{1}, x_{2}\right)=\ln \frac{x_{2}}{x_{1}}\left(k^{2}-2 k-2\right)+\left(x_{2}-x_{1}\right)\left(\frac{k}{x_{1} x_{2}}+\frac{1+2 k}{k}+\frac{k}{2}\left(x_{1}+x_{2}\right)\right) .
$$

The algorithm of the do_smart_compt subroutine is then as follows:

1. Calculate $\mu_{\min }, \mu_{\max }$ and the probability $W^{\prime}$.

2. Determine the number $N^{\prime}$ of Compton interactions to be sampled. If we denote by $[\alpha]$ the integer part of $\alpha$ and by $\eta$ a random number uniformly distributed between zero and unity, then $N^{\prime}=$ $\left[W^{\prime} N\right]+1$, if $\eta \leq W^{\prime} N-\left[W^{\prime} N\right], N^{\prime}=\left[W^{\prime} N\right]$, otherwise.

3. Sample $N^{\prime}$ scattered photon energies between $k_{\min }^{\prime}$ and $k_{\max }^{\prime}$ from the Klein-Nishina cross section. The algorithm is very similar to the one used to sample the full distribution (see e.g. the EGSnrc manual [7]), except that the constants $\alpha_{1}$ and $\alpha_{2}$ have to be calculated using $k_{\min }^{\prime}$ and $k_{\max }^{\prime}$ instead of $k /(1+2 k)$ and $k$ as the integration limits.

4. Calculate the corresponding scattering angles and photon directions and reject all photons not moving towards the COI. Also reject all electrons.

5. Sample one Compton interaction without restrictions. Keep the resulting electron with a weight of 1 . Keep the scattered photon only if its energy is not between $k_{\min }^{\prime}$ and $k_{\max }^{\prime}$ and give it the weight of 1 . 


\subsection{Bibliography}

[1] D. W. O. Rogers, B. A. Faddegon, G. X. Ding, C.-M. Ma, J. Wei, and T. R. Mackie. BEAM: A Monte Carlo code to simulate radiotherapy treatment units. Med. Phys., 22:503 - 524, 1995.

[2] D. W. O. Rogers, C.-M. Ma, G. X. Ding, B. Walters, D. Sheikh-Bagheri, and G. G. Zhang. BEAMnrc Users Manual. NRC Report PIRS 509(a)revF, 2001.

[3] D. W. O. Rogers. Publications using or about BEAM or the OMEGA project . http://www.irs.inms.nrc.ca/inms/irs/BEAM/bibliog/omega_pubs.html.

[4] D. W. O. Rogers, C.-M. Ma, G. X. Ding, B. Walters, D. Sheikh-Bagheri, and G. G. Zhang. BEAM98 Users Manual. NRC Report PIRS 509(a)revC, 1998.

[5] D. Sheikh-Bagheri. Monte Carlo study of photon beams from medical linear accelerators; optimization, benchmark and spectra. PhD. thesis, Carleton University, Ottawa, 1999.

[6] I. Kawrakow. Accurate condensed history Monte Carlo simulation of electron transport. I. EGSnrc, the new EGS4 version. Med. Phys., 27:485 - 498, 2000.

[7] I. Kawrakow and D. W. O. Rogers. The EGSnrc Code System: Monte Carlo simulation of electron and photon transport. Technical Report PIRS-701, National Research Council of Canada, Ottawa, Canada, 2000.

[8] I. Lux and L. Koblinger. Monte Carlo particle transport methods: neutron and photon calculations. CRC Press, New York, 1991.

[9] D. Sheikh-Bagheri and D. W. O. Rogers. Sensitivity of megavoltage photon beam Monte Carlo simulations to electron beam and other parameters. Med. Phys., 29:379 - 390, 2002.

[10] D. Sheikh-Bagheri and D. W. O. Rogers. Calculation of nine megavoltage photon beam spectra using the BEAM Monte Carlo code. Med. Phys., 29:391 - 402, 2002.

[11] B. R. B. Walters, I. Kawrakow, and D. W. O. Rogers. History by history statistical estimators in the BEAM code system. Med. Phys., 29:2745 - 2752, 2002.

[12] H. W. Koch and J. W. Motz. Bremsstrahlung cross-section formulas and related data. Rev. Mod. Phys., 31:920 - 955, 1959.

[13] O. Klein and Y. Nishina. Über die Streuung von Strahlung durch freie Elektronen nach der neuen relativistischen Quantendynamik von Dirac. Z. für Physik, 52:853-868, 1929. 



\section{Increasing efficiency of BEAMnrc-simulated Co-60 beams using directional source biasing}




\begin{abstract}
Purpose: This study describes the implementation of a directional source biasing (DSB) scheme for efficiently simulating Cobalt-60 treatment heads using the BEAMnrc Monte Carlo code. Previous simulation of Co- 60 beams with BEAMnrc were impractical because of the time required to track photons not directed into the treatment field as well as secondary charged particles.

Methods: In DSB, efficiency is increased by splitting each photon emitted by the Co60 source a user-defined number of times. Only those split primary photons directed into a user-defined splitting field (encompassing the treatment field) are sampled, yielding many low-weight photons directed into the field. Efficiency can be further increased by taking advantage of radial symmetry at the top of the treatment head to reduce the number of split primary photons tracked in this portion. There is also an option to generate contaminant electrons in DSB.
\end{abstract}

Results: The DSB scheme in BEAMnrc increases the photon fluence calculation efficiency in a $10 \times 10 \mathrm{~cm}^{2}$ Co-60 beam by a factor of 1,800 with a concurrent increase in contaminant electron fluence calculation efficiency by a factor of 1,200 . Implementation of DSB in beampp, a C++ code for accelerator simulations based on EGSnrc and the $\mathrm{C}++$ class library, egspp, increases photon fluence efficiency by a factor of 2,800 and contaminant electron fluence efficiency by a factor of 1,600. Optimum splitting numbers are in the range $20,000-40,000$. For dose calculations in a water phantom $\left(0.5 \times 0.5 \times 0.5 \mathrm{~cm}^{3}\right.$ voxels $)$ this translates into an increased dose calculation efficiency (all doses $>0.5 \times \mathrm{D}_{\max }$ ) of a factor of $\sim 400$. An example calculation of the ratio of dose to water to dose to chamber (the basis of the beam quality correction factor) to within $0.2 \%$ in a realistic chamber using a full simulation of a Co-60 treatment head as a source indicates the practicality of Co-60 simulations with DSB.

Conclusions: The efficiency improvement resulting from DSB makes Monte Carlo commissioning of Co-60 beams and calculation of beam quality and correction factors feasible. 


\subsection{Introduction}

Ongoing interest in the Monte Carlo modeling of Cobalt- 60 treatment beams stems from the possibility of accurately calculating correction factors[1,2], commissioning Co-60 beams for use in IMRT[3-5] and using a Co-60 beam together with magnetic resonance imaging (MRI) in image guided radiotherapy (IGRT)[4-7].

BEAMnrc[8], a powerful tool for simulating medical accelerators based on the EGSnrc Monte Carlo package[9], has been used by Mora et al[10] to accurately characterize Co-60 beams, however simulation times to achieve precise results are too long to be of practical use. For example, using Mora et al's simulation parameters for a $10 \times 10 \mathrm{~cm}^{2}$ beam incident on a $21 \mathrm{~cm} \times 21 \mathrm{~cm} \times 30 \mathrm{~cm}$ water phantom $(0.5 \mathrm{~cm} \times$ $0.5 \mathrm{~cm} \times 0.5 \mathrm{~cm}$ voxels) requires over $1,500 \mathrm{~h}$ on a $1.8 \mathrm{GHz}$ Opteron $244 \mathrm{CPU}$ to obtain $0.1 \%$ uncertainty on doses $>0.5 \times \mathrm{D}_{\max }$.

The inefficiency of Co-60 simulations is due to the fact that much of the calculation time is spent tracking photons from the Co-60 source not directed into the treatment field and secondary electrons. This work describes a new directional source biasing (DSB) algorithm which addresses these issues by: 1) isotropically splitting each photon emitted by the Co-60 source (primary photon) and sampling only those split primary photons that are directed into a user-defined splitting field (encompassing the treatment field), 2) using the radial symmetry of the Co-60 treatment head above the primary collimator (usually pyramidal) to reduce the number of primary photons tracked above the collimator, and 3) reducing the number of secondary electrons tracked by subjecting them to Russian Roulette. If the user is interested in contaminant electron effects, then contaminant electrons can be regenerated by splitting high-weight electrons upon crossing a user-defined splitting plane. Electron splitting for simulating contaminant electrons in DSB uses the same algorithm as that used by directional bremsstrahlung splitting, already in place in BEAMnrc[11] for efficient photon beam calculations.

A description of the DSB algorithm is given in Section 5.2 of the paper. DSB is benchmarked by performing a voxel-by-voxel comparison of photon and electron particle fluence with and without DSB turned on. This accuracy test is described in Section V.A. The optimization of DSB with respect to photon splitting number, nsplit, and the minimum linear distance between radially redistributed photons, $\Delta$, is described in Sections V.B and V.C. A realistic chamber simulation used to demonstrate the viability of performing Monte Carlo calculations of $\mathrm{k}_{Q}$ is described in Section 5.6. Concluding remarks appear in Section 5.7 .

\subsection{The directional source biasing (DSB) algorithm}

In DSB the user defines a splitting number, nsplit, by which primary photons emitted from the Co-60 source are split isotropically. The user also defines a splitting field radius, $\mathrm{fs}$, and a source-to-surface distance at which the splitting field is defined, ssd. The splitting field radius should at least encompass the treatment field. 
Individual rejection of split primary photons not directed into the splitting field is time consuming, and DSB samples only those photons directed into the splitting field using a variation of a method for point source biasing developed by Bielajew et al[12].

Given an isotropically radiating point source at a distance ssd directly above the centre of a circular field of radius $\mathrm{fs}$, Bielajew et al[12] show that sampling the non-uniform spatial distribution of particles incident on the field is equivalent to sampling a uniform distribution of particles over the circular field and adjusting particle weights, wt, to be:

$$
\mathrm{wt}=\frac{\pi(\mathrm{fs})^{2} \mathrm{ssd}}{\left((\mathrm{ssd})^{2}+x_{i}^{2}+y_{i}^{2}\right)^{3 / 2}}
$$

where $\mathrm{x}_{i}$ and $\mathrm{y}_{i}$ are the $\mathrm{X}$ and $\mathrm{Y}$ coordinates of the point of incidence on the circular field. Rearranging the right hand side of Equation (5.1):

$$
\begin{aligned}
\text { wt } & =\frac{\pi(\mathrm{fs})^{2}}{(\mathrm{ssd})^{2}}\left(\frac{\mathrm{ssd}}{\sqrt{(\mathrm{ssd})^{2}+x_{i}^{2}+y_{i}^{2}}}\right)^{3} \\
& =\frac{\pi(\mathrm{fs})^{2}}{(\mathrm{ssd})^{2}} \mathrm{win}^{3}
\end{aligned}
$$

where win is the $\mathrm{Z}$ direction cosine of the particle. Since $\frac{\pi(\mathrm{fs})^{2}}{(\mathrm{ssd})^{2}}$ is a constant, this can be removed, and particles are weighted by win ${ }^{3}$.

Variations in particle weight have the potential to adversely affect the statistics of Monte Carlo calculations[11], and an equivalent to generating particles with the variable weights of Equation (5.2) is to give all particles the same weight and reject those for which the following criterion is not met:

$$
\text { rnd }<\text { win }^{3}
$$

where rnd is a random number on $[0,1)$ chosen for each particle. This rejection scheme has been used for years in DOSXYZnrc[13] and EGSnrc user codes[14] for collimated point sources.

Assuming that the radial dimension of the source capsule is small compared to $\sqrt{(\mathrm{fs})^{2}+(\mathrm{ssd})^{2}}$ (i.e. the source capsule can be regarded as a point source with respect to the splitting field), the above method can be applied to DSB. For each split primary photon, nthin photons are generated with projected final positions uniformly (randomly) sampled over the splitting field, where:

$$
\text { nthin }=\frac{(f s)^{2}}{(2(s s d-z i n))^{2}} \text { nsplit }
$$

where $z$ in is the initial $Z$ position of the emitted photon. Each split photon is then subject to the rejection criterion of Equation (5.3), and surviving photons all have weight 1/nsplit. Note that Equation (5.4) is simply nsplit multiplied by the ratio of the area of the splitting field to the area of the sphere subtended by the splitting field and the point where the primary photon is emitted.

For each primary photon DSB also generates a single fat photon with a random direction sampled 
isotropically. If the photon is directed outside the splitting field it is kept. In this way primary photons directed away from the splitting field are adequately represented.

DSB deals with non-integer values of nthin by first truncating nthin and then comparing a random number on $[0,1)$ to the remainder. If the random number is less than the remainder, nthin is incremented by one. This ensures that, on average, the calculated number of incident photons is generated per primary history.

\subsubsection{Use of radial symmetry}

If the treatment head, including the source capsule, is radially symmetric about the Z-axis above a given plane (the DSB splitting plane) then computation time can be saved by using this symmetry to reduce the number of photons tracked above this plane. This is accomplished in DSB by dividing the splitting field into radial bins as shown in Figure 5.1. The number of photons directed into bin $i$ is reduced by a factor of $i$ (and their weight increased by a factor of $i$ ). Once these photons reach the splitting plane, below which radial symmetry no longer holds, the factor of $i$ is recovered by splitting the photons $i$ times (reducing their weight by a factor of $i$ ) and evenly distributing them about their radial position with respect to the Z-axis.

The split primary photons entering the splitting field are now simulated by generating nthin' photons with projected final positions in the splitting field sampled from the probability distribution function, $\mathrm{p}_{i}$, for the radial bins, where:

$$
\text { nthin }^{\prime}=\frac{(\mathrm{fs})^{2}}{(2(\mathrm{ssd}-\mathrm{zin}))^{2}}\left(\sum_{i=1}^{\text {nbin }} p_{i}\right) \text { nsplit }
$$

and:

$$
p_{i}=\frac{\left(r_{i+1}^{2}-r_{i}^{2}\right)}{(\mathrm{fs})^{2} i}
$$

with the rejection criterion of Equation (5.3). Note that $\mathrm{p}_{i}$ is simply the ratio of the area of bin $i$ to the area of the splitting field reduced by a factor of $i$ (to reflect the fact that $i$ photons will be regenerated), and nthin' is less than nthin from Equation (5.4) because $\sum_{i=1}^{\mathrm{nbin}} p_{i}<1$.

The parameter used to divide the splitting field into bins is the minimum linear distance (projected to ssd), $\Delta$, between photons that are split/redistributed at the splitting plane. The value of $\Delta$ is a user input, and a discussion of efficiency of DSB versus $\Delta$ appears in Section V.B below. The value of $\Delta$ determines the minimum radius of bin $\mathrm{i}, \mathrm{r}_{i}$ :

$$
r_{i}=\frac{\Delta}{2 \sin \left(\frac{\pi}{i}\right)}, i=2,3, \ldots, \text { nbin }
$$

Note that $r_{1}=0$ and photons directed into bin 1 are not split/redistributed.

The total number of radial bins, nbin, is equal to the number of times a photon can be split/redistributed 


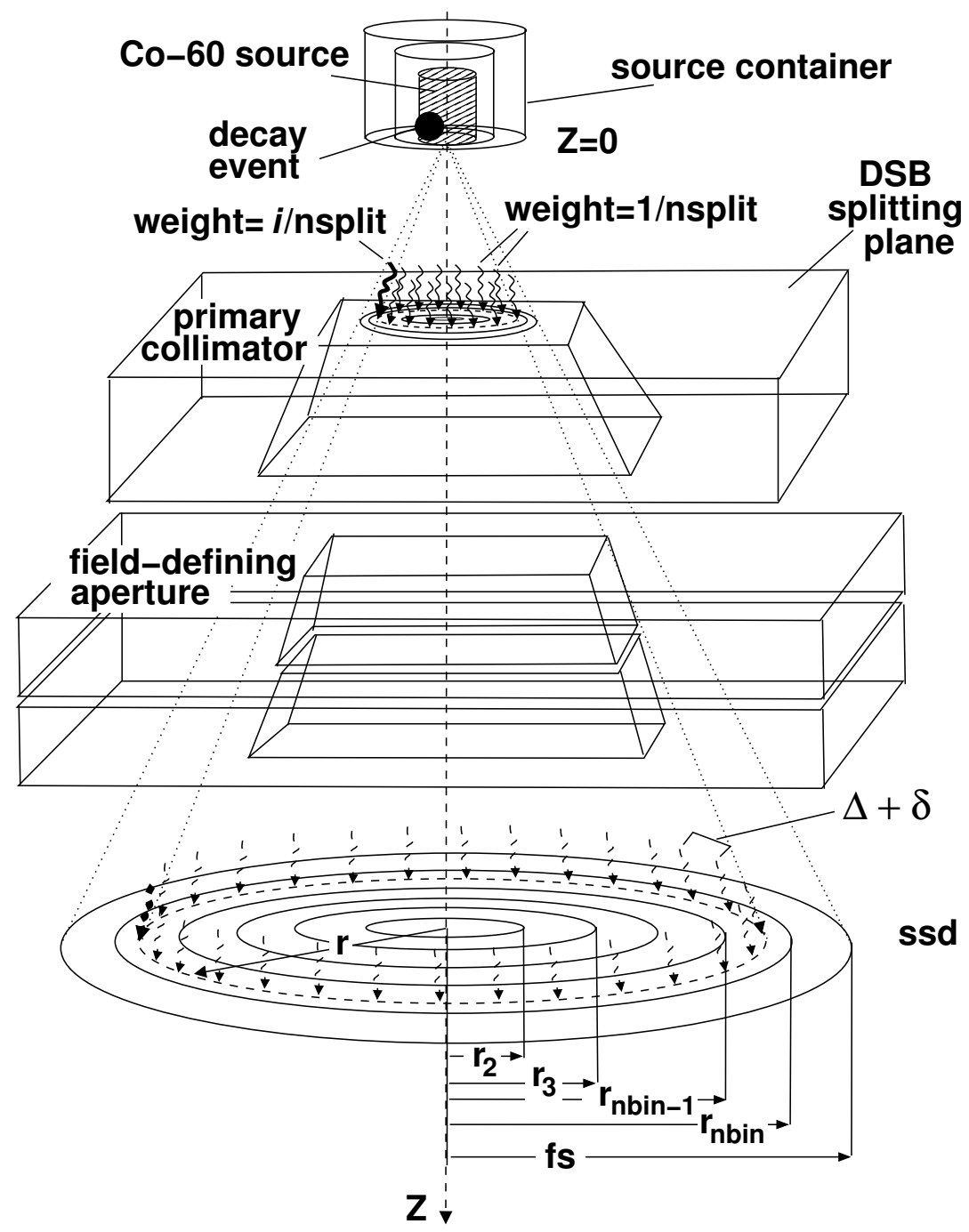

Figure 5.1: A schematic of DSB in a Co- 60 treatment head. The splitting field, defined by $f s$ and $s s d$, is divided into nbin radial bins. For each radial bin $i$, defined by minimum radius, $\mathrm{r}_{i}$, the number of split primary photons directed into the bin is reduced by a factor of $i$. Once the primary photon crosses the splitting plane, below which radial symmetry no longer holds, the factor of $i$ is recovered by splitting the photon $i$ times and redistributing the split photons about their radial position. The minimum linear distance between split/redistributed photons (projected to ssd) is $\Delta$, a user input. In general, the linear distance between split/redistributed photons is $\Delta+\delta$, where $0 \leq \delta<\Delta$. Primary photons directed into bin $i$ have weight $i /$ nsplit. Once they are split/redistributed, they have weight $=1 /$ nsplit. The schematic is based on the Eldorado Co-60 unit simulated in this study. 
at the outer edge of the splitting field, $f \mathbf{s}$, and is given by:

$$
\operatorname{nbin}=\frac{\pi}{\operatorname{asin}\left(\frac{\Delta}{2 \mathrm{fs}}\right)}-1
$$

where $\frac{\Delta}{2 \mathrm{fs}}$ is in radians. Note also that the number of times a photon is split upon reaching the DSB splitting plane cannot exceed the overall splitting number, nsplit. While this imposes a theoretical lower limit on the value of $\Delta$, in general the large value of nsplit used means that $\Delta$ is actually limited by the maximum dimension of the array storing radial bin data $\left(\mathrm{r}_{i}, \mathrm{p}_{i}\right.$, etc $)$.

\subsubsection{Treatment of higher order photons and contaminant electrons}

Above the DSB splitting plane, secondary photons produced from bremsstrahlung or other radiative events directed into the splitting field are subject to Russian Roulette with survival probability $1 / i$, where $i$ is the index of the radial bin that they are directed into. Surviving photons have their weight increased by a factor of $i$.

In general, DSB saves computation time by subjecting all secondary electrons to Russian Roulette with survival probability $i /$ nsplit (where $i$ is the index of the radial bin into which the parent photon is directed) above the DSB splitting plane and 1/nsplit below resulting in a very small number of fat (weight=1) surviving electrons. Contaminant electrons can be regenerated by turning the electron splitting option in directional bremsstrahlung splitting[11] on and specifying an electron splitting plane and a Russian Roulette plane. Non-fat electrons will no longer be subject to Russian Roulette below the Russian Roulette plane, and fat (weight=1) electrons reaching the electron splitting plane will be split nsplit times. Any radiative events undergone by non-fat charged particles, with weight $i / n s p l i t$, above the DSB splitting plane are split $i$ times, and resultant photons are subject to Russian Roulette as described in the paragraph above (Note: the radial bin into which secondary photons are directed is not necessarily the same as that of the parent photon which gave rise to the charged particle).

\subsubsection{Software considerations}

The introduction of DSB includes a redefinition of the BEAMnrc stack variable, iphat. Previously, iphat was used with directional bremsstrahlung splitting as a flag to indicate if a particle was fat (iphat=1) or thin (iphat $=0$ ). Now, however, particles above the DSB splitting plane can have varying degrees of "thinness", depending on which radial bin, it $i$, they or their parent particle is directed into. The redefined iphat takes on values $1,2, \ldots, n b i n, n s p l i t$ above the DSB splitting plane, where iphat=nsplit means the particle is fat. Thus, iphat keeps an instantaneous record of which radial bin the particle (or its parent) is directed into and allows quick computation of Russian Roulette survival probabilities (1/iphat). Note that below the DSB splitting plane iphat will be either 1 (thin) or nsplit (fat). 


\subsubsection{Augmented range rejection}

Simulations using DSB also make use of a time-saving feature called augmented range rejection (first introduced with directional bremsstrahlung splitting[11]). Charged particles that are not fat and have energy $\leq$ ESAVE are first subject to range rejection. If they are not eliminated in this way, then they are subject to Russian Roulette with survival probability iphat/nsplit. If they survive Russian Roulette, then they have their weight increased by a factor of iphat (i.e they become fat). This can save some simulation time over standard range rejection, although in the case of the Co-60 irradiators described here, the savings amount to only a few percent.

\subsubsection{Numerical example}

A measure of the number of split primary photons generated by DSB can be obtained by considering a typical Co-60 treatment head with $\mathrm{ssd}=100 \mathrm{~cm}$ and a $10 \mathrm{~cm} \times 10 \mathrm{~cm}$ treatment field. Using a splitting field radius, $f s$, of $10 \mathrm{~cm}$ and an optimum splitting number, nsplit, of 20,000 (see Section V.B below) in Equation (5.4), DSB generates approximately 50 photons directed into the splitting field per photon emitted by the source. If radial symmetry is used with $\Delta=1.5 \mathrm{~cm}$ (see Section V.B), DSB divides the splitting field into 40 radial bins with a sum of probabilities of a particle being directed into each bin, $\sum_{i=1}^{\mathrm{nbin}} p_{i}$, of $5.35 \times 10^{-2}$ (individual $\mathrm{p}_{i}$ calculated using Equation (5.6)). This reduces the number of photons per primary event to approximately 3 . This example indicates the large splitting numbers involved when using DSB and the degree of repetition of information in the radially symmetric portion of the treatment head.

\subsection{Simulations}

\subsubsection{Co-60 beam simulations}

In order to study the efficiency of DSB, Co-60 beams are simulated using both BEAMnrc and beampp. The latter uses the EGSnrc C++ class library, egspp[15], to simulate accelerators. It has much of the functionality of BEAMnrc and uses BEAMnrc input files. With both BEAMnrc and beampp, the Co-60 simulations are run as shared-library sources. Shared library sources have been shown[16] to be similar in efficiency to phase space sources, provided that variance reduction techniques are used to optimize the accelerator simulation, and have the advantage that large phase space files need not be stored.

Geometric parameters used for the Co- 60 beam simulations are the same as those employed by Mora et al[10] to simulate an Eldorado 6 unit used at the National Research Council Canada for ion chamber calibrations. The Co-60 source is modeled using BEAMnrc[8] source 3. The energy spectrum of the source is a simple two-line spectrum, with equiprobable gamma rays at 1.17 and $1.33 \mathrm{MeV}$.

To reduce their simulation times, Mora et al[10] varied the electron transport cutoff energy (ECUT) throughout the treatment head, with ECUT ranging from $0.6 \mathrm{MeV}$ in the collimator air and air gap to 1.921 
$\mathrm{MeV}$ in the lead and iron in the source container. Moreover, they employed range rejection - immediate cessation of charged particle transport if the particle cannot make it to the nearest region boundary on charged particles at all energies (i.e. the ESAVE parameter was set $>1.33 \mathrm{MeV}$ ). These time saving techniques impact bremsstrahlung yield, and it is found that they result in a $\sim 0.5 \%$ difference in peak photon fluence per primary history when compared to simulations where ECUT is set to $0.7 \mathrm{MeV}$ in all regions except the collimator air and air gaps and range rejection is only performed on charged particles with energy $\leq 0.7 \mathrm{MeV}(\mathrm{ESAVE}=0.7 \mathrm{MeV})$. Since $<0.5 \%$ precision is desired to assess potential biases in photon fluence introduced by the DSB algorithm, all simulations in this study use ECUT $=0.7 \mathrm{MeV}$ everywhere except collimator air and air gaps, where ECUT $=0.6 \mathrm{MeV}$, and range rejection with ESAVE $=0.7$ $\mathrm{MeV}$. The photon transport cutoff energy (PCUT) is $0.01 \mathrm{MeV}$.

The radial symmetry of this particular Co-60 treatment head geometry ends at the top of the primary collimator (which has square symmetry and is simulated using a PYRAMIDS component module in BEAMnrc). Thus, the DSB splitting plane, where photons are split and redistributed evenly about their radial position, as described in Section 5.2 above, is placed at the top of the primary collimator geometry $(\mathrm{Z}=1.5 \mathrm{~cm})$ in all simulations using DSB.

In order to include contaminant electrons in the beam, Co-60 simulations with DSB are run with the electron splitting option turned on. Investigations of contaminant electrons undertaken as part of this study show that contaminant electrons reaching the treatment field originate in the source container (ending at $Z=0$ in the BEAMnrc simulation geometry), the primary collimator (ending at $Z=7.7 \mathrm{~cm}$ ) and the field-defining aperture (ending at $Z=27.4 \mathrm{~cm}$ ). Thus, to include all potential contaminant electrons, the electron splitting plane (where fat electrons are split nsplit times) is placed at the bottom of the fielddefining aperture (modeled using a PYRAMIDS geometry in BEAMnrc and beampp) with the Russian Roulette plane (below which Russian Roulette is not played with thin electrons) defined at $Z=27 \mathrm{~cm}$. Since there is no radial symmetry at the bottom of the aperture, the option to redistribute the split electrons evenly about their radial position at the electron splitting plane is not used. For more information about electron splitting with directional bremsstrahlung splitting, see the paper by Kawrakow et al[11].

Fluence calculations described below are performed with and without electron splitting, while the dose and chamber calculations all include contaminant electrons.

\subsubsection{Fluence calculations}

Fluence at the bottom of the accelerator is scored using a custom EGSnrc user code, fluxyznrc, which scores planar fluence divided by the $\mathrm{Z}$ direction cosine (thus, an estimate of the actual fluence) in rectangular scoring zones. The simulation time for the fluxyznrc geometry is negligible compared to that for the Co-60 shared library source, so fluence efficiencies determined using fluxyznrc are the same as those of a stand-alone Co-60 simulation. Note that the grid fluence scoring option in BEAMnrc[8] could also have been used to determine fluence and fluence efficiency. 


\subsubsection{Dose calculations}

Doses are calculated in a water phantom with $\mathrm{X}, \mathrm{Y}$ and $\mathrm{Z}$ dimensions of $20.5 \mathrm{~cm}, 20.5 \mathrm{~cm}$ and $30 \mathrm{~cm}$, respectively, using the EGSnrc user code, DOSXYZnrc[13]. Dose scoring voxels are $0.5 \mathrm{~cm} \times 0.5 \mathrm{~cm} \times$ $0.5 \mathrm{~cm}$. ECUT and PCUT in the phantom are $0.7 \mathrm{MeV}$ and $0.01 \mathrm{MeV}$, respectively. In order to increase dose calculation efficiency in the phantom, photons are split 32 times (with weight reduced by $1 / 32$ ) with interaction sites distributed evenly along the original photon path. For more information about photon splitting in DOSXYZnrc, see the paper by Kawrakow and Walters[16]. In order to prevent higher-weight electrons from compromising statistics in the dose phantoms, contaminant electrons in the Co- 60 beam are split 32 times upon entering the phantom. In addition, the "HOWFARLESS" option for increasing the efficiency of dose calculations in a homogeneous phantom by speeding up charged particle transport is also used. For more information on "HOWFARLESS", see the paper by Walters and Kawrakow[17].

In these dose calculations, the air gap between the bottom of the field-defining aperture and the source-to-surface distance $(\mathrm{ssd}=100 \mathrm{~cm}$ ) is included in the DOSXYZnrc simulation instead of the Co60 simulation as this has been found[16] to be slightly more efficient when photon splitting is used.

\subsubsection{Chamber calculations}

The beam quality correction factor, $\mathrm{k}_{Q}$, for an ion chamber is given by[1]:

$$
k_{Q}=\frac{\left[D_{w} / D_{c}\right]^{Q}}{\left[D_{w} / D_{c}\right]^{60} C_{o}}
$$

where $\mathrm{Q}$ denotes the beam quality, $\mathrm{D}_{c}$ is the dose to the chamber, and $\mathrm{D}_{w}$ is the dose to a small volume of water at the same point of measurement. The Co-60 dependent ratio, $\left[D_{w} / D_{c}\right]^{60} \mathrm{Co}$, is measured or calculated at a reference depth of $5 \mathrm{~cm}$.

In this study, $\left[D_{w} / D_{c}\right]^{60} \mathrm{Co}$ is calculated for a simulated Farmer NE-2571 chamber. The NE-2571 is a thimble chamber commonly used for clinical linear accelerator calibrations. The geometry of the simulated NE-2571 chamber is based on that used by La Russa et al[18] and Muir and Rogers[19] and uses radiographic measurements of the chamber made at the National Research Council Canada. Simulations are performed using the egs_chamber user code, which uses the egspp C++ class library for EGSnrc[15] and which has been optimized for efficient calculations of in-phantom ion chamber dose by the introduction of photon cross-section enhancement in the chamber cavity volume and other variance reduction techniques[20]. The electron and photon transport cutoff energies, ECUT and PCUT, used in these simulations are $0.521 \mathrm{MeV}$ and $0.01 \mathrm{MeV}$, respectively.

The ratio, $\left[D_{w} / D_{c}\right]^{60} \mathrm{Co}$, for a $10 \times 10 \mathrm{~cm}^{2}$ field $(\mathrm{ssd}=100 \mathrm{~cm})$ calculated using a full, optimized BEAMnrc simulation of the Co-60 beam as a shared library source is compared with the same ratio calculated using a simple photon point source with an energy spectrum derived from phase space data collected at the bottom of the optimized BEAMnrc simulation. Phase space data are collected for $55 \times 10^{6}$ photons, representing $10 \times 10^{6}$ primary histories, and is sufficient to obtain $0.1 \%$ uncertainty on spectral 
peaks with an energy resolution of $0.01 \mathrm{MeV}$. In the case of the full simulation source, contaminant electrons are included in the chamber calculation.

\subsection{Efficiency}

The efficiency, $\epsilon$, of a simulation is given by:

$$
\epsilon=\frac{1}{T \sigma^{2}}
$$

where $\sigma$ is uncertainty, usually estimated as the standard deviation, on the quantity of interest (i.e. dose or fluence), and $T$ is the CPU time required to obtain the value of $\sigma$.

In this study, efficiency calculations are performed on $1.8 \mathrm{GHz}$ Opteron $244 \mathrm{CPU}$ 's. Every calculation is broken up into 15 separate jobs to allow uncertainties on the efficiencies to be estimated.

\subsection{Results}

\subsubsection{Fluence accuracy}

Figure 5.2 shows the fractional difference between fluence calculated with and without DSB for photons and contaminant electrons. Particle fluence scoring zones in the fluxyznrc code are $2 \mathrm{~cm} \times 10 \mathrm{~cm}$ (i.e. the entire Y-dimension of the field is included). The large scoring zones are used so that high precision can be obtained in a reasonable amount of time. Without DSB, photon fluences are calculated to within $0.05 \%$ uncertainty and electron fluences are calculated to within $1.5 \%$ uncertainty (with reasonable simulation times becoming the limiting factor), while with DSB, photon fluence is calculated to within $0.03 \%$ uncertainty and electron fluence to within $0.8 \%$ uncertainty.

Figure 5.2 shows agreement between photon fluence estimated with DSB and without DSB to within $2 \sigma$ across the treatment field. Given this level of agreement, there is a 5\% likelihood that the apparent systematic overestimation of photon fluence with DSB may be due to statistics in the calculation without DSB. Given the high precision of these calculations, however, any systematic difference due to DSB will be insignificant in practical applications. Increasing the precision of the calculation without DSB to confirm whether or not the difference is statistically significant is impractical given the length of time already required to achieve $0.05 \%$ uncertainty. Figure 5.2 shows that, within the precision available from the simulation with no DSB, no significant change in electron fluence occurs when DSB is used. Differences similar to those in photon fluence might occur if electron fluence was calculated down to similarly high precision. In addition, Figure 5.2 shows that DSB calculations using BEAMnrc and beampp are in good agreement. 


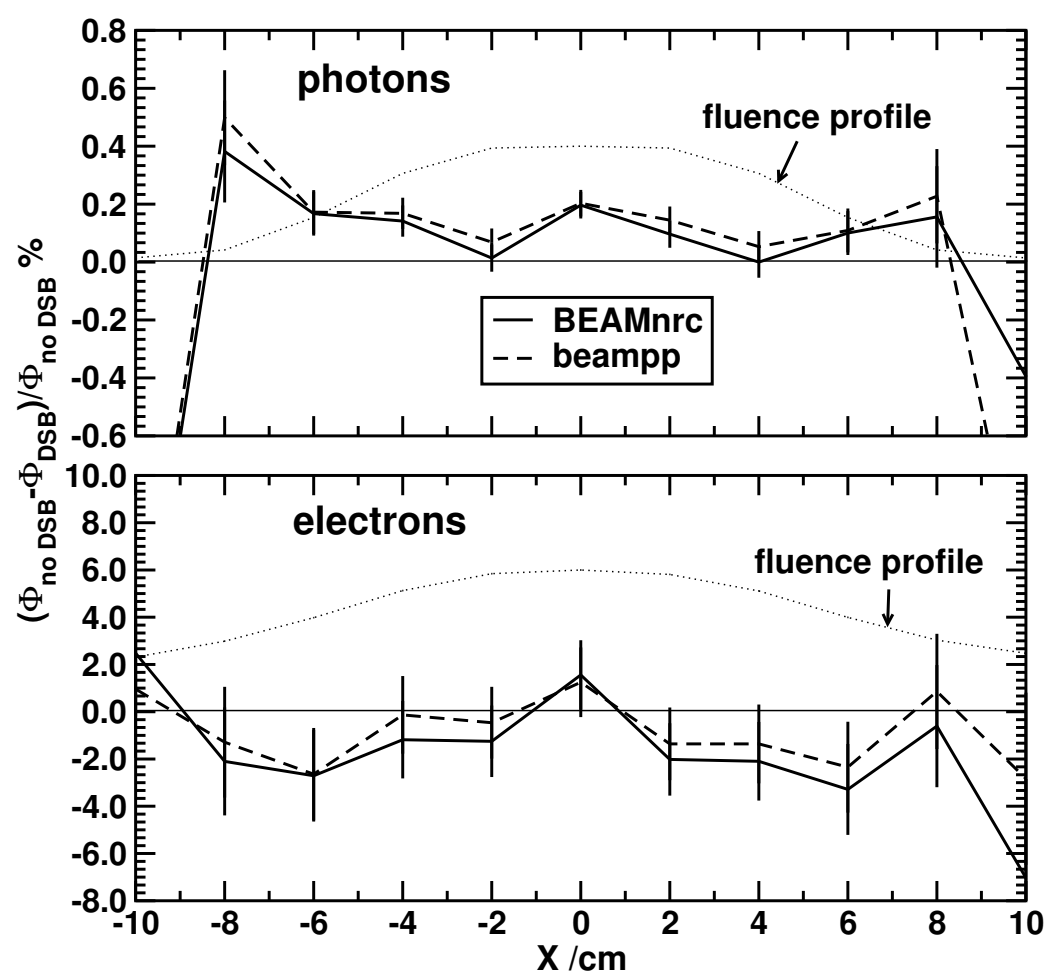

Figure 5.2: $\mathrm{X}$-direction profile of the fractional difference between particle fluence calculated without source biasing (DSB) and with DSB in the $10 \times 10 \mathrm{~cm}^{2}$ field $(\mathrm{ssd}=100 \mathrm{~cm})$ of a simulated Co-60 beam. Results are shown for photons and electrons. The DSB simulations are performed with nsplit $=2000$ and electron splitting below the field-defining aperture. Calculations with DSB are done using both BEAMnrc (differences shown as solid lines) and beampp (differences shown as dashed lines) simulation sources, while the reference calculation without DSB is performed using a BEAMnrc simulation source. Fluence scoring zones are $2 \mathrm{~cm} \times 10 \mathrm{~cm}$. Normalized photon and electron particle fluence profiles are also shown.

\subsubsection{Fluence calculation efficiency}

Figure 5.3 shows the total calculation efficiency of photon and electron fluence inside the $10 \times 10 \mathrm{~cm}^{2}$ field as a function of nsplit. Efficiency is expressed relative to that without DSB.

Figure 5.3 indicates that the maximum photon fluence efficiency occurs at nsplit $\approx 20,000$ for Co- 60 simulations using BEAMnrc and at nsplit $\approx 30,000$ for simulations using beampp. The efficiency shows little variation around the maximum, so the exact choice of nsplit is not critical. Also note that the generation of contaminant electrons does not significantly decrease the photon fluence efficiency. This implies that tracking the additional electrons through the airgap below the field-defining aperture does not significantly increase the simulation time.

Maximum efficiency of the contaminant electron fluence calculation occurs at lower values of nsplit, 


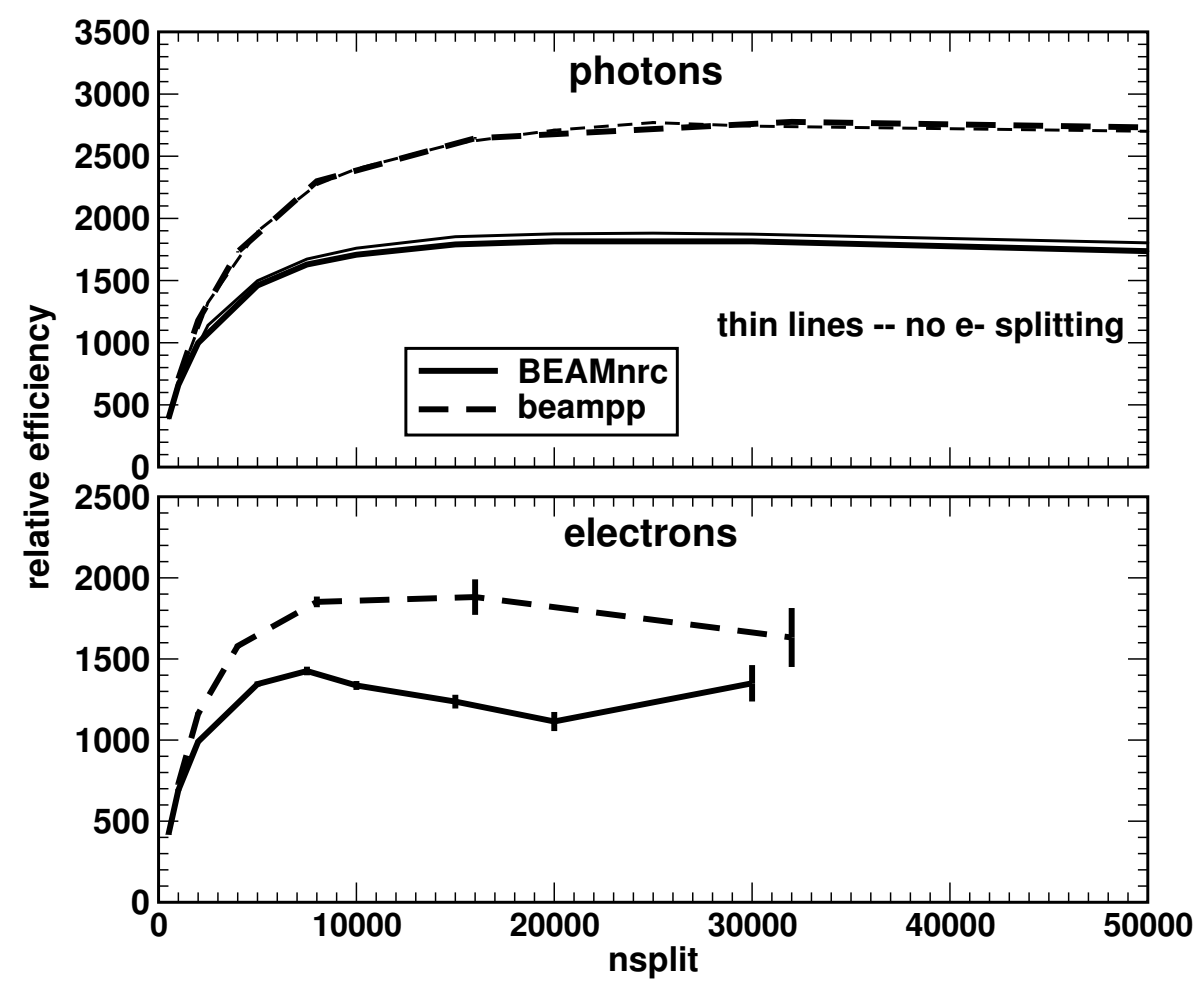

Figure 5.3: Total calculation efficiency of photon and electron particle fluence inside the $10 \times 10 \mathrm{~cm}^{2}$ field ( $\mathrm{ssd}=100 \mathrm{~cm}$ ) of Co-60 beams simulated with DSB as a function of the splitting number, nsplit. Efficiencies are relative to the BEAMnrc simulation without DSB. Particle fluence is scored in $1 \times 1 \mathrm{~cm}^{2}$ zones and total efficiency is calculated by summing the squares of the uncertainties in each zone contained within the field and using this as $\sigma^{2}$ in Equation (5.10). Simulations with DSB are performed with BEAMnrc (solid lines) and beampp (dashed lines). Photon fluence efficiency is also shown for simulations in which contaminant electrons are not generated (thin lines).

with maxima at nsplit $\approx 7,500$ for the BEAMnrc simulation and nsplit $\approx 15,000$ for the beampp simulation. With increasing nsplit, the uncertainty in electron fluence efficiency increases. The arrival of a contaminant electron in the field is a low-probability event, and, thus, the increased variability in electron fluence with increasing nsplit is expected. Since electron fluence is such a small fraction $(\sim 0.1 \%)$ of the total fluence, the optimum setting of nsplit is chosen to be that which maximizes photon fluence efficiency.

Figure 5.4 shows photon and electron particle fluence efficiency in $1 \times 1 \mathrm{~cm}^{2}$ scoring zones along the $\mathrm{X}$-axis of the treatment field. The values of nsplit for the simulations shown here are chosen to be at or near maximum photon fluence efficiency in Figure 5.3.

Figure 5.4 shows that the gain in photon fluence efficiency due to DSB is almost constant across the $10 \times 10 \mathrm{~cm}^{2}$ field. The electron fluence efficiency gains have high uncertainty due to the large values of 


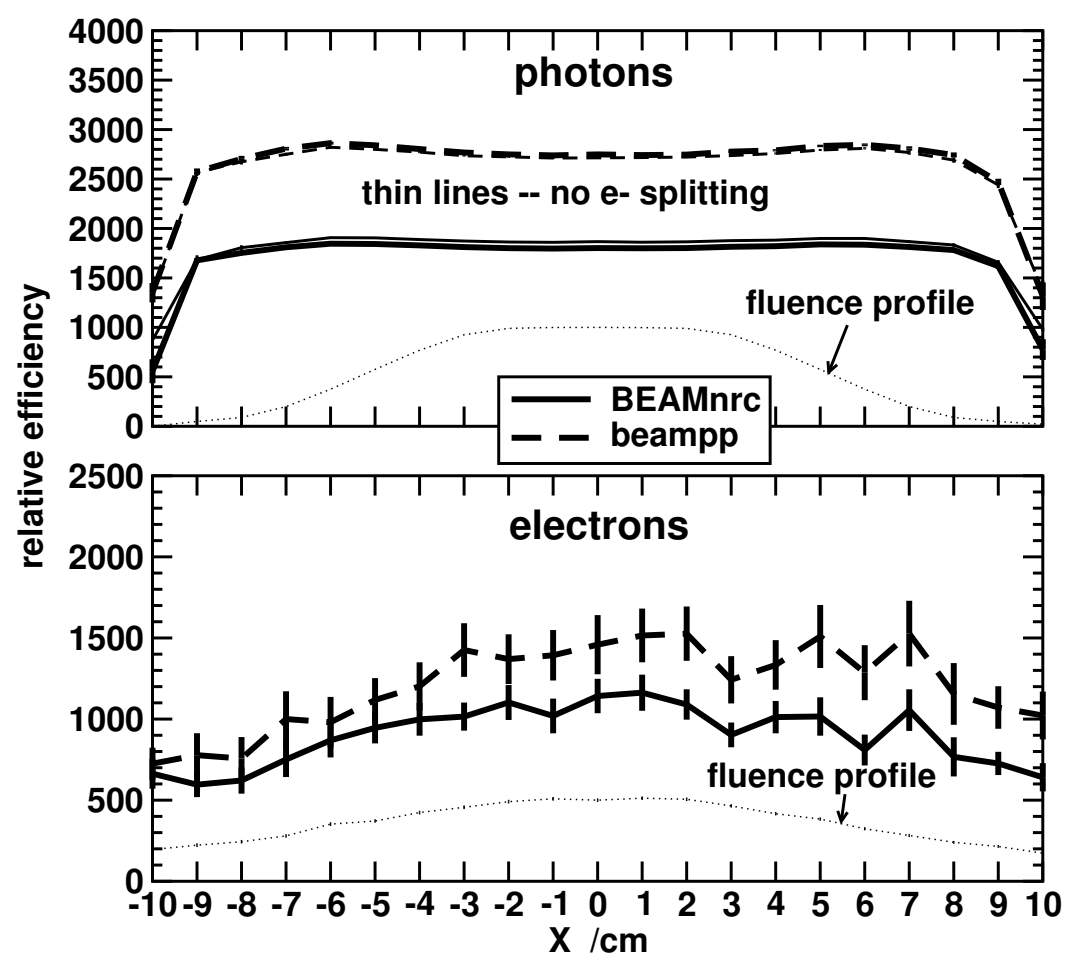

Figure 5.4: Calculation efficiency of photon and electron particle fluence in $1 \times 1 \mathrm{~cm}^{2}$ scoring zones along the field X-axis in $10 \times 10 \mathrm{~cm}^{2} \mathrm{Co}-60$ beams $(\mathrm{ssd}=100 \mathrm{~cm}$ ) simulated with DSB. The splitting field radius, $\mathrm{fs}$, is $10 \mathrm{~cm}$. Efficiencies are relative to the BEAMnrc simulation with no DSB. The fluence profiles are also shown for reference. The splitting numbers (nsplit) used are 20,000 for simulations using BEAMnrc (with and without electron splitting to generate contaminant electrons), 32,000 for the beampp simulation with electron splitting, and 30,000 for the beampp simulation without electron splitting. These values of nsplit result in maximum, or near-maximum, photon fluence efficiency.

nsplit necessary to maximize photon fluence efficiency (see above for a more detailed explanation).

Figure 5.5 shows the variation in total fluence efficiency for photons and electrons in the field as a function of $\Delta$, the minimum linear distance (as projected to the $s s d$ of $100 \mathrm{~cm}$ ) between photons when they are split and redistributed about their radial position at the DSB splitting plane.

From Figure 5.5 it is evident that fluence efficiency does not vary greatly over a large $\Delta$ range $(1 \mathrm{~cm}-$ $2.5 \mathrm{~cm}$ ). The value of $\Delta$ used for all other simulations in this study is $1.5 \mathrm{~cm}$, which gives near-maximum efficiency gains for photon fluence.

Overall, the scheme of splitting and redistributing photons upon leaving the radially symmetric portion of the treatment head increases the maximum total fluence efficiency (obtained with nsplit $=20,000$ and $\Delta=1.5 \mathrm{~cm}$ ) by $\sim 20 \%$ for the photon fluence calculation and $\sim 15 \%$ for the contaminant electron fluence calculation. 


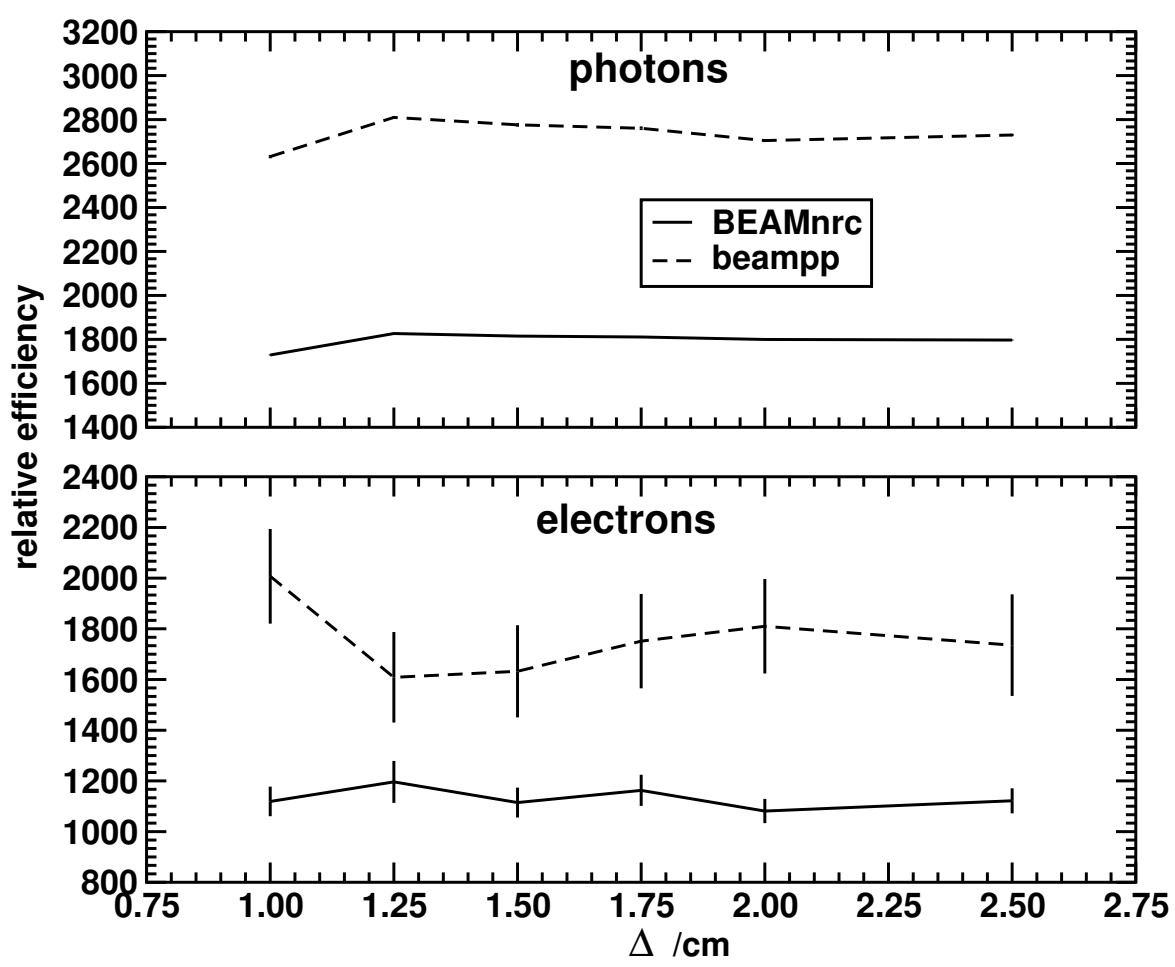

Figure 5.5: Calculation efficiency of total photon and electron fluence inside the $10 \times 10 \mathrm{~cm}^{2}$ field $(\mathrm{ssd}=100 \mathrm{~cm})$ of the Co-60 beam simulations as a function of the DSB parameter, $\Delta$, the minimum linear distance (projected to ssd) between photons when they are split and redistributed about their radial position at the splitting plane (the top of the primary collimator). The splitting field radius, $f \mathbf{s}$, is $10 \mathrm{~cm}$. Efficiencies are relative to the BEAMnrc calculation without DSB. For BEAMnrc simulations nsplit $=20,000$ and for beampp simulations nsplit $=32,000$, which result in maximum photon fluence efficiency.

\subsubsection{Dose calculation efficiency}

Figure 5.6 shows the total calculation efficiency of doses $>0.5 \times \mathrm{D}_{\max }$ as a function of nsplit for the Co-60 beam incident on a water phantom. Doses are calculated to within $0.5 \%$ uncertainty in simulations using DSB and to within $1.5 \%$ uncertainty without DSB. The variance reduction techniques used in DOSXYZnrc ("HOWFARLESS" and photon splitting) increase the efficiency of the reference (BEAMnrc with no DSB) calculation by a factor of approximately 30 . Note the dotted line showing the relative efficiency of the calculation without DSB using the time-saving strategies of Mora et al[10] (see Section III.A above): high values of ECUT in the source container and range rejection at all energies.

Similar to fluence efficiency as a function of nsplit (see Figure 5.3), the dose calculation efficiency with DSB is maximized at splitting numbers in the range 20,000-40,000, with the optimum value of 


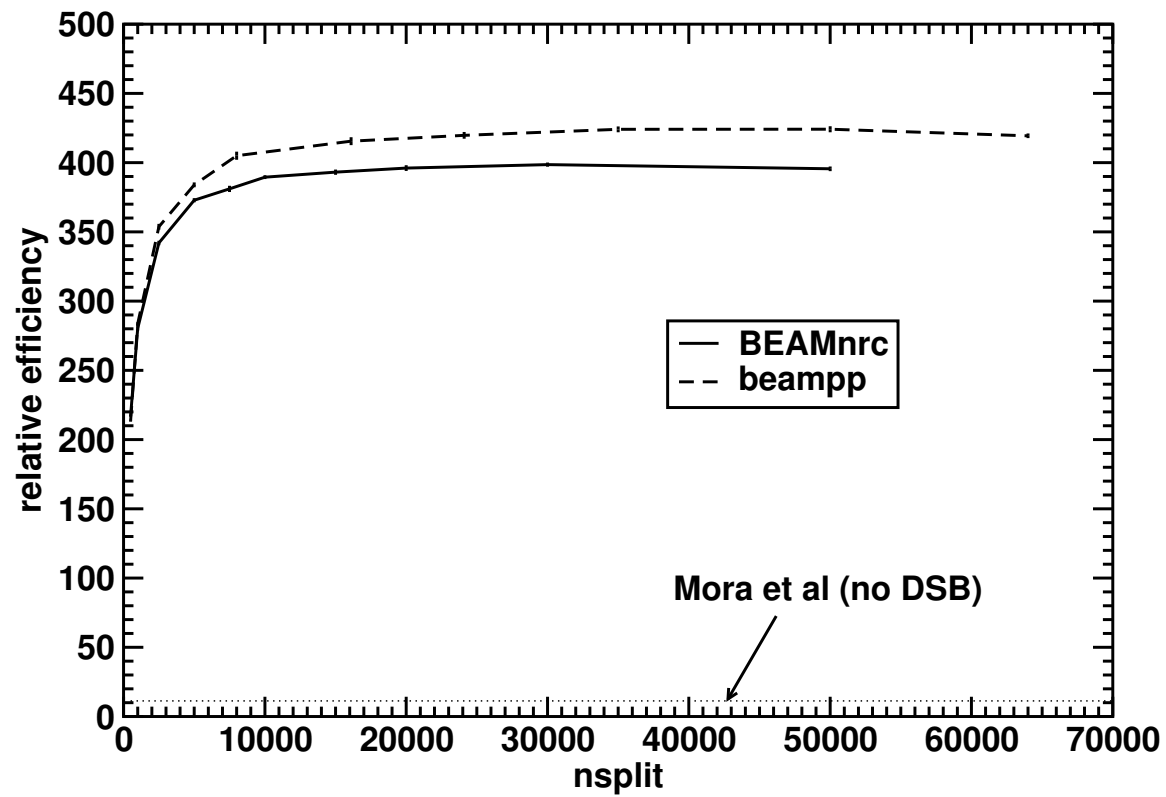

Figure 5.6: Total calculation efficiency of doses $>0.5 \times \mathrm{D}_{\max }$ as a function of the splitting number, nsplit, for the $10 \times 10 \mathrm{~cm}^{2} \mathrm{Co}-60$ beam incident on a water phantom. Efficiencies are relative to the efficiency using BEAMnrc without DSB. The water phantom is modeled using DOSXYZnrc and has overall $\mathrm{X}, \mathrm{Y}, \mathrm{Z}$ dimensions $20.5 \mathrm{~cm} \times 20.5 \mathrm{~cm} \times 30 \mathrm{~cm}$ divided into $0.5 \mathrm{~cm} \times 0.5 \mathrm{~cm} \times 0.5 \mathrm{~cm}$ voxels. Results are shown for simulations using DSB with BEAMnrc (solid line) and with beampp (dashed line). Doses are calculated to within $0.5 \%$ uncertainty in simulations using DSB and to within $1.5 \%$ uncertainty without DSB. The dotted line shows the efficiency of the calculation without DSB using the strategy of varying ECUT and performing charged particle range rejection at all energies employed by Mora et al[10]. All calculations use $32 \times$ photon splitting and the "HOWFARLESS" option for speeding up charged particle transport in the DOSXYZnrc phantom. Doses include contributions from contaminant electrons. 
nsplit for BEAMnrc simulations being lower than that for beampp simulations. Also, the efficiency does not vary significantly over a large range of nsplit values around the maximum, so the exact setting of nsplit is not critical. Note that the maximum dose efficiency of DSB simulations using beampp is only $~ 5 \%$ higher than the maximum efficiency of DSB simulations using BEAMnrc. This is in contrast to fluence efficiency, where the maximum efficiency of photon fluence calculated with beampp is $\sim 55 \%$ higher than that calculated with BEAMnrc, and shows that, with highly-efficient beam simulations, the time required for the DOSXYZnrc phantom calculation becomes the dominant factor in the overall efficiency.

The gain in dose calculation efficiency achieved with DSB is approximately 40 times greater than that obtained using the time-saving techniques of Mora et al[10]. Thus, the calculation mentioned in the Introduction that requires over $1,500 \mathrm{~h}$ on a $1.8 \mathrm{GHz}$ Opteron $244 \mathrm{CPU}$ to obtain $<0.1 \%$ dose uncertainty now requires only $\sim 37.5 \mathrm{~h}$. Moreover, the use of Mora et al's parameters has been found to result in inaccuracies in fluence/primary history (see Section III.A) which will result in dose inaccuracies.

\subsection{Chamber calculations}

Based on the fluence efficiency results in Section V.B above, the BEAMnrc simulation of the Co- 60 beam used to calculate $\left[D_{w} / D_{c}\right]^{60} \mathrm{Co}$ is optimized using a DSB splitting number, nsplit, of 20,000 and a minimum linear distance between redistributed split photons, $\Delta$, of $1.5 \mathrm{~cm}$.

$\mathrm{D}_{c}$ and $\mathrm{D}_{w}$ are calculated to within $0.1 \%$ uncertainty, requiring total cpu times of $6.9 \mathrm{~h}$ and $2.3 \mathrm{~h}$, respectively, for calculations using the full Co-60 simulation source, and $4.9 \mathrm{~h}$ and $1.3 \mathrm{~h}$, respectively, for calculations using the photon point source.

The value of $\left[\frac{D_{w}}{D_{c}}\right]^{60} \mathrm{Co}$ calculated at a depth of $5 \mathrm{~cm}$ for a $10 \mathrm{~cm} \times 10 \mathrm{~cm}$ field $(\mathrm{ssd}=100 \mathrm{~cm})$ using the full Co-60 beam simulation source is $1.109 \pm 0.002$. The value of $\left[\frac{D_{w}}{D_{c}}\right]^{60} \mathrm{Co}$ calculated using the photon point source is $1.108 \pm 0.002$. Agreement at this level has also been observed by Muir and Rogers[19] using a phase space source derived from a BEAMnrc Co- 60 treatment head simulation and confirms the negligible effect of contaminant electrons at $5 \mathrm{~cm}$ depth and the unimportance of the geometric details of the Co-60 treatment head for these calibrations.

\subsection{Conclusion}

Using the technique of splitting primary photons emitted by the source and sampling only those split photons directed into a user-defined (encompassing the treatment field), directional source biasing (DSB) greatly increases the efficiency of Co-60 treatment head simulations $\left(10 \times 10 \mathrm{~cm}^{2}\right.$ field). Total efficiency of in-field photon fluence increases by a factor of approximately 1,800 when the treatment head is simulated using BEAMnrc and approximately 2,800 when the treatment head is simulated with beampp, an accelerator simulation code using the egspp $\mathrm{C}++$ class library. Total efficiency of contaminent electron fluence increases by approximately 1,200 with BEAMnrc and approximately 1,600 with beampp. Total 
dose calculation efficiency increases by a factor of approximately 400 . Even when cpu time is saved in the calculation without DSB by setting high values of ECUT in the source capsule and using charged particle range rejection at all energies, the calculation with DSB is approximately 40 times more efficient.

The DSB algorithm also saves computation time by taking advantage of the radial symmetry at the top of the Co-60 treatment head to reduce the number of photons tracked there and by playing Russian Roulette with secondary electrons, regenerating the latter if electron contamination is to be included.

Together with variance reduction techniques already in place in DOSXYZnrc (photon splitting, "HOWFARLESS"), DSB allows calculations that were previously prohibitively long to be done in a reasonable amount of time. The photon splitting numbers (nsplit) at which DSB reaches maximum efficiency are in the range 20,000-40,000. The optimum splitting number is likely to vary depending on field size and scoring voxel size, however efficiency does not vary greatly about the maximum value, and a setting of nsplit=20,000 should achieve near-maximum efficiency.

Use of DSB opens up the possibility of regularly performing Monte Carlo Co-60 treatment head simulations which can be used to commission Co- 60 beams for use in IMRT and IGRT. Also, since Co60 is used as the reference in determining beam quality factors and correction factors, DSB facilitates accurate calculations of these in a reasonable time. This is illustrated above by the example calculation of $\left[D_{w} / D_{c}\right]^{60} \mathrm{Co}$ in a realistic ion chamber using a full BEAMnrc simulation of a Co-60 treatment head as a source.

Finally, DSB is not limited to Co-60 sources but will increase the efficiency of other isotropically radiating sources, such as Cs-137, making simulation of these sources more practical. The DSB option has been implemented in the current release of BEAMnrc, and documentation will be included in future releases of the BEAMnrc Users Manual.

\section{Acknowledgements}

The author wishes to acknowledge Iwan Kawrakow, currently at ViewRay, who came up with the initial concept for DSB and coded extensive portions of the algorithm during his tenure at the National Research Council Canada (NRCC). The author also acknowledges Bryan Muir at the NRCC for providing details of the NE2571 chamber and his MC calculations of $\mathrm{k}_{Q}$. Malcolm McEwen and Frédéric Tessier at the NRCC provided valuable comments on early drafts of the manuscript. Ernesto Mainegra-Hing of the NRCC is acknowledged for encouraging the publication of this work. Finally, the author wishes to thank David Rogers at Carleton University for many useful comments on later versions of the manuscript.

\subsection{Bibliography}

[1] P. R. Almond, P. J. Biggs, B. M. Coursey, W. F. Hanson, M. S. Huq, R. Nath, and D. W. O. Rogers. AAPM's TG-51 Protocol for Clinical Reference Dosimetry of High-Energy Photon and Electron Beams. Med. Phys., 26:1847 - 1870, 1999. 
[2] M. McEwen, L. DeWerd, G. Ibbott, D. Followill, D. W. O. Rogers, S. Seltzer, and J. Seuntjens. Addendum to AAPM's TG-51 protocol for clinical reference dosimetry of high-energy photon beams. Med. Phys., 41:041501-1 - 041501-20, 2014.

[3] C. P. Joshi, J. Darko, P. B. Vidyasagar, and L. J. Schreiner. Investigation of an efficient source design for Cobalt-60-based tomotherapy using EGSnrc Monte Carlo Simulation. Phys Med Biol, 53:575$592,2008$.

[4] C. Fox, H. E. Romeijn, B. Lynch, C. Men, D. M. Aleman, and J. F. Dempsey. Comparative analysis of ${ }^{60}$ Co intensity-modulated radiation therapy. Phys. Med. Biol., 53:3175-3188, 2008.

[5] L. J. Schreiner, C. P. Joshi, J. Darko, A. Kerr, G. Salomons, and S. Dhanesar. The role of Cobalt-60 in modern radiation therapy: Dose delivery and image guidance. J Med Phys, 34:133-136, 2009.

[6] J. Dempsey, B. Dionne, J. Fitzsimmons, A. Haghigat, J. Li, D. Low, S. Mutic, J. Palta, H. Romeijn, and G. Sjoden. A Real-Time MRI Guided External Beam Radiotherapy Delivery System. Med. Phys. (abstract), 33:2254, 2006.

[7] T. Kron, D. Eyles, L. J. Schreiner, and J. Battista. Magnetic resonance imaging for adaptive cobalt tomotherapy: A proposal. J. Med. Phys., 31:242-254, 2006.

[8] D. W. O. Rogers, B. Walters, and I. Kawrakow. BEAMnrc Users Manual. Technical report, 2005.

[9] I. Kawrakow and D. W. O. Rogers. The EGSnrc Code System: Monte Carlo simulation of electron and photon transport. Technical Report PIRS-701 (4th printing), National Research Council of Canada, Ottawa, Canada, 2003.

[10] G. Mora, A. Maio, and D. W. O. Rogers. Monte Carlo simulation of a typical ${ }^{60}$ Co therapy source. Med. Phys., 26:2494 - 2502, 1999.

[11] Iwan Kawrakow, D. W. O Rogers, and B.R.B. Walters. Large efficiency improvements in BEAMnrc using directional bremsstrahlung splitting. Med. Phys., 31:2883 - 2898, 2004.

[12] A. F. Bielajew, D. W. O. Rogers, and A. E. Nahum. Monte Carlo simulation of ion chamber response to ${ }^{60} \mathrm{Co}-$ Resolution of anomalies associated with interfaces. Phys. Med. Biol., 30:419 - 428, 1985.

[13] B. R. B. Walters, I. Kawrakow, and D. W. O. Rogers. DOSXYZnrc Users Manual. NRC Report PIRS 794 (rev B), 2005.

[14] D. W. O. Rogers, I. Kawrakow, J. P. Seuntjens, B. R. B. Walters, and E. Mainegra-Hing. NRC User Codes for EGSnrc. Technical Report PIRS-702(RevC), National Research Council of Canada, Ottawa, Canada, 2010.

[15] I. Kawrakow. egspp: the EGSnrc C++ class library. Technical Report PIRS-899, National Research Council of Canada, Ottawa, Canada, 2005. 
[16] I. Kawrakow and B. R. B. Walters. Efficient photon beam dose calculations using DOSXYZnrc with BEAMnrc. Med. Phys., 33:3046 - 3056, 2006.

[17] B. R. B. Walters and I. Kawrakow. A "HOWFARLESS" option to increase efficiency of homogeneous phantom calculations with DOSXYZnrc. Med. Phys., 34:3794 - 3807, 2007.

[18] D. J. La Russa, M.R. McEwen, and D. W. O. Rogers. An experimental and computational investigation of the standard temperature-pressure correction factor for ion chambers in kilovoltage $\mathrm{x}$ rays. Med. Phys., 34:4690-4699, 2007. http://link.aip.org/link/?MPH/34/4690/1.

[19] B. R. Muir and D. W. O. Rogers. Monte Carlo calculations of $\mathrm{kQ}$, the beam quality conversion factor. Med. Phys., 37:5939-5950, 2010.

[20] Jörg Wulff, Klemens Zink, and Iwan Kawrakow. Efficiency improvements for ion chamber calculations in high energy photon beams. Med. Phys., 35:1328-1336, 2008. 


\section{VI}

Skeletal dosimetry in cone beam computed tomography

B. R. B. Walters, G. X. Ding, R. Kramer, I. Kawrakow Medical Physics July 2009: Vol. 36: No. 7: 2915-2922 


\begin{abstract}
Cone beam computed tomography $(\mathrm{CBCT})$ is a relatively new patient imaging technique that has proved invaluable for treatment target verification and patient positioning during image-guided radiotherapy (IGRT). It has been shown that CBCT results in additional dose to bone that may amount to $10 \%$ of the prescribed dose. In this study, voxelized human phantoms, FAX06 (adult female) and MAX06 (adult male), are used together with phase-space data collected from a realistic model of a CBCT imager to calculate dose in the red bone marrow (RBM) and bone surface cells (BSC), the two organs at risk within the bone spongiosa, during simulated head and neck, chest and pelvis CBCT scans. The FAX06/MAX06 phantoms model spongiosa based on micro-CT images, filling the relevant phantom voxels, which are $0.12 \times 0.12 \times 0.12 \mathrm{~cm}^{3}$, with $17 \times 17 \times 17 \mu \mathrm{m}^{3}$ micro voxels to form a micro-matrix of trabecular bone and bone marrow. FAX06/MAX06 have already been implemented in an EGSnrc-based Monte Carlo code to simulate radiation transport in the phantoms, however this study required significant modifications of the code to allow use of phase-space data from a simulated $\mathrm{CBCT}$ imager as a source and to allow scoring of total dose, RBM dose and BSC dose on a voxel-by-voxel basis. In simulated CBCT scans, the BSC dose is significantly greater than the dose to other organs at risk. For example, in a simulated head and neck scan, average BSC dose is $25 \%$ higher than the average dose to eye lens ( $8.3 \mathrm{cGy}$ ), and $80 \%$ greater than the average dose to brain (5.7 cGy). Average dose to RBM, on the other hand, is typically only $\sim 50 \%$ of the average BSC dose and less than the dose to other organs at risk ( $54 \%$ of the dose to eye lens and $76 \%$ of dose to brain in a head and neck scan). Thus, elevated dose in bone due to CBCT results in elevated BSC dose. This is potentially of concern when using $\mathrm{CBCT}$ in conjunction with radiotherapy treatment.
\end{abstract}




\subsection{Introduction}

With the advance of imaging technology the Image-guided radiation therapy (IGRT) has become the new paradigm in radiotherapy. A promising new technique in image-guided radiotherapy (IGRT) is cone-beam computed tomography $(\mathrm{CBCT})[1-8]$. Using kilovoltage $(\mathrm{kV})$ photon beams, CBCT can provide accurate 3-D volumetric knowledge about patient anatomy for every treatment fraction and is suitable for adaptive corrections of treatment positioning variations $[1,2,9,10]$. Recent studies demonstrate that imaging guidance procedures in radiotherapy may add significant additional doses to the normal tissues[11-14]. Therefore information regarding additional dose due to CBCT is important to clinicians in order to make informed decisions regarding its frequency of use.

Unlike in photon beams in the megavoltage energy range there are large variations in mass-energy absorption coefficients between bone and soft tissues due to the photoelectric effect in the kilovoltage range[15-17]. Recent studies[14, 18] have demonstrated that the patient bony anatomy receives up to a factor of 2-4 higher dose than soft tissues in a typical $\mathrm{kV} \mathrm{CBCT} \mathrm{scan.} \mathrm{During} \mathrm{a} \mathrm{treatment} \mathrm{procedure,} \mathrm{this}$ translates into an additional dose to bone that may amount to $10 \%$ of the prescribed dose. The organs at risk within the skeleton, as identified by the ICRP[19], are the haematopoietic stem cells of the bone marrow, called "red bone marrow" (RBM), and the osteogenic cells on the endosteal surfaces, called "bone surface cells" (BSC). The RBM consists mainly of myeloid tissue which gives rise to red blood cells, platelets and most white blood cells. The BSC layer is currently considered to have a thickness of $10 \mu \mathrm{m}[19]$. The bone marrow, together with other skeletal soft tissue and its supporting structure of trabecular bone, comprise what is known as the spongiosa[20]. Spongiosa is found throughout the skeleton mainly in the flat bones, such as the hip bone, breast bone, skull, ribs, vertebrae, shoulder blades, and at the proximal ends of the femur and humerus.

This study presents Monte Carlo calculations of dose in realistic human female/male phantoms (FAX06/MAX06) during simulated head and neck, chest and pelvic CBCT scans. The FAX06/MAX06 phantoms allow dose to spongiosa to be decomposed into dose to RBM and dose to BSC in an attempt to determine if the elevated bone dose due to CBCT translates into elevated dose in these sensitive organs.

Section 6.2 introduces the FAX06/MAX06 phantoms developed by Kramer et al[21] including a detailed description of how the spongiosa is modeled. Modifications to the EGSnrc-based[22, 23] user codes which model transport in FAX06/MAX06 to allow the use of phase-space sources and dose scoring on a voxel-by-voxel basis are described in Section 6.2.1. Section 6.3 briefly describes the phase-space source provided by Ding et al[14] from their BEAMnrc[24] Monte Carlo simulation of a Varian OBI CBCT system, and Section 6.4 describes the procedure developed by Ding et al[14] to calibrate Monte Carlo-calculated dose. The simulation parameters used in the FAX06/MAX06 dose calculations for the 3 different scans are described in Section 6.5. Dose results, including cumulative dose volume histograms (DVH's) for various organs (including RBM and BSC) are shown in Section 6.6. Concluding remarks appear in Section 6.7. 


\subsection{The FAX06/MAX06 voxelized human phantoms}

The FAX06/MAX06 voxelized adult female/male phantoms were developed for radiation protection dosimetry and are described in detail by Kramer et al[21]. These phantoms have been reconstructed from segmented CT images of a female and male patient. Phantoms are divided into $0.12 \times 0.12 \times 0.12 \mathrm{~cm}^{3}$ voxels and have lateral width $56.88 \mathrm{~cm}$ (474 voxels), anterior-posterior depth $26.64 \mathrm{~cm}$ (222 voxels), and height $175.32 \mathrm{~cm}$ (1461 voxels) for the male and $163.08 \mathrm{~cm}$ (1359 voxels) for the female. Thus, MAX06 has a total of 153,738,108 voxels, and FAX06 has 143,004,852 voxels. Note that not all voxels are filled with tissue, as air outside any parts of the body that do not extend the full lateral width or anterior-posterior depth and air between non-contiguous parts of the body (e.g. between the legs) is also modeled. The X$\mathrm{Y}-\mathrm{Z}$ coordinate system of the phantom is a right-handed system defined such that the $\mathrm{X}$-axis is from the phantom's left to right in the lateral direction, the Y-axis is anterior-posterior, and the Z-axis goes from the head to the feet of the phantom. The origin is defined such that the phantom contains no negative coordinates (i.e. it is the top left front corner of the phantom). Organ volumes and masses and tissue composition in FAX06/MAX06 are based on up-to-date values recommended by the International Commission on Radiological Protection (ICRP)[25] and the International Commission on Radiation Units (ICRU)[26].

In a further refinement of the FAX06/MAX06 phantoms that is particularly useful for this study, Kramer et al[27] introduced precise modeling of spongiosa in the skeleton based on micro-CT images. Spongiosa is defined as trabecular bone plus its supported soft tissue, including the organs at risk: red bone marrow (RBM) and bone surface cells (BSC). For a more complete description of the spongiosa, see Section 6.1 above. FAX06/MAX06 models spongiosa by dividing spongiosa voxels into micro-matrices. A micro-matrix consists of micro voxels with resolution $17.65 \mu \mathrm{m}(68 \times 68 \times 68$ micro voxels $), 30 \mu \mathrm{m}$ $(40 \times 40 \times 40$ micro voxels $)$ or $60 \mu \mathrm{m}(20 \times 20 \times 20$ micro voxels). The structure of micro-matrices is based on micro-CT images of spongiosa. Since it is computationally impractical to fill each of the $\sim 2$ million spongiosa voxels in a FAX06/MAX06 phantom with a different micro-matrix, for the purposes of dose calculations Kramer et al adopted a repeating structure of 8 different micro-matrices. These micromatrices are applied to the relevant spongiosa voxels on the fly during dose calculations. In addition to modeling a contiguous spongiosa structure, micro-matrices must also match the trabecular bone volume fractions (TBVFs) at different sites in the skeleton. Five different TBVFs throughout the human skeleton have been identified by the ICRP[20]: 10\% (ribs), 12\% (spine), 15\% (long bones), 20\% (pelvis) and 55\% (skull/mandible). Each of these TBVFs requires its own set of 8 micro-matrices. Thus, FAX06/MAX06 has data for 40 micro-matrices in all.

Figure 6.1 shows a slice through the chest $($ at $\mathrm{Z}=40 \mathrm{~cm}$ ) of the MAX06 phantom. The anatomical structures are clearly visible in this cross-section, with the black portions indicating the location of the spongiosa in the spine, humerus and rib cage. The top image is one of the 8 micro-matrices (dimensions $0.12 \mathrm{~cm} \times 0.12 \mathrm{~cm} \times 0.12 \mathrm{~cm}$ ) used to model the spongiosa in the location indicated (the humerus, $\mathrm{TBVF}=15 \%$ ). During particle transport through the spongiosa, the 8 micro-matrices are applied on the fly in a systematic periodic way to create a $2 \times 2 \times 2$ micro-matrix cluster, the structure of which is 
preserved and repeated as required.

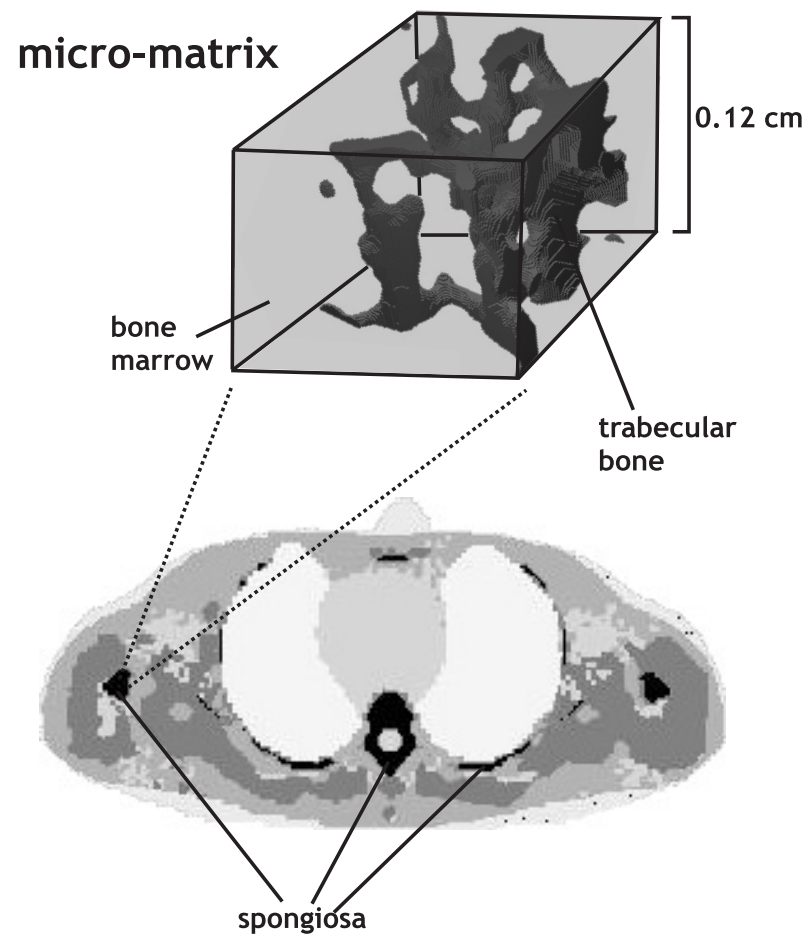

Figure 6.1: A slice (at $\mathrm{Z}=40 \mathrm{~cm}$ ) through the chest of the MAX06 phantom indicating the locations of spongiosa (black) in the spine, humerus and rib cage. The top image shows one of the 8 different micro-matrices $(0.12 \mathrm{~cm} \times 0.12 \mathrm{~cm} \times 0.12 \mathrm{~cm})$ used to model the spongiosa in the humerus (TBVF=15\%), indicating the trabecular bone and bone marrow portions. Each micro-matrix is comprised of $68 \times 68 \times 68$ micro voxels having dimensions 17.65 cubic $\mu \mathrm{m}$ and filled with either trabecular bone or bone marrow. Bone surface cells (BSC) are not modeled explicitly but for the purposes of dose deposition are considered to be a $10 \mu \mathrm{m}$ layer of bone marrow adjacent to trabecular bone.

In the first implementation of micro-matrices in the spongiosa of FAX06/MAX06 by Kramer et al[27], each micro voxel contained either trabecular bone, bone marrow (includes RBM) or BSC. As mentioned above, BSC is considered to be a $10 \mu \mathrm{m}$ layer covering the surfaces of trabecular bone, while the highest resolution micro voxels are 17.65 cubic $\mu \mathrm{m}$. Thus, actual BSC dose could only be arrived at by extrapolating results obtained using 60 cubic $\mu \mathrm{m}, 30$ cubic $\mu \mathrm{m}$ and 17.65 cubic $\mu \mathrm{m}$ micro voxels. A more recent approach[28], and the one employed in this study, uses only trabecular bone- and bone marrowfilled micro voxels and estimates BSC dose by considering the electron step length in bone marrow voxels. If dose is distributed evenly over the step (not strictly true since this does not account for the full curved path of the electron, but it has been found to be sufficiently accurate in such small micro voxels) then the BSC dose deposited during the step is the total dose deposited times the fraction of the step that is within 
$10 \mu \mathrm{m}$ of a voxel containing trabecular bone (note that this fraction can be zero). Using this method to estimate BSC dose, it may be thought that the resolution required in the micro-matrices can be relaxed somewhat (and dose computation time decreased) by using the 60 cubic $\mu \mathrm{m}$ micro voxels. However, Kramer et al[28] found significant differences between BSC doses calculated using the 60 cubic $\mu \mathrm{m}, 30$ cubic $\mu \mathrm{m}$ and 17.65 cubic $\mu \mathrm{m}$ micro voxels at low energies. Thus, in this study, the 17.65 cubic $\mu \mathrm{m}$ micro voxel resolution is used as it potentially provides the most accurate representation of spongiosa structure. In both the original and the more recent implementation of micro-matrices, dose to RBM is calculated by multiplying dose in bone marrow micro voxels by a cellularity factor representing the fraction of RBM in the marrow.

In the discussion that follows, micro voxels containing bone morrow are referred to as containing $\mathrm{RBM}$, since it is the RBM fraction of the marrow that is of interest.

\subsubsection{Modifications to FAX06/MAX06 user codes}

For the dose calculations described in references[27, 28], the FAX06/MAX06 phantoms were implemented in an EGSnrc-based user code. EGSnrc[22, 23] is a system of Monte Carlo codes for the accurate simulation of radiation transport. The existing FAX06/MAX06 user code allowed calculation of whole body and bone-specific doses to RBM, BSC and total doses to many soft tissue organs for a variety of radiation sources including exterior parallel beams and point sources of user-specified charge, energy and field size, and internal sources of user-specified charge and energy emitting from a user-specified organ.

This study required implementation of a phase-space source in the FAX06/MAX06 user code. The phase-space source makes use of the phase-space handling macros included as part of the EGSnrc code system[23] (and used to implement phase-space sources in many existing EGSnrc user codes) to read phase-space data output by BEAMnrc[24] simulations. Figure 6.2 shows the phase-space source incident in a simulated head and neck scan. The source allows the user to specify an isocentre anywhere within the FAX06/MAX06 phantom, the distance, d, from the origin of the phase-space source to the isocentre, and a polar angle, $\theta$, rotating this distance vector about the $\mathrm{X}$-axis of the phantom. The polar angle is defined such that at 0 degrees the source points along the $\mathrm{Z}$-axis (i.e. from the top of the phantom), while $\theta=90$ degrees points the source along the negative Y-axis (i.e. from the back of the phantom). During a simulation, azimuthal rotation of the source (actually, the isocentre-source distance vector) about the Z-axis is obtained by randomly sampling azimuthal angles, $\phi$, in the range 0-360 degrees for each new primary history simulated. The azimuthal angle is defined relative to the phantom Y-axis. Azimuthal rotation is used to simulate rotation of the CBCT source-detector about the patient during a scan.

The existing FAX06/MAX06 user code also required modification to allow scoring of total, RBM and BSC dose on a voxel-by-voxel basis. Since the phantom contains so many voxels, it is impractical to consider dose in every voxel. Therefore, an input is introduced allowing the user to specify $\mathrm{X}, \mathrm{Y}$ and $\mathrm{Z}$ planes defining a dose region. The location of the dose region will depend upon the particular scan being simulated. Total dose is scored in every voxel in the dose region but in none outside of it. Voxel- 


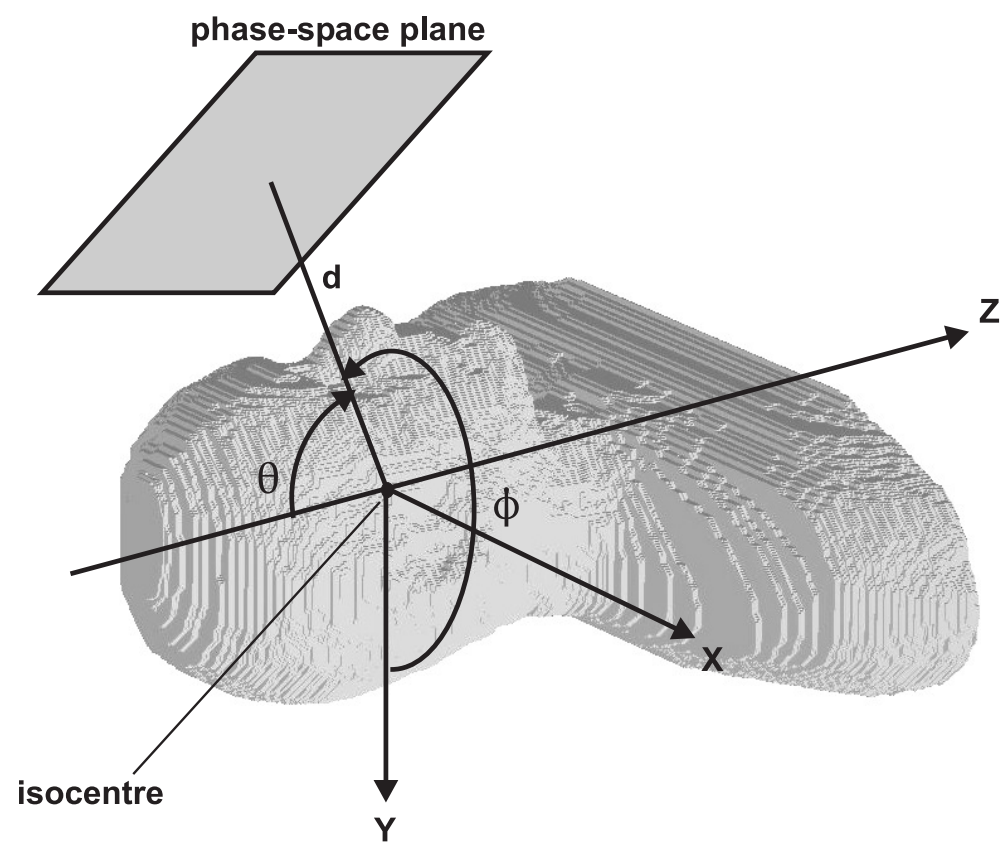

Figure 6.2: A schematic of the phase-space source in the FAX06/MAX06 user code added for this study. The user specifies the isocentre point, the distance from the origin of the phase-space plane to the isocentre, $\mathrm{d}$, and the polar angle, $\theta$, between the negative Z-axis and $\mathrm{d}$. During the simulation, random azimuthal angles, $\phi$, in the range 0-360 degrees are chosen for each new primary history simulated to simulate a 360 degree CBCT scan. Azimuthal angles are defined relative to the Y-axis. Note that a simulation with $\theta=0$ points the source in the Z-direction.

by-voxel RBM and BSC doses must also be scored separately, but since not every voxel in a dose region has micro voxels containing RBM/BSC, significant memory can be saved by using smaller scoring arrays for the RBM and BSC doses. This requires an additional "index" array that maps the index of every voxel containing RBM/BSC in the dose region to its counterpart in the reduced RBM and BSC dose arrays. In the simulated chest scan of FAX06 described below, the dose region contains 20,345,702 voxels, while only 479,502 contain any RBM/BSC micro voxels.

Doses calculated using the modified code can either be stored in an ASCII format used by the DOSXYZnrc user code[29], called .3ddose format, or in a much smaller binary (.bindose) format. The .3ddose format allows isodose contours to be displayed using the dosxyz_show [30] tool distributed with BEAMnrc/DOSXYZnrc. Unfortunately, . 3ddose files for such large dose arrays can be prohibitively large (especially if running many parallel jobs). Thus, it is preferable to use the much smaller . bindose format. The only disadvantage of this format is that geometrical information about the voxel size and number of voxels in each dimension is not stored, requiring that this information be input to any code analyzing the . bindose files or that the FAX06/MAX06 user code itself be used to re-read and analyze 
these files.

\subsection{The CBCT phase-space source}

The phase-space source used in these simulations was provided by Ding[31] and is the same as that used by Ding et al[14] in a study of patient dose during a simulated head and neck scan. It was generated by a BEAMnrc simulation of a Varian OBI CBCT system, which is integrated into a Trilogy ${ }^{T M}$ accelerator. Dose calculation results from other $\mathrm{kV} \mathrm{CBCT}$ units on the market are expected to be similar. The OBI system is capable of generating beams with photon spectra in the range $40-125 \mathrm{kVp}$. To acquire the phase-space file used in this study, Ding et al[14] simulated the default settings of the OBI system which produce a beam with a $125 \mathrm{kVp}$ photon spectrum (tube current $=80 \mathrm{~mA}$, exposure time $=25 \mathrm{~ms} /$ frame). The CBCT simulation was run in half-fan mode, which is used when the imaged target is greater than 24 $\mathrm{cm}$ in diameter. The combination of blade settings and filtration used in half-fan mode result in an $\mathrm{x}$-ray field that is asymmetric in X, with a more gradual fall-off and longer tail in the positive $\mathrm{X}$ direction. Since the source is rotated through 360 azimuthal degrees in the simulations in this study, it is unlikely that this asymmetry will have any effect. In order to increase the efficiency of the CBCT simulation, Ding et al[14] used directional bremsstrahlung splitting[32] with a splitting number of 2000. The phase-space data was acquired at a plane $20 \mathrm{~cm}$ from the patient isocentre using a simulated source-to-isocentre distance of $100 \mathrm{~cm}$. The beam field at the phase-space plane is approximately $10 \mathrm{~cm} \times 20 \mathrm{~cm}$, not including the tail in the $+\mathrm{X}$ direction. There are total of $66.1 \times 10^{6}$ particles in the the phase-space file, representing $1.3 \times 10^{9}$ primary histories incident on the $\mathrm{x}$-ray target.

In the simulated head and neck scans in this study, the distance between the phase-space source plane and the isocentre is $20 \mathrm{~cm}$, the same distance at which the phase-space data was acquired by Ding et al[14]. However, in the chest and pelvis scans, this distance must be increased to $30 \mathrm{~cm}$ to allow the phase-space plane to clear all phantom anatomy. Since the same phase-space source (described above) is used for all scans, increasing the distance between the phase-space plane and the isocentre by $10 \mathrm{~cm}$ is equivalent to increasing the source-to-isocentre distance to $110 \mathrm{~cm}$. To renormalize results to a sourceto-isocentre distance of $100 \mathrm{~cm}$, all doses from the simulated chest and pelvis scans are multiplied by a factor of $(110 / 100)^{2}$.

\subsection{Calibration of Monte Carlo dose}

Dose output from the FAX06/MAX06 simulations (and, indeed, all EGSnrc-based user codes) is in terms of Gy/incident primary history. In order to convert this into the more meaningful quantity of Gy, Ding et al[14] introduce a Monte Carlo calibration factor, $f_{M C c a l}$, defined as the ratio of measured to Monte Carlocalculated dose at a known point. Once calculated for a phase-space source derived from a simulated $\mathrm{CBCT}$ scan, $f_{M C \text { cal }}$ is valid for all dose calculations using that phase-space source, and the Monte Carlo 
estimated actual dose, $\mathrm{D}_{M C}$, at any point is given by:

$$
D_{M C}=f_{M C c a l} D_{M C c a l}
$$

where $D_{M C \text { cal }}$ is the Monte Carlo calculated dose at the point in Gy/incident particle. The value of $f_{M C \text { cal }}$ provided by Ding[31] for the CBCT phase-space source used in this study is $5.28 \times 10^{28} \mathrm{cGy} / \mathrm{Gy} /$ particle. This value of $f_{M C \text { cal }}$ implicitly takes into account the CBCT parameters simulated: $125 \mathrm{kVp}$ photon spectrum, tube current $=80 \mathrm{~mA}$, exposure time $=25 \mathrm{~ms} /$ frame.

\subsection{Simulations}

All FAX06/MAX06 simulations are performed with electron transport cut-off energies of $200 \mathrm{keV}$ outside skeletal tissue and $5 \mathrm{keV}$ inside the skeleton. The range of $5 \mathrm{keV}$ electrons in soft tissue is $\sim 1 \mu \mathrm{m}$, which should be adequate for accurate transport in the 17.65 cubic $\mu \mathrm{m}$ RBM micro voxels and $10 \mu \mathrm{m}$ thick BSC layer. Photon cut-off energy is $2 \mathrm{keV}$ everywhere in the phantom. Simulations are divided into 100 parallel jobs run on a cluster of Intel Xeon 5460 CPU's. Each job uses a different partition of the phase-space source. Thus, each job makes use of $66.1 \times 10^{6} / 100=661,000$ particles in the phase-space source.

Other simulation parameters for the head and neck, chest, and pelvis scans of FAX06 and MAX06 are given in Table 6.1. The table also shows the average uncertainties obtained on all total, RBM and BSC doses greater than $50 \%$ of their respective maxima. In all simulations, the isocentre is chosen to be approximately at the centre of the volume of interest with the source-to-isocentre distance of $20 \mathrm{~cm}$ for the head and neck scans and $30 \mathrm{~cm}$ for the chest and pelvis scans. In the case of the simulated chest scan of FAX06, an additional simulation is performed with the $\mathrm{X}$ and $\mathrm{Y}$ position of the isocentre shifted to the back of the right lung (to simulate treatment in this location) to determine the effects of an isocentre that is not in the centre of the patient. Note that for this off-centre scan the source-to-isocentre distance must be increased to $40 \mathrm{~cm}$ for the phase space plane to clear all patient geometry.

\begin{tabular}{|c|c|c|c|c|c|c|c|}
\hline scan & phantom & isocentre Z & NCASE (prim. hist.) & CPU time & \multicolumn{2}{|c|}{$\sigma_{D>0.5 D_{\max }}(\%)$} \\
& & $(\mathrm{cm})$ & $\left(\times 10^{9}\right)$ & $(\mathrm{hrs})$ & total & RBM & BSC \\
\hline head & FAX06 & 9.5 & $192.5(3,786)$ & 3,790 & 0.28 & 1.1 & 2.3 \\
and neck & MAX06 & 10.8 & $210(4,130)$ & 4,609 & 0.28 & 1.1 & 2.3 \\
\hline chest & FAX06 & 40.2 & $60(1,180)$ & 1,471 & 0.52 & 1.5 & 5.5 \\
& MAX06 & 45.0 & $48.5(953.9)$ & 1,174 & 0.60 & 1.7 & 6.3 \\
\hline pelvis & FAX06 & 75.4 & $40(786.7)$ & 1,173 & 0.73 & 2.1 & 7.7 \\
& MAX06 & 88.9 & $43(845.7)$ & 1,217 & 0.69 & 1.9 & 6.9 \\
\hline
\end{tabular}

Table 6.1: Simulation parameters for the simulated CBCT head and neck, chest and pelvis scans of FAX06 (female phantom) and MAX06 (male phantom). For the number of incident particles (NCASE), the number of primary histories represented is shown in brackets. Isocentre $(\mathrm{X}, \mathrm{Y})$ values are always on the centreline of the phantom $(28.6 \mathrm{~cm}, 13.4 \mathrm{~cm})$. Also shown are the average $\%$ uncertainties obtained on all total, RBM and BSC doses $>50 \%$ of their respective maxima $\left(\sigma_{D>0.5} D_{\max }\right)$. Note that no. of incident particles and CPU time represent the total values. In practice, these are divided over 100 parallel jobs. 
Note that the number of incident particles in all simulations means that phase-space data is recycled many times (3,177 times in the case of the simulated head and neck scan of MAX06). Since each primary history is incident from a different azimuthal angle, this large amount of data recycling is not expected to have a significant impact on dose uncertainties.

\subsection{Results and Discussion}

Cumulative dose volume histograms (DVH's) for the simulated head and neck scans are shown in Figure 6.3 for the FAX06 (a) and MAX06 (b) phantoms. Equivalent results are shown for the simulated chest scans in Figure 6.4 and for the simulated pelvis scans in Figure 6.5.

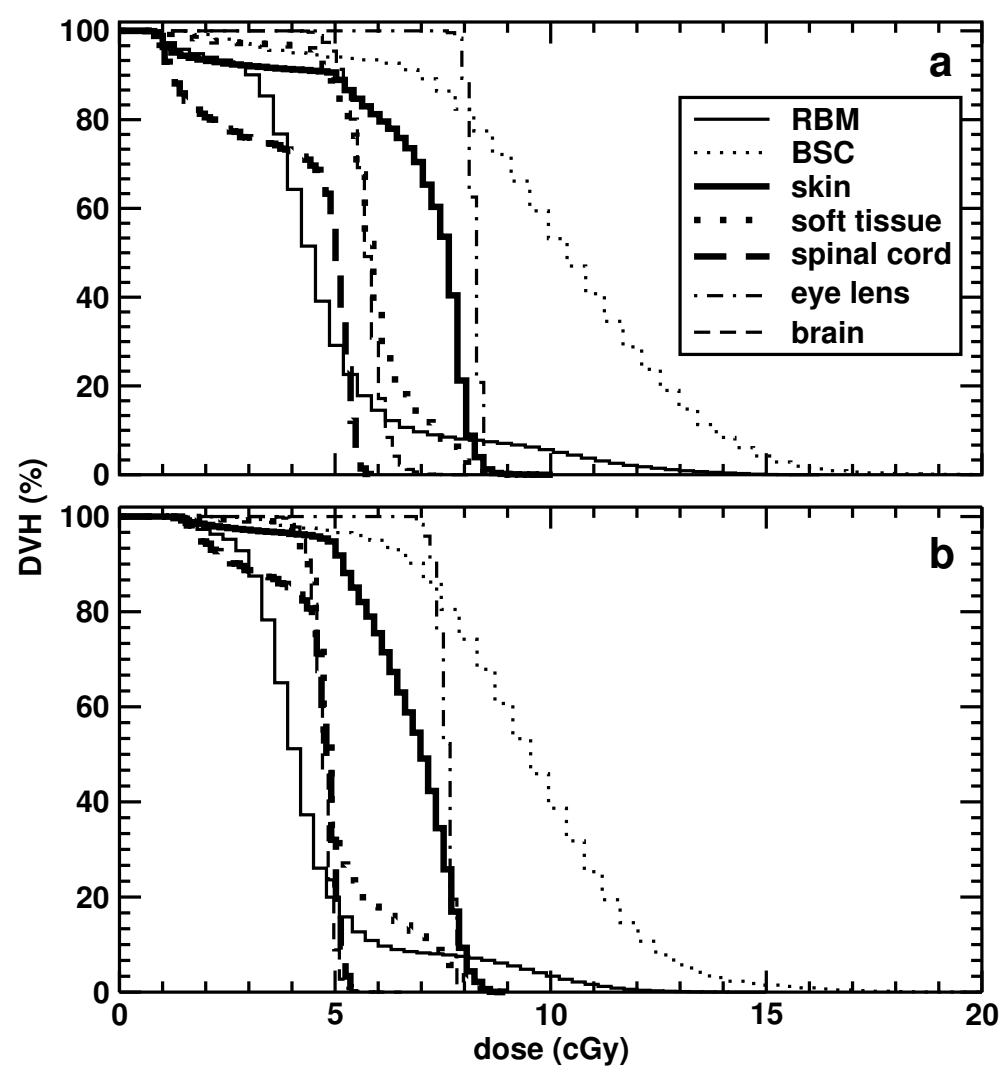

Figure 6.3: Cumulative DVH's for simulated head and neck scans of the FAX06 (a) and MAX06 (b) phantoms. In addition to showing the DVH's for all soft tissue, DVH's are also shown for brain (thin dashed line) and eye lens (thin dot-dashed line).

All figures show dose to RBM, BSC, skin, soft tissue and, where relevant, the spinal chord. Dose to certain organs at risk is resolved from the total soft tissue dose (e.g. dose to brain in Figure 6.3 and 


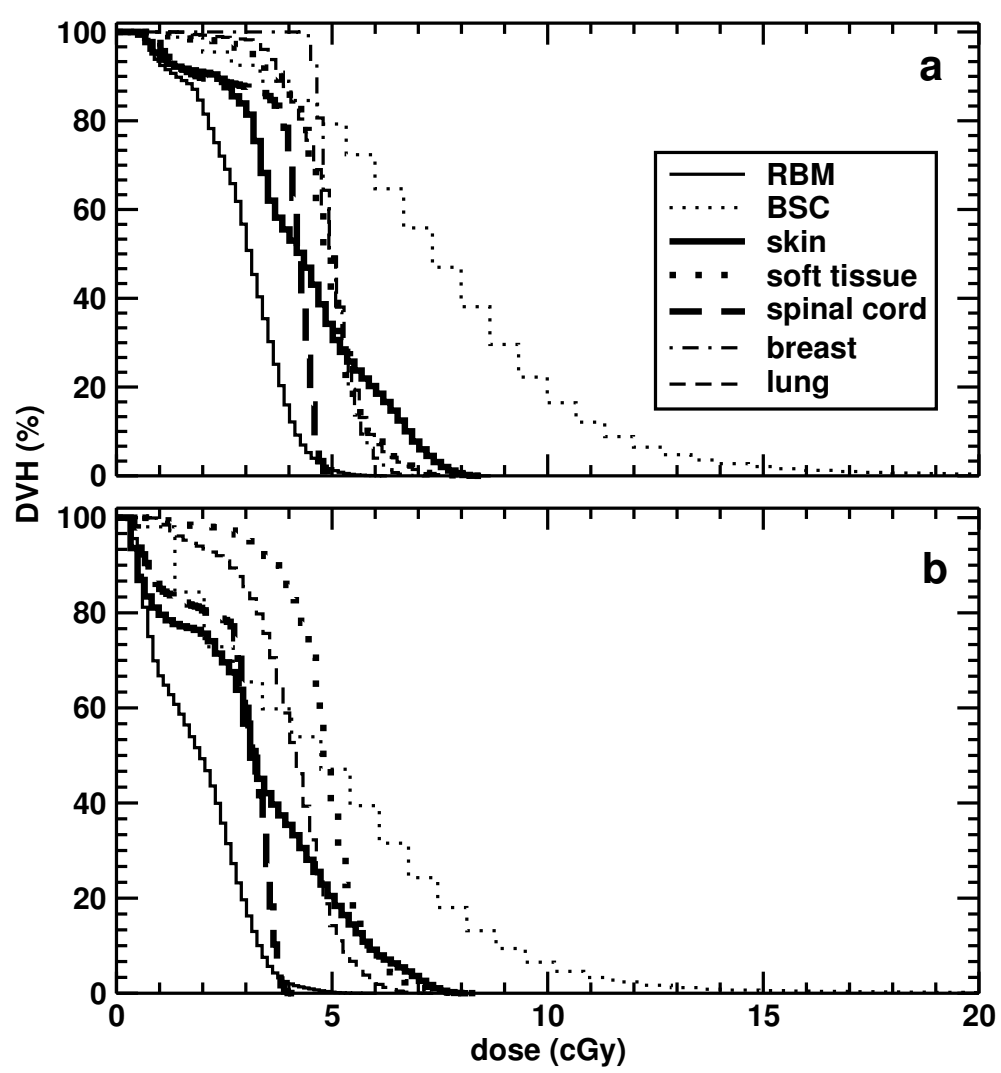

Figure 6.4: Cumulative DVH's for simulated chest scans of the FAX06 (a) and MAX06 (b) phantoms. Dose to lung (thin dashed line) and, in the case of FAX06, breast (thin dot-dashed line) are resolved from the dose to soft tissue.

dose to prostate/ovaries in Figure 6.5). For soft tissues, skin, the spinal chord, and lung (in the case of the simulated chest scans), doses are in good agreement with the range of doses calculated by Ding et $\mathrm{al}[14]$ using the phase-space data from a simulated CBCT scan as a source on a phantom derived from patient CT data in DOSXYZnrc. In addition, the DVH's for soft tissue and organs at risk also show good qualitative and quantitative agreement with DVH's calculated for adult patients in a more recent Monte Carlo study by Ding and Coffey[18], also using DOSXYZnrc phantoms based on patient CT data.

In most cases, the doses calculated in the male phantom (MAX06) are less than those calculated for the same organs in the female phantom (FAX06). The most significant example of this is observed in the simulated head and neck scan (Figure 6.3), where the average dose to soft tissue in FAX06 (5.9 cGy) is $20 \%$ greater than the average dose to soft tissue in MAX06 (4.9 cGy). Similar differences are seen between doses to brain and eye lens in this scan. These differences are due to the larger anatomical size of the MAX06 phantom and the associated attenuation effect. 


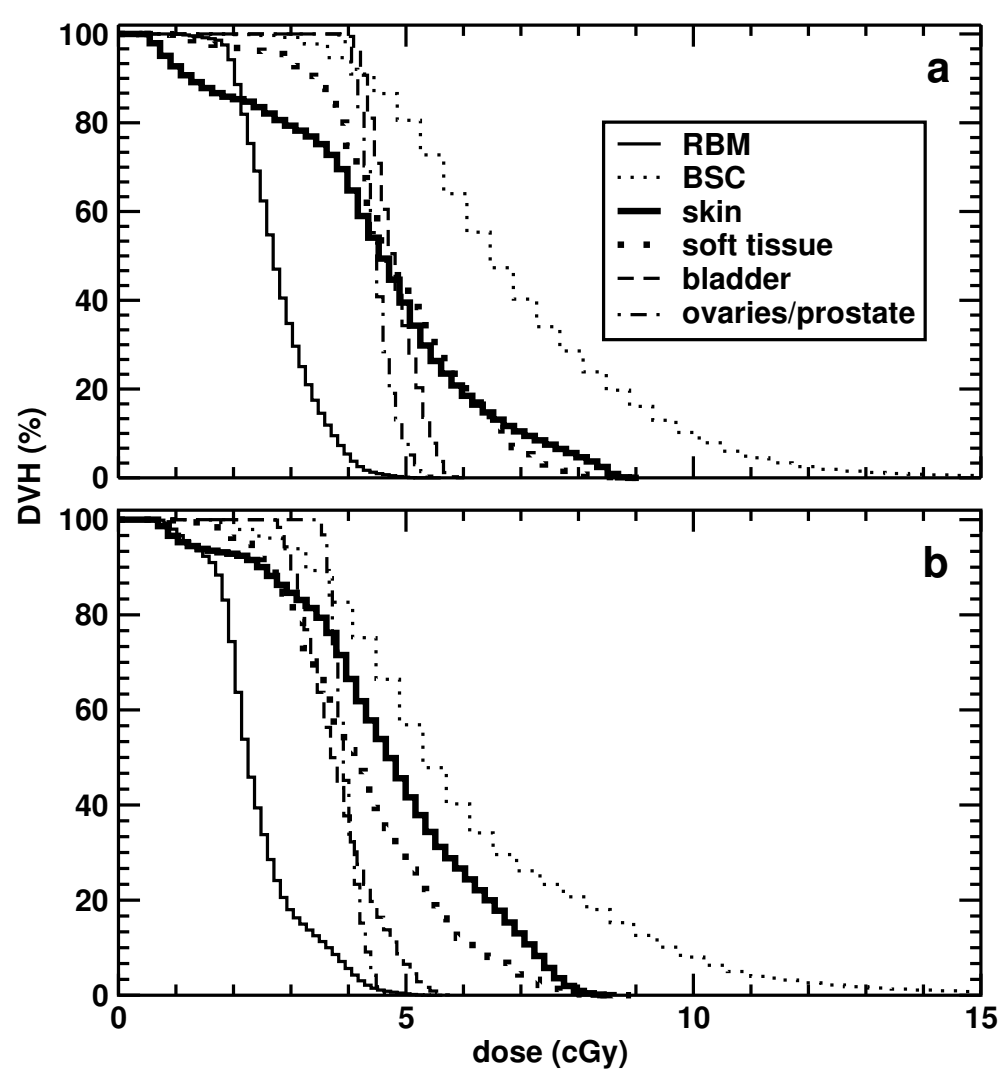

Figure 6.5: Cumulative DVH's for simulated pelvis scans of the FAX06 (a) and MAX06 (b) phantoms. Dose to bladder (thin dashed line) and dose to ovaries (a) or prostate (b) (thin dot-dashed line) are also shown.

In terms of sensitive tissue within the skeletal system, in almost all cases RBM receives a lower average dose than other organs at risk. For example, in the simulated head and neck scans (Figure 6.3), the average RBM dose is $\sim 80 \%$ of the average dose to brain and $\sim 50 \%$ of the average dose to eye lens. The simulated head scans are unique in that a small fraction of the RBM ( 10\%) receives doses in excess of $10 \mathrm{cGy}$, which is higher than the maximum dose to skin in these scans. The BSC, on the other hand, almost always receive a higher average dose than other sensitive organs. For example, in the simulated head and neck scan in Figure 6.3, the average dose to BSC is $~ 25 \%$ higher than the average dose to eye lens, and in the simulated pelvis scans (Figure 6.5) the average BSC dose is $44 \%$ greater than the average dose to ovaries (FAX06) and 35\% greater than the average dose to prostate (MAX06). The only exception is in the simulated chest scan of MAX06 (Figure 6.4b) where the average dose to BSC and to all soft tissues are approximately equal. Note that for the simulated chest scan of FAX06 (Figure 6.4a), however, the average BSC dose is $\sim 50 \%$ greater than the average dose to soft tissues. In all cases, some fraction (10-20\% in chest 
and pelvis scans, $40-50 \%$ in head scans) of the BSC receives doses > $10 \mathrm{cGy}$.

Table 6.2 shows the average RBM and BSC doses at different locations in each simulated scan (average doses for all RBM and BSC are shown in bold). The highest average RBM and BSC doses occur in structures surrounding the isocentre of the scan (cranium in the head and neck scan, ribs in the chest scan, pelvis in the pelvis scan) and in the spine (cervical, thoracic and lumbar regions). In the case of the pelvis scan, the average BSC dose in lumbar spine exceeds that in the pelvis BSC by $23 \%$ in the female phantom and 70\% in the male phantom. Extremities (arms and legs) contribute less to the overall RBM and BSC doses, with the one exception of the upper legs in the male phantom, in which the average doses to RBM and BSC are similar to those in the pelvis.

\begin{tabular}{|c|c|c|c|c|c|}
\hline scan & location & \multicolumn{2}{|c|}{ average RBM dose (cGy) } & \multicolumn{2}{c|}{ average BSC dose (cGy) } \\
& & FAX06 & MAX06 & FAX06 & MAX06 \\
\hline head & cranium & 5.0 & 4.2 & 10.8 & 9.8 \\
and neck & cervical spine & 4.0 & 3.9 & 9.1 & 9.1 \\
& all & $\mathbf{4 . 6}$ & $\mathbf{4 . 2}$ & $\mathbf{1 0 . 4}$ & $\mathbf{9 . 5}$ \\
\hline chest & ribs & 3.2 & 2.4 & 7.7 & 6.1 \\
& scapula & 1.9 & 1.0 & 4.7 & 2.2 \\
& thoracic spine & 3.4 & 2.6 & 8.1 & 5.9 \\
& upper arms & 1.9 & 0.9 & 4.9 & 2.6 \\
& all & $\mathbf{3 . 1}$ & $\mathbf{2 . 0}$ & $\mathbf{7 . 4}$ & $\mathbf{4 . 8}$ \\
\hline pelvis & pelvis & 2.9 & 2.2 & 6.6 & 5.0 \\
& lumbar spine & 3.3 & 2.0 & 8.1 & 8.5 \\
& upper legs & 2.2 & 2.0 & 5.3 & 5.1 \\
& all & $\mathbf{2 . 7}$ & $\mathbf{2 . 2}$ & $\mathbf{6 . 5}$ & $\mathbf{5 . 2}$ \\
\hline
\end{tabular}

Table 6.2: Average RBM and BSC doses at various locations for the head and neck, chest and pelvis scans. Results are shown for the female (FAX06) and male (MAX06) phantoms. The average doses for all RBM and BSC in each scan is also shown in bold.

Figure 6.6 shows the cumulative DVH's for RBM and BSC in the simulated chest scan for FAX06 with the isocentre in the centre of the volume of interest (thin lines-same as Figure 6.4 above) and with the $\mathrm{X}$ and $\mathrm{Y}$ positions of the isocentre shifted to put it near the back of the right lung (thick lines). The exact position of the isocentre has little effect on the RBM and BSC doses which are the focus of this study.

\subsection{Conclusions}

Voxelized human female (FAX06) and male (MAX06) phantoms, developed by Kramer et al[21], have been used with phase-space data from a simulated CBCT scanner (Varian OBI) to successfully calculate dose in organs at risk during simulated head and neck, chest and pelvis scans. The phantoms allow separation of dose to spongiosa (defined as a mixture of bone marrow and trabecular bone) to be separated into dose to red bone marrow (RBM) and bone surface cells (BSC). Significant modifications to the EGSnrc-based Monte Carlo codes in which FAX06/MAX06 are implemented were required to allow use of the CBCT phase-space data as a source and to allow dose scoring on a voxel-by-voxel basis. Doses and 


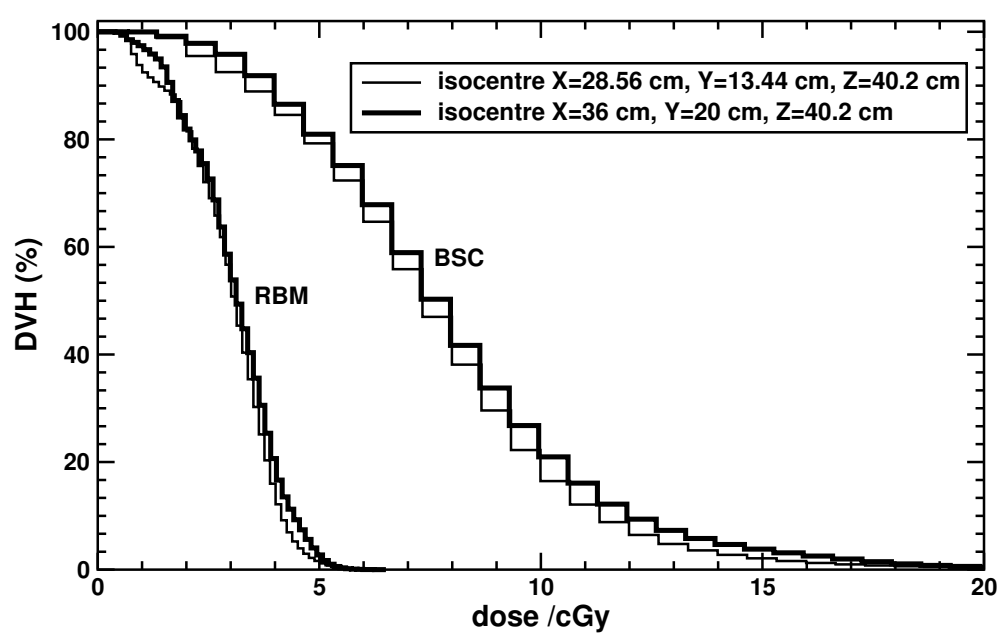

Figure 6.6: Cumulative DVH's for red bone marrow (RBM) and bone surface cells (BSC) from simulated chest scans of FAX06 with two different isocentre positions. Thin lines show DVH's with the isocentre in the centre of the volume of interest (i.e. same as in Figure 6.4a), while thick lines show results with $\mathrm{X}$ and $\mathrm{Y}$ position of the isocentre near the back of the right lung. Note that for the off-centre scan, the distance from the source to isocentre is increased to $40 \mathrm{~cm}$ to allow the phase space plane to clear all patient anatomy.

dose volume histograms calculated in FAX06 and MAX06 are in good agreement with those calculated by Ding et al[14] (doses) and Ding and Coffey[18] (DVH's) using phantoms based on actual CT data and are, therefore, expected to reflect doses received during typical CBCT scans in the clinic.

Ding et al[14] and Ding and Coffey[18] observed that the dose to bone was 2-4 times greater than dose to soft tissue. With the current study, it is possible to conclude that this elevated bone dose results in elevated dose to the BSC. The average doses to BSC calculated in this study are up to $46 \%$ higher than the next highest average dose to an organ at risk. In addition, significant fractions of the BSC receive doses higher than the maximum dose to all other soft-tissue. The dose to the other organ at risk within the bone, RBM, is roughly half the dose to BSC with an average dose that is less than the average dose to soft tissue in all scans simulated.

In all simulated scans, the calculated BSC dose is greater in the female phantom (FAX06) than in the male phantom (MAX06). As mentioned above, this is due to the larger anatomical size of MAX06. Specifically, the male phantom has a thicker layer of cortical bone surrounding the spongiosa, and this provides greater shielding for the BSC. Extrapolating the results of this study to pediatric patients, in which the cortical layer is thinner than in an adult female, the dose to BSC is expected to be even higher. Clearly, this is a concern and an area for future research.

The mechanism by which BSC receives an elevated dose is well-understood and described in some detail in references[21,28]. Briefly, the additional dose is due to low-energy electrons liberated in photo- 
electric events in trabecular bone. These electrons have a range of a few tens of $\mu \mathrm{m}$ or less and, therefore, deposit most of their dose in the BSC layer. Dose in RBM, by contrast, results from electrons set in motion within the RBM and is lower than the dose in other soft tissues due to the shielding effect of trabecular bone.

Ding et al[14] have pointed out that the additional dose due to CBCT represents only $2 \%$ (for soft tissue) to $10 \%$ (for bone) of the typical prescribed treatment dose. However, as Ding and Coffey[18] note, the CBCT dose is delivered throughout the imaging volume, which includes soft tissue and bone beyond the treatment volume, and imaging occurs with every treatment (typically 25-35 times), so the additional dose occurs on a repeated basis. When these factors are considered together with the results of this study, indicating that a significant portion of the additional bone dose occurs in the BSC, then the added dose from CBCT becomes an important consideration in its use in image-guided radiotherapy.

\subsection{Bibliography}

[1] D. A. Jaffray, D. G. Drake, M. Moreau, A. A. Martinez, and J. W. Wong. A radiographic and tomographic imaging system integrated into a medical linear accelerator for localization of bone and soft-tissue targets. Int J Radiat Oncol Biol Phys, 45:773 - 789, 1999.

[2] D. A. Jaffray and J. H. Siewerdsen. one-beam computed tomography with a flat-panel imager: initial performance characterization. Med Phys, 27:1311 - 1323, 2000.

[3] D. A. Jaffray, J. H. Siewerdsen, J. W. Wong, and A. A. Martinez. Flat-panel cone-beam computed tomography for image-guided radiation therapy. Int J Radiat Oncol Biol Phys, 53:1337 - 1349, 2002.

[4] M. B. Sharpe, D. J. Moseley, T. G. Purdie, M. Islam, J. H. Siewerdsen, and D. A. Jaffray. The stability of mechanical calibration for a $\mathrm{kV}$ cone beam co mputed tomography system integrated with linear accelerator. Med. Phys., 33:136 - 144, 2006.

[5] A. S. Shiu, E. L. Chang, J. S. Ye, M. Lii, L. D. Rhines, E. Mendel, J. Weinberg, S. Singh, M. H. Maor, R. Mohan, and J. D. Cox. Near simultaneous computed tomography image-guided stereotactic spinal radiotherapy: an emerging paradigm for achieving true stereotaxy. Int J Radiat Oncol Biol Phys, 57:605 - 613, 2003.

[6] J. H. Siewerdsen and D. A. Jaffray. Optimization of x-ray imaging geometry (with specific application to flat-panel cone-beam computed tomography). Med. Phys., 27:1903 - 1914, 2000.

[7] M. Uematsu, T. Fukui, A. Shioda, H. Tokumitsu, K. Takai, T. Kojima, Y. Asai, and S. Kusano. A dual computed tomography linear accelerator unit for stereotactic radiation therapy: a new approach without cranially fixated stereotactic frames. Int J Radiat Oncol Biol Phys, 35:587 - 592, 1996.

[8] K. M. Yenice, D. M. Lovelock, M. A. Hunt, W. R. Lutz, N. Fournier-Bidoz, C. H. Hua, J. Yamada, M. Bilsky, H. Lee, K. Pfaff, S. V. Spirou, and H. I. Amols. CT image-guided intensity-modulated 
therapy for paraspinal tumors using stereotactic immobilization. Int J Radiat Oncol Biol Phys, 55: $583-593,2003$.

[9] D. M. Duggan, G. X. Ding, 2nd C. W. Coffey, W. Kirby, D. E. Hallahan, A. Malcolm, and B. Lu. Deep-inspiration breath-hold kilovoltage cone-beam CT for setup of stereotactic body radiation therapy for lung tumors: Initial experience. Lung Cancer, 56:77 - 88, 2007.

[10] G. X. Ding, D. M. Duggan, C. W. Coffey, M. Deeley, D. E. Hallahan, A. Cmelak, and A. Malcolm. A study on adaptive IMRT treatment planning using kV cone-beam CT. Radiother Oncol, 85:116 $125,2007$.

[11] N. Wen, H. Guan, R. Hammoud, D. Pradhan, T. Nurushev, S. Li, and B. Movsas. Dose delivered from Varian's CBCT to patients receiving IMRT for prostate cancer. Phys. Med. Biol., 52:2267-2276, 2007.

[12] O. Gayou, D. S. Parda, M. Johnson, and M. Miften. Patient dose and image quality from megavoltage cone beam computed tomography imaging. Med. Phys., 34:499 - 506, 2007.

[13] M. K. Islam, T. G. Purdie, B. D. Norrlinger, H. Alasti, D. J. Moseley, M. B. Sharpe, J. H. Siewerdsen, and D. A. Jaffray. Patient dose from kilovoltage cone beam computed tomography imaging in radiation therapy. Med. Phys., 33:1573-1582, 2006.

[14] George X. Ding, Dennis M. Duggan, and Charles W. Coffey. Accurate patient dosimetry of kilovoltage cone-beam CT in radiation therapy. Med. Phys., 35:1135-1144, 2008. http://link . aip. org/link/?MPH/35/1135/1.

[15] J. H. Hubbell and S. M. Seltzer. Tables of X-Ray Mass Attenuation Coefficients and Mass EnergyAbsorption Coefficients $1 \mathrm{keV}$ to $20 \mathrm{MeV}$ for Elements Z $=1$ to 92 and 48 Additional Substances of Dosimetric Interest. Technical Report NISTIR 5632, NIST, Gaithersburg, MD 20899, 1995.

[16] J. H. Hubbell. Photon Mass Attenuation and Energy-absorption Coefficients from $1 \mathrm{keV}$ to $20 \mathrm{MeV}$. Int. J. Appl. Radiat. Isot., 33:1269 - 1290, 1982.

[17] S. M. Seltzer. Calculation of photon mass energy-transfer and mass energy-absorption coefficients (dosimetry application). Radiation Research, 136:147 - 170, 1993.

[18] G. X. Ding and C. W. Coffey. Radiation dose from kilovoltage cone beam computed tomography in an image-guided radiotherapy procedure. Int. J. Radiation Oncology Biol. Phys., 73:610 - 617, 2009.

[19] ICRP. Limits for Intakes of Radionuclides by Workers. ICRP Report 30, ICRP, Washington D.C., 1979.

[20] ICRP. Basic Anatomical and Physiological Data for use in Radiological Protection: The Skeleton. ICRP Report 70, ICRP, Washington D.C., 1995. 
[21] R Kramer, H J Khoury, J W Vieira, and V J M Lima. MAX06 and FAX06: update of two adult human phantoms for radiation protection dosimetry. Phys. Med. Biol., 51:3331-3346, 2006.

[22] I. Kawrakow. Accurate condensed history Monte Carlo simulation of electron transport. I. EGSnrc, the new EGS4 version. Med. Phys., 27:485 - 498, 2000.

[23] I. Kawrakow and D. W. O. Rogers. The EGSnrc Code System: Monte Carlo simulation of electron and photon transport. Technical Report PIRS-701 (4th printing), National Research Council of Canada, Ottawa, Canada, 2003.

[24] D. W. O. Rogers, B. Walters, and I. Kawrakow. BEAMnrc Users Manual. NRC Report PIRS 509(a)revI, 2005.

[25] ICRP. Basic Anatomical and Physiological Data for Use in Radiological Protection: Reference Values. Annals of the ICRP 89, ICRP, Washington D.C., 2002.

[26] ICRU. Tissue Substitutes in Radiation Dosimetry and Measurements. ICRU Report 44, ICRU, Washington D.C., 1989.

[27] R Kramer, H J Khoury, J W Vieira, and I Kawrakow. Skeletal dosimetry in the MAX06 and the FAX06 phantoms for external exposure to photons based on vertebral 3D-microCT images. Phys. Med. Biol., 51:6265-6289, 2006.

[28] J W Vieira R Kramer, H J Khoury and I Kawrakow. Skeletal dosimetry for external exposure to photons based on $\mu \mathrm{CT}$ images of spongiosa from different bone sites. Phys. Med. Biol., 52:66976716, 2007. http://stacks.iop.org/0031-9155/52/6697.

[29] B. R. B. Walters, I. Kawrakow, and D. W. O. Rogers. DOSXYZnrc Users Manual. NRC Report PIRS $794(\operatorname{rev} B), 2005$.

[30] I. Kawrakow. The dose visualization tool dosxyz_show. NRC Report PIRS 0624, 1998.

[31] G. X. Ding. personal communication, march 3, 2008.

[32] Iwan Kawrakow, D. W. O Rogers, and B.R.B. Walters. Large efficiency improvements in BEAMnrc using directional bremsstrahlung splitting. Med. Phys., 31:2883 - 2898, 2004. 



\section{VII}

\section{Dose to medium vs dose to water as an estimator of dose to sensitive skeletal tissue}

B. R. B. Walters, R. Kramer, I. Kawrakow Physics in Medicine and Biology July 2010: Vol. 55: 4535-4546 


\begin{abstract}
The purpose of this study is to determine whether dose to medium, $D_{m}$, or dose to water, $D_{w}$, provides a better estimate of the dose to the radiosensitive red bone marrow (RBM) and bone surface cells (BSC) in spongiosa, or cancellous bone. This is addressed in the larger context of the ongoing debate over whether $D_{m}$ or $D_{w}$ should be specified in Monte Carlo calculated radiotherapy treatment plans. The study uses voxelized, virtual human phantoms, FAX06/MAX06 (female/male), incorporated into an EGSnrc Monte Carlo code to perform Monte Carlo dose calculations during simulated irradiation by a $6 \mathrm{MV}$ photon beam from an Elekta SL25 accelerator. Head and neck, chest and pelvis irradiations are studied. FAX06/MAX06 include precise modeling of spongiosa based on $\mu \mathrm{CT}$ images, allowing dose to RBM and BSC to be resolved from the dose to bone. Significant modifications to the FAX06/MAX06 user codes are required for this study, including implementation of a BEAMnrc simulation source, scoring of $D_{w}$ simultaneously with $D_{m}$, and ability to substitute homogeneous spongiosa for spongiosa segmented into trabecular bone and bone marrow. Dose uncertainties of $\sim 1 \%$ (BSC, RBM) or $\sim 0.5 \%\left(D_{m}, D_{w}\right)$ are obtained after up to 5 days of simulations on 88 CPU's. Clinically-significant differences (> $5 \%$ ) between $D_{m}$ and $D_{w}$ are found only in cranial spongiosa, where the volume fraction of trabecular bone (TBVF) is high (55\%). However, for spongiosa locations where there is any significant difference between $D_{m}$ and $D_{w}$, comparisons of differential dose volume histograms (DVH's) and average doses show that $D_{w}$ provides a better overall estimate of dose to RBM and BSC. For example, in cranial spongiosa the average $D_{m}$ underestimates the average dose to sensitive tissue by at least $5 \%$, while average $D_{w}$ is within $\sim 1 \%$ of the average dose to sensitive tissue. Results from this study indicate that it is better to specify $D_{w}$ than $D_{m}$ in Monte Carlo calculated treatment plans, since $D_{w}$ provides a better estimate of dose to sensitive tissue within bone, the only location where the difference between $D_{w}$ than $D_{m}$ is likely to be clinically-significant.
\end{abstract}




\subsection{Introduction}

In recent years, Monte Carlo (MC) techniques have emerged as the most accurate method for calculating radiotherapy treatment plans. This has been fueled by ever-increasing computing speeds and the development of efficient Monte Carlo algorithms for simulating radiation transport, such as VMC [1, 2], xVMC [3, 4], VMC++ [5], MMC [6], or MCDOSE [7]. Currently, there are only a few Monte Carlo-based treatment planning systems on the market, however continued active development of $\mathrm{MC}$ techniques makes the widespread adoption of this method of treatment planning seem likely. This has raised the question of whether dose to medium, $D_{m}$, should replace dose to water, $D_{w}$, as the dose used in treatment plans, since the majority of $\mathrm{MC}$ algorithms report $D_{m}$, with conversion to $D_{w}$ requiring an extra step involving multiplying by the unrestricted stopping power ratio of water to medium, $s_{m}^{w}$. Compelling arguments for and against converting $D_{m}$ to $D_{w}$ were laid out in detail by [8], and a recent report of the AAPM Task Group 105 [9] investigating issues with the clinical implementation of MC-based treatment planning acknowledges this ongoing debate. Arguments in favour of changing to $D_{m}$-based protocols include: 1 ) the conversion from $D_{m}$ to $D_{w}$ adds an additional level of uncertainty due to uncertainties in computed stopping power ratios 2) $D_{m}$ is more likely to provide a better measure of biological response and 3) changing to $D_{m}$ will not have a clinically-significant impact on most treatment protocols, since most tissue of interest is similar to water. Meanwhile, arguments in favour of retaining $D_{w}$-based protocols include: 1) clinical experience in terms of tumour/tissue response is based on $D_{w}$ 2) dosimetry protocols are based on $D_{w}$ and so specifying $D_{w}$ allows traceability back to the calibration and 3) there is uncertainty in the medium type and composition when converting from CT numbers to media, potentially making the "medium" in $D_{m}$ unknown.

As indicated above, whether $D_{m}$ or $D_{w}$ is used to specify a treatment plan will not have a clinicallysignificant impact for most tissues of interest, which tend to have a composition close to water. One exception, however, is bone, in which the mass collision stopping power is lower than that of water by up to $15 \%$ in cortical bone $\left(\rho=1.92 \mathrm{~g} / \mathrm{cm}^{3}\right)$ and $3.5 \%$ in soft, cancellous bone, or spongiosa $\left(\rho=1.18 \mathrm{~g} / \mathrm{cm}^{3}\right)$. [10] have shown significant differences in isodose contours around bony anatomy for treatment plans using $D_{m}$ vs those using $D_{w}$. In a more detailed study of MC-calculated IMRT treatment plans, [11] showed that conversion to $D_{w}$ resulted in an increase in maximum and prescribed doses to the target volume (PTV) of 5.8\% and $2.9 \%$ respectively in head and neck treatments (6 MV photons) and $2 \%$ and $3.5 \%$ respectively in prostate treatments (18 MV photons). The greatest differences were observed when bony structures were involved in the PTV.

The purpose of this study is to investigate whether $D_{m}$ or $D_{w}$ provides a better estimate of the dose received by sensitive tissue within spongiosa during treatment by megavoltage photon beams. The spongiosa comprises bone marrow supported by a matrix of trabecular bone [12]. Radiosensitive tissue within the spongiosa are the haematopoietic stem cells, known as red bone marrow (RBM), which occupy a fraction of the bone marrow as a whole, and osteogenic cells on the surface of trabecular bone, called bone surface cells (BSC). The BSC cell layer is currently considered to have a thickness of $10 \mu \mathrm{m}$ [13]. Spongiosa occurs in various locations throughout the skeleton, with the trabecular bone volume frac- 
tion (TBVF) depending upon the location: in the rib cage TBVF=10\% $\left(\rho=1.0965 \mathrm{~g} / \mathrm{cm}^{3}\right)$, in the spine TBVF $=12 \%\left(\rho=1.1148 \mathrm{~g} / \mathrm{cm}^{3}\right)$, in the long bones TBVF=15\% $\left(\rho=1.1422 \mathrm{~g} / \mathrm{cm}^{3}\right)$, in the pelvis TBVF $=20 \%$ $\left(\rho=1.188 \mathrm{~g} / \mathrm{cm}^{3}\right)$, and in the mandible and cranium TBVF=55\% $\left(\rho=1.5082 \mathrm{~g} / \mathrm{cm}^{3}\right)$. With such variation in composition, the significance of the difference between $D_{m}$ and $D_{w}$ is likely to depend upon location.

This study makes use of the voxelized virtual human phantoms, FAX06 (adult female) and MAX06 (adult male), developed by [14] and incorporated into an EGSnrc [15] user code described in [16] and [17], to perform dose calculations in spongiosa resulting from simulated head and neck, chest, and pelvis irradiations. These virtual phantoms are particularly useful in that they allow the dose to RBM and BSC to be resolved from the overall dose to spongiosa. [18] made some modifications to the FAX06/MAX06 user code that are essential to this study, however, some additional modifications are required for the dose calculations presented here.

Section 7.2 gives a brief description of the FAX06/MAX06 virtual human phantoms and more detailed descriptions of the modifications required to the EGSnrc user code incorporating these phantoms to allow scoring of $D_{m}$ and $D_{w}$ in each voxel in a single simulation and also to allow the incorporation of a BEAMnrc shared library source for the purpose of simulating irradiations. Section 7.3 gives a description of the simulation parameters used in the virtual phantom dose calculations and in the BEAMnrc simulation source. The source used in this study is a $6 \mathrm{MV}$ photon beam $\left(10 \times 10 \mathrm{~cm}^{2}\right.$ field) from a simulated Elekta SL25 accelerator. This section includes the total number of histories run for each irradiation, uncertainties obtained, etc. Note that only results using FAX06 (the female phantom) are reported, since results using MAX06 are similar and, thus, largely redundant. Differential dose volume histograms (dDVH's) for RBM, BSC and homogeneous spongiosa evaluated using $D_{m}$ and $D_{w}$ are compared in Section 7.4 to ascertain whether $D_{m}$ or $D_{w}$ gives a better estimate of the dose to RBM and BSC. Concluding remarks appear in Section 7.5.

\subsection{The FAX06/MAX06 voxelized human phantoms}

The FAX06/MAX06 voxelized adult female/male phantoms were originally developed for radiation protection dosimetry and are described in detail by [14]. They were later incorporated into an EGSnrc [15] user code for the purpose of dose calculations [16, 17]. These phantoms are entire human bodies, reconstructed from segmented CT images and divided into $0.12 \times 0.12 \times 0.12 \mathrm{~cm}^{3}$ voxels throughout. Precise modeling of bone spongiosa was later introduced by [16] based on $\mu \mathrm{CT}$ images of actual spongiosa. Spongiosa voxels are divided into $(17.65 \mu \mathrm{m})^{3}$ microvoxels to form a micro-matrix of $68 \times 68 \times 68$ microvoxels in each spongiosa voxel. Each microvoxel is filled with either trabecular bone or bone marrow. Since it is computationally impractical to fill all $\sim 2$ million spongiosa voxels with a different micro-matrix for dose calculations, a repeating structure of eight different micro-matrices is applied on the fly during particle transport. The fraction of microvoxels containing trabecular bone must be equal to the trabecular bone volume fraction (TBVF) in the spongiosa being modeled (See Introduction above). Since spongiosa has five different TBVF's (10\%, 12\%, 15\%, 20\% and 55\%), depending on location in the body, FAX06/MAX06 carries data for $8 \times 5=40$ micro-matrices in all. 
Dose to RBM in FAX06/MAX06 is calculated by applying a "cellularity factor", related to the fraction of RBM in the bone marrow, to the dose deposited in microvoxels containing bone marrow. Dose to BSC, meanwhile, is computed by considering the fraction of an electron step in a bone marrow microvoxel that is within $10 \mu \mathrm{m}$ of a trabecular bone microvoxel [17]. The dose deposited in BSC by the electron is equal to this fraction times the dose deposited over the entire step.

Organ volumes and masses and tissue composition in FAX06/MAX06 are based on up-to-date values recommended by the International Commission on Radiological Protection [19] and the International Commission on Radiation Units [20]. The compositions of bone marrow and trabecular bone are shown in Table 7.1. These media are used to fill the microvoxels used to create the micro-matrices filling spongiosa voxels and, for this study, are also combined to create homogeneous spongiosa (described below) for the calculation of $\mathrm{D}_{m}$ and $\mathrm{D}_{w}$.

\begin{tabular}{ccc}
\hline element & $\begin{array}{c}\text { bone marrow } \\
(\%)\end{array}$ & $\begin{array}{c}\text { trabecular bone } \\
(\%)\end{array}$ \\
\hline $\mathrm{H}$ & 11.011 & 3.4 \\
$\mathrm{C}$ & 52.653 & 15.5 \\
$\mathrm{~N}$ & 2.102 & 4.2 \\
$\mathrm{O}$ & 33.834 & 43.5 \\
$\mathrm{Na}$ & 0.05 & 0.1 \\
$\mathrm{P}$ & 0.05 & 10.3 \\
$\mathrm{~S}$ & 0.15 & 0.3 \\
$\mathrm{Mg}$ & 0 & 0.2 \\
$\mathrm{~K}$ & 0.1 & 0 \\
$\mathrm{Fe}$ & 0.05 & 0 \\
$\mathrm{Ca}$ & 0 & 22.5 \\
$\rho\left(\mathrm{g} / \mathrm{cm}^{3}\right)$ & 1.005 & 1.92 \\
\hline
\end{tabular}

Table 7.1: Elemental composition by weight of bone marrow and trabecular bone as used in the FAX06/MAX06 phantoms.

Figure 7.1 shows a slice through the chest (at $\mathrm{Z}=40 \mathrm{~cm}$ ) of the MAX06 phantom. The anatomical structures are clearly visible in this cross-section, with the black portions indicating the location of the spongiosa in the spine, humerus and rib cage. The top image is one of the 8 micro-matrices (dimensions $0.12 \mathrm{~cm} \times 0.12 \mathrm{~cm} \times 0.12 \mathrm{~cm}$ ) used to model the spongiosa in the location indicated (the humerus, $\mathrm{TBVF}=15 \%)$.

A modification of the FAX06/MAX06 EGsnrc user code essential to this study is the ability to score total dose and dose to RBM and BSC on a voxel-by-voxel basis. This was first introduced by [18] in their study on additional dose due to cone beam CT imaging during image-guided radiotherapy. Since scoring dose in every voxel of FAX06 $(143,004,852$ voxels) or MAX06 $(153,738,108)$ is impractical, the user specifies a "dose region", which is a sub-volume of the phantom just encompassing the irradiated volume. In the case of, say, the simulated head and neck irradiation in FAX06 this contains 4,378,800 voxels. Total dose is scored in every voxel of the dose region, while smaller arrays of RBM and BSC doses are scored for only those voxels in the dose region containing microvoxels with either RBM or RBM and 


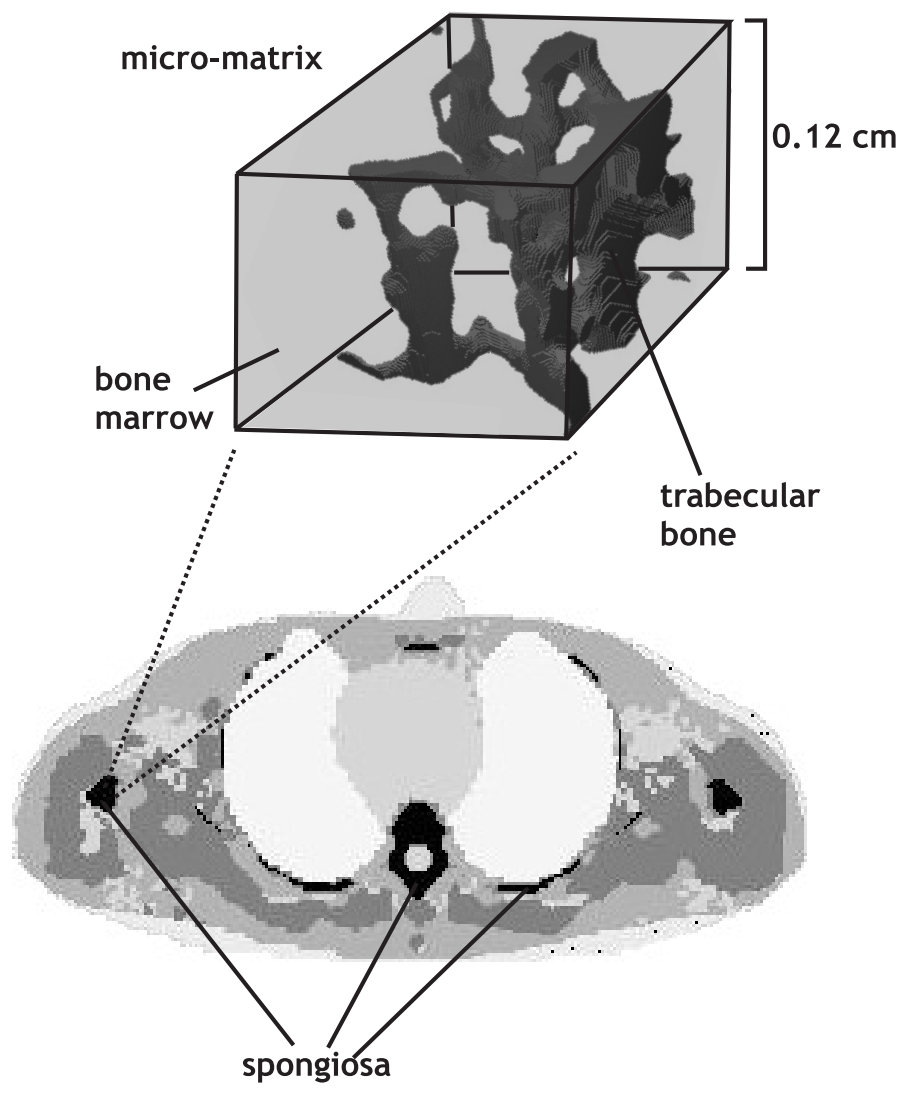

Figure 7.1: A slice (at $\mathrm{Z}=40 \mathrm{~cm}$ ) through the chest of the MAX06 phantom indicating the locations of spongiosa (black) in the spine, humerus and rib cage. The top image shows one of the 8 different micro-matrices $(0.12 \mathrm{~cm} \times 0.12 \mathrm{~cm} \times 0.12 \mathrm{~cm})$ used to model the spongiosa in the humerus (TBVF=15\%), indicating the trabecular bone and bone marrow portions. Each micro-matrix is comprised of $68 \times 68 \times 68$ micro voxels having dimensions 17.65 cubic $\mu \mathrm{m}$ and filled with either trabecular bone or bone marrow. Bone surface cells (BSC) are not modeled explicitly but for the purposes of dose deposition are considered to be a $10 \mu \mathrm{m}$ layer of bone marrow adjacent to trabecular bone.

BSC (this amounts to 204,036 voxels in the case of the simulated head and neck irradiation in FAX06).

\subsubsection{Additional modifications to FAX06/MAX06 user code for this study}

\subsubsection{BEAMnrc shared library source}

To facilitate the use of the BEAMnrc Monte Carlo code [21] for simulating the SL25 linac used in the dose calculations in this study, a BEAMnrc shared library source is implemented in the FAX06/MAX06 user code. Functionally, the shared library source as implemented here is similar to the BEAMnrc shared 
library sources available in other EGSnrc user codes [22-24]. The BEAMnrc accelerator model is compiled as a shared library and dynamically loaded into memory when a dose calculation is started. The FAX06/MAX06 code is the "driving" code, running new primary histories in the BEAMnrc shared library source as required. Particles from the source are sampled from the bottom of the accelerator (what would be the phase-space scoring plane in a stand-alone accelerator simulation).

The geometrical parameters for the shared library source in FAX06/MAX06 are similar to those for the phase space source in FAX06/MAX06 previously implemented by [18] and are illustrated in Figure 7.2. Note that the source plane in this figure corresponds to the phase space plane in the phase-space source. The main advantage of using a shared library source is that it obviates the need to store huge phase space files, which would exceed 1 terabyte for some of the calculations performed.

All simulated irradiations in this study are carried out with $\theta=90$ degrees (beam perpendicular to patient $\mathrm{Z}$-axis as in typical treatments) and with the gantry angle, $\phi$, rotated through 360 degrees.

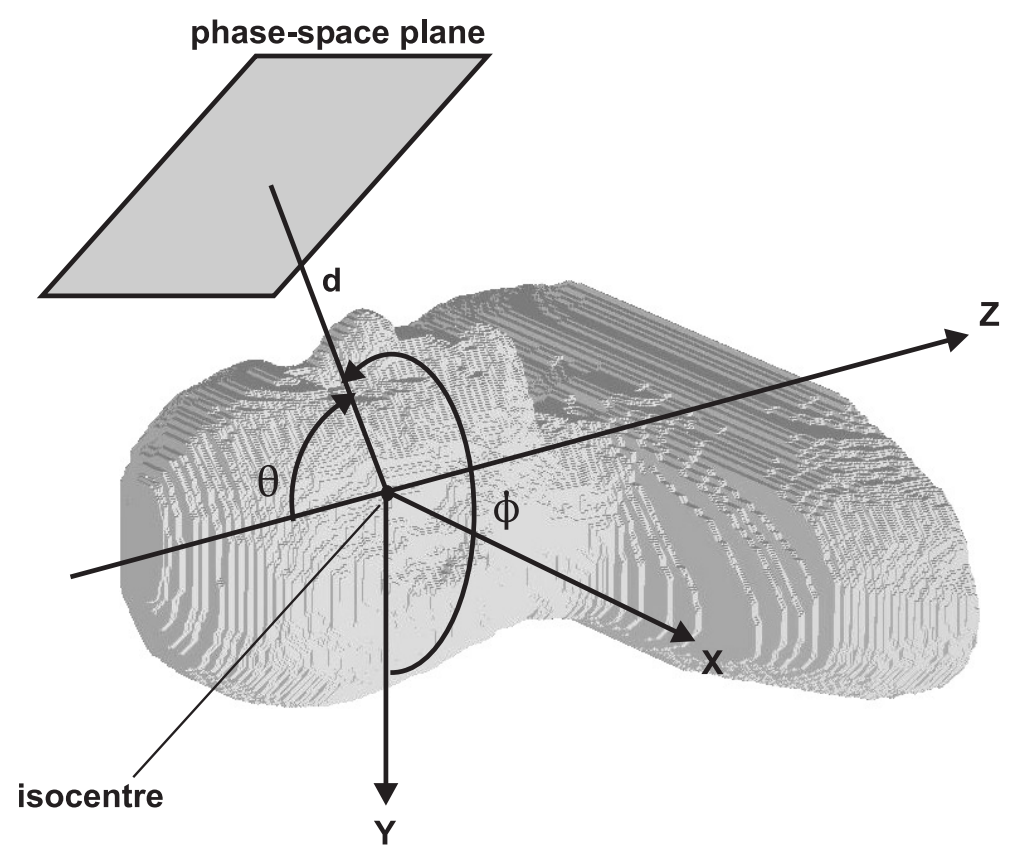

Figure 7.2: A schematic of the BEAMnrc simulation source in the FAX06/MAX06 user code implemented for this study. The user specifies the isocentre point, the distance from the origin of the source plane (where particle data is collected) to the isocentre, $d$, and the polar angle, $\theta$, between the negative $\mathrm{Z}$-axis and d. During the simulation, random azimuthal angles, $\phi$, in the range 0-360 degrees are chosen for each new primary history simulated to simulate a 360 degree CBCT scan. Azimuthal angles are defined relative to the Y-axis. Note that a simulation with $\theta=0$ points the source in the Z-direction. In this study, $\theta=90$ degrees for all simulations. 


\subsubsection{Calculation of $\mathbf{D}_{w}$}

The FAX06/MAX06 user code is modified to allow calculation of $\mathrm{D}_{m}$ and $\mathrm{D}_{w}$ during the same run by computing the restricted stopping power ratio of water to medium, $L_{m}^{w}$, on the fly for each energy deposition event. The energy deposited in water during the event, $\Delta E_{w}$, is then given by:

$$
\Delta E_{w}=L_{m}^{w} \Delta E_{m}
$$

where $\Delta E_{m}$ is the energy deposited in the medium, which is already calculated by the FAX06/MAX06 code.

The value of $L_{m}^{w}$ depends on the kinetic energy of the electron depositing energy and is evaluated using:

$$
L_{m}^{w}= \begin{cases}\frac{L_{w}\left(E_{\text {mid }}\right)}{L_{m}\left(E_{\text {mid }}\right)} & , \quad E>\Delta \\ \frac{S_{w}(\Delta)}{S_{m}(\Delta)} & , \quad E \leq \Delta\end{cases}
$$

where $E_{\text {mid }}$ is the mid-point energy of the electron step, $\Delta$ is the minimum kinetic energy for production of secondary electrons, and $S_{w}(\Delta)$ and $S_{m}(\Delta)$ are the unrestricted stopping powers for energy equal to $\Delta$ in water and medium, respectively. Note that for PEGS data [15], $\Delta=\mathrm{AE}-0.511 \mathrm{MeV}$. For steps which begin with $E>\Delta$ but end with $E<\Delta, L_{m}^{w}$ is calculated using both equations, with the upper result being multiplied by the fraction of the total kinetic energy above $\Delta$ and the lower result multiplied by the fraction below $\Delta$. For fluorescent photons created below the photon transport cutoff energy, PCUT, and depositing their energy on the spot, $L_{m}^{w}$ is evaluated as if they were electrons with kinetic energy $<\Delta$. Note that this method of determining $L_{m}^{w}$ is equivalent to that used by the EGSnrc user code, SPRRZnrc [23].

It is important to note that the method of evaluating $\mathrm{D}_{w}$ outlined here does not alter the particle transport in the medium in any way.

\subsubsection{Homogeneous spongiosa}

In order to allow comparison of dose in RBM and BSC to the $\mathrm{D}_{m}$ and $\mathrm{D}_{w}$ received by the spongiosa as a whole (which are the quantities computed in normal radiation treatment planning where the spongiosa structure is not resolved), an option is added to the FAX06/MAX06 user code to allow the replacement of bone marrow/trabecular bone micro-matrices in spongiosa voxels (described above) with homogeneous spongiosa. The composition of homogeneous spongiosa is determined using the compositions of bone marrow and trabecular bone shown in Table 7.1 above with the fraction of trabecular bone equal to the TBVF (see Introduction) at a given location. Thus, five distinct media, one for each TBVF, are created and used in the simulations. 


\subsection{Simulations}

\subsubsection{BEAMnrc simulation}

The BEAMnrc simulation of the $6 \mathrm{MV}$ photon beam $\left(10 \times 10 \mathrm{~cm}^{2}\right.$ field $)$ from an SL25 linac has the same geometrical and Monte Carlo transport parameters as those used by [25] and [26]. Geometrical parameters are based on the manufacturer's specifications. Directional bremsstrahlung splitting, DBS [27], with a bremsstrahlung splitting number of 1000 and including electron splitting below the flattening filter is used in this simulation. This provides optimum, or near-optimum efficiency for the BEAMnrc portion of the calculation. Note that contaminant electrons, although simulated in this study, are unlikely to contribute significantly to the bone doses discussed below.

\subsubsection{FAX06 Simulations}

Transport parameters in the FAX06 dose calculations are identical to those used by [18] in their calculations of additional bone dose due to CBCT imaging during IGRT. The electron transport cut-off energy (ECUT) is $200 \mathrm{keV}$ outside the bony anatomy and $5 \mathrm{keV}$ inside the skeleton. Note that the range of 5 $\mathrm{keV}$ in soft tissue is $\sim 1 \mu \mathrm{m}$, so this value of ECUT allows accurate transport in the 17.65 cubic $\mu \mathrm{m}$ RBM micro voxels and $10 \mu \mathrm{m}$-thick BSC layer. The photon cut-off energy (PCUT) is $2 \mathrm{keV}$ everywhere in the phantom. Simulations are divided into 88 parallel jobs and are run on a cluster of Intel Xeon 5460 CPU's.

Other simulation parameters for the head and neck, chest, and pelvis irradiations of FAX06 are given in Table 7.2. The table also shows the average uncertainties obtained on all total, RBM and BSC doses greater than $50 \%$ of their respective maxima. In all simulations, the isocentre is chosen to be approximately at the centre of the volume of interest with the source-to-isocentre distance of $20 \mathrm{~cm}$ for the head and neck irradiation and $30 \mathrm{~cm}$ for the chest and pelvis treatments.

\begin{tabular}{|c|c|c|c|c|c|c|c|c|}
\hline irradiation & isocentre Z & \multicolumn{2}{|c|}{ NCASE (prim. hist.) $\left(\times 10^{9}\right)$} & \multicolumn{2}{|c|}{ CPU time (hrs) } & \multicolumn{3}{|c|}{$\sigma_{D>0.5 D_{\max }}(\%)$} \\
& $(\mathrm{cm})$ & $\mathrm{MM}$ & $\mathrm{HS}$ & $\mathrm{MM}$ & HS & total & RBM & BSC \\
\hline head and neck & 9.5 & $23.6(14.2)$ & $26.4(15.8)$ & 3,934 & 2,427 & 0.48 & 0.86 & 1.06 \\
\hline chest & 40.2 & $33(19.8)$ & $39.6(23.8)$ & 5,210 & 4,105 & 0.45 & 0.54 & 0.99 \\
\hline pelvis & 75.4 & $45.8(27.5)$ & $40(24)$ & 9,822 & 4,129 & 0.47 & 0.58 & 0.98 \\
\hline
\end{tabular}

Table 7.2: Simulation parameters for the simulated head and neck, chest and pelvis irradiations of FAX06 (female phantom). Number of histories (NCASE) and CPU times are shown for simulations using bone marrow/trabecular bone micro-matrices in spongiosa voxels (MM-spongiosa with trabecular bone/bone marrow micro-matrices) and for simulations using homogeneous spongiosa (HS). For the number of incident particles (NCASE), the number of primary histories represented is shown in brackets. Isocentre $(\mathrm{X}, \mathrm{Y})$ values are always on the centreline of the phantom $(28.6 \mathrm{~cm}, 13.4 \mathrm{~cm})$. Average uncertainties (for doses $>0.5 \mathrm{D}_{\max }$ ) are shown for total (HS simulations only) and RBM and BSC (MM simultions only) doses. Note that no. of incident particles and CPU time represent the total values. In practice, these are divided over 88 parallel jobs. 


\subsection{Results and Discussion}

Differential dose volume histograms (DVH's) for RBM, BSC, $\mathrm{D}_{m}$ and $\mathrm{D}_{w}$ in FAX06 are shown for cranial spongiosa $(\mathrm{TBVF}=55 \%)$ during the simulated head and neck irradiation (Figure 7.3), for pelvis spongiosa (TBVF=20\%) during the simulated pelvis irradiation (Figure 7.4), and for thoracic spine spongiosa (TBVF=12\%) during the the simulated chest irradiation (Figure 7.5). These particular locations are selected because they allow comparison of DVH's over the entire range of TBVF values. Differential DVH's as opposed to their cumulative counterparts are chosen because they show simularities and differences between the dose distributions more clearly. Dose is given as Gy/incident history, which are the dose units returned by the FAX06/MAX06 user code. Conversion to absolute dose is possible given the correct calibration factor, however this will not affect the conclusions reached in this study. Average doses to RBM, BSC and average values of $\mathrm{D}_{m}$ and $\mathrm{D}_{w}$ at the various spongiosa locations, including cranium, pelvis and thoracic spine, are shown in Table 7.3.

Figure 7.3 shows that, while $\mathrm{D}_{w}$ may slightly overestimate dose to RBM and BSC in cranium spongiosa, it provides a significantly better estimate overall than $\mathrm{D}_{m}$, which consistently and significantly understimates dose to RBM and BSC. This observation is born out in the results in Table 7.3, where the average $\mathrm{D}_{w}$ is within $1.5 \%$ of average doses to RBM and BSC, while average $\mathrm{D}_{m}$ underestimates the average dose to RBM and BSC by at least $5 \%$. Note that this understimate is clinically-significant at the $5 \%$ level.

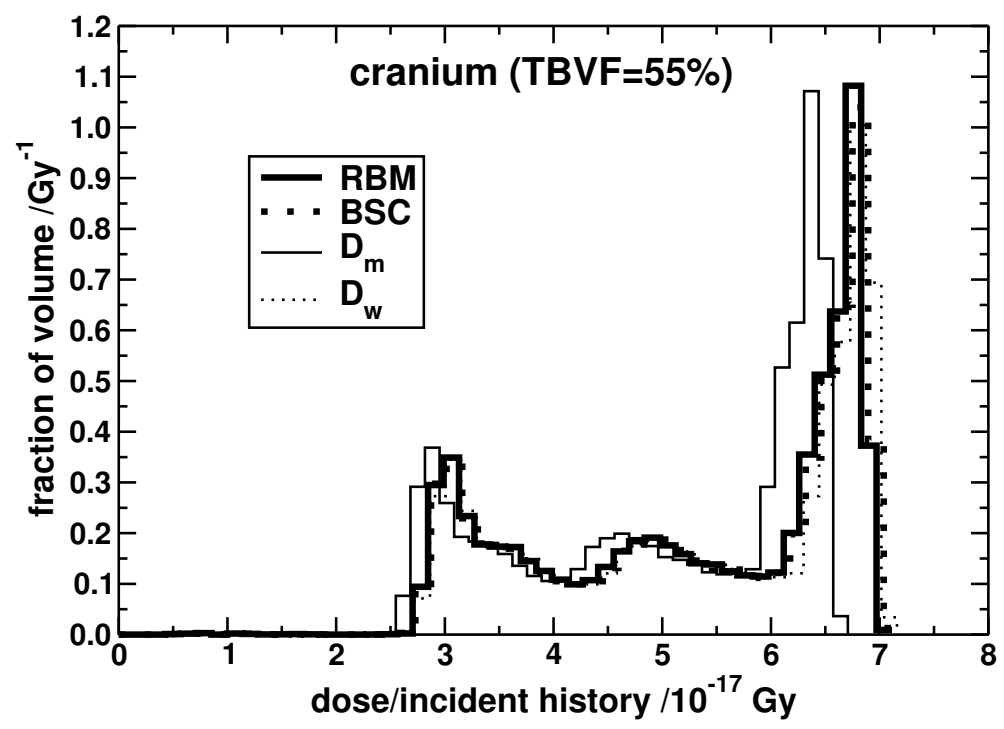

Figure 7.3: Differential DVH's for RBM, BSC, $\mathrm{D}_{m}$ and $\mathrm{D}_{w}$ calculated in the cranium spongiosa during the simulated head and neck irradition of FAX06. 


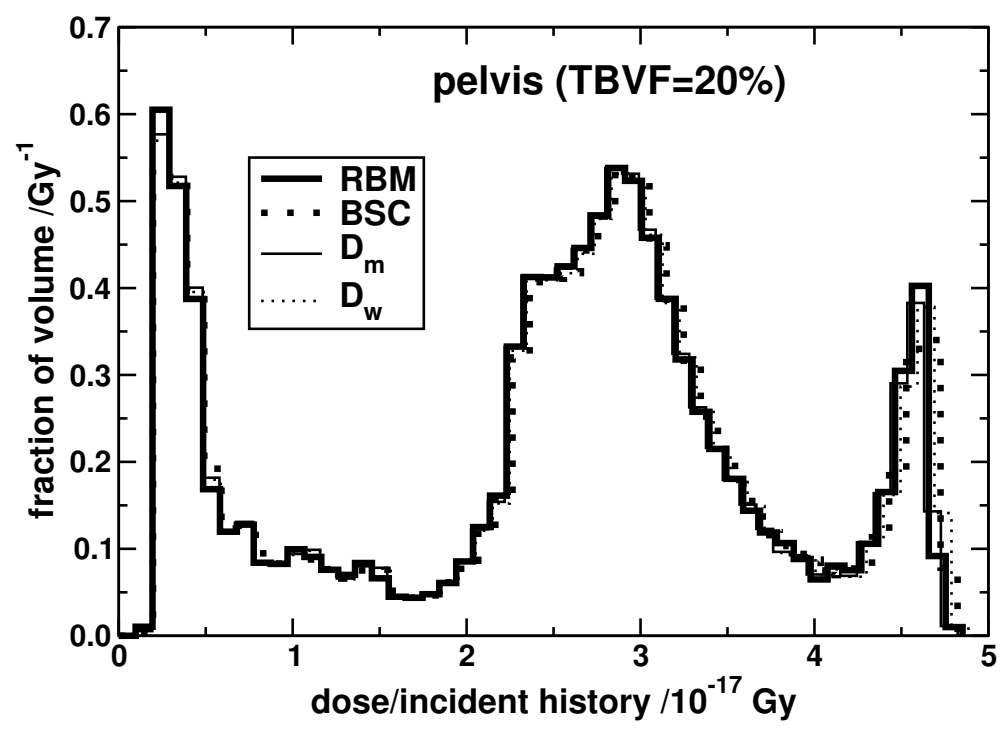

Figure 7.4: Differential DVH's for RBM, BSC, $\mathrm{D}_{m}$ and $\mathrm{D}_{w}$ in the pelvis spongiosa in the simulated pelvis irradiation of FAX06.

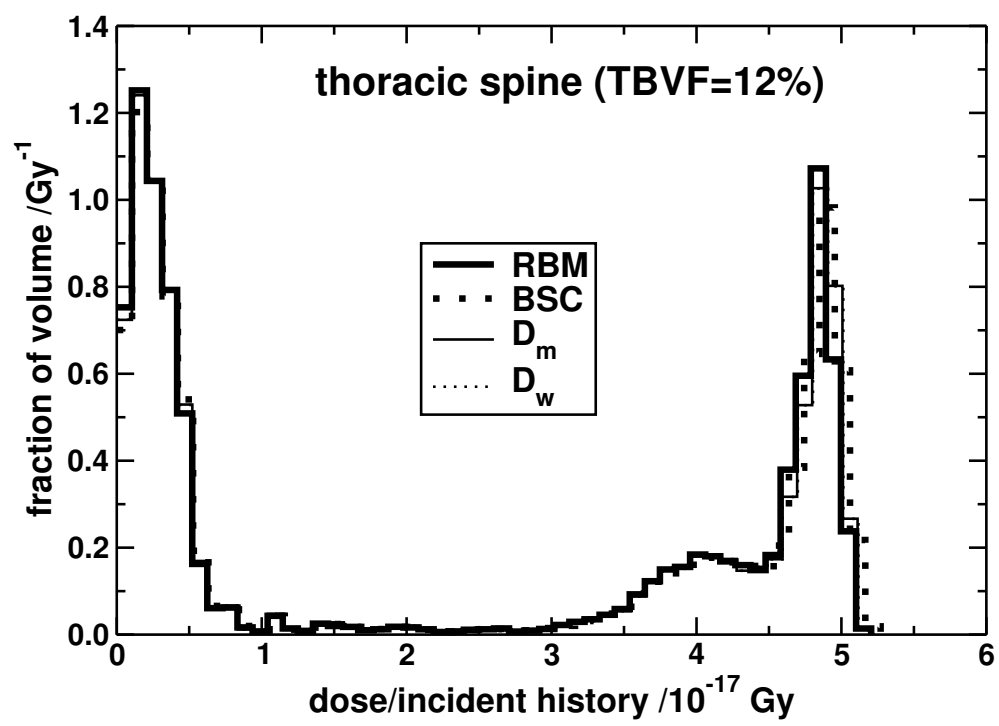

Figure 7.5: Differential DVH's for RBM, BSC, $\mathrm{D}_{m}$ and $\mathrm{D}_{w}$ in thoracic spine in the simulated chest irradiation of FAX06.

With decreasing TBVF, the difference between $\mathrm{D}_{w}$ and $\mathrm{D}_{m}$ becomes less significant. In pelvis spon- 


\begin{tabular}{|c|c|c|c|c|c|}
\hline location & TBVF (\%) & \multicolumn{4}{|c|}{ average dose/incident history $\left(10^{-17} \mathrm{~Gy}\right)$} \\
& & & RBM & BSC & homogeneous spongiosa \\
& & & & $\mathrm{D}_{m}$ & $\mathrm{D}_{w}$ \\
\hline cranium & 55 & 5.31 & 5.36 & 5.04 & 5.38 \\
pelvis & 20 & 2.48 & 2.51 & 2.47 & 2.50 \\
legs & 15 & 1.27 & 1.29 & 1.27 & 1.28 \\
thoracic spine & 12 & 2.38 & 2.41 & 2.40 & 2.40 \\
ribs & 10 & 1.16 & 1.18 & 1.17 & 1.17 \\
\hline
\end{tabular}

Table 7.3: Average RBM and BSC doses and average values of $\mathrm{D}_{m}$ and $\mathrm{D}_{w}$ at different spongiosa locations (and TBVF's) in FAX06. Locations are listed in order of decreasing TBVF.

giosa (TBVF=20\%), Figure 7.4 shows a difference between $\mathrm{D}_{w}$ and $\mathrm{D}_{m}$ DVH's that is statistically significant, but not likely to affect dose prescriptions in clinical practice. Table 7.3 shows that average $\mathrm{D}_{w}$ and $\mathrm{D}_{m}$ differ by $1.2 \%$. However, even at this level, it is clear from Figure 7.4 and Table 7.3 that $\mathrm{D}_{w}$ lies in between dose to RBM and BSC and provides a better overall estimate of dose to RBM and BSC (average values within $0.8 \%$ ), while $\mathrm{D}_{m}$ lies below dose to RBM and BSC and may provide a better estimate of the RBM dose (average values within $0.4 \%$ ) but is significantly lower than the dose to BSC (average value lower by $1.6 \%)$.

In the case of thoracic spine (TBVF=12\%), Figure 7.5 indicates the difference between $\mathrm{D}_{w}$ and $\mathrm{D}_{m}$ is not significant, and both estimate dose to BSC and RBM equally well, with average values within $0.8 \%$ of average dose to BSC and RBM.

Figure 7.6 shows the fractional differences between the average $\mathrm{D}_{w}$ and $\mathrm{D}_{m}$ and the average doses to RBM and BSC as a function of spongiosa TBVF. This figure is generated from the data in Table 7.3. From the figure, it is clear that, in cases where there is a significant difference between $\mathrm{D}_{m}$ and $\mathrm{D}_{w}, \mathrm{D}_{w}$ provides a better overall estimate of the dose to RBM and BSC considered together (or averaged).

\subsection{Conclusions}

The FAX06/MAX06 Monte Carlo codes are potentially powerful codes for performing dose calculations in typical patient structures, not least because they accurately model (based on $\mu \mathrm{CT}$ data) spongiosa structure and allow calculation of dose in the sensitive tissue (RBM and BSC) within the spongiosa. With some modification for this study, the FAX06/MAX06 EGSnrc user code is used to revisit the debate over whether dose to medium, $\mathrm{D}_{m}$, or dose to water, $\mathrm{D}_{w}$, should be used for radiotherapy treatment plans calculated using Monte Carlo methods. This debate ultimately centres around the dose to boney structures and the sensitive tissue contained therein because these are the only tissues in the body where the water-to-medium unrestricted stopping power ratio, $S_{m}^{w}$ differs sufficiently from unity that there is a potentially clinically-significant difference between $\mathrm{D}_{m}$ and $\mathrm{D}_{w}$.

If clinical significance is taken at the $5 \%$ level, then the results from this study show that, for the 6 MV treatment beam simulated here, a clinically-significant difference between $\mathrm{D}_{m}$ and $\mathrm{D}_{w}$ only exists in the cranium spongiosa, where $\mathrm{TBVF}=55 \%$. Here, the mean $\mathrm{D}_{m}$ and $\mathrm{D}_{w}$ calculated in homogeneous 


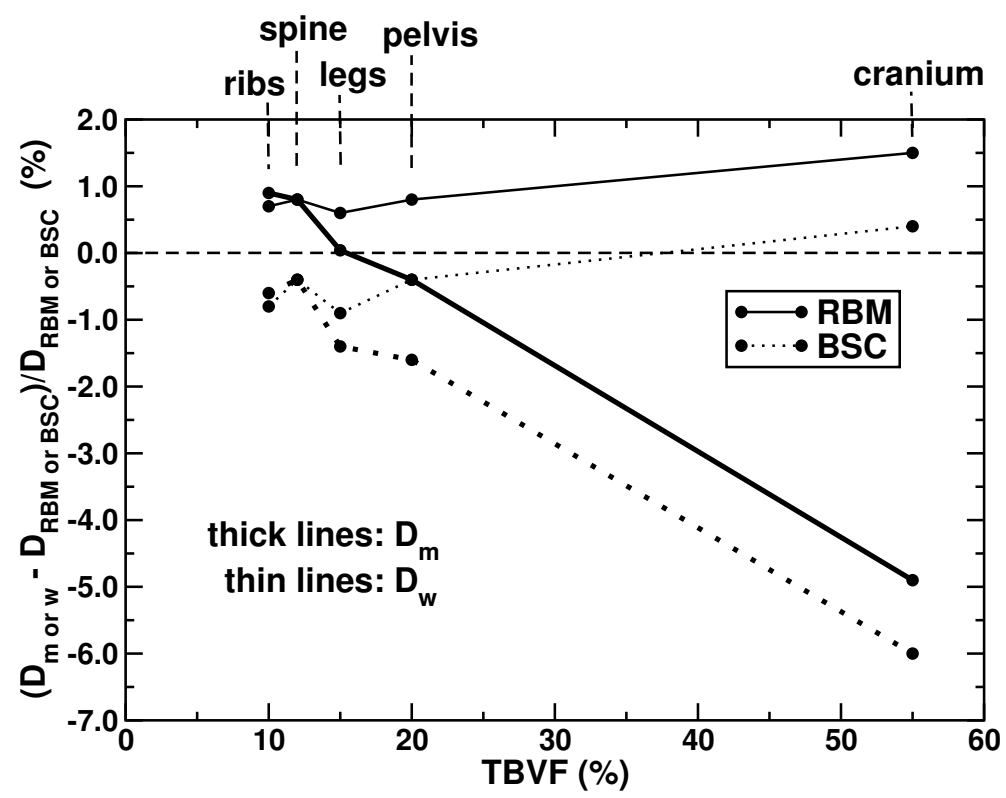

Figure 7.6: Fractional difference between the mean value of $\mathrm{D}_{m}$ and the mean doses to RBM and BSC (thick lines) and the mean value of $\mathrm{D}_{w}$ and mean doses to RBM and BSC (thin lines) as a function of TBVF.

spongiosa differ by more than $6 \%$. In the pelvis $(\mathrm{TBVF}=20 \%)$ the difference between $\mathrm{D}_{m}$ and $\mathrm{D}_{w}$ is significant ( 1.5\%) but not at the clinical level. For spongiosa with lower TBVF, the difference between $\mathrm{D}_{m}$ and $\mathrm{D}_{w}$ is not significant above the precision of these calculations (0.5\%)

In terms of which dose provides the better estimate of dose to RBM and BSC, Figure 7.6 makes it clear that, for spongiosa where the difference between $\mathrm{D}_{m}$ and $\mathrm{D}_{w}$ is significant, $\mathrm{D}_{w}$ provides the better overall estimate. Even in the pelvis, where $\mathrm{D}_{m}$ provides a better estimate of the dose to RBM ( $0.4 \%$ difference versus $0.8 \%$ for $\left.\mathrm{D}_{w}\right), \mathrm{D}_{w}$ still provides the better estimate of dose to RBM and BSC considered together (within $0.8 \%$ of both, while $\mathrm{D}_{m}$ is $1.6 \%$ lower than dose to BSC).

The results presented here can be generalized to other treatment beam energies since the water-tomedium stopping power ratio does not change in a significant way over the megavoltage energy range for all values of TBVF and the behaviour of dose calculated in RBM and BSC is not expected to change appreciably over this energy range either.

\subsection{Bibliography}

[1] I. Kawrakow, M. Fippel, and K. Friedrich. 3D Electron Dose Calculation using a Voxel based Monte Carlo Algorithm (VMC). Med. Phys., 23:445 - 457, 1996. 
[2] I. Kawrakow. Improved modeling of multiple scattering in the Voxel Monte Carlo model. Med. Phys., 24:505 - 517, 1997.

[3] M. Fippel. Fast Monte Carlo dose calculation for photon beams based on the VMC electron algorithm. Med. Phys., 26:1466 - 1475, 1999.

[4] I. Kawrakow and M. Fippel. Investigation of variance reduction techniques for Monte Carlo photon dose calculation using XVMC. Phys. Med. Biol., 45:2163 - 2184, 2000.

[5] I. Kawrakow. $\mathrm{VMC}^{++}$, electron and photon Monte Carlo calculations optimized for Radiation Treatment Planning. In A. Kling, F. Barao, M. Nakagawa, L. Távora, and P. Vaz, editors, Advanced Monte Carlo for Radiation Physics, Particle Transport Simulation and Applications: Proceedings of the Monte Carlo 2000 Meeting Lisbon, pages 229 - 236. Springer, Berlin, 2001.

[6] H. Neuenschwander, T. R. Mackie, and P. J. Reckwerdt. MMC- A high-performance Monte Carlo code for electron beam treatment planning. Phys. Med. Biol., 40:543 - 574, 1995.

[7] C.-M. Ma, J. S. Li, T. Pawlicki, S. B. Jiang, and J. Deng. MCDOSE - A Monte Carlo dose calculation tool for radiation therapy treatment planning. In W. Schlegel and T. Bortfeld, editors, The Use of Computers in Radiotherapy, XIIIth Int'l Conf., Heidelberg, pages 123 - 125. Springer-Verlag, Heidelberg, 2000.

[8] H. H. Liu and P. Keall. Point/Counterpoint: $\mathrm{D}_{m}$ rather than $\mathrm{D}_{w}$ should be used in Monte Carlo treatment planning. Med. Phys., 29:922 - 924, 2002.

[9] I. J. Chetty et al. Report of the AAPM Task Group No. 105: Issues associated with clinical implementation of Monte Carlo-based treatment planning. Med. Phys., 34:4818-4853, 2007.

[10] J. V. Siebers, P. J. Keall, A. E. Nahum, and R. Mohan. Converting absorbed dose to medium to absorbed dose to water for Monte Carlo based photon beam dose calculation. Phys. Med. Biol., 45: $983-995,2000$.

[11] N. Dogan, J. V. Siebers, and P. J. Keall. Clinical comparison of head and neck and prostate IMRT plans using absorbed dose to medium and absorbed dose to water. Phys. Med. Biol., 51:4967-4980, 2006.

[12] ICRP. Basic Anatomical and Physiological Data for use in Radiological Protection: The Skeleton. ICRP Report 70, ICRP, Washington D.C., 1995.

[13] ICRP. Limits for Intakes of Radionuclides by Workers. ICRP Report 30, ICRP, Washington D.C., 1979.

[14] R Kramer, H J Khoury, J W Vieira, and V J M Lima. MAX06 and FAX06: update of two adult human phantoms for radiation protection dosimetry. Phys. Med. Biol., 51:3331-3346, 2006. 
[15] I. Kawrakow and D. W. O. Rogers. The EGSnrc Code System: Monte Carlo simulation of electron and photon transport. Technical Report PIRS-701 (4th printing), National Research Council of Canada, Ottawa, Canada, 2003.

[16] R Kramer, H J Khoury, J W Vieira, and I Kawrakow. Skeletal dosimetry in the MAX06 and the FAX06 phantoms for external exposure to photons based on vertebral 3D-microCT images. Phys. Med. Biol., 51:6265-6289, 2006.

[17] J W Vieira R Kramer, H J Khoury and I Kawrakow. Skeletal dosimetry for external exposure to photons based on $\mu \mathrm{CT}$ images of spongiosa from different bone sites. Phys. Med. Biol., 52:66976716, 2007. http://stacks.iop.org/0031-9155/52/6697.

[18] B. R. B. Walters, G. X. Ding, R. Kramer, and I. Kawrakow. Skeletal dosimetry in cone beam computed tomography. Med. Phys., 36:2915 - 2922, 2009.

[19] ICRP. Basic Anatomical and Physiological Data for Use in Radiological Protection: Reference Values. Annals of the ICRP 89, ICRP, Washington D.C., 2002.

[20] ICRU. Tissue Substitutes in Radiation Dosimetry and Measurements. ICRU Report 44, ICRU, Washington D.C., 1989.

[21] D. W. O. Rogers, B. Walters, and I. Kawrakow. BEAMnrc Users Manual. NRC Report PIRS 509(a)revI, 2005.

[22] B. R. B. Walters, I. Kawrakow, and D. W. O. Rogers. DOSXYZnrc Users Manual. NRC Report PIRS $794(\operatorname{rev} B), 2005$.

[23] D. W. O. Rogers, I. Kawrakow, J. P. Seuntjens, B. R. B. Walters, and E. Mainegra-Hing. NRC User Codes for EGSnrc. Technical Report PIRS-702(RevB), National Research Council of Canada, Ottawa, Canada, 2003.

[24] I. Kawrakow. EGSnrc C++ class library. Technical Report PIRS-898, National Research Council of Canada, Ottawa, Canada, 2005.

[25] E. Tonkopi, M. McEwen, B.R.B. Walters, and I. Kawrakow. Influence of ion chamber response on in-air profile measurements in megavoltage photon beams. Med. Phys., 32:2918-2927, 2005.

[26] I. Kawrakow and B. R. B. Walters. Efficient photon beam dose calculations using DOSXYZnrc with BEAMnrc. Med. Phys., 33:3046 - 3056, 2006.

[27] Iwan Kawrakow, D. W. O Rogers, and B.R.B. Walters. Large efficiency improvements in BEAMnrc using directional bremsstrahlung splitting. Med. Phys., 31:2883 - 2898, 2004. 

Discussion and Summary 



\subsection{Discussion}

Research described in this thesis focuses on aspects of making Monte Carlo (MC) dose calculations in photon beams more efficient with a view to facilitating the use of MC in the lab and clinic. The final two papers exemplify the application of MC dose calculations to yield information not available analytically, in the first case to quantify elevated dose to radiosensitive bone surface cells (BSC) in bone marrow during image-guided ratiotherapy (IGRT), and in the second to relate dose to bulk medium (bone), $\mathrm{D}_{m}$, and dose to water-in-medium, $\mathrm{D}_{w}$, to the actual dose received by BSC and red bone marrow (RBM) during treatment.

\subsubsection{Statistical considerations and the argument for eliminating phase space files}

Given the stochastic nature of MC calculations, an essential element to any application is a robust method for estimating uncertainties in simulated quantites. As described in chapter II[1], a history by history method for estimating uncertainties has been implemented in all EGSnrc user codes. This replaced the former method, based on statistical batches which, while relatively robust, was subject to large variations and, as implemented, disregarded correlations between incident particles when using phase space data from an upstream simulation as a source. The latter limitation was poised to become a significant issue with the introduction of directional bremsstrahlung splitting (DBS) to increase the efficiency of photon beam simulations. Optimum efficiency with DBS requires the use of large splitting numbers (see chapter IV[2]) and, hence, results in many correlated particles in the phase space data collected at the bottom of the simulated treatment head. One could argue that, owing to the stability and robustness of the history by history method, its introduction into EGSnrc applications has been a necessary step towards the development of the second-generation variance reduction techniques, such as directional bremsstrahlung splitting (DBS).

Variants of the history by history method for uncertainty estimation had already been used in MCNP[3], PENELOPE[4] and Geant4[5] codes before it was implemented in EGSnrc. However, the widespread adoption of EGSnrc by the research community has ensured thorough benchmarking and documentation of the technique.

This statistical technique is not without its limitations, however. Chapter II makes it clear that when particle phase space data are used as a source, the uncertainty estimate is invalid if correlations between incident particles are lost. To this end, a negative energy marker was introduced into the standard BEAMnrc-format phase space file to indicate the beginning of data scored by a new primary history (statistically independent event). This method of separating statistically independent data has also been adopted by the IAEA[6] in the format standard for their online phase space data library.

Nevertheless, it is common that there are not enough particles in the phase space data to obtain the desired level of uncertainty in a downstream calculation. In this case, the user has recourse to reusing, or "recycling," particle data. While particle recycling can result in the expected decrease in uncertainty by a factor of $1 / \sqrt{\text { no. of histories }}$ at depth within a phantom, in general, the user will run up against 
the statistical limitations of the phase space data itself (i.e., the "latent variance" of the data). In many production runs, even with particle recycling, the user finds it difficult to avoid running out of phase space data, requiring that they restart from the first particle scored ("rewind" the phase space data). This entails an accompanying loss in information about correlations and renders uncertainty estimates invalid.

This raises the question of whether or not it is more convenient, both from a data logistics and statistics point of view, to use a BEAMnrc treatment head simulation compiled as a shared library as a source, especially since most options available with phase space sources in EGSnrc applications are also available with shared library sources.

A preference for simulation sources is further motivated by the results from chapter IV, which show that, provided the treatment head simulation is optimized (i.e., using the the most efficient DBS settings) then it may be possible to decrease the fraction of CPU time spent generating source particles to an acceptable minimum. In a more detailed study of dose calculation efficiency, Kawrakow and Walters[7] show that, provided that photon splitting/interaction forcing is used to maximize the efficiency of the in-phantom portion of the calculation, the maximum efficiency using an optimized treatment head simulation as a source ( $6 \mathrm{MV}$ beam, $10 \times 10 \mathrm{~cm}^{2}$ field) is only $5 \%$ less than that using a phase space file collected above the field-defining jaws. They note that among the factors contributing to the efficiency of the full treatment head simulation is the fact that the DBS parameters can be specifically tailored to the treatment field being used.

In addition, simulation sources open up possibilities for additional data to be conveniently passed between the the source and the dose calculation. For example, a significant development in BEAMnrc/DOSXYZnrc has been the synchronized sources implemented by Popescu and Lobo[8]. These allow for synchronization between the leaf positions of a multi-leaf collimator (MLC) in the treatment head and the incident angle/position of the source relative to the phantom, thus allowing the user to simulate, in a single run, an entire treatment protocol. While it is possible to use phase space data in these simulations, this requires scoring of an additional monitor unit parameter, resulting in an increasing in phase space file size.

Despite the clear advantages of using full treatment head simulations, compiled as shared libraries, as sources, many researchers continue to use phase space files[9-13]. There are several reasons for this. First of all, accurate simulation of accelerator geometry details usually requires access to proprietary data. Second, in general, the portion of the accelerator upstream of the field defining jaws or MLC remains fixed, meaning that phase space data need be collected only once above the jaws or the MLC, with simulation of specific treatment beams requiring a relatively short second stage simulation through the field defining aperture. The relative speed and simplicity of this second stage simulation, coupled with a decreasing cost of data storage that makes long-term storage of large phase space files feasible, reduces the incentive to switch to simulation sources. Finally, there are now a number of online libraries, provided by researchers and manufacturers, that offer free access to accelerator phase space data. In 2005, the IAEA initiated one of the most coordinated of these efforts, encouraging researchers to upload phase space data in a standardized file format[6] from any simulated external radiotherapy beam to a pub- 
licly accessible website. Shortly after this database was established, developers implemented the ability to read/write IAEA-format phase space data many of the major general purpose MC codes. This data base remains a particularly compelling opportunity to continually update the phase space data for more popular treatment heads as more accurate simulations become available. As of early 2017, however, the latest contributions to the IAEA library dated from 2011. One reason for this lag, apart from reluctance to provide proprietary accelerator information, is the fact that some manufacturers are now providing their own phase space data. For example, Varian now makes phase space files for their popular Truebeam accelerators available for free with registration on their company website[14].

In terms of commercial treatment planning applications, Kawrakow and Walters[7] point out an important limitation of shared library sources: MC in commercial treatment planning systems (TPSs) often uses highly optimized codes, such as VMC++[15] and XVMC[16], which are sufficiently fast to render the production of source particles by a full treatment head simulation, however efficient, a computational bottleneck. In fact, even the reading of phase space data from a file may be a time limiting factor, and many such treatment planning systems have recourse to simplified source models, or parameterizations, of the particular beams used. Hasenblag et al demonstrated that it is possible to carry out an entire linace simulation using $\mathrm{VMC}++[17]$. However, translation of a linac geometry into a form readable by a voxelbased code is not straightforward. Moreover, many of the benchmarked, high-speed dose calculation algorithms are now proprietary, putting them beyond the reach of most researchers.

More about the use of MC in commercial TPSs will be discussed in section 8.1.4 below.

\subsubsection{Acceptance of a split reality}

While directional bremsstrahlung splitting (DBS-see chapter IV) was originally developed to increase the efficiency of megavoltage (MV) photon beam simulations, it has also been shown to result in large efficiency improvements in kilovoltage $(\mathrm{kV}) \mathrm{X}$-ray tube simulations. Table 8.1 summarizes photon fluence efficiency results for DBS applied to simulated X-ray tubes, from Mainegra-Hing and Kawrakow[18]. These are shown with one set of results for DBS applied to a $6 \mathrm{MV}$ beam, taken from chapter IV in this thesis, and one set of results for directional source biasing (DSB) applied to a Co-60 beam, taken from chapter $\mathrm{V}$ [19]. Note that, although there is a large discrepancy in field size between the $\mathrm{kV}$ ( $\mathrm{r}=5 \mathrm{~mm}$ field) and MV $\left(10 \times 10 \mathrm{~cm}^{2}\right.$ field $)$ calculations, fluence efficiency in the MV calculations is analyzed by dividing the field into $1 \times 1 \mathrm{~cm}^{2}$ scoring zones, making all calculations roughly equivalent in terms of the effects of correlations between scored particles. Mainegra-Hing and Kawrakow realize factors of $1.6 \times 10^{6}(50 \mathrm{kVp})$ and $2.9 \times 10^{5}(160 \mathrm{kVp})$ improvement in photon fluence efficiency, much greater than the factor of $500 \mathrm{in}$ crease in fluence efficiency when DBS is used in a 6 MV linac. Mainegra-Hing and Kawrakow attribute the greater efficiency improvement in $\mathrm{kV}$ beams to the fact that photons resulting from bremsstrahlung events radiate over a solid angle closer to $4 \pi$ (with the solid angle increasing with decreasing tube potential), resulting in DBS immediately eliminating a greater fraction of photons directed outside the field of interest. Note, also that while the optimum bremsstrahlung splitting numbers for MV beams are on the order of $10^{3}$, efficiency of DBS for $\mathrm{kV} \mathrm{X}$-rays is maximized at bremsstrahlung splitting numbers on 
the order of $10^{6}$. Moreover, Mainegra-Hing and Kawrakow show that the improvement in X-ray fluence efficiency using DBS is a factor of 60 over that achieved using optimized uniform bremsstrahlung splitting (UBS), the variance reduction method of choice for X-ray tube simulation using BEAMnrc up to the time of their study[20]. This improvement over UBS is significantly higher than the factor of 20 for optimized DBS at $6 \mathrm{MV}$.

Table 8.1: Summary of maximum photon fluence efficiency gains relative to no splitting when directional bremsstrahlung splitting (DBS) is applied to $50,160 \mathrm{kVp} \mathrm{X}$-ray tube simulations and a $6 \mathrm{MV}$ linac simulation. Results for uniform bremsstrahlung splitting (UBS) are shown for the $160 \mathrm{kVp}$ and $6 \mathrm{MV}$ cases. In addition, results for directional source biasing (DSB) are shown for a Co-60 beam. In all cases, electron splitting is turned off. Results are taken from Mainegra-Hing and Kawrakow[18] $\left.{ }^{*}\right)$, chapter IV of this thesis $(\dagger)$, and chapter $V$ of this thesis ( $\ddagger)$.

\begin{tabular}{|c|c|l|l|}
\hline beam energy (field size) & VR method & $\begin{array}{l}\text { max. relative photon } \\
\text { fluence eff. }\end{array}$ & splitting no. \\
\hline \hline $50 \mathrm{kVp}(\mathrm{r}=5 \mathrm{~mm})^{*}$ & DBS & $1.6 \times 10^{6}$ & $5.5 \times 10^{6}$ \\
\hline \multirow{2}{*}{$160 \mathrm{kVp}(\mathrm{r}=5 \mathrm{~mm})^{*}$} & DBS & $2.9 \times 10^{5}$ & $1 \times 10^{6}$ \\
& UBS & 4,800 & $7.2 \times 10^{4}$ \\
\hline \multirow{2}{*}{$6 \mathrm{MVp}\left(10 \times 10 \mathrm{~cm}^{2}\right)^{\dagger}$} & DBS & 500 & 2,500 \\
& UBS & 25 & 750 \\
\hline $\mathrm{Co}-60\left(10 \times 10 \mathrm{~cm}^{2}\right)^{\ddagger}$ & DSB & 1,800 & $2 \times 10^{4}$ \\
\hline
\end{tabular}

The large optimum splitting numbers found by Mainegra-Hing and Kawrakow reflect the inefficiency of MC simulation of near-isotropic radiative events. This observation also applies to the directional source biasing (DSB) algorithm, described in chapter V of this thesis. When DSB is used in a simulated Co-60 beam, the maximum photon fluence efficiency gain (a factor of 1,800 ) is achieved with an optimum splitting number of $2 \times 10^{4}$. Note, though, that even with this large splitting number, once photons not directed into the field are eliminated and circular symmetry above the jaws is taken into account, only $\sim 3$ split photons per primary history are actually simulated. This implies that there is a plethora of relevant information in relatively few statistically independent events, and, while splitting of radiative events is essential for efficient photon beam simulation, future improvements may hinge on increasing the efficiencies of the splitting algorithms themselves.

\subsubsection{The $\mathbf{D}_{m}$ vs $\mathbf{D}_{w}$ debate}

The dose calculations in voxellized human phantoms described in chapters VI[21] and VII[22] offer an exciting glimpse of the potential for MC simulations to improve our understanding of the radiobiological effects (RBE) of radiation. The research in chapter VII indicates that, for megavoltage radiotherapy beams, dose to water-in-medium, $\mathrm{D}_{w}$, which has traditionally been reported by treatment planning systems, provides a better predictor of the RBE in sensitive tissue (red bone marrow and bone surface cells) within bone spongiosa than does dose to medium, $\mathrm{D}_{m}$, the quantity calculated by default in all MC codes. To put this conclusion in perspective, however, the differences between $\mathrm{D}_{m}$ and $\mathrm{D}_{w}$ in red bone marrow and bone surface cells are only clinically significant at the $5 \%$ level when the trabecular volume frac- 
tion (TBVF) in the spongiosa is greater than $\sim 40 \%$. This is typically only the case in the cranium and mandibles.

The debate over whether $\mathrm{D}_{w}$ or $\mathrm{D}_{m}$ is a better indication of RBE has now been going on for over a decade and a half (since the incorporation of MC calculation in TPSs was anticipated), with compelling arguments on both sides. In megavoltage treatment beams, differences are only significant in bone[2325]. This is due to the fact that the $\mathrm{D}_{m}$ is converted to $\mathrm{D}_{w}$ using the ratio of mass collision stopping power in water to that in the medium, $\bar{S}_{m}^{w}$ (where $\bar{S}$ indicates that stopping powers are average over the primary electron fluence spectrum) which, for MV beams, is close to unity for all tissues except bone. An important underlying assumption in this conversion method is that the volume of interest behaves like a Bragg-Gray (BG) cavity. That is: 1) the cavity dimension is small compared to the average range of secondary charged particles, 2) the cavity does not perturb secondary charged particle fluence, and 3) the dose in the cavity is due to charged particles crossing the cavity, not created in it.

The situation becomes more complicated at photon energies in the kilovoltage range. Here, one cannot assume that a volume of interest (cavity) will fulfill BG conditions. Conversion of $\mathrm{D}_{m}$ to $\mathrm{D}_{w}$ must now account for a significant fraction of charged particles generated within the volume. In the extreme case, for a sufficiently low energy/large cavity, conditions of charged particle equilibrium (CPE) prevail, and $\mathrm{D}_{m}$ is converted to $\mathrm{D}_{w}$ using the ratio of mass-energy transfer coefficients, $\left[\frac{\overline{\mu_{e n}}}{\rho}\right]_{m}^{w}$, (where $\frac{\overline{\mu_{e n}}}{\rho}$ indicates mass energy transfer coefficiencts are average over the primary photon energy fluence spectrum). In other words, one can assume that dose is equal to kerma.

The method by which $\mathrm{D}_{m}$ is converted to $\mathrm{D}_{w}$ is of particular importance in brachytherapy, where MC techniques have gained some foothold in commercial TPSs and are also extensively used for commissioning. The current brachytherapy dosimetry protocol, based on a revised version of the AAPM TG-43 formalism [26, 27], implicitly assumes CPE conditions exist. Moreover, the prevailing assumption is that a volume of water-in-medium is subject to the same photon energy fluence as the medium itself. This is not, in general, true, especially when there is a large difference in Z-value between water and the medium in question (e.g., bone). Gimenez-Alventosa et al[28], thus, propose the additional use of a photon energy fluence correction factor. The situation is further complicated by the rapid change in $\left[\frac{\overline{\mu_{e n}}}{\rho}\right]_{m}^{w}$ with energy below $200 \mathrm{keV}$, requiring that the ratio be evaluated over the entire local photon spectrum instead of just taking an average value[29].

Another application in which the question of the method used to convert $\mathrm{D}_{m}$ to $\mathrm{D}_{w}$ is relevant is small animal radiotherapy. Recent interest in small animal radiotherapy-where beam energies are in the $\mathrm{kV}$ range and, for dose calculations, phantom voxels may have sub-mm dimensions-has grown due to its potential for preclinical evaluation of advanced radiotherapy techniques from both a technical and RBE perspective[30, 31].

Tedgren and Carlsson[32] have proposed a method for estimating $\mathrm{D}_{w}$ for brachytherapy dose calculations in intermediate-size volumes based on the cavity theory of Burlin[33]. Burlin's theory states that

$$
\frac{D_{w}}{D_{m}}=d \bar{S}_{m}^{w}+(1-d)\left[\frac{\overline{\mu_{e n}}}{\rho}\right]_{m}^{w}
$$


The parameter, $d$, is the fractional contribution to cavity dose from electrons generated outside the cavity (i.e., in the cavity wall), while 1- $d$ is the fractional contribution to dose from electrons generated within the cavity. The value of $d$ is related to the attenuation of wall electrons as they cross the cavity and the buildup of electrons generated in the cavity. In Burlin's original formulation, these processes are assumed to be governed by the same exponential coefficient, and $d$ is given by:

$$
d=\frac{\left(1-e^{-\beta g}\right)}{\beta g}
$$

where $\beta$ is the mass attenuation coefficient of primary electrons and $g$ is the mean chord length of the cavity. For a convex cavity, $g=\frac{4 V}{S}$, where $V$ is the cavity volume and $S$ is its surface area. Thus, Burlin's theory is a weighted sum of contributions from charged particles in a cavity obeying BG conditions and photons under conditions of CPE.

Several methods for determining $d$ have been proposed. These include the use of MC to track the fraction of cavity dose due to electrons originating within the cavity[34] and several different methods for calculating $\beta$ in Equation 8.2[35-37]. In a recent study of $\mathrm{D}_{w}$ in $\mu \mathrm{m}$-size spherical cavities, intended to simulate cell and cell nuclei, Oliver and Thomson[38] found that agreement between Burlin's theory and direct MC-computed values of $\mathrm{D}_{w}$ was best when Equation 8.2 was used with the $\beta$ calculated using the method of Janssens et al[36]:

$$
1-e^{-\beta R}=0.96
$$

where $R$ is the range in water of electrons with the maximum kinetic energy.

The value of $g$ used in Equation 8.2 will depend on the dimensions of the relevant cavity. Tedgren and Carlsson[32] interpret the cavity as being of typical nuclear $(\sim 10 \mu \mathrm{m})$ or cellular $(\sim 100 \mu \mathrm{m})$ dimensions, while Oliver and Thomson [38] consider cavity dimensions only in the $\leq 10 \mu \mathrm{m}$ range. In the DOSXYZnrc calculation described below, however, the cavity is assumed to have dimensions equal to those of the rectilinear voxels comprising the phantom. There is some rationale for this, as the voxel dimensions define the maximum resolution of the dose calculation, regardless of the scale at which the physical processes contributing to RBE operate.

As an illustration of how Burlin's theory might apply to small animal treatment planning, Figure 8.1 depicts dose isolines (and the equivalent colour wash) resulting from a DOSXYZnrc calculation in a CTimage-based mouse phantom $(0.25 \mathrm{~mm} \times 0.25 \mathrm{~mm} \times 1 \mathrm{~mm}$ voxels $)$ exposed to a nominal $225 \mathrm{kVp}$ X-ray beam $(1 \mathrm{~cm} \times 1 \mathrm{~cm}$ field). The photon energy spectrum is calculated[39] based on specifications for an X-Rad 225Cx small animal irradiator (Precision X-ray Inc., North Branford, CT, USA). The spectrum has a minimum energy of $22.5 \mathrm{keV}$, a maximum energy of $225 \mathrm{keV}$, an average energy of $85.7 \mathrm{keV}$, with characteristic peaks at $59.6 \mathrm{keV}$ (relative intensity $\equiv$ ) and $67.5 \mathrm{keV}$ (relative intensity=0.43). For a beam incident from the right, the figure shows (a) dose to medium $\mathrm{D}_{m}$; (b) dose to water-in-medium, $\mathrm{D}_{w}$, assuming voxels are intermediate-size cavities and using Equation 8.1) with the value of $d(0.63)$ calculated using the mean voxel chord length $(0.22 \mathrm{~mm})$ and the range of maximum kinetic energy $(224.5 \mathrm{keV})$ electrons in water $(\sim 0.72 \mathrm{~mm})$ in Equations 8.2 and 8.3 ; (c) $\mathrm{D}_{w}$ assuming $\mathrm{BG}$ conditions in the voxels 
(Equation 8.1 with $d=1$ ), and (d) $\mathrm{D}_{w}$ assuming CPE prevails in the voxels (Equation 8.1 with $d=0$ ). Note that dose is shown as a percent of the average of the maximum 20 values of $D_{m}$ evaluated over the entire phantom, hereinafter referred to as $\mathrm{D}_{\operatorname{m}, \max 20}$. Soft tissue (lung, adipose tissue and muscle) assignment in the phantom used in this calculation should be taken as highly approximate, since the original CT conversion function associated with this image differentiated soft tissue only on the basis of lung and muscle. The three-soft-tissue conversion function is used here for illustrative/investigative purposes.

Figure 8.2 shows the water-to-medium ratios of (a) mass collision stopping powers, $\bar{S}_{m}^{w}$, and (b) mass energy transfer coefficients, $\left[\frac{\overline{\mu_{e n}}}{\rho}\right]_{m}^{w}$ calculated on a voxel-by-voxel basis and used to calculate $\mathrm{D}_{w}$ in Figure 8.1. Stopping power data are from ICRU37[40]. Electron primary fluence spectrum-averaged stopping power ratios are calculated according to the Spencer-Attix formulation[41] using a method similar to that used in the EGSnrc application, SPRRZnrc[42], which does not require a priori calculation of the electron fluence spectrum in each voxel. Calculation of the photon energy fluence-averaged values of $\frac{\mu_{e n}}{\rho}$ makes use of precalculated tables of mass-energy absorption coefficient as a function of energy (on a linear scale) for each medium. These tables are generated using the EGSnrc application, g, which determines $\frac{\mu_{e n}}{\rho}$ from collision kerma.

For comparison with Figure 8.2, Table 8.2 shows values of $\bar{S}_{m}^{w}$ calculated at $2 \mathrm{~mm}$ depth in medium using SPRRZnrc with the $225 \mathrm{kVp}$ X-ray spectrum evaluated after passing through $1.375 \mathrm{~cm}$ of water (i.e., approximately half the cross sectional thickness of the mouse). Table 8.2 also shows $\left[\frac{\overline{\mu_{e n}}}{\rho}\right]_{m}^{w}$ values analyzed at the mean energy of the X-ray spectrum after passing through $1.375 \mathrm{~cm}$ of water $(85.8 \mathrm{keV})$. Note, evaluating $\left[\frac{\overline{\mu_{e n}}}{\rho}\right]_{m}^{w}$ at a single, mean energy avoids the necessity of averaging $\frac{\mu_{e n}}{\rho}$ values over the photon energy fluence spectrum, as was done for Figure 8.2, but potentially introduces inaccuracies because of the large variation in $\frac{\mu_{e n}}{\rho}$ with photon energy at low $\mathrm{keV}$.

Note that each isoline in Figures 8.1 and 8.2 encompasses a region where the quantity of interest (dose, stopping power ratio or ratio of mass energy transfer coefficients) falls between the value at the isoline and the next isoline on the scale. Similarly, for the colour wash, a single colour is used for all quantities falling between the value associated with that colour and the next value on the scale. Therefore, it makes sense to refer to ranges of a given quantity when analyzing these images.

Dose to medium results, Figure 8.1(a), show the expected local maxima in bone (pelvis, sternum, spine) and steep fall-off to values $<20 \%$ of $\mathrm{D}_{\operatorname{m} \text {, max } 20}$ beyond the edges of the field. Overall, dose decreases with increasing distance from the beam entry surface, with doses in the pelvis (bone closest to beam entry), sternum and spine (bone farthest from beam entry) reaching maxima in the range $100-105 \%$, 95 $100 \%$ and $70-80 \%$ of $D_{m, \max 20}$, respectively. Note that, within a given bone region, the higest doses occur closest to the beam entry side. Also, note that dose to lungs is $\sim 20 \%$ lower than that to surrounding tissue, decreasing from $25-30 \%$ to $20-25 \%$ of $\mathrm{D}_{m \text {, } \max 20}$. On closer inspection, it was found that, owing to the approximate nature of the conversion function used to create the DOSXYZnrc phantom, large portions of the lung were misregistered as adipose tissue. 


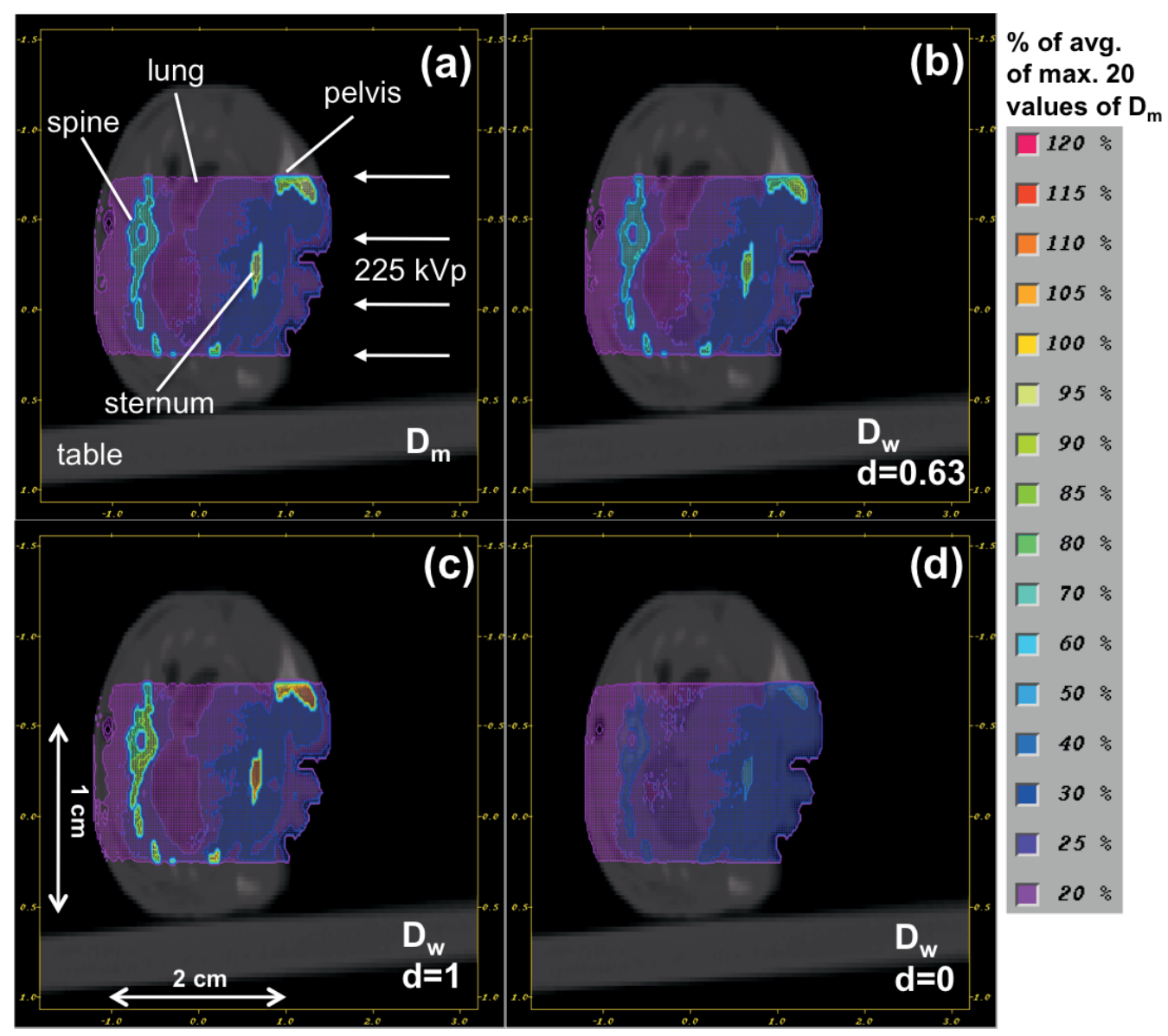

Figure 8.1: Dose isolines and colour wash resulting from a DOSXYZnrc simulation of a $225 \mathrm{kVp}$ beam $(1 \mathrm{~cm} \times 1 \mathrm{~cm}$ field $)$ from a small animal irradiator incident on a CT-image-based mouse phantom $(0.25$ $\mathrm{mm} \times 0.25 \mathrm{~mm} \times 1 \mathrm{~mm}$ voxels) from the right. The figure shows (a) dose to medium, $\mathrm{D}_{m}$; (b) dose to water-in-medium, $\mathrm{D}_{w}$, calculated using Burlin's theory for intermediate-size cavities (Equation 8.1) with the value of $d$ calculated using the mean voxel chord length $(0.22 \mathrm{~mm})$ and range in water of electrons with the maximum kinetic energy $\left(224.5 \mathrm{keV}\right.$, range $0.72 \mathrm{~mm}$ ) in Equations 8.2 and 8.3 ; (c) $\mathrm{D}_{w}$ calculated assuming voxels obey Bragg-Gray (BG) conditions (Equation 8.1 with $d=1$ ); and (d) $\mathrm{D}_{w}$ calculated assuming charged particle equilibrium (CPE) prevails in the voxels (Equation 8.1 with $d=0$ ). Doses are shown as a percentage of the average of the maximum 20 values of $\mathrm{D}_{m}, \mathrm{D}_{m, \max 20}$. An additional isoline at $25 \%$ has been added for resolution at lower doses. Media comprising the phantom are: air, lung, adipose, muscle and bone. Media composition and stopping powers are taken from ICRU37[40]. Voxel dimensions are $0.25 \mathrm{~mm} \times 0.25 \mathrm{~mm} \times 1 \mathrm{~mm}$. Calculations are run with $\mathrm{ECUT}=\mathrm{AE}=1 \mathrm{keV}$ and $\mathrm{PCUT}=\mathrm{AP}=1$ $\mathrm{keV}$. Note the expanded vertical scale for improved visualization. CT image courtesy of Ana Vaniqui. 

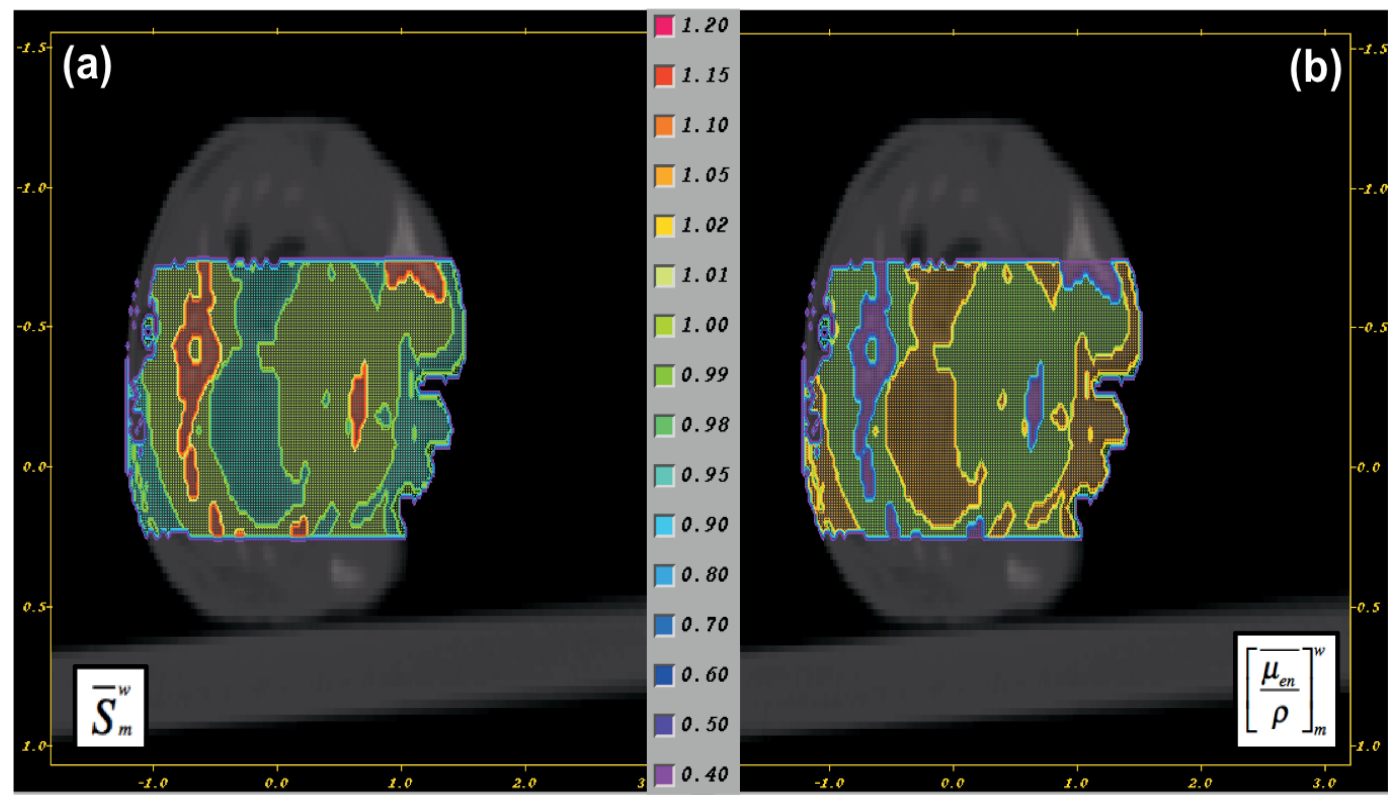

Figure 8.2: Voxel-by-voxel ratios of water-to-medium (a) mass collision stopping powers and (b) mass energy transfer coefficients for the $225 \mathrm{kVp}$ mouse treatment scheme shown in Figure 8.2. The colour scale for both panels is shown in the centre. Stopping powers are taken from ICRU37[40], and mass energy transfer coefficients are calculated using the EGSnrc application, g. Ratios are not shown in regions where $\mathrm{D}_{m}<20 \%$ of $\mathrm{D}_{m, \max 20}$.

Table 8.2: Mass collision stopping power ratios, $\bar{S}_{m}^{w}$, calculated at $2 \mathrm{~mm}$ depth in medium using the $225 \mathrm{kVp} \mathrm{X}$-ray spectrum after having passed through $1.375 \mathrm{~cm}$ of water, and water-to-medium ratios of mass energy transfer coefficients, $\left[\frac{\overline{\mu_{e n}}}{\rho}\right]_{m}^{w}$, at the mean energy of the X-ray spectrum after having passed through $1.375 \mathrm{~cm}$ of water $(85.8 \mathrm{keV})$. Water-to-medium stopping power ratios are calculated in centralaxis voxels (voxel thickness $=0.25 \mathrm{~mm}$, radius $=1 \mathrm{~mm}$ ) for a circular beam of radius $0.5 \mathrm{~cm}$ incident on one end of a cylindrical phantom (radius $=25 \mathrm{~cm}$ ) comprised of the medium using the EGSnrc application, SPRRZnrc. Values of $\frac{\mu_{e n}}{\rho}$ are calculated using the EGSnrc application, g, with a pencil beam incident on an infinite slab of the medium. Statistical uncertainty on all values is less than $0.05 \%$.

\begin{tabular}{|c|c|c|}
\hline medium & $\bar{S}_{m}^{W}$ & {$\left[\frac{\overline{\mu_{e n}}}{\rho}\right]_{m}^{W}$} \\
\hline air & 1.552 & 1.085 \\
lung & 1.009 & 0.993 \\
adipose & 0.963 & 1.080 \\
muscle & 1.008 & 0.996 \\
bone & 1.197 & 0.427 \\
\hline
\end{tabular}

If the intermediate size of the voxels is accounted for (i.e., using Equation 8.1 with $d$ determined by Equations 8.2 and 8.3) when calculating $\mathrm{D}_{w}$, then Figure 8.1(b) shows that, for this particular case, $\mathrm{D}_{w}$ 
in soft tissue is similar to $\mathrm{D}_{m}$, while $\mathrm{D}_{w}$ in bone is $5-10 \%$ lower, with maxima of $90-95 \%$ of $\mathrm{D}_{m, \max 20}$ in the pelvis and sternum and large regions of the spine where $\mathrm{D}_{m}$ is $70-80 \%$ of $\mathrm{D}_{m, \max 20}$ having $\mathrm{D}_{w}$ values of $60-70 \%$ of $\mathrm{D}_{\operatorname{m}, \max 20}$.

If voxels are assumed to obey Bragg-Gray conditions when calculating $\mathrm{D}_{w}$, the results in Figure 8.1(c) indicate that $\mathrm{D}_{w}$ is similar to $\mathrm{D}_{m}$ in all soft tissue, but in bone it is $15-20 \%$ higher (maximum values of $115-120 \%$ of $\mathrm{D}_{m, \max 20}$ in pelvis and sternum, $90-95 \%$ of $\mathrm{D}_{m, \max 20}$ in spine). This is expected given that, from Figure 8.2(a), $\bar{S}_{b o n e}^{w}$ is in the range 1.15-1.20. Within the rest of the phantom, Figure 8.2(a) shows that $\bar{S}_{m}^{w}$ is within $5 \%$ of unity, and, therefore, will not result in values of $\mathrm{D}_{w}$ different enough from $\mathrm{D}_{m}$ to be visible given the dose resolution of Figure 8.1. Note that the misassignment of adipose tissue to lung (see above) results in $\bar{S}_{m}^{w}$ values in the range 0.95-0.98, which is significantly lower than the estimated value for lung of 1.009 shown in Table 8.2 but encompasses the value of 0.963 estimated for adipose tissue.

If CPE is assumed to prevail in the voxels, then Figure 8.1(d) shows that $\mathrm{D}_{w}$ in bone is $50-60 \%$ lower than $\mathrm{D}_{m}$, with $\mathrm{D}_{w}$ equal to $40-50 \%$ of $\mathrm{D}_{m, \text { max } 20}$ in the pelvis and sternum and $30-40 \%$ of $\mathrm{D}_{m, \max 20}$ in the spine. On the other hand, $\mathrm{D}_{w}$ in tissue near the beam entry surface is higher than $\mathrm{D}_{m}$, with $\mathrm{D}_{w}$ equal to $30-40 \%$ of $\mathrm{D}_{m, \max _{20}}$ where $\mathrm{D}_{m}$ is $25-30 \%$ of $\mathrm{D}_{\operatorname{m}, \max 20}$. From Figure $8.2(\mathrm{~b})$, the values of $\left[\frac{\overline{\mu_{e n}}}{\rho}\right]_{m}^{w}$ in bone are in the range $0.4-0.5$, accounting for the $50-60 \%$ difference between $\mathrm{D}_{m}$ and $\mathrm{D}_{w}$ in these regions. The values of $\left[\frac{\overline{\mu_{e n}}}{\rho}\right]_{m}^{w}$ in the adipose tissue near the beam entry surface and in the misassigned lung are in the range 1.05-1.1, resulting in $\mathrm{D}_{w}$ being visibly higher than $\mathrm{D}_{m}$ in these regions. Elsewhere, in muscle, $\left[\frac{\overline{\mu_{e n}}}{\rho}\right]_{m}^{w}$ is very close to unity, resulting in no discernible difference between $\mathrm{D}_{w}$ and $\mathrm{D}_{m}$.

For the media distinguishable in this phantom-adipose tissue, muscle and bone-the values of $\bar{S}_{m}^{w}$ and $\left[\frac{\overline{\mu_{e n}}}{\rho}\right]_{m}^{w}$ estimated separately using SPRRZnrc and $\mathrm{g}$, shown in Table 8.2, fall within their respective ranges in Figure 8.2, providing some confidence in these latter values, which are calculated on-the-fly during the simulation.

A simplified perspective on the $\mathrm{D}_{w}$ results in Figure 8.1 is that Figure 8.1(b) is a weighted sum of Figures 8.1(c) and (d). Considering smaller cavity dimensions (larger values of $d$ ) will result in calculated $\mathrm{D}_{w}$ values closer to those in Figures 8.1(c), while larger cavity dimensions (smaller values of $d$ ) will move results closer to those in Figures 8.1(d). From this perspective, the potential for cavity dimension to have a clinically significant impact on $\mathrm{D}_{w}$ is obvious. The effects will be greatest in bone, but may also occur in regions of adipose tissue.

The limited dose resolution in Figure 8.1 resists a more detailed analysis. However, this example illustrates that consideration of the intermediate size of cavities in kilovoltage beams does have a clinically significant impact on the estimated value of $\mathrm{D}_{w}$. Also, determining the factor, $d$, used to weight $\mathrm{BG}$ and CPE assumptions and, thus, account for intermediate dimensions, is complicated by: 1) a lack of agreement on the best method to calculate it and 2) uncertainty over a meaningful cavity dimension.

Beyond cavity size considerations, there are other, less tractable issues, complicating the conversion of $\mathrm{D}_{m}$ to $\mathrm{D}_{w}$. It is acknowledged[25,43,44] that the conversion of CT number to medium represents a weak link in MC RTP, with possible variations between institutions and imagers that depend on the conversion function and number of media used. Also, lacking patient-specific data, most researchers use the tissue compositions given in the most recent reports by the ICRU[45] and ICRP[46]. It is cer- 
tain, however, that composition varies from patient to patient[43], and Andreo shows that the resultant variation in mean excitation energy has a significant impact on the water-to-medium stopping power ratio[25].

To introduce yet another consideration into this multifaceted debate, current research [32, 38, 47] posits that, since interactions resulting in RBE happen in DNA-bound water existing in $\sim \mathrm{nm}$-size volumes, a value of $\mathrm{D}_{w}$ with any biological meaning would be arrived at using strictly BG cavity theory, even for low-energy beams. On the other hand, in their MC study, Oliver and Thomson[38] found that $\mathrm{D}_{m}$ in small $(\mathrm{r}<10 \mu \mathrm{m})$ volumes is dependent on the composition and structure of the surrounding media, potentially rendering $\mathrm{D}_{w}$, which is generally based on $\mathrm{D}_{m}$ in bulk media, clinically meaningless until the effects of tissue composition are better understood.

\subsubsection{Implications of efficient MC dose calculations for commercial treatment planning systems}

The research presented in this thesis touches on only a small fraction of the past and current work developing MC simulations for radiotherapy and radiation protection applications, reflecting the author's direct involvement in specific areas of code development and use. It is also heavily skewed towards applications of the EGSnrc system which, while currently the most widely-used MC code for radiotherapy/radiation protection research, it must be acknowledged is only one player among a number that has grown over the past decade and a half. Even from this circumscribed vantage point, however, two broad observations can be made: 1) Improvements in the efficiency of treatment head simulation and dose calculation algorithms, together with increases in computing speed, have contributed to making clinical MC treatment planning a reality and 2) The information available from MC simulations has increased our understanding of radiotherapy, with the potential of allowing treatment protocols to be tailored from accelerator design to RBE.

In addition, MC has demonstrated an ability to keep pace with significant developments in radiotherapy, such as IMRT[48] and IGRT[49], continuing to provide researchers with the most accurate method of calculating dose. From 1985 to the late 2000's, the number of research publications related to MC treatment planning increased exponentially[50], and while this certainly reflects an inevitable increase in the number of researchers in the field, it must also indicate the increasing number of potential applications of MC and a growing consensus that it is the preferred method for addressing issues of radiation treatment protocol design and optimization.

Given this, it is no surprise that there has been a push to adopt MC codes in clinical practice, with PEREGRINE[51], the first commercially-available photon treatment planning system incorporating MC (using a fast variant of EGS4), achieving FDA approval in 2000. A survey by Reynaert et al[52] at the end of 2004 showed eight commercial TPSs using MC either available (Corvus and Eclipse ) or in the late stages of development. In 2007, the report of AAPM Task Group 105[44] anticipated an increase in number of commercially available MC treatment planning systems, suggesting a series of protocols for commissioning systems and noting the imminent development of several more commercial systems 
making use of MC. In their 2008 review, Spezi and Lewis[50] portray an increasingly diverse and dynamic array of research treatment planning systems based on EGSnrc, Penelope, Geant4, and other MC codes, but note, by contrast, the relatively modest inroads into the commercial realm, with only three commercially available systems incorporating MC dose calculations at that time.

To date, implementation of MC in commercially available TPSs continues at a rate that seems at odds with its potential and with the concurrent level of research activity. In a recent review, Brualla et al[53] enumerate only five commercially available, FDA-approved external beam TPSs making use of MC simulations. These are (along with the MC code used): Varian Eclipse (MMC[54]), Brainlab iPlan (XVMC), Elekta Monaco (XVMC), Elekta Oncentra (VMC++, a development of XVMC with enhanced variance reduction), and Elekta XiO ${ }^{\circ}$ (XVMC). Note that PEREGRINE has been discontinued and that Varian Eclipse, one of the vanguard MC TPSs, uses an algorithm for electron dose (MMC), which is over twenty years old. One can add to this list Raysearch's Raystation , which uses VMC[55] to calculate electron dose. In all cases, these systems make use of a fast MC dose calculation algorithm (a dose "engine," to use their terminology) to calculate either photon or electron dose, but not both. The speed of these codes necessitates the use of virtual source models, simplified parameterizations of the linac, based on either phase space data generated by an MC simulation or on measurements, in order to avoid the potential computational bottleneck of delivering source particles from a linac simulation or sampling phase space data, mentioned in section 8.1 .1 above. Inaccuracies introduced by even well-tuned virtual source models may be up to $2 \%[56]$, which, while smaller than the acceptable clinical uncertainty of 5\%[44], would seem to work against the potential accuracy achievable by MC. Such inaccuracies may particularly affect electron dose calculations, in which dose attains a maximum near the patient/phantom surface, where systematic uncertainties in the source will have the greatest effect. Moreover, there is a tradeoff in flexibility and generality when using virtual source models specifically tuned to manufacturer beam specifications.

Potential limitations of virtual source models reflect just one of a number of more general issues contributing to the slow entry of MC into commercially available TPSs. Among these more general issues are:

- The requirement for expertise: While commercial MC codes are relatively streamlined compared to their general purpose counterparts (e.g., EGSnrc, PENELOPE, Geant4), a level of MC expertise is still desirable when interpreting results for a given set of inputs. In addition, commercial code development is informed by general purpose MC codes, and the open-ended nature of development of the latter-including the multiplication of input options as transport physics is refined and variance reduction techniques are developed-contributes to a perception that expertise is required to run all MC codes with any confidence.

- Newer treatment modalities: Currently, there is growing interest in proton beam therapy, with its promise of greater accuracy and reduced damage to normal tissue due to the fact that maximum energy is deposited in a relatively narrow Bragg peak. Clinics in the developed world are now making huge investments in proton therapy facilities. Carbon ions, too, have been the sub- 
ject of increasing interest as their high linear energy transfer (LET) results in greater localization of $\mathrm{RBE}$ at depth than protons. The rapid increase in development of these modalities has not been matched by the development of accurate and efficient $\mathrm{MC}$ algorithms. With the exception of VMCpro[57], an efficient proton beam dose calculation algorithm developed in 2004, simulation of protons requires relatively inefficient general purpose codes, such as MCNP, Geant4 and FLUKA[58], capable of simulating all particles. In a 2012 review article, Paganetti[59] concluded that regular clinical use of $\mathrm{MC}$ for proton beam treatment planning would only result in a clinically-significant increase in accuracy over current pencil beam algorithms in complex geometries. While this might dampen initial enthusiasm for the use of MC in proton beam therapy, recall that one of the arguments for the use of $\mathrm{MC}$ for photon and electron beam dose calculations was its ability to handle just such complexities. As for carbon ion beams, while there has been progress in efficient MC simulation[60], recent research also suggests significant improvements in nuclear fragmentation models are required[61].

- Other sources of uncertainty: Despite the sophistication of MC dose calculation algorithms, there remain intrinsic sources of uncertainty in the MC treatment planning chain that compromise the accuracy of the calculated dose. Among these are the systematic uncertainties in the virtual source model of the incident beam, mentioned above, and uncertainties in the CT-to-medium conversion, mentioned in section 8.1.3. In the eyes of clinicians and commercial developers, such issues may weigh against the obvious advantages of $\mathrm{MC}$ treatment planning algorithms over traditional pencil beams. Uncertainty in cross-sections is also a source of uncertainty in MC treatment planning, but at a level far below clinical significance.

- Statistical overkill: Provided that all inputs are correct, MC treatment planning promises dose accuracies on the order of $1 \%$. On the other hand, it is generally accepted[44] that clinically significant differences in tumour control probability (TCP) and normal tissue complication probability (NTCP) are likely only at the 5-7\% level. Thus, the cost-effectiveness of implementing MC treatment planning may come into question. While few would argue against greater precision and accuracy of delivery of ionizing radiation on the basis of safety, there are currently no longitudinal studies clearly showing the medical benefits of the MC treatment planning accuracy.

Another perspective on the development of MC TPSs may be gained by comparison with the approximate development timelines of analogous refinements of existing techniques for cancer therapy, from discovery, theory, or proof-of-concept to FDA approval. A small selection of these, along with the timeline for MC RTP is shown in Figure 8.3. Note that the starting years of the timelines are approximate and, moreover, open to debate. In the case of MC RTP, the chosen year (1985) coincides with the publication of EGS4 by Nelson et al[62], as this is acknowledged among most researchers to mark the first serious application of MC to radiotherapy on a scale beyond individual institutions.

From this figure, it is evident that the total time from "initial" development to FDA approval of the first MC RTP system (PEREGRINE) is in keeping with that of other techniques used to improve or augment existing cancer treatment methods. However, while the timeline for MC RTP comprises 


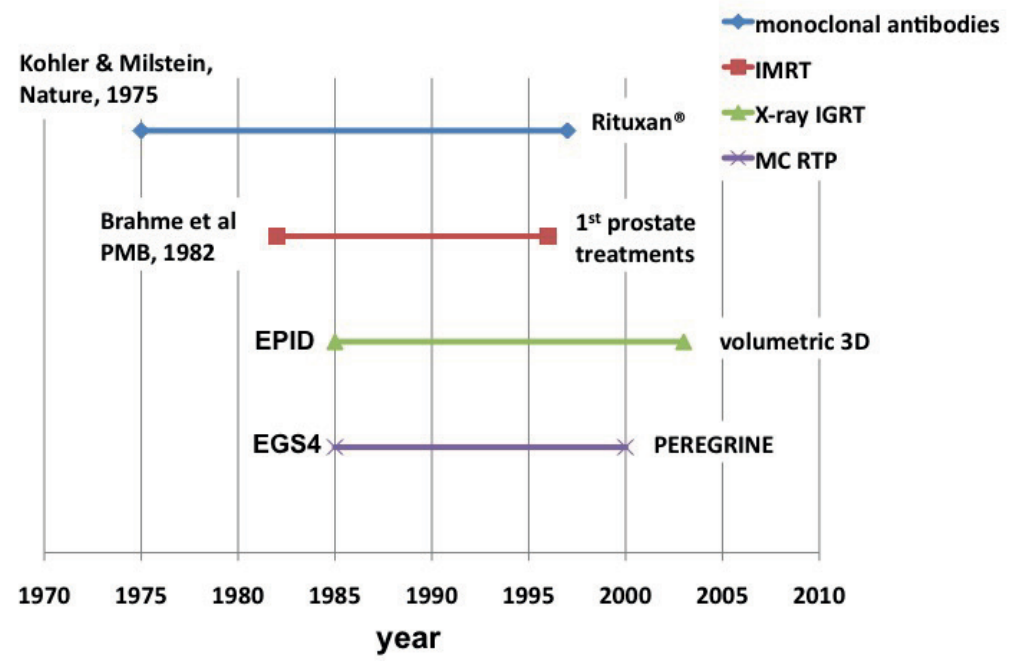

Figure 8.3: Timelines for development-initiation to FDA approval-of augmentative techniques for cancer treatment. For monoclonal antibodies, the time spans the documentation of the first monoclonal antibody by Kohler and Milstein in 1975 to FDA approval of Rituxan , the first monoclonal antibody approved for the treatment of cancer (non-Hodgkin's lymphoma) in 1997[63]. For intensity modulated radiotherapy (IMRT), the time spans the publication of Brahme et al's $2 \mathrm{D}$ inverse solution to the first regular treatments of prostate cancer using IMRT in 1996[64]. For X-ray image-guided radiotherapy (IGRT), the timeline spans initial development of the first planar electronic portal imaging devices (EPIDs) for MV imaging to regular clinical use of 3D volumetric imaging with cone beam CT (CBCT)[65]. The development of MC radiotherapy treatment planning (MC RTP) is considered to begin with the publication of the EGS4 code by Nelson et al in 1985[62].

a combination of refinement of techniques and parallel (and unrelated) advances in computing power, that for IMRT involves the developent of dedicated hardware and software, while the timeline for X-ray IGRT encompasses the development of an entirely new imaging modality (cone beam CT). Considering, then, the relatively minor commercial interest in MC RTP compared to these other technologies, one might conclude that it has also to do with its low visibility in comparison to the excitement inevitably accompanying the emergence of new large-scale hardware.

\subsubsection{Summary and Future Work}

This thesis describes research into variance reduction techniques that have led to significant increases in efficiency in Monte Carlo (MC) calculated photon dose over the last two decades. Also presented is a thorough analysis of the history by history statistical method which is essential for estimating the uncertainty on calculated doses and, by extension, determining the efficiency of these variance reduction techniques. 
One result of this research has been the ability to perform dose calculations in simulated human phantoms under realistic treatment conditions. Two such studies in voxellized human phantoms are presented in which dose to sensitive tissues in bone (red bone marrow and bone surface cells) is used to: a) assess the impact of additional radiation from CBCT imaging during image-guided radiotherapy (IGRT) and b) determine whether dose to medium, $\mathrm{D}_{m}$, calculated by MC algorithms, or dose to waterin-medium, $\mathrm{D}_{w}$, typically specified by most treatment planning systems, is a better estimate of the dose to these sensitive tissues in megavoltage photon beams. It is found that, in general, the difference is clinically insignificant, except in cranial bone, where dose to water is more accurate.

While the goal is to specify the dose that is the best predictor of radiobiological effect (RBE), the conversion of $\mathrm{D}_{m}$ to $\mathrm{D}_{w}$ is not straightforward, especially in the $\mathrm{kV}$ range used in brachytherapy and small animal irradiators. The Discussion delves into this further, presenting Burlin's cavity theory, which other researchers have suggested may be used for intermediate-size cavities in which neither Bragg-Gray conditions nor charged particle equilibrium prevail. In this case, the conversion factor for $\mathrm{D}_{m}$ to $\mathrm{D}_{w}$ is a weighted sum of the water-to-medium stopping power ratio and the water-to-medium ratio of mass energy transfer coefficients. New results are shown for the application of Burlin cavity theory to a simulated irradiation ( $225 \mathrm{kVp}$ beam) of a mouse, showing that conversion using only stopping power ratios results in an overestimate of $\mathrm{D}_{w}$ in bone, while conversion using only the ratios of mass-energy transfer coefficients results in an underestimate.

The Discussion also presents a brief summary of the current state of MC in commercially available treatment planning systems, noting that the inroads made by the technology in the clinic do not appear to be commensurate with research activity. There are several reasons for this. However, the dominant factor may be that the economics of achieving $1 \%$ accuracy in dose has not yet been justified. Moreover, despite the many recent advances in variance reduction, CPU times required to obtain dose uncertainties $<2 \sigma$ of this accuracy remain an issue.

Commercially, it is difficult to conclude whether the current small number of available MC treatment planning systems represents the first, tentative steps of a growing trend or one among many technologies ultimately superceded on the road to better treatment planning. Certainly one factor working against its widespread use is the necessarily-conservative realm of medical device research, testing and ultimate FDA approval. Historically, however, disruptive technologies have been adopted by the medical community provided that they result in a demonstrable benefit. In addition to addressing the more technical issues mentioned in the Discussion, then, there is motivation for more longitudinal evidence clearly showing the medical benefits of the level of accuracy that MC treatment planning promises.

By contrast, on the research and development side, the rise of new treatment modalities is likely to see an expansion of the role of MC in the commissioning and verification of treatment beams and TPSs. As mentioned above, this is already happening in proton therapy research. In the short term, increased interest in heavy ion therapy is expected to result in a push for development or refinement of models for nuclear fragmentation in general purpose MC codes. In addition, innovations in photon and electron beam therapy will continue to influence MC code development. For example, the clinical advent of MRIbased IGRT has sparked a renewed interest in developing and benchmarking MC routines for charged 
particle transport in the presence of magnetic fields, such as that recently implemented in EGSnrc by Malkov and Rogers[66].

EGSnrc is likely to retain its status as the most-used general purpose MC code for radiation protection and radiotherapy research for some time, given the code's extensive history and its efficiency for photon and electron beam simulation. However, its utility in the long term will depend on its adaptability. While the core of EGSnrc (and many applications) is coded in Mortran, an extension of Fortran dating back to the early 1980's, current development of EGSnrc applications makes use of the egspp class library[67], a C++ object-oriented geometry and source library. It would be a desirable "outside in" evolution if the transport physics in EGSnrc underwent an object-oriented overhaul and was reprogrammed using $\mathrm{C}++$ classes. This would make the addition of new modules simulating, for example, proton or heavy ion physics a much more feasible (and less daunting) prospect. It would also make the code more transparent and amenable to contributions from outside the development team. Considering the significant contributions already made by the users community, one can only imagine where the medical physicists of the future will take EGS as they advance the forefront of the field.

\subsection{Bibliography}

[1] B. R. B. Walters, I. Kawrakow, and D. W. O. Rogers. History by history statistical estimators in the BEAM code system. Med. Phys., 29:2745 - 2752, 2002.

[2] Iwan Kawrakow, D. W. O Rogers, and B.R.B. Walters. Large efficiency improvements in BEAMnrc using directional bremsstrahlung splitting. Med. Phys., 31:2883 - 2898, 2004.

[3] J. Briesmeister. MCNP-A general purpose Monte Carlo code for neutron and photon transport, Version 3A. Los Alamos National Laboratory Report LA-7396-M (Los Alamos, NM), 1986.

[4] J. Sempau, E. Acosta, J. Baro, J. M. Fernandez-Varea, and F. Salvat. An algorithm for Monte Carlo simulation of coupled electron-photon transport. Nucl. Inst. Meth. B, 132, 1997.

[5] S. Agostinelli et al. GEANT4 - a simulation toolkit. Nucl. Inst. Meth. A, 506:250 - 303, 2003.

[6] R. Capote, R. Jeraj, C.-M. Ma, D.W.O. Rogers, F. Sanchez-Doblado, J. Sempau, J. Seuntjens, and J.V. Siebers. Phase-Space Database for External Beam Radiotherapy. IAEA Report INDC(NDS)-0484, 2005.

[7] I. Kawrakow and B. R. B. Walters. Efficient photon beam dose calculations using DOSXYZnrc with BEAMnrc. Med. Phys., 33:3046 - 3056, 2006.

[8] J. Lobo and I. A. Popescu. Two new dosxyznrc sources for $4 \mathrm{~d}$ monte carlo simulations of continuously variable beam configurations, with applications to rapidarc, vmat, tomotherapy and cyberknife. Phys. Med. Biol., 55:4431 - 4443, 2010. 
[9] R. W. Townson, X. Jia, Z. Tian, Y. J. Graves, S. Zavgorodni, and S. B. Jiang. GPU-based Monte Carlo radiotherapy dose calculation using phase-space sources. Phys. Med. Biol., 58:4341, 2013.

[10] I. A. Popescu, P. Atwal, J. Lobo, J. Lucido, and B. M. C. McCurdy. Patient-specific QA using 4D Monte Carlo phase space predictions and EPID dosimetry. Journal of Physics: Conference Series, 573:012004, 2015.

[11] S. Yani, I. G. E. Dirgayussa, M. F. Rhani, F. Haryanto, and I. Arif. Monte Carlo investigation of inhomogeneity phantom effects in $6 \mathrm{MV}$ photon beam. Transactions on Science and Technology, 3: 336 - 344, 2016.

[12] A. Nevelsky, E. Borzov, S. Daniel, and R. Bar-Deroma. Room scatter effects in Total Skin Electron Irradiation: Monte Carlo simulation study. Journal of Applied Clinical Medical Physics, 18:196 - 201, 2017.

[13] T. Teke, C. Duzenli, A. Bergman, F. Viel, P. Atwal, and E. Gete. Monte Carlo validation of the TrueBeam 10XFFF phase-space files for applications in lung SABR. Med. Phys., 42:6863 - 6874, 2015.

[14] https://varian.force.com/.

[15] I. Kawrakow and M. Fippel. VMC++, a fast MC algorithm for radiation treatment planning. In W. Schlegel and T. Bortfeld, editors, The Use of Computers in Radiotherapy, XIIIth Int'l Conf., Heidelberg, pages 126 -128. Springer-Verlag, Heidelberg, 2000.

[16] M. Fippel. Fast Monte Carlo dose calculation for photon beams based on the VMC electron algorithm. Med. Phys., 26:1466 - 1475, 1999.

[17] Federico Hasenbalg, Michael Fix, Ernst Born, Roberto Mini, and Iwan Kawrakow. VMC++ versus BEAMnrc: a comparison of simulated linear accelerator heads for photon beams. Med. Phys., page in press, 2008.

[18] E. Mainegra-Hing and I. Kawrakow. Efficient x-ray tube simulations. Med. Phys., 33:2683 - 2690, 2006.

[19] B. R. B. Walters. Increasing efficiency of BEAMnrc-simulated Co-60 beams using directional source biasing. Med. Phys., 42:5817 - 5827, 2015.

[20] F. Verhaegen, A. E. Nahum, S. Van de Putte, and Y. Namito. Monte Carlo modelling of radiotherapy kV x-ray units. Phys. Med. Biol., 44:1767 - 1789, 1999.

[21] B. R. B. Walters, G. X. Ding, R. Kramer, and I. Kawrakow. Skeletal dosimetry in cone beam computed tomography. Med. Phys., 36:2915 - 2922, 2009. 
[22] B. R. B. Walters, R. Kramer, and I. Kawrakow. Dose to medium versus dose to water as an estimator of dose to sensitive skeletal tissue. Phys. Med. Biol., 55:4535 - 4546, 2010.

[23] J. V. Siebers, P. J. Keall, A. E. Nahum, and R. Mohan. Converting absorbed dose to medium to absorbed dose to water for Monte Carlo based photon beam dose calculation. Phys. Med. Biol., 45: $983-995,2000$.

[24] N. Dogan, J. V. Siebers, and P. J. Keall. Clinical comparison of head and neck and prostate IMRT plans using absorbed dose to medium and absorbed dose to water. Phys. Med. Biol., 51:4967-4980, 2006.

[25] P. Andreo. Dose to fhwater-likefh media or dose to tissue in MV photons radiotherapy treatment planning: still a matter of debate. Phys. Med. Biol., 60:309 - 337, 2015.

[26] R. Nath, L. L. Anderson, G. Luxton, K. A. Weaver, J. F. Williamson, and A. S. Meigooni. Dosimetry of interstitial brachytherapy sources: Recommendations of the AAPM Radiation Therapy Committee Task Group No. 43. Med. Phys., 22:209 - 234, 1995.

[27] M. J. Rivard, B. M. Coursey, L. A. DeWerd, M. S. Huq, G. S. Ibbott, M. G. Mitch, R. Nath, and J. F. Williamson. Update of AAPM Task Group No. 43 Report: A revised AAPM protocol for brchytherapy dose calculations. Med. Phys., 31:633 - 674, 2004.

[28] V. Gimènez-Alventosa, P. C. G. Antunes, J. Vijande, F. Ballester, J. Pérez-Calatyud, and P. Andreo. Collision-kerma conversion between dose-to-tissue and dose-to-water by photon energy-fluence corrections in low-energy brachytherapy. Phys. Med. Biol., 62:146 - 164, 2017.

[29] G. P. Fonseca, A. Carlsson Tedgren, B. Reniers, J. Nilsson, M. Persson, H. Yoriyaz, and F. Verhaegen. Dose specification for ${ }^{192}$ Ir high dose rate brachytherapy in terms of dose-to-water-in-medium and dose-to-medium-in-medium. Phys. Med. Biol., 60:4565 - 4579, 2015.

[30] F. Verhaegen, P. Granton, and E. Tryggestad. Small animal radiotherapy research platforms. Phys. Med. Biol., 56:R55 - R83, 2011.

[31] K. T. Butterworth, K. M. Prise, and F. Verhaegen. Small animal image-guided radiotherapy: status, considerations and potential for translational impact. British Journal of Radiology, 88:20140634, 2015.

[32] A. Carlsson Tedgren and G. Alm Carlsson. Specification of absorbed dose to water using modelbased dose calculation algorithms for treatment planning in brachytherapy. Phys. Med. Biol., 58: 2561 - 2579, 2013.

[33] T. E. Burlin. A general theory of cavity ionization. Br. J. Radiol., 39:727 - 734, 1966.

[34] P. N. Mobit, P. Mayles, and A. E. Nahum. The quality dependence of of LiF TLD in megavoltage photon beams: Monte Carlo simulations and experiments. Phys. Med. Biol., 41:387 - 398, 1996. 
[35] T. E. Burlin and F. K. Chan. The Effect of the Wall on the Fricke Dosimeter. Int'l J App Rad Isotop, 20:767 - 775, 1969.

[36] A. Janssens, G. Eggermont, R. Jacobs, and G. Thielens. Spectrum perturbation and energy deposition models for stopping power ratio calculations in general cavity theory. Phys. Med. Biol., 19:619 $-627,1974$.

[37] R. Loevinger. The dosimetry of beta sources in tissue: the point-source function. 66:55 - 62, 1956.

[38] P. A. K. Oliver and R. M. Thomson. Cavity theory applications for kilovoltage cellular dosimetry. Phys. Med. Biol., 62:4440 - 4459, 2017.

[39] G. Poludniowski, G. Landry, F. DeBlois, P. M. Evans, and F. Verhaegen. SpekCalc: a program to calculate photon spectra from tungsten anode x-ray tubes. Phys. Med. Biol., 54:N433 - N438, 2009.

[40] ICRU. Stopping powers for electrons and positrons. ICRU Report 37, ICRU, Washington D.C., 1984.

[41] L. V. Spencer and F. H. Attix. A theory of cavity ionization. Radiat. Res., 3:239 - 254, 1955.

[42] D. W. O. Rogers, I. Kawrakow, J. P. Seuntjens, B. R. B. Walters, and E. Mainegra-Hing. NRC User Codes for EGSnrc. Technical Report PIRS-702(RevC), National Research Council of Canada, Ottawa, Canada, 2010.

[43] H. H. Liu and P. Keall. Point/Counterpoint: $\mathrm{D}_{m}$ rather than $\mathrm{D}_{w}$ should be used in Monte Carlo treatment planning. Med. Phys., 29:922 - 924, 2002.

[44] I. J. Chetty et al. Report of the AAPM Task Group No. 105: Issues associated with clinical implementation of Monte Carlo-based treatment planning. Med. Phys., 34:4818-4853, 2007.

[45] ICRU. Tissue Substitutes in Radiation Dosimetry and Measurements. ICRU Report 44, ICRU, Washington D.C., 1989.

[46] ICRP. Basic Anatomical and Physiological Data for Use in Radiological Protection: Reference Values. Annals of the ICRP 89, ICRP, Washington D.C., 2002.

[47] P. A. K. Oliver and R. M. Thomson. A Monte Carlo study of macroscopic and microscopic dose descriptors for kilovoltage cellular dosimetry. Phys. Med. Biol., 62:1417 - 1437, 2017.

[48] J. O. Deasy, E. Lee, I. Kawrakow, and C. Zakarin. A prototype monte carlo based imrt treatment planning research system. Med. Phys. (abstract), page 1254, 2002.

[49] P. J. Keall, J. V. Siebers, S. Joshi, and R. Mohan. Monte Carlo as a four-dimensional radiotherapy treatment-planning tool to account for respiratory motion. Phys. Med. Biol., 49:3639, 2004. 
[50] E. Spezi and G. Lewis. An overview of Monte Carlo treatment planning for radiotherapy. Radiation Protection Dosimetry, 131:123 - 129, 2008.

[51] C. L. Hartmann-Siantar et al. Lawrence Livermore National Laboratory's PEREGRINE project. In "Proceedings of the XII-th Conference on the Use of Computers in Radiotherapy" (Medical Physics Publishing, Madison, Wisconsin), pages 19 - 22, 1997.

[52] N. Reynaert, S. C. van der Marck, D. R. Schaart, W. Van der Zee, C. Van Vliet-Vroegindeweij, M. Tomsej, J. Jansen, B. Heijmen, M. Coghe, and C. De Wagter. Monte Carlo treatment planning for photon and electron beams. Radiation Physics and Chemistry, 76:643 - 686, 2007.

[53] L. Brualla, M. Rodriguez, and A. M. Lallena. Monte Carlo systems used for treatment planning and dose verification. Strahlentherapie und Onkologie, 193:243 - 259, 2017.

[54] H. Neuenschwander, T. R. Mackie, and P. J. Reckwerdt. MMC-A high-performance Monte Carlo code for electron beam treatment planning. Phys. Med. Biol., 40:543 - 574, 1995.

[55] I. Kawrakow, M. Fippel, and K. Friedrich. 3D Electron Dose Calculation using a Voxel based Monte Carlo Algorithm (VMC). Med. Phys., 23:445 - 457, 1996.

[56] Matthias Fippel, Freddy Haryanto, Oliver Dohm, Fridtjof Nusslin, and Stephan Kriesen. A virtual photon energy fluence model for Monte Carlo dose calculation. Med. Phys., 30:301-311, 2003. http://link. aip.org/link/?MPH/30/301/1.

[57] Matthias Fippel and Martin Soukup. A Monte Carlo dose calculation algorithm for proton therapy. Med. Phys., 31:2263-2273, 2004. http://link.aip.org/link/?MPH/31/2263/1.

[58] A. Fasso, A. Ferrari, J. Ranft, and P. R. Sala. FLUKA: Present status and future developments. Proceedings of the 4th Conference on calorimetry in High Energy Physics, World Sci., Singapore:493 $-502,1994$.

[59] H. Paganetti. Range uncertainties in proton therapy and the role of Monte Carlo simulations. Phys. Med. Biol., 57:R99 - R117, 2012.

[60] N. Qin, M. Pinto, Z. Tian, G. Dedes, A. Pompos, S. Jiang, K. Parodi, and X. Jia. OpenCL-Based Cross-Platform Monte Carlo Simulation Package for Carbon Ion Therapy. Med. Phys., 43:3318, 2016.

[61] C. Robert et al. Distributions of secondary particles in proton and carbon-ion therapy: a comparison between GATE/Geant4 and FLUKA Monte Carlo codes. Phys. Med. Biol., 58:2879 - 2899, 2013.

[62] W. R. Nelson, H. Hirayama, and D. W. O. Rogers. Electron Photon Transport using the EGS4 Monte Carlo Code. Transactions of the American Nuclear Society, 52:379 - 380, 1986. 
[63] M. Stern and R. Herrmann. Overview of monoclonal antibodies in cancer therapy: present and promise. Hematology, 54:11 - 29, 2005.

[64] S. Webb. The physical basis of IMRT and inverse planning. The British Journal of Radiology, 76: 679-689, 2003.

[65] D. Verellen, M. De Ridder, and G. Storme. A (short) history of image-guided radiotherapy. Radiotherapy and Oncology, 86:4 - 13, 2008.

[66] V. N. Malkov and D. W. O. Rogers. Charged particle transport in magnetic fields in EGSnrc. Med. Phys., 43:4447 - 4458, 2016.

[67] I. Kawrakow. egspp: the EGSnrc C++ class library. Technical Report PIRS-899, National Research Council of Canada, Ottawa, Canada, 2005. 

Appendices 



\section{Summary}

Since the mid-80's, it has been acknowledged that Monte Carlo (MC) transport algorithms provide the most general and most accurate method of estimating dose due to ionizing radiation. This is because MC algorithms model individual interactions between subatomic particles and matter, allowing accurate dose calculations at interfaces between media having a large difference in atomic number (e.g., tissue-bone interfaces) and in irregular geometries, both instances where the analytical algorithms commonly used in treatment planning are known to be inaccurate. In addition, MC simulations can provide information unavailable with current analytical methods, such as particle energy and angular distribution at any location within a treatment volume. Historically, the long calculation times required to obtain sufficient precision have made the routine use of MC methods in radiotherapy treatment planning (RTP) clinically impractical. Recent improvements in efficiency of MC techniques, including the development of dedicated clinical MC algorithms, together with the ongoing exponential increase in computing power, however, have rendered clinical MC calculations feasible. The research presented in this thesis covers several key efficiency improvements in EGSnrc, a well-benchmarked general purpose MC transport simulation code commonly used in Medical Physics research, and two practical applications of EGSnrc answering questions about dose to sensitive red bone marrow and bone surface cells in the skeletal system. The first paper describes the implementation of an improved technique for estimating uncertainty on calculated dose, and other scored quantities, in EGSnrc applications. The technique considers each incident primary particle, or history, a statistically independent event, resulting in a small variance in the uncertainty estimate. This has many implications, among them an ability to accurately calculate simulation efficiency that has helped pave the way for the efficiency improvement techniques described in the next three chapters. The first of these techniques is a time-saving algorithm, called "HOWFARLESS," implemented in DOSXYZnrc, an EGSnrc application for calculating dose in geometries composed of rectilinear voxels. The research shows that use of "HOWFARLESS" can result in efficiency improvements by factors ranging from $70 \%$ to $17 \times$ in routine commissioning calculations, essentially making it the default for 
such calculations in a homogeneous volume. A common technique to reduce the variance, and increase the efficiency, of an MC calculation is to split certain particle interactions. This results in improved sampling of these events and, provided the resultant split products of the interaction do not deposit dose in the same volume, improved statistics. The third paper describes the technique of directional bremsstrahlung splitting (DBS) implemented in BEAMnrc, an EGSnrc application for simulating linear accelerators. In DBS, bremsstrahlung events, which are the primary sources of photons in a photon treatment beam, are split to selectively generate a large number of photons directed towards the radiotherapy treatment field. The paper shows that DBS can increase dose calculation efficiency by a factor of $160 \times$. Improvements are even greater for X-ray tube simulations, and DBS is now considered necessary for efficient simulation of photon beams, in general. The next paper details the implementation in BEAMnrc of directional source biasing (DSB), a splitting technique designed to improve the efficiency of treatment beams making use of an isotropically radiating source. The most common example of this is a cobalt-60 treatment head, and, while cobalt- 60 is one of the oldest radiotherapy modalities, there has been a resurgence in its use due to its cost-effectiveness and the relative ease with which it can be adapted for use with magnetic resonance imaging for image-guided radiotherapy (IGRT). Similar to directional bremsstrahlung splitting, DSB splits photons and selectively generates those directed towards the treatment field. In the case of DSB, however, these photons are the primary particles, generated at the source, and have an isotropic angular distribution. The paper shows that DSB can increase the efficiency of a cobalt- 60 dose calculation by $400 \times$, making routine MC commissioning of these units feasible. The last two papers in this thesis illustrate how efficient MC photon beam simulations, combined with MC calculations of dose in human phantoms, can be used to obtain clinicallyrelevant information about dose to radiosensitive tissue in the human skeleton. The human phantoms are constructed from CT image data and have the useful feature that computed dose to skeletal spongiosa, the portion of bone within which blood cells originate, can be resolved into dose to its radiosensitive components: red bone marrow and bone surface cells. In the first of these papers, simulation of routine cone-beam CT imaging during IGRT demonstrates that the additional dose to bone surface cells from the imaging procedure may be a significant fraction of the overall dose received by radiosensitive bone tissue, thus motivating imaging guidelines. Research in the final paper shows that dose expressed as dose to water-in-medium, the output from commercially available treatment planning systems, is, in general, a more accurate estimate of dose to red bone marrow and bone surface cells than dose expressed as dose to medium, the standard output from MC dose calculation algorithms. Thus, in the case of the latter dose computation, conversion to dose to water-in-medium would seem to be justified. 
However, there is ongoing debate over which method of dose specification is a more accurate reflection of radiobiological effects. Moreover, conversion to dose to water-in-medium is not straightforward, particular at lower photon energies, and depends upon the effective volume considered. New research in the Discussion section of this thesis provides a quantitative and qualitative illustration of the effect of the particular cavity theory used to perform this dose conversion. 



\section{Acknowledgements}

I would like to acknowledge my colleagues, past and present, at the National Research Council for cultivating a research environment in which work, play and creativity are allowed to flourish in equal measure. I know better than to hope I'll ever experience such a workplace again. I would especially like to thank Dr. Ernesto Mainegra-Hing, my comrade at arms in the Monte Carlo trenches for almost two decades. And, in case it's not evident from the lists of authors, I wish to acknowledge Dr. Iwan Kawrakow, with the help of whose tenacity I was able to continue publishing papers at a rate of $\sim 1$ year and whose vision provided guidance for much of this research. I also owe debts of gratitude to Dr. Frank Verhaegen and Dr. David Rogers for encouraging me to pursue $\mathrm{ahD}$ and assisting me in cinching in the belt on this particular body of knowledge. I would like to acknowledge my parents, Diane and Paul Walters, for their love and their support in every venture, of which this $\mathrm{PhD}$ represents one of the more sane. Most of all, I would like to acknowledge my wife, Jill, for her patience when required, impatience when needed, her belief in me and, of course, her love. You are my greatest inspiration. 



\section{List of Publications}

\section{Original articles}

B.R.B. Walters, "Increasing efficiency of BEAMnrc-simulated Co-60 beams using directional source biasing”, Medical Physics 42:5817-5827(2015).

G.S. Duane, Y. Wang, B.R. Walters, J.K. Kim, "A pulse sequence optimization method for assessment of nucleus size in q-space analysis of idealized cells", Journal of Magnetic Resonance 238:115-125(2014).

B.R.B. Walters, R. Kramer, I. Kawrakow, "Dose to medium versus dose to water as an estimator of dose to sensitive skeletal tissue", Physics in Medicine and Biology 55:4535-4546(2010).

B.R.B. Walters, G.X. Ding, R. Kramer, I. Kawrakow, "Skeletal dosimetry in cone beam computed tomography”, Medical Physics 36:2915-2922(2009).

B.R.B. Walters, I. Kawrakow, “A “HOWFARLESS” option to increase efficiency of homogeneous phantom calculations with DOSXYZnrc", Medical Physics 34:3794-3807(2007).

B.R.B. Walters, I. Kawrakow, "Technical note: Overprediction of dose with default PRESTA-I boundary crossing in DOSXYZnrc and BEAMnrc", Medical Physics 34:647-650(2007).

I. Kawrakow, B. R. B. Walters, "Efficient photon beam dose calculations using DOSXYZnrc with BEAMnrc", Medical Physics 33:3046-3056(2006).

D. Sheikh-Bagheri, I. Kawrakow, B. Walters, D. W. O. Rogers, "Monte Carlo simulations: Efficiency improvement techniques and statistical considerations", Integrating New Technologies into the Clinic: Monte Carlo and Image-Guided Radiation Therapy, Proceedings of the 2006 
AAPM Summer School:71-91 (Medical Physics, Madison, WI, 2006).

E. Tonkopi, M. McEwen, B.R.B. Walters, I. Kawrakow, "Influence of ion chamber response on in-air profile measurements in megavoltage photon beams", Medical Physics 32:2918-2927(2005).

I. Kawrakow, D.W.O. Rogers, B.R.B. Walters, "Large efficiency improvements in BEAMnrc using directional bremsstrahlung splitting”, Medical Physics 31:2883-2898(2004).

B.R.B. Walters, I. Kawrakow, D.W.O. Rogers, "History by history statistical estimators in the BEAM code system", Medical Physics 29:2745-2752(2002).

\begin{abstract}
s
B. Walters, I. Kawrakow, "Increasing efficiency of BEAMnrc-simulated Co-60 beams using directional source biasing (DSB)", $5^{\text {th }}$ International Workshop on Monte Carlo Techniques in Medical Physics, June 2014, Quebec City, Canada.
\end{abstract}

B. Walters, G.S. Duane, J.K. Kim, "A Monte Carlo study of the effects of cell membrane permeability on DWI-MRI contrast with oscillating diffusion gradients.", International Society for Magnetic Resonance in Medicine $19^{\text {th }}$ Annual Meeting, May 2011, Montreal, Canada.

B.R.B. Walters, I. Kawrakow, "Increasing efficiency of BEAMnrc-simulated Co-60 beams using directional source biasing", $3^{\text {rd }}$ McGill Workshop on Monte Carlo Techniques in Radiotherapy Delivery and Verification, May 2007, Montreal, Canada

B.R.B. Walters, I. Kawrakow, "Increasing efficiency of DOSXYZnrc calculations”, European Society for Therapeutic Radiology and Oncology (ESTRO) 25, October 2006, Leipzig, Germany.

B. Walters, I. Kawrakow, D. W. O. Rogers, "History by history statistical estimators in the BEAM code system", American Association of Physicists in Medicine (AAPM) $44^{\text {th }}$ Annual Meeting, July 2002, Montreal, Canada.

B. R. B. Walters, I. Kawrakow, D. W. O. Rogers, "Revised Relationship Between R50 and Eo for Electron Beams", World Congress on Medical Physics and Biomedical Engineering, July 2000, Chicago, USA. 


\section{Curriculum Vitae}

Blake Walters was born in Toronto, Ontario, Canada, on November 3, 1966. He attended high school at Richview Collegiate Institute, where he graduated in 1985 summa cum laude. An abiding passion for all things flight related led him to pursue a Bachelor's degree in Aerospace Engineering at the University of Toronto. During his undergraduate education, he sought to broaden the scope of his studies by taking a number of Biology and Biomaterials electives as well as obtaining a summer research position in a Biomaterials lab. After receiving his diploma cum laude in 1989, Blake's growing interest in the application of Physics and Engineering to medicine led him to apply for a summer research position under Dr. Milton Woo at the Toronto Bayview Cancer Clinic (now Sunnybrook Clinic) where he received his first exposure to Medical Physics. There, he used the EGS4 Monte Carlo code to calculate dose deposition kernels for the purpose of estimating dose due to backscattered radiation. Believing radiotherapy to be a somewhat brutal and approximate technique for cancer treatment but maintaining an interest in Medical Physics, Blake applied to the graduate program at the University of Toronto's Department of Medical Biophysics, where he studied magnetic resonance imaging (MRI) under Dr. Michael Bronskill. His Masters thesis dealt with the effects of diffusion of water molecules on the spin-spin (T2) relaxation components of magnetic resonance signal decay, specifically attempting to quantify the effect of cellular dimensions on the various relaxation components. Upon receiving his MSc in 1992, his first full time job took him to the Institute for Biodiagnostics at the National Research Council of Canada (NRC), where he was involved in research on the use of MRI to quantify the post-ischemic effects of stroke. Within a year, however, he had obtained employment with Dr. David Rogers at the NRC's Institute for Ionizing Radiation Standards (IRS) working on the BEAM (now BEAMnrc) code. Initially, he was involved in QA testing and improving the user interface of the code. As his fluency in Monte Carlo techniques grew, however, Blake became increasingly involved in augmenting the functionality of the EGSnrc/BEAMnrc system, itself. It was during the early years working with BEAM that Blake composed the words to the now-canonical BEAM song and unveiled it to the research community at the 1996 BEAM Workshop. He is now a core member of the 
EGSnrc/BEAMnrc development team and has been involved in many major developments in the EGS/BEAM codes, including the transition from EGS4 to the improved physics modeling of EGSnrc, the migration to GUI-based code interfaces, the release of EGS/BEAM as open source software, and the implementation of many efficiency improving techniques. He is also a regular member of the faculty at the popular BEAMnrc/EGSnrc workshops and has found himself teaching Monte Carlo Radiation Physics in such far-flung locations as Sydney, Australia; Trieste, Italy; Mumbai, India; and Madison, Wisconsin. Much of this thesis is based on research conducted during his nearly-two decade tenure at the NRC. 


\section{Valorization}

The socioeconomic value of the research presented in this thesis can best be understood in the context of two broad fields operating within the spheres of health care and public health: the field of Radiotherapy and the field of Radiation Protection. In general, more attention has been given to Radiotherapy because there an urgency to improve the treatment of a disease which is likely to touch us all in some manner, it intersects significantly with other areas of medicine (e.g., radiology, oncology), and, historically, it has consumed more private and public funds. In keeping with this, this Valorization will focus mainly on the applications of the above research to Radiotherapy. However, a section on its implications for the field of Radiation Protection is included at the end.

In order to focus this discussion about the socioeconomic value of the research in this thesis, Figure 1 gives a breakdown of the areas of relevance covered here. The reader is encouraged to refer back to this figure.

\section{Radiotherapy}

According to the World Health Organization (WHO)[2], cancer is second only to heart disease as global cause of death, with the most common cancers being, in order, lung, liver, colorectal, stomach and breast. In 2015, cancer was responsible for 8.8 million deaths worldwide, with the number of new cases expected to rise by approximately $70 \%$ over the next two decades. Moreover, approximately $70 \%$ of cancer deaths occur in low-to-middle-income countries, thus placing the greatest cancer care burden on the societies that can least afford it.

Radiotherapy is one of the three pillars of cancer treatment, which also comprise chemotherapy and surgery. It is estimated that $50 \%$ of newly diagnosed cancer cases require radiotherapy, and that radiotherapy, alone or in conjunction with surgery and/or chemotherapy, is responsible for $40 \%$ of cancer cures[3]. Unfortunately, radiotherapy is a costly treatment modality. In the US, the median cost of a course of radiation therapy can range from USD 8,600 for breast cancer to USD 18,000 for prostate cancer[4]. Add to this the average cost of purchasing 


\begin{tabular}{|l|l|l|l|l|l|}
\hline \multirow{2}{*}{ research in this thesis } & \multicolumn{3}{|c|}{ radiotherapy } & \multirow{2}{*}{ R\&D } & \multirow{2}{*}{ clinical } \\
\cline { 2 - 5 } & \multicolumn{2}{|c|}{ radiation protection } \\
\cline { 2 - 5 } & improving accuracy & understanding RBE & $\begin{array}{c}\text { new techniques \& } \\
\text { technologies }\end{array}$ & beam commissioning & \\
\hline history by history statistics & & & & & \\
\hline $\begin{array}{l}\text { directional bremsstrahlung } \\
\text { splitting }\end{array}$ & & & & & \\
\hline HOWFARLESS & & & & & \\
\hline directional source biasing & & & & & \\
\hline $\begin{array}{l}\text { CBCT skeletal dose } \\
\text { calculations }\end{array}$ & & & & & \\
\hline $\begin{array}{l}D_{m} v s D_{w} \text { as specifier of dose } \\
\text { to sensitive tissue in bone }\end{array}$ & & & & & \\
\hline $\begin{array}{l}\text { Use of Burlin cavity theory to } \\
\text { calculate } D_{m} \text { (Discussion) }\end{array}$ & & & & & \\
\hline
\end{tabular}

Figure 1: Areas of socioeconomic relevance for the research presented in this thesis. Note that, given the importance of valid uncertainty estimates in MC, history by history statistics[1] are inseparable from development and applications of EGSnrc and are, thus, relevant across all areas.

a linear accelerator (approximately USD 2.5 million, factoring in facility costs) and personnel costs, and it can easily be seen that the need outstrips capacity in most low-to-middle-income countries.

\section{EGSnrc}

Monte Carlo (MC) simulations continue to play a large role in the development of modern radiotherapy technology. As the most widely-used general purpose MC code in radiotherapy research, EGSnrc has figured in the development of the state-of-the-art of radiotherapy for techniques ranging from brachytherapy to image-guided radiotherapy (IGRT).

Insofar as much of the research contained herein is integral to EGSnrc, then, it, too, has contributed and continues to contribute to the ongoing development of radiotherapy. For the purposes of this Valorization, it is understood that many of the socioeconomic contributions of the research here are delivered through the vehicle of EGSnrc.

As an aside, however, note that EGSnrc is now public domain software (as of 2016), and, therefore, the algorithms described in this thesis have devolved into this domain, where they are currently being put to use by researchers, engineers and developers across the world developing future applications for Radiotherapy, Radiation Protection, Food Irradiation, and other fields beyond the scope of this work. 


\section{Improving Accuracy}

While improving accuracy would seem to be a self-evident goal for radiotherapy, there is very little quantitative data indicating the degree to which regular systematic errors in practice affect treatment outcome. As mentioned in the Discussion of this thesis, AAPM Task Group 105[5] has stated that errors of 5-7\% may have repercussions for tumour control probability (TCP) and normal tissue complication probability (NTCP). And while this seems like an easily attainable margin of error, one must be aware of the many steps in treatment planning over which inaccuracies may accrue. Thus, it is incumbent on practitioners to improve the accuracy of every step of the treatment planning process.

Since MC is the most accurate method of determining radiotherapy dose, it has been embraced as the method of choice for treatment planning, with the stakeholders in its successful implementation including radiation oncologists, clinical physicists, manufacturers of treatment planning systems (TPSs) and treatment devices and, of course, patients. In addition, MC calculated dose is increasingly used as the quantity of interest in preclinical research platforms, such as small animal irradiators. Considerable resources continue to be devoted to expanding the role of $\mathrm{MC}$ in the clinic, but, as noted in the Discussion, more is required.

Research in this thesis with direct application to improving the accuracy of radiotherapy includes the implementation of history by history statistics in EGSnrc[1] and directional bremsstrahlung splitting (DBS)[6] (see Figure 1). As stated in the Discussion, the former has proven its utility in all applications of EGSnrc and is of direct relevance to assessing the accuracy of a simulation, where robust uncertainty estimates are required. Considering the design and development of more accurate TPSs and techniques, then, it is easy to see that, to the extent that EGSnrc plays a role in their development, so does this method of estimating uncertainty. Directional bremsstrahlung splitting (DBS), meanwhile, has emerged as the de-facto technique for efficient photon and X-ray beam simulations and, thus, plays a key role in such TPS development tasks as: 1) development and testing of MC dose calculation engines, 2) verification of virtual source models, 3) providing phase space data libraries.

No discussion of accuracy would be complete without some mention of the occasional radiotherapy accidents, stemming from human error and/or machine failure, that result in large treatment errors with acute health repercussions. While the WHO estimates that only 3,125 patients worldwide were affected by such incidents between 1976 and 2007[7], the results are alarming and tend to generate press headlines. Although, strictly speaking, such errors fall outside the definition of accuracy as used in this thesis, EGSnrc and other MC simulations are commonly used to simulate these incidents in an attempt to reproduce the levels of overdose/underdose and to help ensure that the errors are not repeated. In this way, then, the 
research in this thesis also comprises a part of the larger effort to insure against such accidents.

\section{Understanding Radiobiological Effect (RBE)}

Given radiotherapy's long history and, in recent decades, relative efficacy, surprisingly little is known about the biological mechanism(s) whereby ionizing radiation destroys tumour cells. Although promising strides have been made in recent years-for example, it is now understood that ionizing effects occur in water-bound DNA and on a scale of nm-this is an area that continues to be of great interest to oncologists, radiobiologists and medical physicists. For it is thought that a more complete understanding of RBE will, in turn, inform the design of more effective radiotherapy techniques.

It is at this intersection of Radiobiology and Radiation Physics that the debate continues over whether dose to water-in-medium, $\mathrm{D}_{w}$, or dose to medium, $\mathrm{D}_{m}$, is a more accurate representation of RBE. The study on whether $\mathrm{D}_{w}$ or $\mathrm{D}_{m}$ is a better estimator of dose to sensitive skeletal tissue [8] presented in this thesis contributes directly to this debate by offering numerical evidence in favour of $\mathrm{D}_{w}$. While the calculation of $\mathrm{D}_{w}$ using Burlin cavity in a mouse phantom, presented in the Discussion, offers a potential general methodology for converting $\mathrm{D}_{m}$ to $\mathrm{D}_{w}$.

In addition, the study on additional dose due to cone beam CT (CBCT) imaging in IGRT[9] presented here contributes, through its deconstruction of skeletal dose into dose to red bone marrow (RBM) and bone surface cells (BSC) to our understanding of RBE.

\section{New Radiotherapy Techniques \& Technologies}

Monte Carlo continues to operate at the forefront of Radiotherapy research, providing a tool that can be used to study new techniques and devices and assisting in the design and QA of new protocols. Thus, MC, and EGSnrc in particular, is of direct interest to physicists and engineers involved in the design and testing of new radiotherapy technologies, which, ultimately, will have an impact on the practices of clinicians and the quality of treatment received by patients.

Several studies in this thesis are relevant to the continuing development of radiotherapy technology. By providing physicists and engineers with a means to efficiently simulate photon beams, directional bremsstrahlung splitting[6] is implicated in any development effort involving high energy photons or X-rays making use of EGSnrc. Directional source biasing[10], meanwhile, with its efficient simulations of Co-60 treatment heads, provides a potential means for commissioning units using the relatively new image-guided radiotherapy (IGRT) technology of MRI combined with Co-60 treatment. Revealing a significant increase in dose to radiosensitive bone surface cells when CBCT is used with IGRT, the dose calculations presented 
in chapter VI[9] provide motivation for improvements in this IGRT technique. Finally, the protocol for using Burlin cavity theory to compute $\mathrm{D}_{w}$ from $\mathrm{D}_{m}$ presented in the Discussion is anticipated to comprise part of a larger body of research that will guide the development of preclinical research tools.

Additionally, the costliness of radiotherapy (see above) has led to a renewed interest in the use of Co-60 treatment heads, especially in low- and middle-income countries, where purchasing the number of LINACs required to meet the treatment requirement is beyond reach. This, then, does not represent the development of a new technology but the re-emergence and refinement of an older technology to meet a current need. The design and commissioning of such units will require efficient MC simulations of Co-60 sources, and in this directional source biasing[10] may play a role.

\section{Beam and TPS Commissioning}

In addition to expanding and refining radiotherapy techniques, MC, and EGSnrc, is used by clinical physicists in the task of commissioning beams and TPSs. Commissioning is an essential process in establishing a new treatment head and/or TPS in a clinic and ensures that: a) the treatment beam delivers the expected dose and $b$ ) the TPS provides an accurate estimate of the delivered dose. Thus, commissioning has a direct impact on the successful operation of a radiotherapy clinic.

For some years, MC calculations have been used for direct comparison with measurement and for providing simulated beam data for use by TPSs. Also, MC-calculated correction factors are used in dose calculation protocols. This indicates the extent of the role that MC plays in radiotherapy practices and also the confidence that clinicians have in current MC algorithms.

Several of the studies in this thesis are relevant to commissioning activities. Once again, DBS[6] is an important technique for any commissioning activity involving photon beams. The HOWFARLESS algorithm for fast DOSXYZnrc dose calculations in homogeneous phantoms[11] is also useful for calculating depth-dose curves and dose profiles for comparison with measurement. Directional source biasing[10] (DSB) has a potential role in commissioning Co-60 units.

Moreover, DSB is of potential relevance to clinics and primary standards labs, such as the National Research Council of Canada, where Co- 60 provides the accepted standard beam quality for ion chamber calibration, because it makes routine comparison between measured and calculated ion chamber doses feasible. 


\section{Radiation Protection}

While cancer treatment and diagnosis represent the most systematically directed application of radiation to the human body, they by no means represent the only sources of radiation exposure. Of course, the most dramatic examples of radiation exposure historically have come from nuclear reactor incidents and the atomic bombs dropped by the US on Hiroshima and Nagasaki at the end of World War II. Though the application of a systematic study of the effects of radiation and accompanying efforts to minimize risk to the public would seem to be quite beside the point in most of these events, useful data on the long-term effects of radiation exposure have been gleaned from these tragedies. These data have allowed researchers working in the field of Radiation Protection to estimate the risks associated with unintended exposure to various sources of radiation, including naturally occuring terrestrial and cosmic sources, as well as occupational risks for those who work with or close to radiation (e.g., nuclear power workers, health care workers).

The International Commission on Radiological Protection (ICRP) estimates that the additional risk of cancer death due to exposure to radiation is 5.5\% per Sievert (Sv)[12], where a Sievert is the SI unit for dose equivalent and is equal to aborbed dose scaled by a quality factor, depending on the type of radiation, and the radiobiological effect. The exposure limits currently proposed by the ICRP[12] are $1 \mathrm{mSv} / \mathrm{yr}$ for the general public and $20 \mathrm{mSv} / \mathrm{yr}$ for radiation workers. Note that these limits apply to intentional or accidental exposure and, thus, are over and above unavoidable exposure to radiation occuring naturally in the environment. Governments of some countries, including Canada, use these same exposure limits. While it is a somewhat tired adage to equate the lifetime risk represented by exposure to $1 \mathrm{mSv} / \mathrm{yr}$ to one-fifth that of smoking a lifetime total of 75 cigarettes[13], it nevertheless puts the risks represented by these exposure limits into perspective. Quite apart from any scientific/actuarial basis for these limits, however, most public policy is informed by the perception of radiation as generally dangerous, and so these represent absolute upper limits for guidelines adhering to the practice of keeping exposure as low as reasonably allowable, or the so-called ALARA principle.

Given the uneasy balance between perceived and actual risk, then, it is important from both public safety and policy perspectives that: 1) the public be shielded from unintended exposure to human-manufactured sources of radiation as much as possible and 2) policy be guided by an understanding of potential sources of exposure and radiobiological effects.

While the design of radiation shielding structures, such as LINAC bunkers, generally does not require the accuracy available in MC simulations, there is, nevertheless, an ongoing role for EGSnrc in determing the dose distribution around an irradiating device and, hence, the 
material and layout of the shielding strucure.

The research in this thesis, however, is more pertinent to the goal of understanding radiobiological effects and mitigating exposure where possible. In quantifying additional dose to red bone marrow and bone surface cells, the dosimetric study of the routine use of CBCT imaging in IGRT[9] motivates guidelines for the use of this particular imaging modality. The study on $\mathrm{D}_{m}$ versus $\mathrm{D}_{w}$ as an accurate representation of dose to sensitive tissue in bone[8], meanwhile, presents a quantitative argument for $\mathrm{D}_{w}$ as a better estimator of RBE and, consequently, risk in the case of unintended or avoidable exposure. By this same token, the research on estimating $\mathrm{D}_{w}$ presented in the Discussion may also be directed towards a method for estimating risk from radiation exposure.

\section{Other Applications}

Although EGSnrc has been developed with therapeutic, diagnostic and radiation protection applications in mind, the above discussion by no means represents the full extent of its societal role, and, by extension, the role of much of the research in this thesis. For example, companies designing LINACs for industrial purposes, such as food irradiation and equipment sterilization, make use of EGSnrc simulations to optimize accelerator design, determine shielding requirements and maximize throughput.

It is expected that, as the capabilities of EGSnrc evolve and expand in response to interest in new applications of ionizing radiation and new treatment modalities (see the Discussion), the research described in this thesis will find other applications of socioeconomic consequence heretofore unseen.

\section{Bibliography}

[1] B. R. B. Walters, I. Kawrakow, and D. W. O. Rogers. History by history statistical estimators in the BEAM code system. Technical Report PIRS-0791, National Research Council of Canada, Ottawa, Canada, 2002.

[2] World Health Organization. Cancer Fact Sheet. http://www.who.int/mediacentre/factsheets/fs297/en/, 2017.

[3] GlobalRT. About Radiotherapy. http://globalrt.org/about-radiotherapy/, 2017.

[4] A.J. Paravati et al. Variation in the Cost of Radiation Therapy Among Medicare Patients With Cancer. J. Oncology Practice, 11:403-409, 2015. 
[5] Indrin Chetty et al. Guidence report on clinical implementation of the Monte Carlo method in external beam radiation therapy treatment planning: Report of the AAPM Task Group No. 105. Med. Phys., page submitted, 2005.

[6] Iwan Kawrakow, D. W. O Rogers, and B.R.B. Walters. Large efficiency improvements in BEAMnrc using directional bremsstrahlung splitting. Med. Phys., 31:2883 - 2898, 2004.

[7] World Health Organization. Radiotherapy Risk Profile: Technical Manual. 2008.

[8] B. R. B. Walters, R. Kramer, and I. Kawrakow. Dose to medium versus dose to water as an estimator of dose to sensitive skeletal tissue. Phys. Med. Biol., 55:4535 - 4546, 2010.

[9] B. R. B. Walters, G. X. Ding, R. Kramer, and I. Kawrakow. Skeletal dosimetry in cone beam computed tomography. Med. Phys., 36:2915 - 2922, 2009.

[10] B. R. B. Walters. Increasing efficiency of BEAMnrc-simulated Co-60 beams using directional source biasing. Med. Phys., 42:5817 - 5827, 2015.

[11] B. R. B. Walters and I. Kawrakow. A "HOWFARLESS" option to increase efficiency of homogeneous phantom calculations with DOSXYZnrc. Med. Phys., 34:3794 - 3807, 2007.

[12] ICRP. The 2007 Recommendations of the International Commission on Radiological Protection. Annals of the ICRP 103, ICRP, Washington D.C., 2007.

[13] H. E. Johns and J. R. Cunningham. The Physics of Radiology, Fourth Edition. Charles C. Thomas, Springfield, Illinois, 1983. 
